\title{
Designing New Atomic Layer Deposition Precursors for Third-Generation Photovoltaics
}

\author{
by
}

\section{Goran Bačić}

A thesis submitted to the Faculty of Graduate and Postdoctoral Affairs in partial fulfillment of the requirements for the degree of

\author{
Doctor of Philosophy \\ in \\ Chemistry \\ Carleton University
Ottawa, Ontario
}

(C) 2020

Goran Bačić 
This thesis is dedicated to my parents, Eugen and Jagoda 


\section{Abstract}

Atomic layer deposition (ALD) holds a critical position in nanofabrication because of its excellent uniformity, conformality to nanostructured surfaces, subnanometer thickness control, and ability to synthesize high-quality materials and interfaces. Its abilities arise from its chemical mechanism: precursor molecules saturate the surface with a selflimiting monolayer and prevent continuous growth of material, then react with another co-reactant to deposit about one atomic layer of material at a time. Consequently, ALD first requires precursors with suitable volatility, chemical reactivity, and thermal stability. Motivated by the prospects of using ALD to fabricate next-generation solar cells and the unusual quantum mechanical behaviour of $\mathrm{Pb}$-containing materials at the nanoscale, we set out to design, develop, and understand new $\mathrm{Pb}$-containing ALD precursors. We began by attempting to prepare $\mathrm{Pb}(\mathrm{II})$ analogues of $N$-heterocyclic carbenes, eventually finding the stable lead(II) rac- $N, N^{\prime}$-di-tert-butylbutane-2,3-diamide (1Pb) that displayed the best volatility of any $\mathrm{Pb}(\mathrm{II})$ complex reported in the literature (1 Torr vapour pressure at $\left.94^{\circ} \mathrm{C}\right)$. With $\mathbf{1 P b}$ and the well-known lead(II) bis[bis(trimethylsilylamide)] (0Pb), we then developed an ultralow-temperature ALD process for $\mathrm{PbS}$ using $\mathrm{H}_{2} \mathrm{~S}$ as the S-containing co-reactant. Pure and crystalline PbS films were deposited with ideal ALD growth behaviour at temperatures as low as $45^{\circ} \mathrm{C}$ with $\mathbf{1 P b}$ and $65^{\circ} \mathrm{C}$ with $\mathbf{0 P b}$, enabling deposition on thermally sensitive substrates like $\mathrm{CH}_{3} \mathrm{NH}_{3} \mathrm{PbI}_{3}$ (MAPI) perovskites. Not only did the process effectively encapsulate MAPI thin films, they also had tunable $p$-type conductivity, hole mobility, and carrier concentrations. An unusual reduction to elemental $\mathrm{Pb}(0)$ of $\mathbf{1 P b}$ by $\mathrm{H}_{2} \mathrm{~S}$ was found to begin at $95^{\circ} \mathrm{C}$ and was studied experimentally and theoretically by density functional theory (DFT) to understand its mechanism. Despite their success at low temperatures, $\mathbf{0 P b}$ and $\mathbf{1} \mathbf{P b}$ were limited by their thermal stability $\left(<155^{\circ} \mathrm{C}\right)$, so we set out to design a more stable $\mathrm{Pb}$-containing precursor. The surprising volatility of the dimeric bis[lead(II) N,N'-di-tert-butyl-1,1-dimethylsilanediamide] [(4Pb $\left.)_{2}\right]$ prompted an in-depth theoretical study of its bonding, thermodynamics, and molecular electrostatic properties. By studying $(\mathbf{4 P b})_{2}$ and some of its derivatives, we gained a more nuanced view of the chemical nature of volatility with implications for future precursor design. The success of $(\mathbf{4 P b})_{2}$ led us to adapt its ligand for Co, yielding the unprecedented spirocyclic low-spin $\mathrm{Co}(\mathrm{IV})$ bis[ $N, N^{\prime}$-di-tert-butyl-1,1-dimethyldiamidosilane] (4Co). Finally, we explored $\mathrm{Sn}(\mathrm{II})$ and $\mathrm{Sn}(\mathrm{IV})$ trifluoroacetates as green potential ALD precursors to the transparent conductor F-doped $\mathrm{SnO}_{x}$. We analyzed their structure and bonding by X-ray diffraction (XRD) in the solid state, and gas-phase electron diffraction (GED) and DFT in the vapour phase, showing that their polymeric structures actually increased their volatility, and may represent a new precursor design strategy altogether. 


\section{Preface}

This preface provides full bibliographical details for each article included in this thesis, as well as whether the article is reproduced in whole or in part. Use of copyrighted material is likewise acknowledged here. When citing material from this thesis, please cite the article relevant to the chapter if the chapter is based on a publication. All published Supplementary Information is freely available via the Internet. Please direct any request for unpublished Supplementary Information to gibacic@gmail.com.

Pursuant to the Integrated Thesis policy of Carleton University, the supervisor (Seán T. Barry) and the "student" (Goran Bačić) confirm that the student was fully involved in setting up and conducting the research, obtaining data and analyzing results, as well as preparing and writing the material presented in the co-authored article(s) integrated in the thesis. Additionally, the supervisor confirms the information provided by the student in this preface.

\section{Chapter 2}

Goran Bačić, David Zanders, Bert Mallick, Anjana Devi, and Seán T. Barry. Designing Stability into Thermally Reactive Plumbylenes. Inorg. Chem, 2018, 57, 14, 8218-8226. Copyright 2018 American Chemical Society.

This publication is wholly reproduced and edited for formatting and clarity of presentation. Goran Bačić designed the experiments. Synthesis was carried out equally by Goran Bačić and David Zanders. X-ray crystallography was performed by Bert Mallick. Additional DFT calculations were performed by Goran Bačić. The publication was written by Goran Bačić.

\section{Chapter 3}

Georgi Popov, Goran Bačić, Miika Mattinen, Toni Manner, Hannu Lindström, Heli Seppänen, Sami Suihkonen, Marko Vehkamäki, Marianna Kemell, Pasi Jalkanen, Kenichiro Mizohata, Jyrki Räisänen, Markku Leskelä, Hanna Maarit Koivula, Seán T. Barry, and Mikko Ritala. Atomic Layer Deposition of PbS Thin Films at Low Temperatures. Chem. Mater., 2020, 32, 19, 8216-8228. Copyright 2020 American Chemical Society.

This publication is wholly reproduced and edited for formatting and clarity of presentation. Georgi Popov performed the experiments for this publication. The article was written by Georgi Popov with precursor synthesis and theoretical calculations performed and discussed by Goran Bačić. 


\section{Chapter 4}

Goran Bačić, Jason D. Masuda, and Seán T. Barry. Designing Volatility into Thermally Stable Plumbylenes, In Preparation, 2020.

This manuscript is wholly reproduced and edited for formatting and clarity of presentation. Goran Bačić designed and performed the experiments and theoretical calculations. X-ray crystallography was performed by Jason D. Masuda. The manuscript was written by Goran Bačić.

\section{Chapter 5}

David Zanders, Goran Bačić, Dominique Leckie, Oluwadamilola Odegbesan, Jeremy Rawson, Jason D. Masuda, Anjana Devi, and Seán T. Barry. A Rare Low-Spin Co ${ }^{\text {IV }} \operatorname{Bis}(\beta-$ silyldiamide) with High Thermal Stability: Steric Enforcement of a Doublet Configuration. Angew. Chem. Int. Ed., 2020, 59, 14138-14142.

This publication is wholly reproduced and edited for formatting and clarity of presentation. David Zanders designed and performed synthesis and thermal characterization. Goran Bačić performed all theoretical calculations and analysis for this work. Dominique Leckie and Jeremy Rawson performed electron paramagnetic resonance (EPR) spectroscopy. Oluwadamilola Ogedbesan performed initial synthetic experiments. X-ray crystallography was performed by Jason D. Masuda. The article was written equally by David Zanders and Goran Bačić.

\section{Chapter 6}

Goran Bačić, Conor D. Rankine, Jason D. Masuda, Derek A. Wann, and Seán T. Barry. Volatile and Thermally Stable Polymeric Tin Trifluoroacetates, Inorg. Chem., 2020, 59, 2, 996-1005. Copyright 2020 American Chemical Society.

This publication is wholly reproduced and edited for formatting and clarity of presentation. Goran Bačić designed the experiments and performed synthesis, spectroscopy, and thermal characterization. Gas-phase electron diffraction (GED) and initial density functional theory (DFT) calculations were performed by Conor D. Rankine. X-ray crystallography was performed by Jason D. Masuda. Additional DFT calculations were performed by Goran Bačić. The publication was written by Goran Bačić. 


\section{Acknowledgements}

I would first and foremost like to thank my supervisor, Prof. Seán T. Barry, for accepting the risk of hiring a graduate student with no synthetic experience and minor delusions of grandeur all those years ago. Without your encouragement, experience, collaborative spirit, support, and relentless search for funding, none of the work presented in this Thesis would have been possible. Not only did you supervise my development as a scientist, but your friendship helped me get through the usual and unusual obstacles I encountered during my studies. The warm and welcoming research environment that is the Barry Lab over the last 5 years let me grow into the person I am today.

Of course, much of this work would not have been possible without my collaborators across Canada, and overseas in Germany and England. Just as crucial, I want to thank the staff at Carleton University - Dr. Daniel Sun, Chantelle Gravelle, Peter Mosher, Jim Logan, Susa Chandrabose, Tanya Rudd, Kyle Fournier, Prof. Bob Burk, Rob Vandusen, and Angela Williams - who made my work and day-to-day life that much better with your help, kind words, and great conversation. Special thanks to Dr. Sharon Curtis for ecstatically performing electron-impact mass spectrometry at the University of Ottawa on my crazy samples; Prof. Toby Zeng for throwing me in the deep end of quantum chemistry and fostering a deep appreciation of the field; Prof. Erin Johnson at Dalhousie University for the patient hand-holding when I was painfully new to DFT; and a huge thanks to Prof. Steve McGarry, who has become a close friend and advisor over the years. I deeply appreciate the exciting science I was able to produce with you, but I appreciate you even more.

My group members, past and present, deserve special thanks. Dr. Peter Gordon, for your jokes, advice, collaboration, music, patience, and gentle reminders that I ought to relax just a little. David Zanders, for your boundless optimism, sunny disposition, tireless persistence, and extreme scientific rigour; all of which culminated in two beautiful papers. Mike Land, for the laughs, a taste of the East Coast, watching my back, and not to mention the endless knowledge on the chemical literature that helped me so much. Eden Goodwin, for the always fresh perspective, friendship, dank memes, and humour (I hardly know her). Zack Dubrawski, for being the friend that was always there to encourage me, show me around at conference mixers, and interrupt my playlists with gorgeous Baroque performances. Joey Zurakowski, for being the yin to my yang, having an inspiring latent ability in chemistry, and just being the chillest. Erica Dionisi, our honorary group member, for sharing your food, being so thoughtful, kind, funny, and always having something nice to say about everyone. My undergraduate students, Dami Odegbesan and Ian Frankel, for taking my half-baked projects and going all in on them; you two taught me more than I taught you. Drs. Peter Pallister, Dave Mandia, and Jason Coyle, the previous giants of the lab, for showing me that my studies can be fun and rewarding, staying in touch over all these years and all these miles, and providing a great example for my post-graduate life. Dr. Sydney Buttera, for always being there when I needed someone to talk to, laughing at my jokes, and being in the club before 11 o'clock; I'm lucky to have a friend and colleague as kind and loving as you. Last, but definitely not least, Dr. Matthew Griffiths, for teaching me to love chemistry, sharing your delicious cooking, being a close confidant, keeping me down to earth, and (literally) living with me over the years. 
My close friends that stuck by my side during the last five years deserve individual recognition. Ron Deschamps, Owen Clayton, James Podrebarac, Aaron Coutino, Josh Scammel, Raf Pineda, Adam Cichoński, Scott Mittelstädt, Jenna Robertson, Martin Szklarczyk, and Damjan Marković. Here's to many more years!

Most of all, I would like to thank my family. To my parents Eugen and Jagoda Bačić, who raised me with unconditional and unending love. To my big sister Ariana, brother-in-law Shawn, and my little niece Olivia VanSnick, for letting me live with you rent-free (!) and giving me more love than I can even handle. To Babi Mira, Dida Ive, and Babi Dragica, for raising my wonderful parents, enduring the hardships of being fresh immigrants from Hrvatska, sacrificing so we could lead the best lives we could, and always being there while I grew up all these years. And to Christine Camus-Shepley and Bruce Shepley, for welcoming me into their family like one of their own.

Finally, to my partner Kerianne, whom I love with all my heart. I have never met someone who can find every beautiful part of Nature, big or small, like you do. You took care of me during the hardest year of my life, and I never would have completed the last year of my Doctoral studies during COVID-19 if it weren't for you. Your support, skills, critique, humour, love, and grace changed me profoundly and for the better. Not to mention that this thesis would not be nearly as pretty if I didn't defer to you on colour. I love you. 


\section{Contents}

Preface iv

$\begin{array}{ll}\text { Acknowledgements } & \text { vi }\end{array}$

$\begin{array}{ll}\text { Contents } & \mathbf{x} \\ & \end{array}$

1 Introduction $\quad 1$

1.1 Third-Generation Photovoltaics (3GPVs) $\ldots \ldots \ldots \ldots \ldots \ldots$

1.1.1 Motivation . . . . . . . . . . . . . . . . . 1

1.1.2 Target Materials . . . . . . . . . . . . . . . . . 2

1.1.2.1 Transparent-Conducting Materials: F-doped $\mathrm{SnO}_{x} \ldots \ldots \ldots 2$

1.1.2.2 Light-Absorbing Materials: Pb-based Semiconductors . . . . . . 3

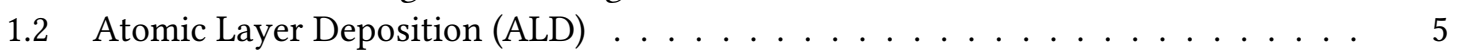

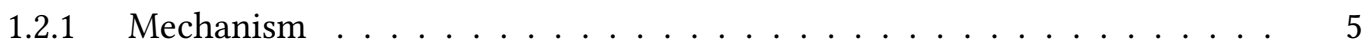

1.2.2 Growth Behaviour \& Thin Film Quality . . . . . . . . . . . . 6

1.2.2.1 Subnanometer Thickness Control . . . . . . . . . . . . 6

1.2.2.2 Uniformity \& Conformality . . . . . . . . . . . . 7

1.2.2.3 Interface \& Composition Control . . . . . . . . . . . 7

$1.2 .3 \quad$ ALD \& $3 \mathrm{GPV} \ldots \ldots \ldots \ldots \ldots \ldots$

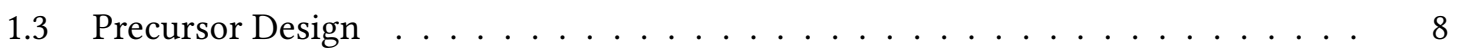

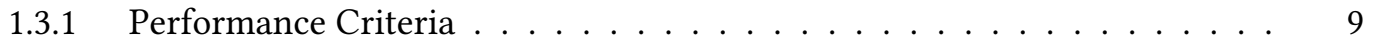

1.3.1.1 Volatility .................... 9

1.3.1.2 Thermal Stability . . . . . . . . . . . . . . . . . 11

1.3.1.3 Chemical Reactivity . . . . . . . . . . . . . . . . . . . . 11

1.3.2 Thermogravimetric Analysis (TGA) . . . . . . . . . . . . . . . 12

2 Designing Stability Into Thermally Reactive Plumbylenes $\quad 15$

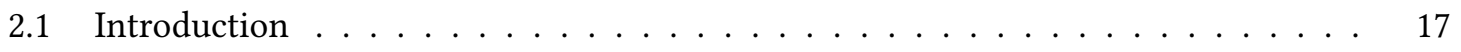

2.2 Results and Discussion . . . . . . . . . . . . . . . . . . . . 19

2.2.1 $\quad N^{2} N^{3}$-di-tert-butylethylene-2,3-diamido lead(II) (3Pb) $\ldots \ldots \ldots \ldots$

2.2.2 $\quad N^{2} N^{3}$-di-tert-butylethane-2,3-diamido lead(II) (2Pb) . . . . . . . . . . 20

2.2.3 Rac- $N^{2} N^{3}$-di-tert-butylbutane-2,3-diamide lead(II) (1Pb) . . . . . . . . 23

2.2.3.1 Solid-state and solution-phase structure . . . . . . . . . 25

2.2.3.2 Volatility and thermal stability of $\mathbf{1 P b} \ldots \ldots \ldots 28$

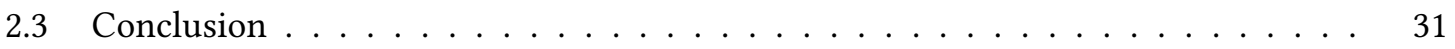

2.4 Experimental Details . . . . . . . . . . . . . . . . . . . . 31

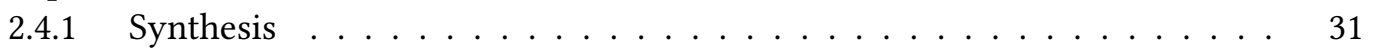

2.4.1.1 General Considerations . . . . . . . . . . . . . . . . 31 
2.4.1.2 N,N'-di-tert-ethylene-2,3-diimine (DAD) $\ldots \ldots \ldots \ldots . \ldots . \ldots 32$

2.4.1.3 Rac-N,N'-di-tert-butylbutane-2,3-diamine . . . . . . . . . . 32

2.4.1.4 Bis[bis(trimethylsilyl)amido] lead(II) $(\mathbf{0 P b}) \ldots \ldots 33$

2.4.1.5 Attempted synthesis of rac- $^{2}, \mathrm{~N}^{2}$-di-tert-butylbutane-2,3-diamido lead(II) $(\mathbf{1 P b})$ via salt metathesis of $\mathrm{PbCl}_{2} \ldots \ldots \ldots . . . . .34$

2.4.1.6 Successful preparation of $\mathbf{1 P b}$ via transamination . . . . . . 35

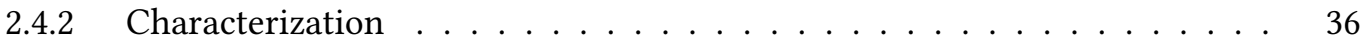

2.4.2.1 In-situ NMR spectroscopy of the attempted preparation of $\mathbf{2} \mathbf{P b} 36$

2.4.2.2 Thermogravimetric analysis (TGA) . . . . . . . . . . 36

2.4.2.3 In-situ NMR spectroscopy of thermolysis of $\mathbf{1 P b} \ldots \ldots \ldots 37$

2.4.2.4 Neat thermolysis of $\mathbf{1} \mathbf{P b} \ldots \ldots \ldots . \ldots 37$

2.4.2.5 Single-crystal X-ray diffraction of $\mathbf{1} \mathbf{P b} \ldots \ldots \ldots 38$

2.4.2.6 Powder X-ray diffraction . . . . . . . . . . . . . . 38

3 Ultralow Temperature Atomic Layer Deposition of PbS 40

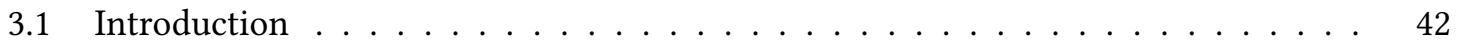

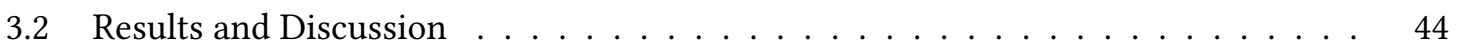

3.2.1 Precursor Characteristics, Synthesis, and Scale-Up . . . . . . . . . . . . . 44

3.2 .2 PbS Film Growth . . . . . . . . . . . . . . . . . . . . . . . 45

3.2.3 Film Composition and Crystallinity . . . . . . . . . . . . . . . . . . 47

3.2.4 Formation of $\mathrm{Pb}$ Using $\mathbf{1 P b} \ldots \ldots \ldots \ldots$. . . . . . . . . . 48

3.2 .5 Film Morphology . . . . . . . . . . . . . . . . . . . . 53

$3.2 .6 \quad$ Electrical Properties . . . . . . . . . . . . . . . . . . . 56

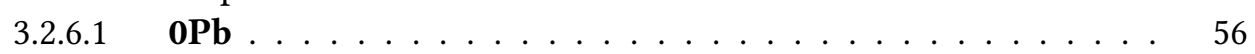

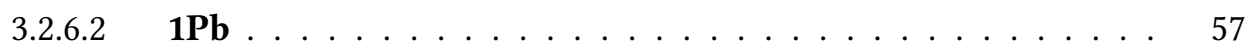

$3.2 .7 \quad$ Optical Properties . . . . . . . . . . . . . . . . . . 58

3.2.8 Deposition of PbS onto Halide Perovskites . . . . . . . . . . . . . . . . . 64

3.2.9 Application of $\mathrm{PbS}$ as a Protective Layer . . . . . . . . . . . . . . 67

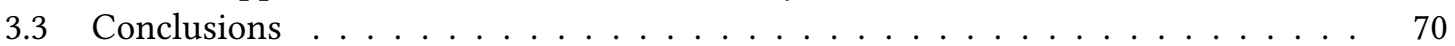

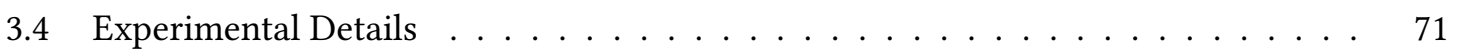

$3.4 .1 \quad$ Precursor Synthesis . . . . . . . . . . . . . . . . . . . . . 71

3.4 .2 Film Deposition . . . . . . . . . . . . . . . . . . 72

3.4.3 Film Characterization . . . . . . . . . . . . . . . . . 74

3.4.4 Computational Details . . . . . . . . . . . . . . . . . 76

4 Designing Volatility Into Thermally Stable Plumbylenes $\quad 77$

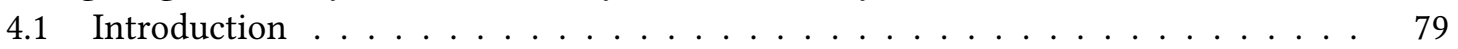

4.2 Results and Discussion . . . . . . . . . . . . . . . . . . 80

4.2.1 Lead(II) $N, N^{\prime}$-di-tert-butyl-1,1-dimethylsilanediamide . . . . . . . . . . . . . . 80

4.2 .2 Increasing Steric Bulk . . . . . . . . . . . . . . . . . . . 85

4.2 .3 Ancillary Ligands . . . . . . . . . . . . . . . . . . . . . . . . 88

4.2 .4 Pendant Tertiary Amines . . . . . . . . . . . . . . . . . . . 92

4.2.5 Towards a Speculative Precursor Design Process _ . . . . . . . . . . . . 96

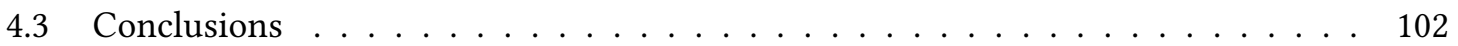

4.4 Experimental Details . . . . . . . . . . . . . . . . . . . . 103

4.4 .1 Synthesis . . . . . . . . . . . . . . . . . . . . 103

4.4.1.1 General Considerations . . . . . . . . . . . . . . . 103

$4.4 .1 .2 \quad(4 \mathbf{P b})_{2} \ldots \ldots \ldots \ldots \ldots \ldots$ 
4.4.1.3 $\mathrm{Me}_{2} \mathrm{Si}\left(\mathrm{NH}^{t} \mathrm{Pe}\right)_{2} \ldots \ldots \ldots \ldots \ldots$

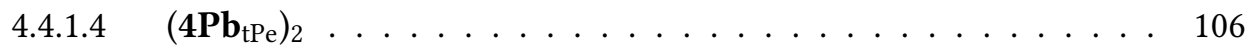

$4.4 .1 .5 \quad(4 \mathrm{~Pb})(\mathrm{PPrNHC}) \ldots \ldots \ldots \ldots \ldots$

4.4.1.6 Lead(II) $N$, $N^{\prime}$-bis(1-(dimethylamino)-2-methylpropan-2-yl)-1,1-

dimethylsilanediamide $(\mathbf{X}) \ldots \ldots \ldots$. . . . . . . 108

4.4 .2 Thermogravimetric Analysis . . . . . . . . . . . . . . . . . . 110

4.4 .3 Computational Details . . . . . . . . . . . . . . . . . . . . . 112

5 A Volatile and Thermally Stable Co(IV) Bis( $\beta$-silyldiamide) 114

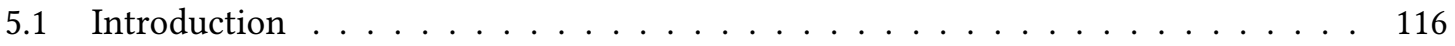

5.2 Results and Discussion . . . . . . . . . . . . . . . . . . 117

5.2.1 Co(IV) Bis[bis(tert-butylamido)dimethylsilane $] \ldots \ldots \ldots$. . . . . . . . 117

5.2.2 Steric Control of the Electronic Structure . . . . . . . . . . . . . . . . . . . 119

5.2.3 Evaporation Kinetics and Self-Limiting Behaviour . . . . . . . . . . . . . . 124

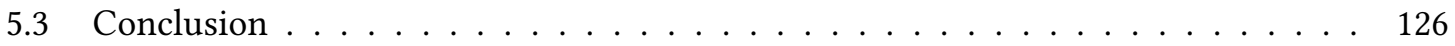

5.4 Experimental Details . . . . . . . . . . . . . . . . . . . . . . 127

5.4.1 General Considerations . . . . . . . . . . . . . . . . . . . . . 127

5.4.2 Synthesis of $\mathrm{Co}\left[\left(\mathrm{N}^{t} \mathrm{Bu}\right)_{2} \mathrm{SiMe}_{2}\right]_{2}(4 \mathrm{Co}) \ldots \ldots \ldots \ldots \ldots \ldots$

5.4.3 Single-Crystal X-Ray Diffraction (XRD) . . . . . . . . . . . . . . . . . . . . 129

5.4 .4 Thermogravimetric Analysis (TGA) . . . . . . . . . . . . . . . . . . . . 130

5.4.5 Differential Scanning Calorimetry (DSC) . . . . . . . . . . . . . . . . . . 130

5.4 .6 Figure of Merit $\sigma \ldots \ldots \ldots \ldots \ldots \ldots \ldots$

5.4.7 Quartz Crystal Microbalance (QCM) Saturation Experiments . . . . . . . 131

5.4 .8 Computational Details . . . . . . . . . . . . . . . . . . . . . 132

6 Volatile and Thermally Stable Polymeric Tin Trifluoroacetates 136

6.1 Introduction . . . . . . . . . . . . . . . . . . . . . . . . 138

6.2 Results and Discussion . . . . . . . . . . . . . . . . . . . . . 139

6.2 .1 Tin(II) Trifluoroacetates . . . . . . . . . . . . . . . . . . . . . . . . 139

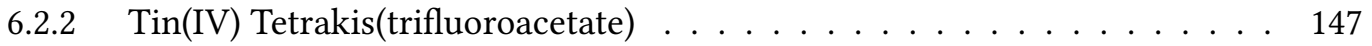

6.2.3 Evaporation Kinetics and Self-Limiting Behaviour . . . . . . . . . . . . . . 148

6.2.4 Supramolecular Organization . . . . . . . . . . . . . . . . . . . 151

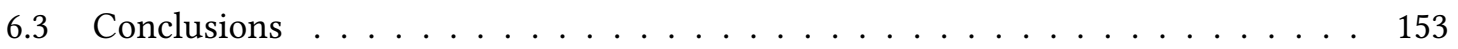

6.4 Experimental Details . . . . . . . . . . . . . . . . . . . . 153

6.4 .1 Synthetic Details . . . . . . . . . . . . . . . . . . . . . 153

6.4.1.1 General Synthetic Considerations . . . . . . . . . . . . . 153

6.4.1.2 Hexatin(II) di- $\mu_{3}$-oxy-octakis- $\mu$-trifluoroacetate (3) . . . . . . 154

6.4.1.3 Tin(IV) tetrakis(trifluoroacetate) (5) . . . . . . . . . . . . 157

6.4 Thermogravimetric Analysis (TGA) . . . . . . . . . . . . . . . . 158

6.4.3 Atomic Layer Deposition Potential of 3 . . . . . . . . . . . . . . . . . . . . . . 159

6.4 .4 X-ray Crystallography . . . . . . . . . . . . . . . . . . . . 160

6.4.5 Theoretical Calculations . . . . . . . . . . . . . . . . . . . 161

6.4.6 Gas-phase Electron Diffraction (GED) . . . . . . . . . . . . . . . . . . 162

6.4.7 Reduction and Refinement . . . . . . . . . . . . . . . . . . . 163

7 Conclusions \& Future Directions $\quad 164$

$\begin{array}{ll}\text { References } & 167\end{array}$ 


\section{List of Figures}

1.1 Quantum confinement in $\mathrm{PbS}$ nanocrystals. . . . . . . . . . . . . . . 4

1.2 Diagram of a single atomic layer deposition (ALD) cycle. . . . . . . . . . . 5

1.3 Equivalent slab volume of ultrathin absorbers on nanowire arrays. . . . . . . . . 8

1.4 Thermogravimetric analysis (TGA) showing a stress-test. . . . . . . . . . . . . . 12

2.1 Notation for five-membered $N$-heterocyclic metallylenes. . . . . . . . . . . . . 17

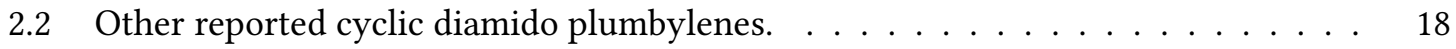

2.3 Failed syntheses of $\mathbf{3 P \mathbf { P }} . \quad \ldots \ldots \ldots \ldots \ldots \ldots$

2.4 Unsaturated NHEs $3 E$ are $6 \pi$-aromatic rings. . . . . . . . . . . . . . . . . 20

2.5 Attempted preparation of $\mathbf{2} \mathbf{P b}$ by transamination. . . . . . . . . . . . . . 22

2.6 In-situ ${ }^{1} \mathrm{H}$ NMR of attempted preparation of $\mathbf{2} \mathbf{P b}$ by transamination. . . . . . . . 23

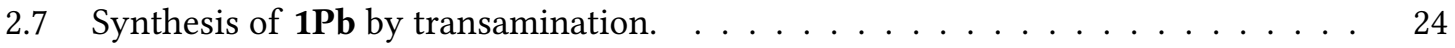

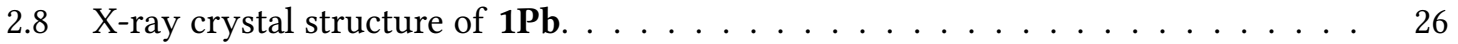

2.9 Weak intermolecular interactions of $\mathbf{1 P b}$ in the solid state . . . . . . . . . . 27

2.10 TGA and vapour pressure of $\mathbf{1 P b}$. . . . . . . . . . . . . . . . . 29

2.11 Proposed $[2+2+1]$ cycloreversion thermolysis of $\mathbf{1 P b}$ at $150{ }^{\circ} \mathrm{C} \ldots \ldots \ldots$

2.12 Experimental (top, black) and simulated (bottom, red) powder X-ray diffraction (pXRD) patterns for $\mathbf{1 P b} \ldots \ldots \ldots \ldots \ldots$

3.1 ALD growth behaviour of $\mathbf{0 P b}$ and $\mathbf{1} \mathbf{P b}$ with $\mathrm{H}_{2} \mathrm{~S} \ldots \ldots \ldots \ldots \ldots$

3.2 GPC of PbS as function of the purge duration and thickness with respect to cycles

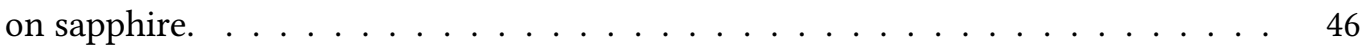

3.3 Uniformity of $\mathrm{PbS}$ ALD compared to TMA- $\mathrm{H}_{2} \mathrm{O}$ ALD. . . . . . . . . . . . . . 47

3.4 Purity (TOF-ERDA) and crystallinity (GIXRD) of PbS ALD thin films. . . . . . . . 48

3.5 Growth of $\mathrm{Pb}(0)+\mathrm{PbS}$ by reduction of $\mathbf{1 P b}$ with $\mathrm{H}_{2} \mathrm{~S}$ at $1355^{\circ} \mathrm{C} \ldots \ldots \ldots$

3.6 GIXRD, photographs, resistivity, and $\mathrm{SEM}$ of $\mathrm{Pb}(0)+\mathrm{PbS}$ by reduction of $\mathbf{1 P b}$ with

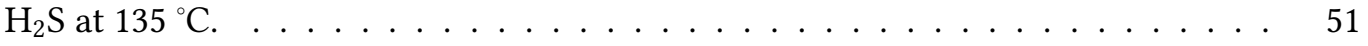

3.7 Spontaneous reduction of $\mathbf{1} \mathbf{P b}$ to $\mathrm{Pb}(0)$ by $\mathrm{H}_{2} \mathrm{~S} \ldots \ldots \ldots \ldots \ldots \ldots \ldots$

3.8 FESEM images of $\mathrm{PbS}$ films deposited at different temperatures using $\mathbf{0 P b}-\mathrm{H}_{2} \mathrm{~S}$. . $\quad 53$

3.9 FESEM images of $\mathrm{PbS}$ films deposited at different temperatures using $\mathbf{1} \mathbf{P b}-\mathrm{H}_{2} \mathrm{~S}$. . $\quad 54$

3.10 FESEM images of $\mathrm{PbS}$ films deposited with different number of cycles using $\mathbf{0} \mathbf{P b}-\mathrm{H}_{2} \mathrm{~S} . \quad 55$

3.11 Electrical characterization of $\mathrm{PbS}$ films. . . . . . . . . . . . . . . 56

3.12 Transmittance of PbS on sapphire deposited by $\mathbf{0 P b}-\mathrm{H}_{2} \mathrm{~S}$, TEM of a PbS film, crystallite size by GIXRD, and electronic transition energies. . . . . . . . . . . . . 58

3.13 Transmittance of $\mathrm{PbS}$ on sapphire deposited by $\mathbf{0} \mathbf{P b}-\mathrm{H}_{2} \mathrm{~S}$ and $\mathbf{1} \mathbf{P b}-\mathrm{H}_{2} \mathrm{~S}$, optical bandgaps as a function of temperature from Tauc plots. . . . . . . . . . . . . 59

3.14 Contour profile maps of out of plane and in-plane XRD data for PbS films deposited with $\mathbf{0 P b} . \ldots \ldots \ldots$. . . . . . . . . . . . . . . . . . . . . . . . 60 
$3.15 \log (1 / T)$ plots of $\mathrm{PbS}$ films deposited on sapphire with $\mathbf{0 P b}$ and $\mathbf{1 P b} . \ldots \ldots \ldots$

3.16 Cross-section SEM of ALD PbS on MAPI. Comparison of $\mathbf{0 P b}-\mathrm{H}_{2} \mathrm{~S}$ and $\mathbf{1} \mathbf{P b}-\mathrm{H}_{2} \mathrm{~S}$. Encapsulation efficiency. And proposed mechanism of MAPI decomposition above

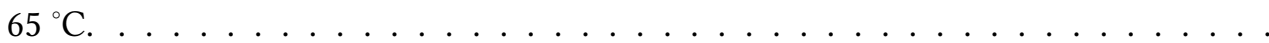

3.17 GIXRD pattern of a $\mathrm{CH}_{3} \mathrm{NH}_{3} \mathrm{PbI}_{3}$ (MAPI) film before and after 1000 cycles of $1 \mathrm{~s}$

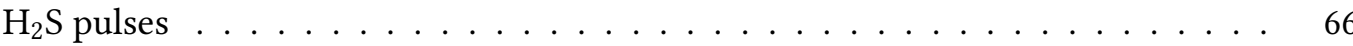

3.18 GIXRD patterns of samples where $\mathrm{PbS}$ was deposited with $\mathbf{0 P b}$ at different temperatures on MAPI films on silicon. . . . . . . . . . . . . . . . 66

3.19 GIXRD of an unprotected MAPI film stored in ambient air. . . . . . . . . . . . . 67

3.20 GIXRD patterns of MAPI films capped with ALD films and stored in ambient air. .

3.21 Oxygen and water transmission rates for BoPET with and without ALD film en-

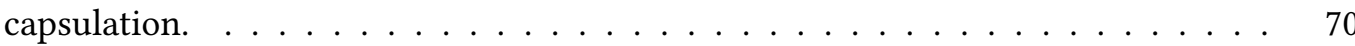

4.1 Known Pb-containing ALD precursors. . . . . . . . . . . . . . . . . 79

4.2 Three-step synthesis of $(\mathbf{4} \mathbf{P b})_{2} \ldots \ldots \ldots \ldots \ldots \ldots \ldots \ldots \ldots$

4.3 Structurally characterized 1,3 -diaza-2-plumba-4-silacyclobutanes. . . . . . . . . 81

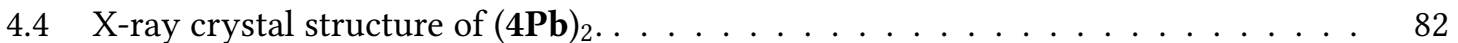

4.5 TGA and vapour pressure of $(\mathbf{4 P b})_{2} \ldots \ldots \ldots \ldots \ldots \ldots \ldots \ldots$

4.6 Atoms-in-molecules (QTAIM) graph and non-covalent interaction (NCI) analysis of $(\mathbf{4 P b})_{2}$ from DFT. . . . . . . . . . . . . . . . . . . 84

$4.7 \quad \mathrm{X}$-ray crystal structure of $\left(\mathbf{4} \mathrm{Pb}_{\mathrm{tPe}}\right)_{2} \ldots \ldots \ldots \ldots \ldots \ldots \ldots \ldots \ldots$

4.8 Calculated relative free energies $\Delta G^{*}$ in $\mathrm{kcal} / \mathrm{mol}$ of dimeric $\left(\mathbf{4} \mathbf{P b} \mathbf{b}_{\mathrm{Et}}\right)_{2}$ and monomeric $4 \mathbf{P b}_{\mathrm{Et}}$ conformers in the gas phase. . . . . . . . . . . . . . . 87

4.9 Proposed adduct formation of $(\mathbf{4 P b})_{2}$ with small readily-available ligands. . . . . . 88

4.10 Atoms-in-molecules (QTAIM) graphs and non-covalent interaction (NCI) analysis of $(\mathbf{4 P b})(\mathbf{L})$ adducts calculated with DFT. . . . . . . . . . . . . . . . 91

4.11 Nitro-Mannich reaction and subsequent aqueous metal reduction of the nitroamine to prepare $\mathrm{H}_{2} \mathrm{NCMe}_{2} \mathrm{CH}_{2} \mathrm{NMe}_{2} \ldots \ldots \ldots \ldots \ldots \ldots$

4.12 Proposed synthesis of $\mathbf{Y}$ and its equilibrium with its $\operatorname{dimer}(\mathbf{Y})_{2} \ldots \ldots \ldots$

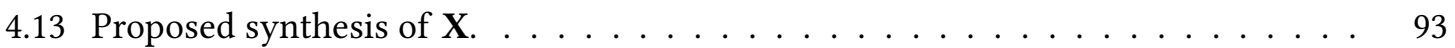

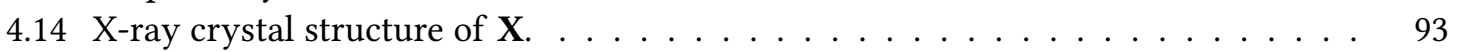

4.15 Atoms-in-molecules (AIM) graphs and non-covalent interaction (NCI) analysis of complex $\mathbf{X}$ and the stable endo conformer of dimeric $(\mathbf{Y})_{2}$ calculated with DFT. . . 95

4.16 Molecular electrostatic potential (MEP) at the van der Waals surface of known $\mathrm{Pb}$ containing ALD precursors and $(\mathbf{4 P b})_{2} \ldots \ldots \ldots \ldots$

4.17 Molecular electrostatic potential (MEP) at the van der Waals surface of the dimer

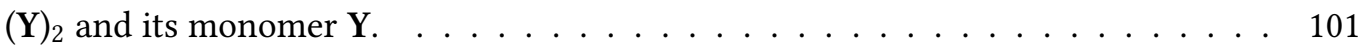

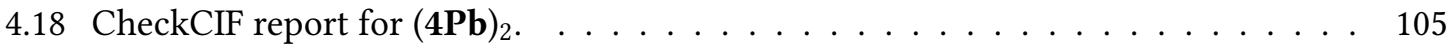

4.19 CheckCIF report for $\left(\mathbf{4} \mathbf{P b}_{\mathrm{tPe}}\right)_{2} \ldots \ldots \ldots \ldots \ldots \ldots \ldots$

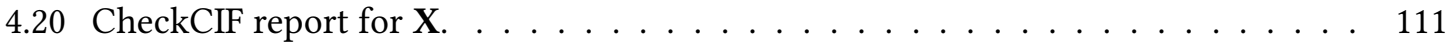

5.1 X-ray crystal structure of 4 Co. . . . . . . . . . . . . . . . . . . 118

5.2 Yang's geometry index $\tau_{4}$ for known tetracoordinate Co(IV) complexes. . . . . . 119

5.3 Experimental and simulated X-band EPR spectra of 4Co. . . . . . . . . . . . . 120

5.4 Orbital diagram of $\mathbf{4 C o}$ in the doublet and quartet ground spin-states. . . . . . . . 122

5.5 Effect on relative energy by sterics in 4 Co analogues. . . . . . . . . . . . . . . 123

5.6 TGA and DSC, vapour pressure, and self-limiting monolayer formation of 4Co. . 125 
6.1 Gas-phase structures of tin(II) bis(trifluoroacetate) (1) and ditin(II) $\mu$-oxy-bis- $\mu$ trifluoroacetate $(2) . \ldots \ldots \ldots$. . . . . . . . . . . . . . . 139

6.2 Gas-phase electron diffraction (GED) radial distribution curves (RDC) for $\mathbf{1}+\mathbf{2}$ (left) and 6 (middle). Quality of least-squares refinement RG/RGmin of GED data of 3 as a function of the mole fraction of $\mathbf{1}$ and $2 \ldots \ldots \ldots$. . . . . . . . . . 141

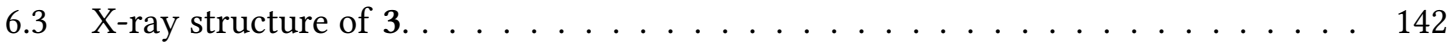

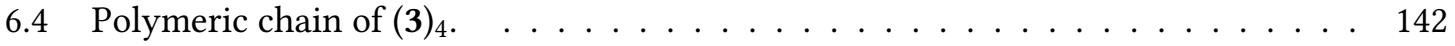

6.5 Crystal packing of a $3 \times 3 \times 3$ supercell of $3 \ldots \ldots \ldots \ldots \ldots \ldots \ldots$

6.6 Crystal packing of a $3 \times 3 \times 3$ supercell of 3 from the top. . . . . . . . . . . 143

6.7 Crystal packing of a $3 \times 3 \times 3$ supercell of 3 from the right. . . . . . . . . . . . . 144

6.8 Extremely distorted pseudosquare pyramidal geometry around each Sn atom in 3. 144

6.9 Asymmetric unit of 3 showing the fate upon evaporation of each Sn atom. . . . . 145

6.10 Frontier orbitals of 1 and 2 calculated by DFT. . . . . . . . . . . . . 146

6.11 Gas-phase structure of $\operatorname{tin}(\mathrm{IV})$ tetrakis(trifluoroacetate) (6) . . . . . . . . . . . 147

6.12 Solid state structure of polymeric 5 . . . . . . . . . . . . . . . . . . 148

6.13 Ramped thermogravimetric analysis (TGA) curves of 3, 7,5 and 8. . . . . . . 149

6.14 Vapour pressure of 3, 7, 2, and $\mathbf{8} \ldots \ldots \ldots \ldots \ldots \ldots$

6.15 Molecular structures $6 \leftarrow\left(\mathrm{OEt}_{2}\right)_{2},\left(1 \leftarrow \mathrm{PMe}_{3}\right)_{6}$, and $4 \ldots \ldots \ldots \ldots \ldots$ 


\section{List of Tables}

3.1 Thermodynamics of possible reactions during ALD of $\mathrm{PbS}$ with $\mathbf{0 P b}$ or $\mathbf{1 P b} . \ldots 52$

4.1 Standard free energies and enthalpies of dimerization and adduct formation $(\mathbf{4 P b})_{2} . \quad 89$

4.2 Mayer bond order (MBO), Wiberg bond index (WBI), and QTAIM topological parameters of $4 \mathbf{P b}$ and its adducts. . . . . . . . . . . . . . . . . . 89

$4.3 \quad$ Vapour pressure and calculated MEP properties of known plumbylenes. . . . . . . 98

4.4 Calculated MEP properties of hypothetical plumbylenes. . . . . . . . . . . . . 100

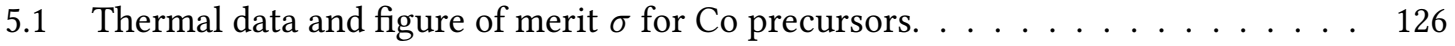




\title{
List of Abbreviations
}

\author{
1GPV First-generation photovoltaic \\ 2GPV Second-generation photovoltaic \\ 3GPV Third-generation photovoltaic \\ AFM Atomic force microscopy \\ AIL Atom interaction lines \\ AIMD Ab initio molecular dynamics \\ ALD Atomic layer deposition \\ AZO Aluminum-doped zinc oxide \\ BOPET Biaxially-oriented poly(ethylene terephthalate) \\ btbas Bis(tert-butylamino)silane \\ CCSD Coupled-cluster with singles and doubles \\ $\operatorname{CCSD}(\mathrm{T}) \quad$ Coupled-cluster with singles, doubles, and perturbative triples \\ CIGS Copper-indium-gallium-selenide \\ COSY Correlation spectroscopy \\ CVD Chemical vapour deposition \\ dad $\quad N, N^{\prime}$-di-tert-butyl-1,4-diazabutadiene \\ DEZ Diethylzinc \\ DFT Density functional theory \\ DKH Douglas-Kroll-Hess \\ DLPNO Domain local-pair natural orbital \\ DOMO Doubly-occupied molecular orbital \\ DSC Differential scanning calorimetry \\ DSSC Dye-sensitized solar cell \\ EA Electron affinity \\ EDS Energy-dispersive X-ray spectroscopy \\ EIMS Electron-impact mass spectrometry \\ FESEM Field-emission scanning electron microscopy \\ FTO Fluorine-doped tin oxide \\ GED Gas-phase electron diffraction \\ GIPF General interactions properties function \\ GIXRD Grazing-incidence X-ray diffraction \\ GPC Growth per cycle \\ HCSC Hot-carrier solar cell \\ HOMO Highest occupied molecular orbital \\ HSQC Heteronuclear single quantum coherence \\ IA Ionization potential \\ iPrNHC 1,3-di-iso-propyl-4,5-dimethylimidazol-2-ylidene
}




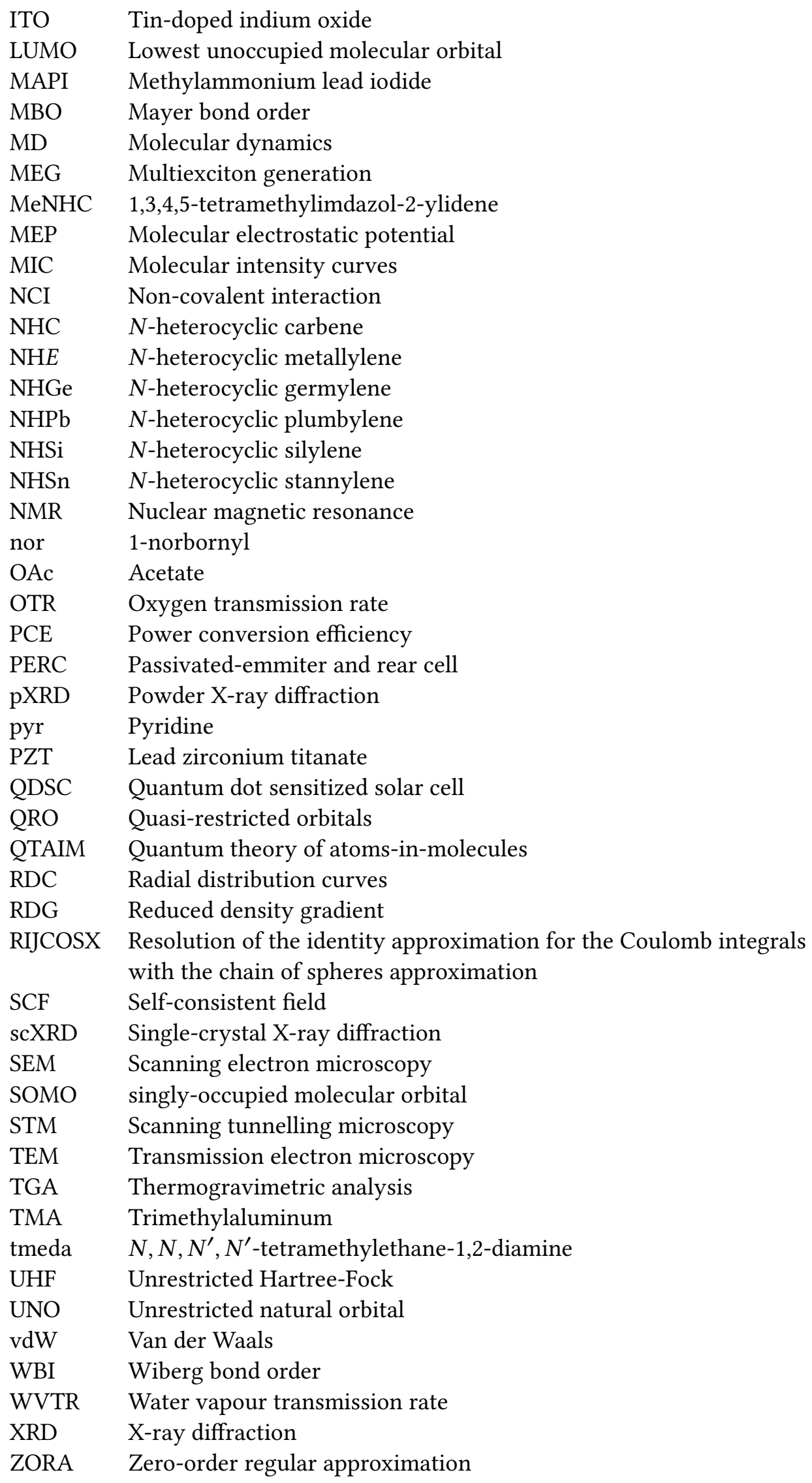


I had this style ever since I was a child

I got this other style I ain't flip in a while, it goes:

Pure scientific intelligence, with one point of relevance

- MF DOOM, Dead Bent, 1971-2020

Although an ordinary man with no skill in poetry, I shall compose some verses, minute as a drop of water, Which will be like a lamp that shines in pitch darkness, And a help to benighted people of ignorance.

- Nāgārjuna, A Drop of Nourishment, ca. 150-250 AD 


\section{Chapter 1}

\section{Introduction}

\subsection{Third-Generation Photovoltaics (3GPVs)}

\subsubsection{Motivation}

Photovoltaics, the conversion of light into electrical potential energy, was first discovered by Becquerel in $1839^{1}$ and first developed into a working technology at Bell Labs in $1954 .^{2}$ This single-crystal silicon $p-n$ junction device represented the archetypical firstgeneration photovoltaic (1GPV) solar cell. ${ }^{3,4}$ Today, commercially-available $1 \mathrm{GPV}$ achieve power conversion efficiencies (PCEs) over $20 \%$, and research devices have reached $26.7 \% .^{5}$ However, these devices are heavy and require a lot of material to produce because silicon is ineffecient at absorbing light and must be thick to achieve good PCE. The packaging required to protect the fragile single-crystals also increases the weight. Second-generation photovoltaics (2GPV) were developed in response by using thin films of materials that absorb light more efficiently like CdTe, ${ }^{6} \mathrm{CuInGaSe}$ (CIGS), ${ }^{7}$ and GaAs. ${ }^{8,9}$ While this strategy reduced weight and raw material cost, the cells were more expensive to manufacture by virtue of their small size and the high-toxicity and scarcity of their constituent elements. This limited the use of 2GPVs to high-performance applications where cost is less of an issue than weight (e.g., aerospace). Third-generation photovoltaics (3GPVs) represent the 
most recent development, and researchers have focused on achieving both low-cost and ultra-high-efficiency. Arguably the first example was the metal-organic dye-sensitized solar cell (DSSC) reported by Grätzel and O'Regan in $1991,{ }^{10}$ and many other material bases and architectures have appeared since then. Some popular approaches are organic photovoltaics (OPVs), ${ }^{11}$ tandem solar cells,${ }^{4}$ and the very recent perovskite solar cells. ${ }^{12}$ Some more exotic approaches have included hot-carrier solar cells (HCSCs), multiexciton generation (MEG), up- and down-conversion, and quantum dot sensitized solar cells (QDSCs). ${ }^{3,4,13-16}$

In an effort to mitigate anthropogenic climate change, cheap solar power is a lowcarbon alternative to fossil fuels and nuclear, and has become the main motivation for the research, development, and commercialization of solar cells from all generations. ${ }^{17,18}$ Its adoption was sluggish due to high cost and low efficiency in the past, and there are other technical hurdles to implementing solar power beyond the cells themselves. Still, the price of solar power has continued to drop while the efficiency rises. The energy demand of industrial society will continue to grow and require cheaper more-efficient solar cells, ${ }^{19}$ which sustains the global interest in new photovoltaic technologies. The need to produce solar cells in large volumes for global power production also means they must be composed of elements that are abundant in the earth's crust, or else they will become prohibitively expensive or unavailable altogether. ${ }^{20,21}$ We were motivated by a perceived lack of atomic layer deposition (ALD) processes to enable the aforementioned emerging $3 \mathrm{GPV}$ technologies.

\subsubsection{Target Materials}

\subsubsection{Transparent-Conducting Materials: F-doped $\mathrm{SnO}_{x}$}

Earth-abundant replacements of the industry standard Sn-doped $\mathrm{In}_{2} \mathrm{O}_{3}$ (ITO) have been subject to intense and prolonged research. Some viable alternatives include conductive 
polymer films (e.g., PEDOT:PSS), encapsulated metallic nanowire films (e.g., Ag), and doped metal oxide films. ${ }^{22}$ While each approach has its advantages, doped metal oxide films are attractive because of the many different formulations that can be deposited by a wide variety of techniques for specific application requirements. The two most promising alternatives are currently Al-doped $\mathrm{ZnO}(\mathrm{AZO})$ and F-doped $\mathrm{SnO}_{x}(\mathrm{FTO}) .^{22,23}$ Thin films of FTO deposited by spray pyrolysis or chemical vapour deposition (CVD) on glass are already ubiquitous commercially-available substrates for photovolatic research. In fact, FTO may even be better for some applications than ITO due to its chemical, thermal, and optoelectronic properties. ${ }^{24}$ However, FTO has yet to replace ITO in advanced applications due to the small number of vapour-phase deposition recipes for it and limited development caused by the hazardous nature of F-doping reagents (e.g., HF). The limited attention given to FTO may also be due to the high-performance and ease-of-deposition of doped $\mathrm{ZnO}$ films, especially by atomic layer deposition (ALD), which is already used in photovoltaics ${ }^{25-27}$ as well as in other optoelectronic applications. ${ }^{28}$ Besides the novelty, we were interested in FTO because, unlike ITO and AZO, it is relatively chemically inert, mechanically robust, and thermally stable, making it compatible with some fabrication processes with which the others are not.

\subsubsection{Light-Absorbing Materials: Pb-based Semiconductors}

$\mathrm{Pb}$-based materials have unique properties that enable exceptionally high-performance photovoltaics. Hybrid organic-inorganic perovskites like methylammonium lead iodide $\left(\mathrm{CH}_{3} \mathrm{NH}_{3} \mathrm{PbI}_{3}, \mathrm{MAPI}\right)$ are notable in particular because of their huge improvement in PCE over a very short period of time (3.8\% to $24.5 \%$ in ca. 10 years). ${ }^{29}$ Unfortunately, their instability under ambient conditions has been a barrier to their industrial application. ${ }^{30,31}$ Two general strategies have emerged to tackle the problem of air- and moisture-sensitivity in perovskites: discovery and design of robust perovskite materials altogether (e.g., $\mathrm{CsPbI}_{3}$ ), and encapsulation. New perovskites, with or without $\mathrm{Pb}$, have gained a lot of atten- 
tion in the literature, but encapsulation of MAPI is especially attractive to industry for practical reasons. All of the progress gained over the last decade can be preserved if the most-studied pervoskite MAPI is used, increasing the likelihood of producing viable commercial devices. Indeed, encapsulated MAPI-containing solar cells are expected to be commercially-available in the near future. ${ }^{30}$ We were interested in perovskites because of the opportunity they presented in photovoltaics and other optoelectronic applications. ${ }^{32-34}$

$\mathrm{PbS}$ and the other chalcogenides are popular and well-studied materials for optoelectronics, but have only seen marginal use in photovoltaic for power generation because of their narrow bandgaps $(\leq 0.4 \mathrm{eV})$ and for historical reasons. ${ }^{36}$ However, they have become hot topics of research because of unusual quantum mechanical properties that make them promising absorbers for 3GPVs. ${ }^{13-16,37,38}$ Quantum confinement, i.e., an increase

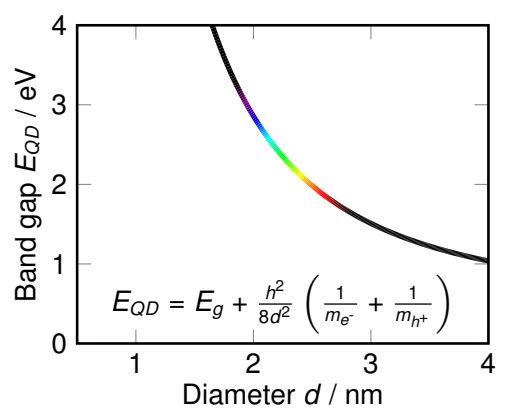

Figure 1.1: Effective mass model of quantum confinement in $\mathrm{PbS}$ nanocrystals. ${ }^{35}$ in the bandgap of a semiconductor when confined in one or more dimensions below a certain size, is particularly effective with $\mathrm{PbS}$ because its large exciton Bohr radius $(23 \mathrm{~nm})$ and small bulk bandgap $(0.43 \mathrm{eV})$ allows tuning the absorption energy from the ultraviolet to the infrared. Other benefits of quantum confinement include, for example, slowed carrier cooling and recombination, which may become critical to ultra-high-efficiency 3GPVs in the future. ${ }^{39}$ Multiexciton generation (MEG), i.e., where one high-energy photon can be converted to two or more excited electron-hole pairs by the process of Auger excitation, is especially efficient in the $\mathrm{Pb}$ chalcogenides, and raises the possibility of capturing the large portion of energy lost to heat in most solar cell designs. ${ }^{15,16}$ Besides its unusual properties, $\mathrm{PbS}$ also has attractive conventional properties for photovoltaics including chemical inertness, thermal stability, and rare $p$-type conductivity. ${ }^{36}$ We were interested in ALD of $\mathrm{PbS}$ to fine-tune its bandgap by subnanometer 
thickness control over its size and morphology, and in its functional application alongside MAPI.

\subsection{Atomic Layer Deposition (ALD)}

\subsubsection{Mechanism}

Atomic layer deposition (ALD) is a stepwise variation of the continuous chemical vapour deposition (CVD) method where layer-by-layer growth is controlled entirely by the surface chemistry between two or more co-reactants. ${ }^{40-42}$ The critical step in ALD is the formation of a selflimiting monolayer of chemisorbed precursor molecules on the surface which do not undergo further reaction with additional vapour-phase precursors. Excess unreacted precursor molecules are then removed by pumping and purging with inert gas, leaving behind the monolayer which can then be reacted with one or more

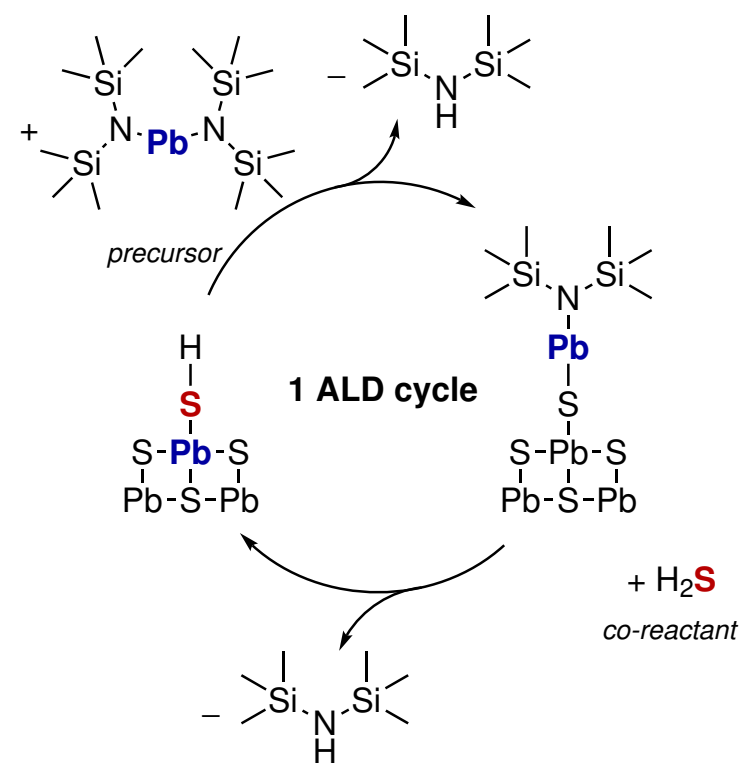

Figure 1.2: Diagram of a single atomic layer deposition (ALD) cycle. First, the surface reacts with the precursor $\mathrm{Pb}\left[\mathrm{N}\left(\mathrm{SiMe}_{3}\right)_{2}\right]_{2}(\mathbf{O P b})$ to form the self-limiting monolayer $\|-\mathrm{S}-\mathrm{Pb}-\mathrm{N}\left(\mathrm{SiMe}_{3}\right)_{2}$ and eliminate the free amine $\mathrm{HN}\left(\mathrm{SiMe}_{3}\right)_{2}$. Then, the co-reactant $\mathrm{H}_{2} \mathrm{~S}$ reacts with the self-limiting monolayer to form the desired material $\mathrm{PbS}$, and the original surface is regenerated.

vapour-phase co-reactant(s). These steps

constitute sub-cycles of one complete ALD cycle. This self-limiting behaviour is also referred to in the art as "saturation," and each precursor or co-reactant must display saturation with respect to its exposure to the surface for a process to truly be ALD. The thickness and quality of the film deposited per ALD cycle is determined entirely by the specific process surface chemistry, making ALD particularly sensitive to the nature of the 
precursor molecules and their reactivity with co-reactants.

The sequential reaction of precursor and co-reactant is achieved by separation in time between purges with an inert gas, as is done in conventional ALD; or in space, by moving the substrate or reactor between flowing streams of precursor, inert gas, and co-reactants to sequentially expose the surface to each, as is done in spatial ALD. ${ }^{43,44}$ Conventional ALD is amenable to batch processing and high-surface area powders; while spatial ALD is amenable to continuous or large-area deposition. Recently, ALD-like processes have also been demonstrated in the solution-phase using a microfluidic device, ${ }^{45,46}$ by sequential injection of precursors to a suspension of particles, ${ }^{47}$ phase-transfer between polar and non-polar media, ${ }^{48}$ and by reversal of the mobile and stationary phases during deposition by using ion-exchange resins for the precursors and flowing colloidal nanoparticle solutions for the substrates. ${ }^{49}$ In this work, we have limited ourselves to conventional ALD (herein simply "ALD") because of its long history and intimate connection to industry, ${ }^{50-53}$ and its demonstrated ability to coat high-aspect ratio or tortuous nanostructures. ${ }^{54-56} \mathrm{Ad}-$ ditional practical reasons are the availability, low-cost, simple operation, and popularity of conventional ALD reactors, ${ }^{57}$ which means they are more likely to be ready-to-hand for researchers in academia and industry, thus increasing the impact of this work.

\subsubsection{Growth Behaviour \& Thin Film Quality}

While the details of ALD growth behaviour and thin film quality are specific to each process chemistry and conditions, there are a number of features that ALD has become well-known for.

\subsubsection{Subnanometer Thickness Control}

Thickness of films deposited by ALD at a given temperature are linearly dependent on the number of cycles performed and independent of total deposition time, thus there is no proper rate of deposition but a "growth per cycle" (GPC). Deviation from this linear 
growth mode is common at few cycles, manifesting as a nucleation delay when growth is retarded and nucleation enhancement when growth is accelerated. Nucleation delay or enhancement can be caused by differences in reactivity of the precursor with the substrate surface, surface roughness, packing efficiency of the self-limiting monolayer, crystallinity of the nucleating and growing films, or even by deliberate pre-treatment of the surface. ${ }^{58,59}$ The practical upshot of the linear dependence of thickness on cycle number is that the film thickness can be chosen by the user simply by applying the correct number of ALD cycles.

\subsubsection{Uniformity \& Conformality}

ALD films are very uniform across large substrate areas because of its self-limiting mechanism. More importantly, they conform to the nano-, micro- and macroscopic shape of the substrate. ${ }^{54-56}$ Not only does this mean that ALD does not require line-of-sight and can coat virtually any shape or size, its conformality can approach $100 \%$ and allow uniform deposition down deep vias ${ }^{55}$ and on tortuous structures like photonic crystals. ${ }^{56}$ This feature in particular made ALD an indispensable method for the continued miniaturization of feature sizes in memory and logic devices. ${ }^{60-62}$

\subsubsection{Interface \& Composition Control}

The sequential, self-limiting nature of ALD lends itself to the deposition of films with tunable interfaces and compositions. ${ }^{63}$ This includes routine synthesis of doped (e.g., Aldoped $\mathrm{ZnO}, \mathrm{AZO}$ ) and ternary materials (e.g., $\mathrm{PbTi}_{x} \mathrm{O}_{y}$ ), and less often quaternary and higher materials (e.g., $\operatorname{LiLa}_{w} \mathrm{Zr}_{x} \mathrm{Al}_{y} \mathrm{O}_{z}$ ). Perfect epitaxy can be achieved by ALD with the right process and substrate, ${ }^{64-66}$ but near-perfect heterojunction interfaces can also be

achieved with non-epitaxial deposition. ${ }^{67}$ The vast catalogue of existing ALD recipes ${ }^{68-70}$ and their mixtures presents the opportunity for every functional layer of a photovoltaic device to be fabricated entirely from the bottom-up by ALD. 


\subsubsection{ALD \& 3GPVs}

One of the most substantial performance improvements in 1GPVs was recently enabled by $\mathrm{ALD} \mathrm{Al}_{2} \mathrm{O}_{3}$ thin films that provided excellent passivation of the $p$-type emitter of passivated-emitter and rear cell (PERC) Si solar cells, ${ }^{71}$ which quickly became a common passivation method for commercial 1GPVs. ${ }^{72}$ For 2G- and 3GPVs, the future of ALD in photovoltaics is very promising and has been reviewed extensively in the literature. ${ }^{73-80}$ In this work, we were especially motivated by future devices with ultrathin absorbers. Ultrathin films on realistic high-aspect ratio nanowire arrays can have the same volume (thus the same absorption cross-section) as much thicker monolithic slabs typical
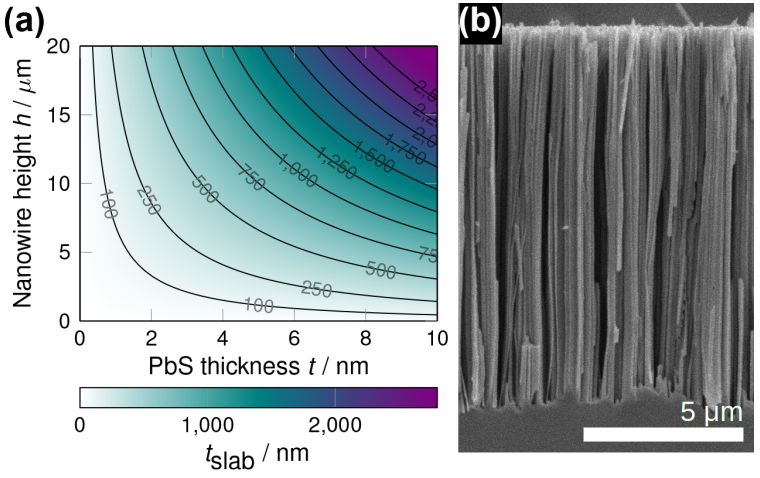

(c)

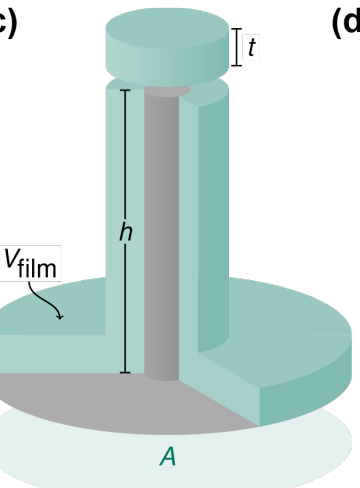

(d) $v_{\text {slab }}$

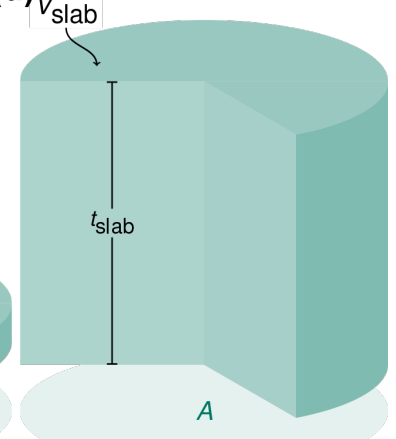

Figure 1.3: (a) Equivalent slab volume of ultrathin absorbers of thickness $d$ on nanowire arrays of height $h$. (b) Silicon nanowire array. (c) Diagram of conformally coated nanowire and its volume $V_{\text {film }}$ and (d) of a monolithic slab and its volume $V_{\text {slab }}$ (not to scale). in thin film 2GPVs (Figure 1.3). However, suitable precursors for FTO and $\mathrm{Pb}$-containing materials did not exist before this work.

\subsection{Precursor Design}

Atomic layer deposition (ALD) would not be possible without suitable precursors, but there is no systematic methodology called precursor design. Instead, a variety of design strategies have emerged: from synthetic exploration, ${ }^{81}$ to literature review, ${ }^{82,83} \mathrm{di}$ rected evolution, ${ }^{84,85}$ and computational screening protocols. ${ }^{86-91}$ Historical perspectives 
on these design strategies were collected in a dedicated issue of Coordination Chemistry Reviews, ${ }^{92}$ and the state-of-the-art was recently reviewed by Johnson and Parish. ${ }^{93}$ All ALD precursors share the same performance criteria and the three most important are: volatility, thermal stability, and chemical reactivity. Here, we will introduce specific reasons and requirements for each.

\subsubsection{Performance Criteria}

\subsubsection{Volatility}

Volatility is required to evaporate the precursor into the vapour-phase, transfer it to the deposition chamber to react with the substrate, remove unreacted excess precursor and by-products, and finally leave behind a self-limiting monolayer for the next subcycle. There is no upper limit on volatility from a practical standpoint, and some metalcontaining precursor molecules are even gaseous under standard conditions (e.g., $\mathrm{WF}_{6}$ ). The lower limit on volatility is determined by the deposition conditions (e.g., temperature and pressure) and thermal budget of the specific ALD reactor and application. Under usual circumstances, the precursor must achieve a 1 Torr vapour pressure below $150{ }^{\circ} \mathrm{C}$ because ALD reactors typically operate at 1 Torr pressure under flow and precursor-delivery systems (e.g., bubblers, ampules) usually cannot be heated above $150{ }^{\circ} \mathrm{C}$. More generally, the precursor should achieve a vapour pressure of about the base pressure of the ALD reactor at a temperature below the desired deposition temperature so that it does not condense onto colder areas of the delivery line, reactor, or exhaust. If this were to occur, the excess unreacted precursor could not be effectively removed from the deposition chamber and continuous surface- and gas-phase reactions (CVD) would occur. In general, the more volatile a precursor is, the better.

The physical requirements of volatility for CVD and ALD precursors are straightforward, but the physical mechanism of vaporization itself is not. There is no systematic 
methodology to design volatile molecules and the chemical features that determine the volatility of even simple molecules are not well understood. Evaporation and condensation have been the subject of intense research for over 130 years due to their importance in a great number of physical sciences, but theoretical and experimental difficulties persist. ${ }^{94}$ Even the measured evaporation and condensation coefficients of water in the literature spanned three orders of magnitude until recently. While the precise details of the problem are outside the scope of this work, a few key points warrant introduction to give perspective on its relevance to precursor design. First, it should be stressed that evaporation involving mass transfer, such as precursor delivery for ALD, is a non-equilibrium process, ${ }^{95}$ so the familiar textbook thermodynamics of phase changes (i.e., Clausius-Clapeyron $p-\mathrm{T}$ relation) are at best an approximation. Consequently, equilibrium vapour pressure data such as normal boiling points are not truly applicable, despite the art's frequent reliance on and reference to such data. The microscopic mechanisms of evaporation phenomena are not clear, so molecular dynamics (MD) simulations are key to probing their nature atomistically, and they have highlighted the complexity of the process. ${ }^{96-101}$ For example, one study of water revealed most molecules evaporated by a surprising mechanism involving three molecules cooperating at once. ${ }^{98}$ By extension, it is unclear which mechanisms may describe metal-containing precursor evaporation. It is becoming feasible to simulate evaporation of technologically relevant organic molecules directly with MD with better access to high-performance computing facilities, ${ }^{101}$ but these methods rely on force-fields that are unavailable or inaccurate for metal-containing species like ALD precursors. Ab initio molecular dynamics (AIMD) simulations using quantum chemical calculations at each step can circumvent this problem, but they have currently only been applied to the evaporation of simple metal-containing molecules (e.g., $\mathrm{MgSiO}_{3}$ ). ${ }^{100}$ To summarize, there is a substantial amount of physics required before volatility can be rationally designed into precursors. 


\subsubsection{Thermal Stability}

Precursor molecules must be thermally stable for technical and practical reasons. Technically, they must survive heating so that they may be evaporated and used for ALD, and their self-limiting monolayers must also be thermally stable so that continuous CVD does not occur. Practically, they must have sufficient thermal stability so that they can survive prolonged heating without decomposition. Even if a precursor decomposes slowly at temperatures required for evaporation, the change in composition and vapour pressure over time will cause unacceptable variability during deposition and between batches. While this is not an issue in academic settings where the precursor may be refreshed between depositions, ALD processes are often developed with a technological application in mind, so industrial requirements cannot be entirely ignored.

\subsubsection{Chemical Reactivity}

Chemical reactivity is primarily required so that the precursor reacts with the surface to form a self-limiting monolayer which then reacts with the co-reactants to deposit a thin film of the desired material. Thus the chemical reactivity of the precursor is coupled to the co-reactants, the deposition conditions, the substrate, and the material that is to be deposited. For example, even if a precursor is volatile and stable during evaporation, it may still undergo CVD on a surface, be inert toward available co-reactants, introduce impurities into the growing film, or deposit the wrong material entirely. Thus the expected products of the ALD half-reactions are as much a part of the precursor as the molecule itself, and they must be considered together to effectively design a precursor. The highreactivity desired in ALD precursors so that they undergo fast, complete reactions at low temperatures means the precursors often pose a serious health and safety risk. For example, two of the most popular ALD precursors trimethylaluminum (TMA) and diethylzinc (DEZ) are pyrophoric and react violently with moisture in ambient conditions to produce heat and methane or ethane gas, which can cause a fire or even an explosion. Both 
must be handled and stored under air-free conditions at all times to prevent accidents. Less reactive non-pyrophoric ALD precursors are still often air- and moisture-sensitive, so they too must be handled under air-free conditions to prevent decomposition. There are also secondary requirements for reactivity that are more integrated with the rest of the ALD process than volatility; these include handling, storage, cost, environmental impact of synthesis and disposal, compatibility with the reactor and its components (e.g., stainless steel, O-rings), and compatibility with the substrate (i.e., to avoid etching). The finer points of the entire precursor design process from conceptualization to industrialization lie outside the scope of this work, but have been discussed in the literature.

\subsubsection{Thermogravimetric Analysis (TGA)}

Thermogravimetric analysis (TGA) is a precise, accurate, and cost-effective method to evaluate the thermal stability and volatility of ALD precursors. The technique involves measuring the mass of a sample over time while heating, either in an isothermal or non-isothermal mode, under a flow of gas, and the apparatus is made up of a sensitive electronic balance and thermocouple housed in a computer-controlled high-temperature furnace. It can be used to quantitatively investigate complex kinetics of solid-state reactions, but we are particularly interested in quantifying the vapour pressure and decomposition temperatures of precursors. There is a well-known and theoretically justified method to estimate the vapour pressure from the rate of mass loss according to the Langmuir

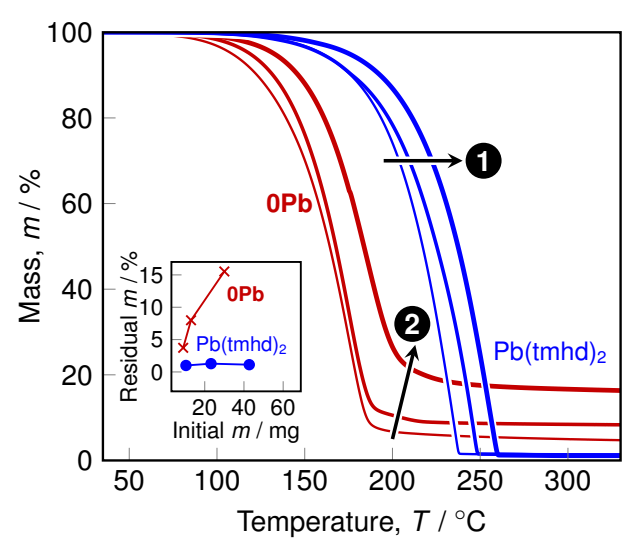

Figure 1.4: Thermogravimetric analysis (TGA) of two $\mathrm{Pb}$-containing precursors with increasing initial mass (ca. 10, 20 , and $40 \mathrm{mg}$ ) constituting a stress-test. The precursor $\mathrm{Pb}(\mathrm{tmhd})_{2}$ takes longer to evaporate (1) but its residual mass does not increase (inset), therefore it is stable. In contrast, $\mathbf{0 P b}$ decomposes as the initial mass is increased because it takes longer to evaporate and is heated to higher temperatures where it leaves behind non-volatile residue (2), therefore it is unstable. 
equation for evaporation in vacuum (Equation 1.1). ${ }^{102}$

$$
-\frac{\mathrm{d} m}{\mathrm{~d} t}=p \alpha_{\mathrm{vap}} \sqrt{\frac{M}{2 \pi R T}}
$$

where $-\mathrm{d} m / \mathrm{d} t$ is the rate of mass loss per unit area, $p$ the vapour pressure, $M$ the molecular weight of the vapour, $R$ the gas constant, $T$ the temperature, and $\alpha_{\text {vap }}$ is the vaporization coefficient. Under vacuum, $\alpha$ is typically taken to be unity, but this cannot be assumed under flowing gas at ambient pressures. We can rearrange Equation 1.1 in terms of the pressure $p$ as follows

$$
p=-\left(\frac{\sqrt{2 \pi R}}{\alpha_{\text {vap }}}\right)\left(\frac{\mathrm{d} m}{\mathrm{~d} t} \sqrt{\frac{T}{M}}\right)=-k v
$$

where $k=\sqrt{2 \pi R} / \alpha_{\text {vap }}$ becomes a constant for a particular TGA instrument that can be calibrated by measuring the weighted rate of mass loss $v=\mathrm{d} m / \mathrm{d} t \sqrt{T / M}$ for compounds of known vapour pressure. ${ }^{102}$ This technique was first used by Kunte et al. to characterize the vapour pressures of MOCVD precursors in $2008^{103}$ and has become indispensable in our group for the characterization of novel potential ALD precursors. Indeed, the accuracy of the vapour pressure measurements for precursors is demonstrated by the fact that the estimated 1 Torr temperature often is a suitable delivery temperature for precursors in different reactors.

Besides estimating the vapour pressure, there is another simple TGA technique, developed in our group, that allows for the qualitative evaluation of the thermal stability of precursor molecules. In the so-called "thermal-stress test" the initial mass loading is incrementally increased so that it takes longer for the sample to evaporate. Thus, for higher mass loadings, more sample will be heated to higher temperatures where decomposition events might occur. Since precursors usually decompose to non-volatile materials, the residual mass after the experiment will correlate with the initial mass loading, indicating that decomposition has occurred. The onset of these decomposition events can be 
estimated by analyzing the derivative of the TGA curve because evaporation is a zerothorder kinetic process (i.e., it does not depend on the "concentration" of the sample). Since concentrations make no sense for neat solids and liquids, the so-called extent of reaction $\alpha_{\text {ext }}(t)$ is used instead (Equation 1.3).

$$
\alpha_{\mathrm{ext}}(t)=\frac{m_{i}-m(t)}{m_{i}-m_{f}}
$$

where $m_{i}$ is the initial mass, $m_{f}$ is the final mass after the TGA experiment, and $m(t)$ is the mass at time $t$ during the experiment. With careful experimentation and analysis, the kinetics of a reaction can be determined by TGA. ${ }^{104}$ Since evaporation is a zerothorder kinetic process, the derivative of extent of reaction with respect to time under nonisothermal conditions will be a negative exponential if the sample is undergoing evaporation exclusively. ${ }^{105-107}$ Thus reactions such as melting or decomposition that overlap with evaporation will cause deviation from this ideal shape beginning at their onset. ${ }^{108}$ Overall, the shape of the derivative curve from TGA experiments is helpful for qualifying the thermal stability of precursor molecules. 


\section{Chapter 2}

\section{Designing Stability Into Thermally Reactive Plumbylenes}

Reprinted and adapted from the original article published as:

Goran Bačić, ${ }^{c}$ David Zanders, ${ }^{c, b}$ Bert Mallick, ${ }^{b}$ Anjana Devi, ${ }^{b}$ and Seán T. Barry. ${ }^{c}$ Designing Stability Into Thermally Reactive Plumbylenes, Inorg. Chem, 2018, 57, 14, 8218-8226. Copyright 2018 American Chemical Society.

c Department of Chemistry, Carleton University, 1125 Colonel By Drive, Ottawa, Ontario, K1S 5B6, Canada

$b$ Inorganic Materials Chemistry, Faculty of Chemistry and Biochemistry, Ruhr University Bochum, Universitätsstraße 150, 44801, Bochum, Germany 


\section{Abstract}

Lead analogues of $\mathrm{N}$-heterocyclic carbenes (NHPbs) are the least understood members of this increasingly important class of compounds. Here we report the design, preparation, isolation, structure, volatility and decomposition pathways of a novel aliphatic $\mathrm{NHPb}$ : rac- $N,{ }^{2} N^{3}$-di-tert-butylbutane-2,3-diamido lead(II) (1Pb). The large steric bulk of the tertbutylamido moeities and rac-butane backbone successfully hinder redox decomposition pathways observed for diamidoethylene and -ethane backbone analogues, pushing the onset of thermal decomposition from below $0{ }^{\circ} \mathrm{C}$ to above $150{ }^{\circ} \mathrm{C}$. With an exceptionally high vapour pressure of 1 Torr at $94 \pm 2{ }^{\circ} \mathrm{C}$ and excellent thermal stability among $\mathrm{Pb}$ (II) complexes, $\mathbf{1 P b}$ is a promising precursor for the chemical vapour deposition (CVD) and atomic layer deposition (ALD) of functional lead-containing materials. 


\subsection{Introduction}

Lead-containing materials like ferroelectric $\mathrm{Pb}(\mathrm{Zr}, \mathrm{Ti}) \mathrm{O}_{3}(\mathrm{PZT})$, organolead halide perovskites (e.g. $\mathrm{CH}_{3} \mathrm{NH}_{3} \mathrm{PbI}_{3}$ ), and lead (II) chalcogenides (e.g. PbS) are high-performance functional materials for next-generation memory ${ }^{109}$ and optoelectronic devices. ${ }^{15,16,35,110-112}$ Future device architectures will require subnanometer thickness control of functional layers, emphasizing the need for accessible lead-containing precursors for fabrication techniques like chemical vapour deposition (CVD) and atomic layer deposition (ALD). However, known precursors are scarce, and available chemical precursors are either too hazardous (e.g. $\mathrm{Et}_{4} \mathrm{~Pb}$ ) or non-volatile (e.g $\mathrm{Pb}(\mathrm{II}) \beta$-diketonates) ${ }^{113}$ to be practical in either an industry or research setting. The most common lead-containing vapour phase precursor, lead (II) bis(2,2,6,6tetramethyl-3,5-heptanedionate) $\left[\mathrm{Pb}(\mathrm{tmhd})_{2}\right]$, only achieves a vapour pressure of 1 Torr at a relatively high temperature of $166^{\circ} \mathrm{C},{ }^{114}$ making it difficult to use in commercial reactors that operate at approximately 1 Torr. This problem is exacerbated by the small available library of volatile $\mathrm{Pb}$ complexes to choose as potential chemical precursors, and manifests in relatively infrequent reports of ALD of lead-containing materials. ${ }^{70}$

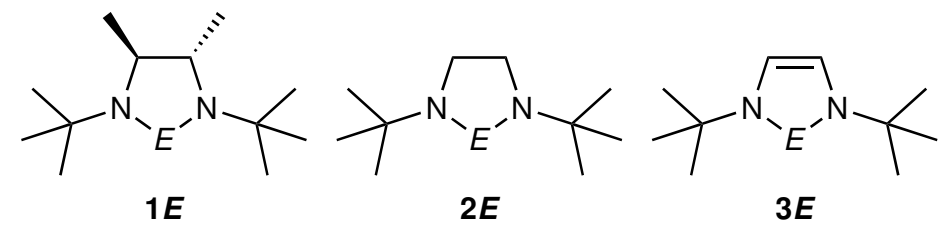

Figure 2.1: Notation for small divalent $N$-heterocyclic metallylenes (NHEs) investigated in this work. Backbone functionalization is denoted as follows: dimethylated $(\mathbf{1 E})$, hydrogenated $(\mathbf{2 E})$, and unsaturated $(3 E)$ where $E=\mathrm{Si}-\mathrm{Pb}$.

$N$-heterocyclic metallylenes (NHEs, where $E=\mathrm{Si}-\mathrm{Pb}$, Figure 2.1) are an emerging class of compounds that are competent and versatile ligands for homogeneous catalysis, ${ }^{115-117}$ as well as volatile, thermally stable and chemically reactive precursors for CVD ${ }^{118}$ and ALD. ${ }^{119}$ Although 2Ge, ${ }^{118} \mathbf{1 G e}$ and $\mathbf{1 S n}{ }^{119}$ have seen significant employment as vapourphase precursor compounds, the corresponding lead analogues (NHPbs) have not received 
the same attention. The number of examples of these compounds decreases when descending the group: $\mathbf{1 P b}$ is claimed in a patent, ${ }^{120}$ but no physical, structural or spectroscopic details have been reported; analogues of $\mathbf{2} \mathbf{P b}$ with aryl or $\mathrm{SiMe}_{3}$ amido functionalization have been reported and characterized, but no thermal data have been presented; and to date there is only one other (failed) attempt to prepare $\mathbf{3 P b} .^{121}$

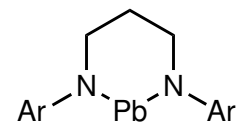

A

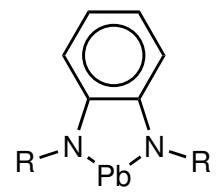

B

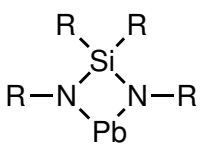

C

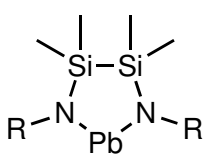

D

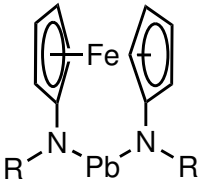

E

Figure 2.2: $\beta$ - (A), benzannulated (B), monosilyl- (C), disilyl- (D), and ferrocenyl (E) diamido plumbylenes.

Other $\alpha$ - and $\beta$-diamido $\mathrm{Pb}(\mathrm{II})$ complexes have been reported in the literature (Figure 2.2). Monomeric aryl- $\alpha$ - and $\beta$-amido (2Pb and $\mathbf{A})$ and benzannulated (B) NHPbs display significant intermolecular interactions via $\pi$ - $\pi$ stacking. ${ }^{122,123}$ While other derivatives of $\mathbf{2} \mathbf{P b}$ are often dimeric with bulky, electron-rich aryl ${ }^{123}$ or $\mathrm{SiMe}_{3}{ }^{124}$ groups. Dimerization can impart significant disadvantages on the volatility of discrete molecular compounds due to the relationship between vapour pressure and molecular weight, and is best avoided in the design of precursors for heavy metals like $\mathrm{Pb} .{ }^{125}$ No thermal data were reported for compounds of type A, B, or $\mathbf{E}$ and none were purified by sublimation or distillation. Silyl-backbone (C, D) plumbylenes have been reported to decompose considerably when distillation or sublimation was attempted. ${ }^{126-128}$

We considered previously reported NHPb species unsuitable as potential precursors, so we sought to prepare $\mathbf{1 P b}, \mathbf{2} \mathbf{P b}$ and $\mathbf{3 P b}$ analogues as potentially monomeric, volatile, and thermally robust precursors for ALD. We were motivated to explore the thermal chemistry of a relatively unknown family of aliphatic $\mathrm{NHPb}$ analogues due to their low molecular weight, ease of synthesis, resistance to dimerization, ${ }^{129}$ as well as the overall lack of practical precursors for ALD of lead-containing materials. Here, we report the at- 
tempted syntheses and decomposition pathways of two $\mathrm{NHPb}$ derivatives $2 \mathbf{P b}$ and $\mathbf{3 P b}$, and the successful preparation, structural analysis, and thermal characterization of $r a c-N^{2}$ , $N^{3}$-di-tert-butylbutane-2,3-diamido lead(II) (1Pb).

\subsection{Results and Discussion}

\subsubsection{N, $N^{3}$-di-tert-butylethylene-2,3-diamido lead(II) (3Pb)}

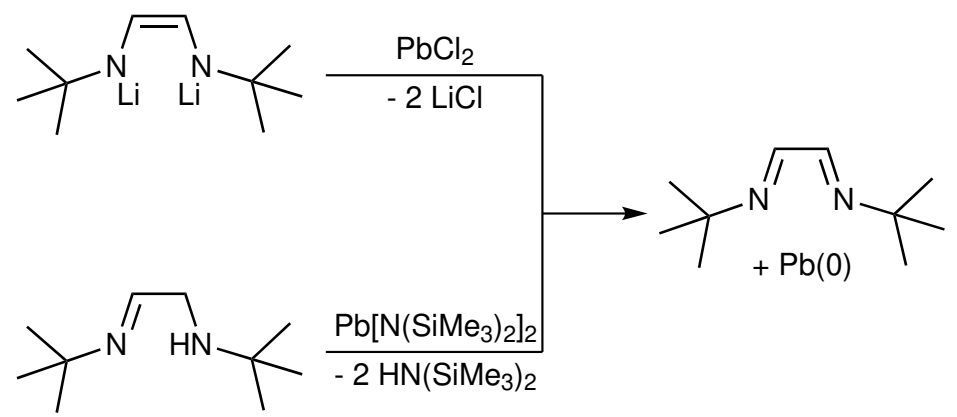

Figure 2.3: $[4+1]$ cycloreversion upon attempted syntheses of $\mathbf{3 P b}$ by salt metathesis with dilithiated diazadienide or transamination with $N, N^{\prime}$-di-tert-butyl-1-aldimino-2-amine. The resulting diimine can be recovered in high yield by sublimation directly from the residue.

First, we reproduced literature reports of decomposition preventing the synthesis of 3Pb by salt-metathesis with $N, N^{\prime}$-di-tert-butyl- $N, N^{\prime}$-dilithiodiazadienide, or by transamination with $N, N^{\prime}$-di-tert-butyl-1-aldimino-2-amine. ${ }^{121}$ Both pathways resulted in the precipitation of black particles (inferred to be $\mathrm{Pb}(0)$ ) at $-78{ }^{\circ} \mathrm{C}$ (Figure 2.3), the parent $N, N^{\prime}$-di-tert-butylethylene-1,2-diimine could be recovered in high yield (>90\%) by sublimation directly from the residue, or after filtration, and removal of solvent. Low-temperature isolation of the target $\mathbf{3 P b}$ was not attempted as decomposition occured far below room temperature. The intermediate (IIPb, below) was reported to decompose before forming the heterocycle. ${ }^{121}$

The instability of $\mathbf{3 P b}$ is in stark contrast to the exceptionally robust $\mathbf{3 S i}$ and $\mathbf{3 G e}$, which thermolyze at 220 and $626{ }^{\circ} \mathrm{C}$, respectively. ${ }^{118,130}$ However, 3Sn is reported to decompose at a milder $60{ }^{\circ} \mathrm{C},{ }^{121}$ supporting an increasingly non-innocent character of the di- 


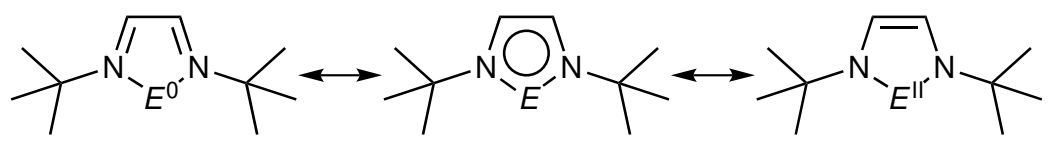

Figure 2.4: Unsaturated $N H E s \quad 3 E$ are $6 \pi$-aromatic rings. Historically, and for the lighter congeners, the ylene character (right) is emphasized over the ylone (left). The zero-valent ylone description becomes increasingly important when descending the group.

imine ligand when descending the group. Historically, the formally zero-valent (i.e. ylone, chelated atom) descriptions of $\mathbf{3} \boldsymbol{E}$ derivatives were considered exaggerations of aromatic resonance, ${ }^{131}$ and the compounds are typically depicted as divalent ylenes. However, recent attempts to form $3 E \rightarrow \mathrm{AeCp}_{2}^{*}$ coordination complexes of the alkaline earth metals $(\mathrm{Ae}=\mathrm{Ca}-\mathrm{Ba})$ with $3 \mathrm{Si}$ and $3 \mathrm{Ge}$ produced the $N, N^{\prime}$-di-tert-butylethylene-1,2-diimine adducts $\mathrm{AeCp}_{2}^{*}(\mathrm{DAD})$ and crystalline $\mathrm{Si}(0)$ and $\mathrm{Ge}(0)$, respectively, even at $-40{ }^{\circ} \mathrm{C} .{ }^{132}$ The unusual formal reduction to elemental $\mathrm{Si}$ and Ge was rationalized as a consequence of a significant zero-valent character of $\mathbf{3 S i}$ and $\mathbf{3 G e}$, which permitted the transfer of the diimine ligand to $\mathrm{AeCp}_{2}^{*}$ from a naked $\mathrm{Si}$ or Ge atom. Spontaneous naked atom transfers in solution were observed by NMR spectroscopy for 3Ge, 3Sn, and derivatives with other diimines. ${ }^{121,133}$ Unlike its lighter congeners, the solely ylone character of $3 \mathbf{P b}$ prevents its isolation.

\subsubsection{N, $N^{3}$-di-tert-butylethane-2,3-diamido lead(II) (2Pb)}

We then attempted to prepare the saturated $\mathbf{2} \mathbf{P b}$ by transamination of lead(II) bis[bis(trimethylsilyl)amide] $(\mathbf{O P b})$ with free $N, N^{\prime}$-di-tert-butylethylene-1,2-diamine in toluene. Turbidity developed immediately upon addition of diamine and within minutes a dark precipitate had formed. After the mixture was stirred for two days in the dark, ${ }^{1} \mathrm{H}$ and ${ }^{13} \mathrm{C}$ NMR spectroscopy revealed no starting $\mathbf{0 P b}$ remained and signals for $N, N^{\prime}$-di-tertbutylethylene-1,2-diimine were observed. $2 \mathrm{Ge}$ was reported to decompose by bimolecular formal dehydrogenation at elevated temperatures, ${ }^{118,134}$ which we suspected occured readily at room temperature with $\mathrm{Pb}$. To investigate further, we performed in-situ ${ }^{1} \mathrm{H}$ NMR 
spectroscopy during the reaction to try and elucidate the decomposition pathway. We propose that the formation of $\mathbf{2} \mathbf{P b}$ is prevented by a stepwise bimolecular dehydrogenation mechanism through a monosubstituted $\mathbf{I P b}$ and the previously observed IIPb, ${ }^{121,135}$ shown in Figures 2.5 and 2.6. Another possible unimolecular pathway for the formation of IISn and IIPb was described previously. ${ }^{121}$

Starting reactants were consumed rapidly and were undetectable the next day; while signals very similar to the tert-butyl groups $[\delta=1.35 \mathrm{ppm}(\mathrm{br}, 9 \mathrm{H})$ and $\delta=1.03 \mathrm{ppm}$ (br, 9H)] of the 3-coordinate intermediate $\mathbf{I I P b} \mathbf{P}^{121}$ appeared within a few minutes and increased over the duration of the experiment. It is likely the chemical shifts of the analogous groups on $\mathbf{I P b}$ are indistinguishable from the oxidized IIPb considering the peakbroadening from poor shimming due to precipitation of $\mathrm{Pb}(0)$ as the reaction proceeded, and the integrated area of signals in the region expected for $-\mathrm{N}\left(\mathrm{SiMe}_{3}\right)_{2}$ on $\mathbf{I P b}$ and $\mathbf{I I P b}$ remained constant during the experiment. We suspect the diamine released by decomposition of $\mathbf{I P b}$ reacts with further $\mathbf{0 P b}$ to replenish its concentration, and the complete consumption of starting diamine during the reaction before decomposition of the intermediates suggests the formation of $\mathbf{I P b}$ and $\mathbf{I I P b}$ is favoured over the formation of $\mathbf{2} \mathbf{P b}$ and $\mathbf{3 P b}$, respectively, analogous to the preparation of IISn and 3Sn. ${ }^{121,135}$ The signals corresponding to $\mathrm{N}=\mathrm{CH}(\delta=8.08 \mathrm{ppm})$ imino and tert-butyl protons $(\delta=1.17 \mathrm{ppm})$ on $N, N^{\prime}$-di-tert-butylethylene-1,2-diimine increased monotonically, and after 5 days free $\mathrm{HN}\left(\mathrm{SiMe}_{3}\right)_{2}$, diamine and diimine were the only detectable species by ${ }^{1} \mathrm{H},{ }^{13} \mathrm{C}, \mathrm{HSQC}$ and COSY NMR spectroscopy, correlated with respect to authentic samples.

Decomposition begins when the diamine exchanges with one amido group on $\mathbf{0 P b}$ to form $\mathbf{I P b}$, which then eliminates $\mathrm{Pb}(0)$, free diamine, $\mathrm{HN}\left(\mathrm{SiMe}_{3}\right)_{2}$ and $\mathbf{I I P b}$ by oxidation of the $\mathrm{Pb}$-amido group to an imine and deprotonation of the unchelated amine to form a chelated amido group. IIPb then undergoes a second bimolecular deprotonation to form $\mathrm{HN}\left(\mathrm{SiMe}_{3}\right)_{2}$ and $\mathbf{3 P b}$ followed by [4+1] cycloreversion to diimine and $\mathrm{Pb}(0)$. Similar backbone deprotonation by $\mathrm{HN}\left(\mathrm{SiMe}_{3}\right)_{2}$ of an $\mathrm{NHC}$ was observed previously in our group with 

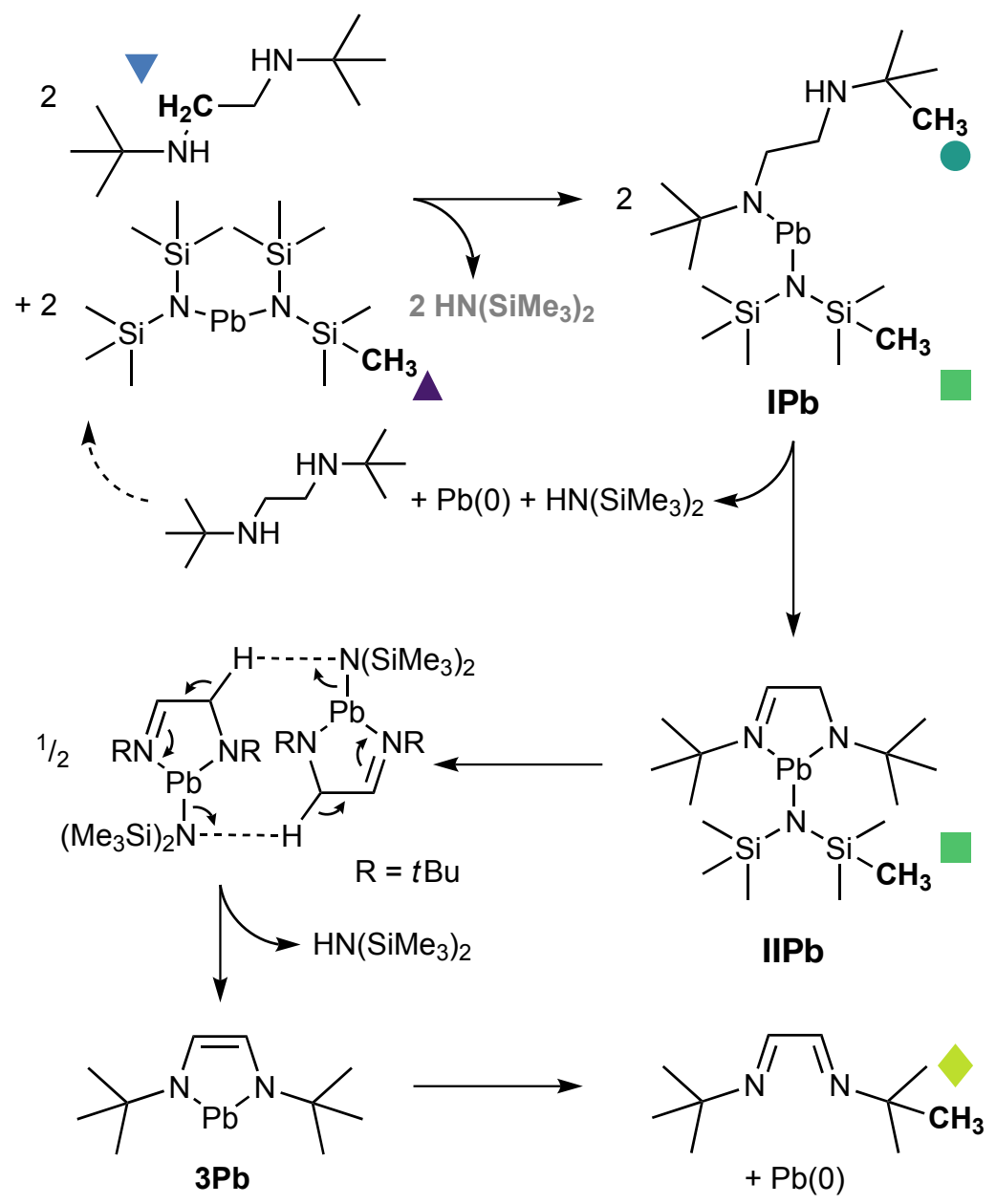

Figure 2.5: Attempted preparation of $2 \mathrm{~Pb}$ by transamination at room temperature and prosposed bimolecular dehydrogenation pathway of $N, N^{\prime}$-di-tert-butylethylene-1,2-diamine to $N, N^{\prime}$-ditert-butylethylene-1,2-diimine. In-situ ${ }^{1} \mathrm{H}$ NMR spectroscopy suggests the formation of the intermediate IPb which undergoes bimolecular redox to release free diamine, $\mathrm{HN}\left(\mathrm{SiMe}_{3}\right)_{2}$ and $\mathrm{Pb}(0)$, and generate the amido- $\alpha$-aldimino intermediate IIPb. This subsequently undergoes another deprotonation to form $3 \mathbf{P b}$ which then decomposes to diimine and $\mathrm{Pb}(0)$ by $[4+1]$ cycloreversion via the formally zero-valent diimino plumbylone. The labeled and bolded moeities correspond to the protons tracked during in-situ ${ }^{1} \mathrm{H}$ NMR spectroscopy of this reaction, shown in Figure 2.6. 


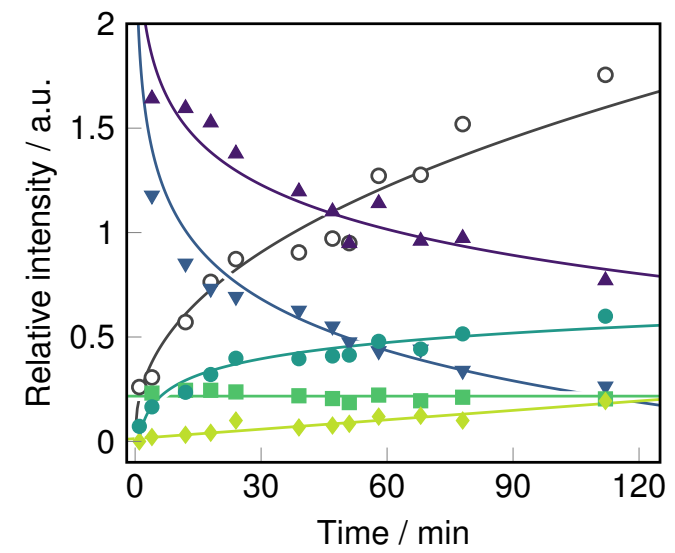

Figure 2.6: In-situ ${ }^{1} \mathrm{H}$ NMR of the first two hours of the attempted synthesis of $\mathbf{2} \mathbf{P b}$ reveals decomposition via the intermediate $\mathbf{I P b}$, which subsequently undergoes formal dehydrogenation to IIPb, the previously observed in the attempted synthesis of $\mathbf{3 P b}$ via aldimine transamination. ${ }^{121}$ Relative intensity is the integration of a respective peak calibrated with respect to residual $\mathrm{C}_{6} \mathrm{H}_{6}$, divided by the number of those protons per species (e.g. by a factor of 18 for $\left.\mathrm{HN}\left(\mathrm{SiMe}_{3}\right)_{2}\right)$. Lines and curves are aids to the eye. $\mathbf{0 P b}(\boldsymbol{\Delta})$ and $N, N^{\prime}$-di-tert-butylethylene-1,2-diamine $(\boldsymbol{\nabla})$ are consumed rapidly as $\mathrm{HN}\left(\mathrm{SiMe}_{3}\right)_{2}$ (०) is produced. Signals for tert-butyl (๑) and $-\mathrm{N}\left(\mathrm{SiMe}_{3}\right)_{2}(\square)$ protons on $\mathbf{I P b} / \mathbf{I I P b}$ suggest they quickly form an equilibrium as an intermediate resting state. Clear signals for tert-butyl groups on $N, N^{\prime}$-di-tert-butylethylene-1,2-diimine ( () grow steadily.

$\mathrm{Cu}(\mathrm{I}) \mathrm{NHC}$ complexes. ${ }^{85}$ More diimine than diamine was observed upon completion of the reaction after five (5) days, suggesting at least some free diamine substitutes $-\mathrm{N}\left(\mathrm{SiMe}_{3}\right)_{2}$ on IPb or IIPb during reaction with diamine, converting more diamine to diimine upon decomposition.

\subsubsection{Rac- $N, N^{3}$-di-tert-butylbutane-2,3-diamide lead(II) (1Pb)}

Failure to isolate $\mathbf{2} \mathbf{P b}$ and $\mathbf{3 P b}$ led us to prepare $r a c-N,{ }^{2} N^{3}$-di-tert-butylbutane-2,3-diamido lead(II) (1Pb) with a ligand that blocked the decomposition pathways observed for the other plumbylenes. The racemic butane backbone is saturated, so it does not decompose via resonance to a plumbylone like $\mathbf{3 P b}$; and the bulk of the methyl substitution sterically hinders the backbone from the dehydrogenation seen during the attempted synthesis of 2Pb. However, salt-metathesis of $\mathrm{PbCl}_{2}$ with dilithiated butanediamido salt in diethyl ether failed to produce appreciable yields of isolated $\mathbf{1 P b}$, but instead produced a black 
crystalline precipitate upon warming from $-78{ }^{\circ} \mathrm{C}$ to room temperature. Workup of the residue suggested it contained $\mathrm{LiCl}$ (dissolved in water leaving behind a black metallic precipitate), $\mathrm{Pb}(0)$ (insoluble in water, $6 \mathrm{~N} \mathrm{HCl}$; formed a white precipitate when dissolved in aqua regia; soluble in $6 \mathrm{~N} \mathrm{HNO}_{3}$ ), and an imine (NMR spectroscopy). We identified the major by-product in the filtrate by NMR spectroscopy as acetaldehyde tert-butylimine, the same imine formed when dilithio- $\alpha$-diamido salt is oxidized by dissolved $\mathrm{O}_{2}$ during quenching via $\alpha$-amido peroxy radicals. ${ }^{136}$ Possibly, stoichiometric quantities of $\mathrm{PbCl}_{2}$ substitute $\mathrm{O}_{2}$ to form organolead intermediates, which could then decompose to aldimines in a reaction similar to the Criegee oxidation of $\alpha$-diols. ${ }^{137,138}$ We were unable to synthesize $\mathbf{1 P b}$ with salt-methathesis conditions analogous to $\mathbf{1 S n}$.

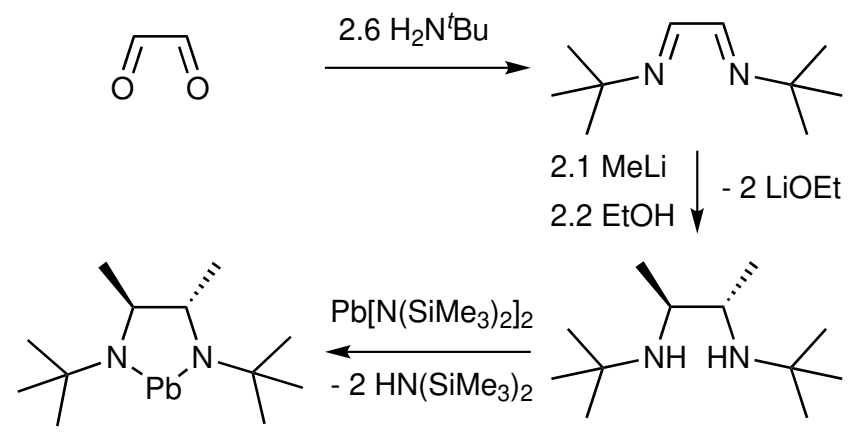

Figure 2.7: Synthesis of $\mathbf{1 P b}$ by transamination. (a) Glyoxal is reacted with ${ }^{t} \mathrm{BuNH}_{2}$ in $\mathrm{Et}_{2} \mathrm{O}$ to form di-tert-butyl-diazabutadiene (DAD), (b) then methylated twice by 2 eq. MeLi in $\mathrm{Et}_{2} \mathrm{O}$ and protonated by an excess of EtOH or $\mathrm{H}_{2} \mathrm{O}$. (c) Transamination with $\mathbf{0 P b}$ at $70{ }^{\circ} \mathrm{C}$ for 3 days yields the title compound in high yield.

The isolated yield of $\mathbf{1 P b}$ was improved to $85 \%$ by transamination of $\mathbf{0 P b}$ with one equivalent of the free rac-N, $N^{\prime}$-di-tert-butylbutane-1,2-diamine ligand in hexanes or toluene (Figure 2.7), a synthetic route that has been previously reported to prepare other NHPbs. ${ }^{122,123}$ The transamination required considerable heating and time $\left(70{ }^{\circ} \mathrm{C}, 3\right.$ days $)$ to proceed completely, likely due to the high steric demand of the diamine and leaving $\mathrm{N}\left(\mathrm{SiMe}_{3}\right)_{2}$ ligands. (Higher temperatures effect conversion in less time, but reduce yield by thermolysis of the $\mathrm{Pb}(\mathrm{II})$ species in-situ.) Removal of solvent by vacuum leaves a red oil that is a mixture of $\mathbf{1 P b}$ and free diamine, which cannot be separated by simple distil- 
lation. Pure $\mathbf{1 P b}$ is isolated from the oil as blood-red needle-like crystals (m.p. $53{ }^{\circ} \mathrm{C}$ ) by fractional sublimation under a dynamic vacuum. Briefly, free diamine was slowly removed and collected in a liquid-nitrogen cold trap with gentle warming $\left(\right.$ ca. $\left.40{ }^{\circ} \mathrm{C}\right)$ and dynamic vacuum, while any impure oil that collected on the water-cooled cold finger placed just above the crude mixture refluxed until only solid remained in the pot. After removal of excess free ligand, $\mathbf{1 P b}$ sublimed cleanly at $\geq 45^{\circ} \mathrm{C} / 10$ mTorr.

Notably, unlike acyclic diamido $\mathrm{Pb}(\mathrm{II})$ complexes, $\mathbf{1 P b}$ is not apparently thermochromic, and maintains its colour during purification and upon cooling in a $-40{ }^{\circ} \mathrm{C}$ freezer. On the other hand, $\mathbf{0 P b}$ becomes ruby red during distillation, cools to an orange solid at roomtemperature, is yellow in the same $-40^{\circ} \mathrm{C}$ freezer, and becomes colourless in liquid nitrogen. We hypothesize that the lack of thermochromicity may be due to the limited range of $\mathrm{N}-\mathrm{Pb}-\mathrm{N}$ angles available to cyclic diamido plumbylenes like $\mathbf{1 P b}\left(80 \pm 1^{\circ}\right.$ in the solidstate at $140 \mathrm{~K}$, Figure 2.8), whereas the acyclic $\mathbf{0 P b}$ is reported to change its bond angle dramatically: from $91^{\circ}$ in the gas-phase to $103.2^{\circ}$ in the solid-state at $140 \mathrm{~K} .{ }^{139}$

\subsubsection{Solid-state and solution-phase structure}

Single crystal X-ray diffraction (scXRD) crystallography on $\mathbf{1 P b}$ was used to determine its structure and degree of intermolecular interaction. The ligand was synthesized as a 1:1 racemic mixture that was not separated during purification, so the resulting $\mathrm{Pb}(\mathrm{II})$ complex was also obtained as a 1:1 racemic mixture. Both enantiomers can be seen in its asymmetric unit cell (Figure 2.8). Intermolecular interactions that are observed with less sterically demanding ligands ${ }^{122-124}$ were also sterically hindered, successfully preventing formation of the chair conformation dimer observed for saturated-backbone $\alpha$-diamido $\mathrm{NHPbs}$ with $\mathrm{SiMe}_{3}{ }^{124}$ or mesityl ${ }^{123}$ amido moeities. The stereochemically active 6 s lonepair on $\mathrm{Pb}(\mathrm{II})$ can also be inferred from the molecular structure of $\mathbf{1 P b}$, where only one $-\mathrm{CH}_{3}$ group from each tert-butylamido group faces the $\mathrm{Pb}$ center to reduce electronic interaction. 


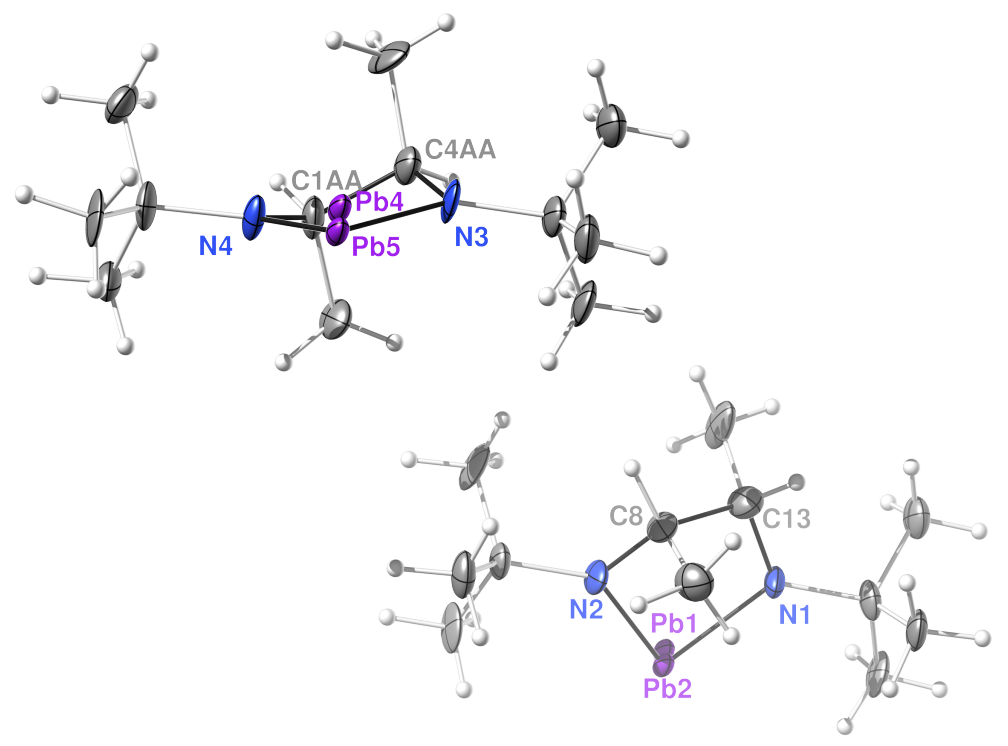

Figure 2.8: Asymmetric unit of $\mathbf{1 P b}$ in the solid-state showing both $S, S$ (left) and $R, R$ (right) enantiomers. Ellipsoids displayed at $50 \%$ probability. Selected bond lengths $(\AA)$ and angles $\left({ }^{\circ}\right)$ as averages considering the two $\mathrm{Pb}$ atoms (e.g. from $\mathrm{Pb}(1)$ and $\mathrm{Pb}(2)$ : $\mathrm{Pb}-\mathrm{N}(1)=2.150(1), \mathrm{Pb}-\mathrm{N}(2)=$ 2.121 (1); $\mathrm{N}(1)-\mathrm{Pb}-\mathrm{N}(2)=80.2(6), \mathrm{Pb}-\mathrm{N}(1)-\mathrm{C}(11)=129.24(1), \mathrm{Pb}-\mathrm{N}(2)-\mathrm{C}(12)=129.51$ (2). Disordered $\mathrm{Pb}$ atoms have the following occupancies: $\mathrm{Pb} 1$ 38.6(16)\% and $\mathrm{Pb} 2$ 61.4(16)\%; $\mathrm{Pb} 5$ 77.6(16) and $\% / \mathrm{Pb} 422.4(16) \%$.

$\mathbf{1 P b}$ packs in alternating, enantiomerically selective (i.e. $S, S$ or $R, R$ ) herringbone doublecolumns in the solid-state (Figure 2.9), with the average $\mathrm{Pb}-\mathrm{Pb}^{*}$ distance being $4.154(8) \AA$ between the disordered $\mathrm{Pb}$ atoms. A repeating long-range interaction appears to arise from $\sigma$-donation from the basic $6 \mathrm{~s}^{2}$ lone-pair on one $\mathrm{Pb}(\mathrm{II})$ atom to the empty acidic $6 \mathrm{p}_{z}^{0}$ orbital on another in an opposite column. The $\mathrm{NNPb}-\mathrm{Pb}^{*}$ angle is $91.2^{\circ}$, which corresponds very closely with calculated $6 \mathrm{~s}^{2}$ Lewis acid bonding angle of $90^{\circ}$ for $\mathrm{NNPb}-\mathrm{M}(\mathrm{M}=\mathrm{Pt}, \mathrm{Pd})$ complexes. ${ }^{140}$ Additionally, the obtuse $\mathrm{NNPb}-\mathrm{Pb}^{*}$ angle suggests that there is no $\mathrm{NN}-\mathrm{Pb}^{*}$ $\pi$-interaction. This $\mathrm{Pb}-\mathrm{Pb}^{*}$ interaction is longer than the distances reported for discrete dimers and benzannulated NHPbs (ca. 3.2-3.5 $\AA$ ), ${ }^{122,124}$ but shorter than the monomeric $\alpha$ and $\beta$-diamido NHPbs (ca. 4.2-4.6 $\AA$ ). ${ }^{123}$ The strictly monomeric plumbylene $\mathbf{0 P b}$ shows no such interaction and displays a much longer $\mathrm{Pb}-\mathrm{Pb}^{*}$ distance of $8.100 \AA{ }^{139}$ The alignment of the $\mathrm{Pb}$ atoms in the herringbone structure suggest they weakly interact in the condensed phase. 
$\mathbf{1 P b}$ and the lighter congener $\mathbf{1 S n}$ are isostructural in the solid-state. ${ }^{119}$ Significant differences between them are their $\mathrm{N}-\mathrm{M}-\mathrm{N}$ bond angles and lengths, and the torsion of the heterocyclic $\mathrm{C}_{2}$-backbone. The smaller $\mathrm{N}-\mathrm{M}-\mathrm{N}$ angle in $\mathbf{1 P b}\left(80 \pm 1^{\circ}\right)$ is due to the larger ionic radius of $\mathrm{Pb}(\mathrm{II})$ compared to $\mathrm{Sn}(\mathrm{II})\left(89 \pm 1^{\circ}\right)$, but the increased backbone torsion is less trivial to explain. High steric congestion between the butane backbone and tertbutylamido groups likely causes significant torsion on the ligand, but another possible explanation is dispersion interactions between backbone methyl groups and the empty $6 \mathrm{p}_{z}$ orbital of $\mathrm{Pb}(\mathrm{II})$ or $\mathrm{Sn}(\mathrm{II})$ add a small contribution to the crystal packing. It is worth noting that other electronic effects may contribute to the change in the ring structure, and the structural or electronic origins of the resistance to oligomerization of $\mathbf{1 S i}$ compared to 2 Si remain unknown. ${ }^{129}$

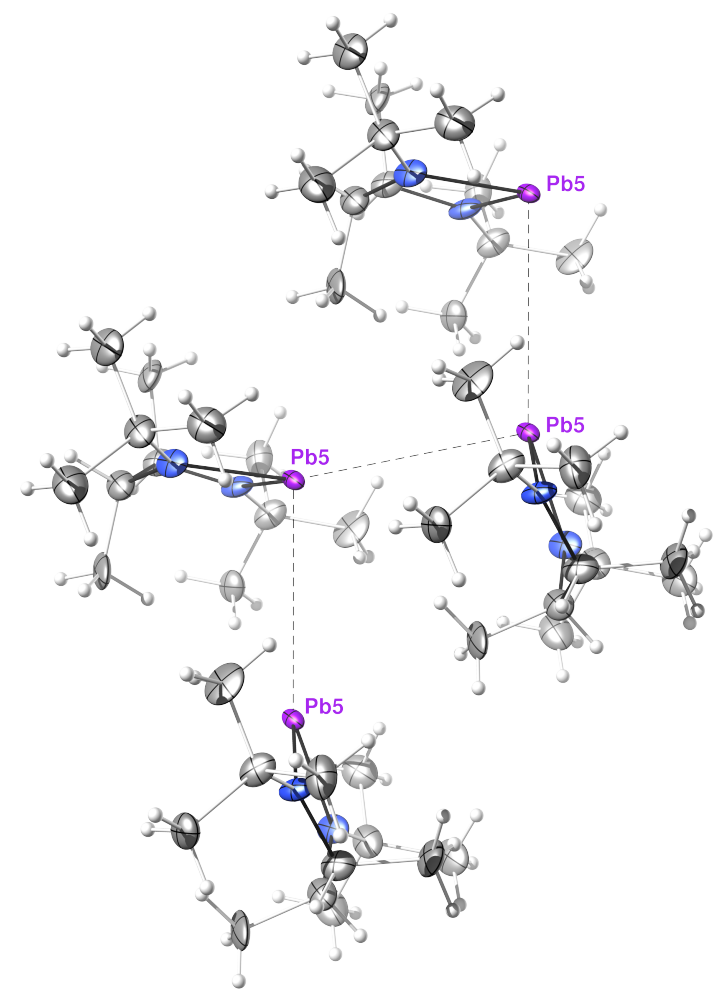

Figure 2.9: $1 \mathrm{~Pb}$ packs in alternating enantiomerically-selective herringbone double columns. An $S, S$ column (side-on, left) has $R, R$ columns (omitted) adjacent in the crystal structure. Long-range repeating $\mathrm{Pb}-\mathrm{Pb}^{*}-\mathrm{Pb}^{* *}$ interactions $(4.154(8) \AA$ ) presumably arise from Lewis acid-base stabilization via $6 \mathrm{~s}^{2} \sigma$-donation to an empty $6 \mathrm{p}_{z}^{0}$ orbital $\left(\mathrm{Pb}-\mathrm{Pb}^{*} \mathrm{NN}=91.2^{\circ}\right)$ in an opposite column). Disordered $\mathrm{Pb}$ atoms were omitted for clarity. 
High-resolution ${ }^{207} \mathrm{~Pb}$ NMR spectroscopy revealed a single peak $(\delta=+3488 \mathrm{ppm})$ that was only slightly more deshielded than the previously reported $N^{2} N^{3}$-bis-2,6-diiso-propylphenylethane-1,2-diamido lead(II) $(\delta=+3504 \mathrm{ppm}),{ }^{123}$ suggesting $\mathbf{1 P b}$ is also monomeric in solution. NHPbs' intermolecular coordination in solution can be effectively probed by ${ }^{207} \mathrm{~Pb}$ NMR spectroscopy: ${ }^{141}$ true dimers like $N,{ }^{2} N^{3}$-bis-trimethylsilylethane-1,2diamido lead(II) ${ }^{124}$ are deshielded $(\delta=+403 \mathrm{ppm})^{126}$ while NHPbs that are loosely associated dimers in solution show intermiediate shielding $(\delta \leq 3000 \mathrm{ppm}) .{ }^{127}$ Monomeric

$\mathrm{Pb}(\mathrm{II})$ diamides experience heavy shielding $(\delta=3500-5000 \mathrm{ppm}),{ }^{126}$ however it remains difficult to explain the wide range of chemical shifts among monomeric species due to the dearth of reported spectra of NHPbs in the literature, and the unknown electronic effects of endo- and exocyclic silyl functionalization. ${ }^{141}$

\subsubsection{Volatility and thermal stability of $1 \mathrm{~Pb}$}

We performed thermogravimetric analysis (TGA) of $\mathbf{1 P b}$ to assess its volatility and thermal stability, and by extension its suitability as a vapour-phase precursor (Figure 2.10). Passivation of the Pt pans with a $55 \mathrm{~nm}$ film of $\mathrm{Al}_{2} \mathrm{O}_{3}$ was necessary to prevent mixing of $\mathrm{Pb}$ and $\mathrm{Pt}$ during high-temperature experiments, as the $\mathrm{Pb} / \mathrm{Pt}$ alloy became brittle and crumbled when the pans were cleaned. Due to the kinetics of volatilization, higher initial mass loadings allow more moles of compound to be exposed to higher temperatures, allowing higher temperature decomposition to occur during the experiment and the residual mass to increase. ${ }^{142}$ Additionally, the derivative of mass with respect to temperature can indicate the onset of decomposition events as deviations from a roughly exponential curve. Together these methods provided useful estimates of the onset and extent of decomposition of $\mathbf{1 P b}$. The higher mass loadings showed that decomposition began competing with volatilization around $160{ }^{\circ} \mathrm{C}$, and the residual mass increased from $1.2 \%$ to $4.0 \%$. $1 \mathbf{P b}$ loses $1 \%$ of its initial mass at about $77{ }^{\circ} \mathrm{C}$, and a small residual mass of $1.2 \%$ was left after being held at this temperature under flow-conditions. Under the same ramp 
conditions, the well known $\mathbf{0 P b}$ loses $1 \%$ of its initial mass at about $100{ }^{\circ} \mathrm{C}$ and leaves residual masses of $8.1 \%$ and $15.7 \%$ for $13 \mathrm{mg}$ and $30 \mathrm{mg}$ loadings (Figure 1.4).
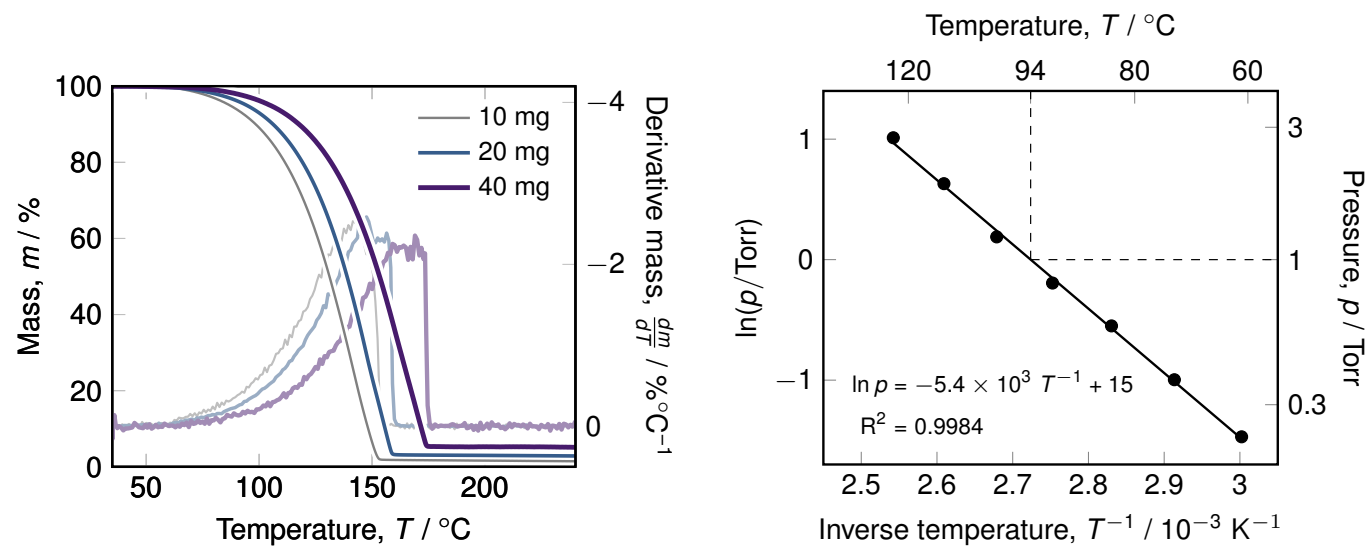

Figure 2.10: (left) TGA of $\mathbf{1 P b}$ using $10{ }^{\circ} \mathrm{C} \mathrm{min}^{-1}$ temperature ramp curves (front) and their derivatives (back) to assess thermal stability. The initial mass was increased from approximately $10 \mathrm{mg}$ (thin grey), $20 \mathrm{mg}$ (medium blue), to $40 \mathrm{mg}$ (thick purple) as a stress test on the compound, showing an increase in residual mass from $1.2 \%, 2.5 \%$, to $4.0 \%$, respectively. The emergence of secondary peaks in the derivative curves indicate the onset of decomposition at approximately $160{ }^{\circ} \mathrm{C}$, which contributes to the increase in residual mass. (right) Vapour pressure of $\mathbf{1 P b}$ modelled according to the Langmuir equation as estimated by TGA under $1 \mathrm{~atm} \mathrm{~N}_{2}$, displaying a 1 Torr vapour pressure at $94 \pm 2{ }^{\circ} \mathrm{C}$, as denoted by the dashed crosshairs.

The vapour pressure of $\mathbf{1 P b}$ was estimated by stepped isothermal TGA experiments, ${ }^{103}$ and was found to volatilize according to Equation 2.1 (Figure 2.10).

$$
\ln p=-5.4 \times 10^{3} T^{-1}+15
$$

where $p$ is the pressure in Torr, and $T$ is the temperature in $\mathrm{K}$. Accordingly, $\mathbf{1 P b}$ has a vapour pressure of 1 Torr at $94 \pm 2^{\circ} \mathrm{C}$, making it the most volatile $\mathrm{Pb}$ (II) compound reported, and among the most volatile $\mathrm{Pb}$ compounds known, behind only extremely hazardous tetraalkyl lead(IV) species like $\mathrm{Et}_{4} \mathrm{~Pb}$.

Preliminary solution-phase decomposition studies by ${ }^{1} \mathrm{H}$ and ${ }^{13} \mathrm{C}$ NMR spectroscopy showed $1 \mathrm{~Pb}$ underwent some decomposition at $90{ }^{\circ} \mathrm{C}$ in toluene- $d^{8}$ with an estimated halflife of $t_{1 / 2}=414 \mathrm{~h}$. Thermolysis products were mostly free diamine ligand, and considering the lack of unsaturated diketimine, the analogous [4+1] cycloreversion did not occur. ${ }^{118,132}$ 
It is likely trace protons were abstracted from the NMR tube's glass or through a small leak. Higher temperature decomposition in solution yielded spectra that were difficult to interpret, but the emergence of a shielded proton signal suggested the formation of an imine.

We heated a sample of neat $\mathbf{1 P b}$ under a stream of Ar below a reflux condenser and extracted the decomposed residue with benzene- $d^{6}$ to obtain better spectra with a higher concentration sample. We observed signals by ${ }^{1} \mathrm{H},{ }^{13} \mathrm{C}$, COSY and HSQC NMR spectroscopy that corresponded to acetaldehyde tert-butylimine $\left[\delta\left({ }^{1} H\right)=7.62(\mathrm{q}, 1 \mathrm{H}, \mathrm{CH}=\mathrm{N})\right.$ and $\left.\delta=1.71\left(\mathrm{~d}, 3 \mathrm{H},=\mathrm{C}(\mathrm{H}) \mathrm{CH}_{3}\right)\right],{ }^{143}$ suggesting that $\mathbf{1 P b}$ decomposes by reductive elimination of $\mathrm{Pb}(0)$ and oxidative cleavage of the backbone of the ligand as shown in Figure 2.11. The increased steric demand of the rac-butane backbone of $\mathbf{1 P b}$ effectively protects it from following the same decomposition pathway observed for $2 \mathbf{P b}$, pushing the thermal stability from below room temperature to above $150{ }^{\circ} \mathrm{C}$. We propose unimolecular $[2+2+1]$ cycloreversion as a probable thermolysis pathway for $\mathbf{1 P b}$.

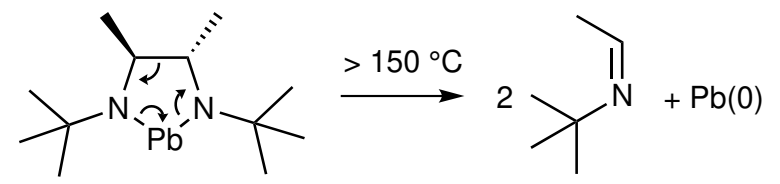

Figure 2.11: Proposed [2+2+1] cycloreversion thermolysis of $\mathbf{1 P b}$ at $150{ }^{\circ} \mathrm{C}$.

A mechanistic understanding of thermolysis is important for the development of robust ligand design and vapour deposition process, and the development of indefinitely robust precursors beyond $\mathbf{1 P b}$ would be attractive to industry for production settings, especially considering the successful development of $\mathbf{1 G e}$ and $\mathbf{1 S n} .{ }^{119,120}$ Satisfying these conditions with novel diamido ligands is the subject of an ongoing investigation. Decomposition at vaporization temperatures (i.e. ca. $90{ }^{\circ} \mathrm{C}$ ) is negligible, and its ease of synthesis makes $\mathbf{1 P b}$ an attractive potential precursor. 


\subsection{Conclusion}

$\mathrm{Pb}$ (II) was shown to oxidize $N, N^{\prime}$-di-tert-butyl- $N, N^{\prime}$-dilithiodiamidoethylene and $N, N^{\prime}$ di-tert-butyl-1-aldimino-2-amine, and also oxidize a fraction of $N, N^{\prime}$-di-tert-butylethylene1,2-diamine, cleanly to $N, N^{\prime}$-di-tert-butylethylene-1,2-diimine. Steric hindrance of the $\mathrm{C}_{2}$-bridge backbone with methyl groups blocks oxidation of the ligand and improves the stability of the heterocyclic ring system from below room temperature to above $150{ }^{\circ} \mathrm{C}$. With exceptional volatility $\left(1\right.$ Torr at $94 \pm 2^{\circ} \mathrm{C}$ ) and thermal stability $\left(\right.$ ca. $\left.150{ }^{\circ} \mathrm{C}\right), \mathbf{1 P b}$ opens a window of over $60{ }^{\circ} \mathrm{C}$ between sufficient vapour pressure for delivery and the onset of thermal decomposition. It is a promising new CVD or ALD precursor for lead-containing materials, and stands in stark contrast to its ethane- and ethylene-backboned analogues.

\subsection{Experimental Details}

\subsubsection{Synthesis}

\subsubsection{General Considerations}

All manipulations were performed under air-free conditions using standard Schlenk techniques or in a $\mathrm{N}_{2}$-filled (99.998\% purity) MBraun Labmaster 130 dry-box unless otherwise specified. Glyoxal (40 wt.\% in water), tert-butylamine (98\%), MeLi (1.6 $\left.\mathrm{M}_{\text {in }} \mathrm{Et}_{2} \mathrm{O}\right)$, ${ }^{n} \mathrm{BuLi}$ (11.0 $\mathrm{M}$ in hexanes), hexamethyldisilazane $\left[\mathrm{HN}\left(\mathrm{SiMe}_{3}\right)_{2}\right](99 \%)$, and $N, N^{\prime}$-di-tertbutylethane-2,3-diamine (98\%) $\left[\delta_{1_{\mathrm{H}}} \mathrm{NMR}\left(300 \mathrm{MHz}, \mathrm{C}_{6} \mathrm{D}_{6}, \delta_{\mathrm{C}_{6} \mathrm{H}_{6}}=7.16 \mathrm{ppm}\right): 1.05(18 \mathrm{H}\right.$, $\left.\left.s, \mathrm{C}\left(\mathrm{CH}_{3}\right)_{3}\right), 2.58\left(4 \mathrm{H}, q, \mathrm{NCH}_{2}\right), 0.7655(4 \mathrm{H}, q, \mathrm{NH})\right) . \delta_{{ }^{13} \mathrm{C}} \mathrm{NMR}\left(300 \mathrm{MHz}, \mathrm{C}_{6} \mathrm{D}_{6}, \delta_{\mathrm{C}_{6} \mathrm{H}_{6}}=\right.$

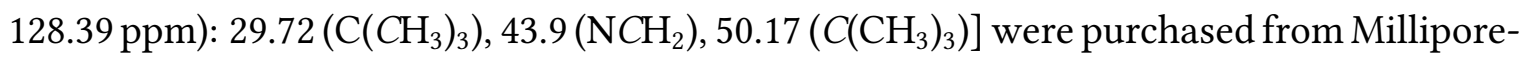
Sigma and used as received. Diethyl ether, toluene, hexanes, and pentane were ACS Reagent grade, purified by an MBraun Solvent Purifier System, and stored over $4 \AA$ molecular sieves for more than a day before use. Distilled water was degassed by sparging for 
20 min. $\mathrm{PbCl}_{2}$ was prepared by the addition of $\mathrm{HCl}(\mathrm{aq})(\mathrm{ca} .6 \mathrm{~N})$ to an ice-cooled solution of $\mathrm{Pb}\left(\mathrm{CH}_{3} \mathrm{CO}_{2}\right)_{2}$ (aq) (Baker, 95\%) until precipitation ceased. The white precipitate was collected by filtration, dried under vacuum with heating $\left(130^{\circ} \mathrm{C} / 10 \mathrm{mTorr}\right)$, and stored in a dry box. Attempted preparations of $\mathbf{3 P b}$ by salt-metathesis and transamination were reproduced according to the literature. ${ }^{121}$

\subsubsection{N,N'-di-tert-ethylene-2,3-diimine (DAD)}

$N, N^{\prime}$-di-tert-butylethylene-1,2-diimine was prepared according to a modified literature

procedure. ${ }^{119} 2.4$ eq. of neat tert-butylamine $(150 \mathrm{~mL}, 1.44 \mathrm{~mol})$ was added dropwise to a stirred $40 \mathrm{wt} . \%$ aqueous glyoxal $(69 \mathrm{~mL}, 0.60 \mathrm{~mol})$ in a round bottom flask on an ice bath under air. Shortly after addition began, DAD precipitated as a fluffy white crystalline solid. The heterogeneous suspension seized and resisted magnetic stirring as the full amount of tert-butylamine was added, so it was left overnight for the reaction to proceed completely. The next day the reaction yielded a yellow-brown clear aqueous phase and a white precipitate. The precipitate was filtered under suction and washed with distilled water to yield an off-white crystalline powder. This solid was dissolved in a minimum of anhydrous $\mathrm{Et}_{2} \mathrm{O}$, dried over anhydrous $\mathrm{MgSO}_{4}$, filtered through a medium porosity glass frit, and recrystallized at $-20^{\circ} \mathrm{C}$ overnight to yield colourless prisms of $N, N^{\prime}$-di-tertbutylethylene-1,2-diimine (65 g, 64.5\% based on starting glyoxal). $\delta_{{ }^{1} \mathrm{H}} \mathrm{NMR}(300 \mathrm{MHz}$, $\left.\left.\mathrm{C}_{6} \mathrm{D}_{6}, \delta_{\mathrm{C}_{6} \mathrm{H}_{6}}=7.16 \mathrm{ppm}\right): 1.12\left(18 \mathrm{H}, s, \mathrm{C}\left(\mathrm{CH}_{3}\right)_{3}\right), 8.07(2 \mathrm{H}, q, \mathrm{~N}=\mathrm{CH})\right) . \delta_{{ }^{13} \mathrm{C}} \mathrm{NMR}(300 \mathrm{MHz}$, $\left.\mathrm{C}_{6} \mathrm{D}_{6}, \delta_{\mathrm{C}_{6} \mathrm{H}_{6}}=128.39 \mathrm{ppm}\right): 29.71\left(s, \mathrm{C}\left(\mathrm{CH}_{3}\right)_{3}\right), 58.20\left(s, C\left(\mathrm{CH}_{3}\right)_{3}\right), 157.92(s, \mathrm{~N}=\mathrm{CH})$.

\subsubsection{Rac-N,N'-di-tert-butylbutane-2,3-diamine}

A dried $250 \mathrm{~mL}$ Schlenk flask was charged with 2.1 eq MeLi (100 mL, 1.6 M, $160 \mathrm{mmol})$ in a $\mathrm{N}_{2}$-filled dry-box. In another flask, $N, N^{\prime}$-di-tert-butylethylene-1,2-diimine (12.832 g, $76.3 \mathrm{mmol}$ ) was dissolved in about $50 \mathrm{~mL}$ of $\mathrm{Et}_{2} \mathrm{O}$, transfered to an addition funnel, and added with stirring to the MeLi at room temperature slowly enough to prevent boil- 
ing (ca. $2 \mathrm{~h}$ ). After stirring for $3 \mathrm{~h}$, the addition funnel was removed, the flask contained a colourless cloudly suspension, andd was closed with a rubber septum then removed from the dry-box. The solution was cooled on an ice bath and the reaction was quenched with an excess of degassed $\mathrm{H}_{2} \mathrm{O}$ (ca. $10 \mathrm{~mL}$ ). After stirring for an additional 30 minutes, the ethereal layer was isolated with a separation funnel, washed with water $(50 \mathrm{~mL})$, brine $(50 \mathrm{~mL})$, and dried over anhydrous $\mathrm{MgSO}_{4}$. Solvent was at atmospheric pressure on a rotary-evaporator $\left(10{ }^{\circ} \mathrm{C}\right.$ condenser, $60{ }^{\circ} \mathrm{C}$ bath $)$, and the remaining oil distilled (b.p. ca. $90{ }^{\circ} \mathrm{C} / 10$ Torr) to afford $13.464 \mathrm{~g}(67.2 \mathrm{mmol})$ of free diamine as a colourless liquid (88\% based on starting diimine). ${ }^{1} \mathrm{H}$ NMR data agreed with literature values. ${ }^{119,144}$ $\delta_{{ }^{1} \mathrm{H}} \mathrm{NMR}\left(300 \mathrm{MHz}, \mathrm{CDCl}_{3}, \delta_{\mathrm{Si}\left(\mathrm{CH}_{3}\right)_{4}}=0 \mathrm{ppm}\right): 0.96(2 \mathrm{H}, b r, \mathrm{NH}), 1.04\left(6 \mathrm{H}, d, \mathrm{C}(\mathrm{H}) \mathrm{CH}_{3}\right)$, $1.08\left(18 \mathrm{H}, s, \mathrm{C}\left(\mathrm{CH}_{3}\right)_{3}\right), 2.40\left(2 \mathrm{H}, q, \mathrm{C}(\mathrm{H}) \mathrm{CH}_{3}\right)$.

\subsubsection{Bis[bis(trimethylsilyl)amido] lead(II) (0Pb)}

Lithium bis(trimethylsilyl)amide $\left[\mathrm{LiN}\left(\mathrm{SiMe}_{3}\right)_{2}\right]$ was prepared according to a previously detailed method from ${ }^{n} \mathrm{BuLi}(100 \mathrm{~mL}, 11.0 \mathrm{M}$ in hexanes, $1.1 \mathrm{~mol})$ and hexamethyldisilazane (195 g, $1.2 \mathrm{~mol})$ in ca. $1 \mathrm{~L}$ of hexanes at room temperature. Distillation through a $90^{\circ}$ elbow $\left(70{ }^{\circ} \mathrm{C} / 10 \mathrm{mTorr}\right)$ afforded $169.2 \mathrm{~g}$ (1.01 mol) of colourless crystals of $\mathrm{LiN}\left(\mathrm{SiMe}_{3}\right)_{2}$ (92\%, based on $\left.{ }^{n} \mathrm{BuLi}\right) .{ }^{145}$

$\mathbf{0 P b}$ was prepared according to a modified literature procedure. ${ }^{146}$ Typically, in a $\mathrm{N}_{2}$ filled dry-box, a $500 \mathrm{~mL}$ round-bottom flask was charged with 1.1 eq. $\mathrm{PbCl}_{2}(14.49 \mathrm{~g}$, $52 \mathrm{mmol})$ and 2 eq. $\mathrm{LiN}\left(\mathrm{SiMe}_{3}\right)_{2}(22.89 \mathrm{~g}, 95 \mathrm{mmol}), 200 \mathrm{~mL}$ hexanes and 2 eq. $\mathrm{Et}_{2} \mathrm{O}(7.0 \mathrm{~g}$, $95 \mathrm{mmol}$ ) was added all at once, the flask was sealed with a greased glass stopper, and clipped shut to prevent escape of solvent during the exothermic salt-metathesis. The etherate $\mathrm{LiN}\left(\mathrm{SiMe}_{3}\right)_{2} \cdot\left(\mathrm{Et}_{2} \mathrm{O}\right)^{147}$ can be subsituted and the added diethyl ether omitted if distilled $\mathrm{LiN}\left(\mathrm{SiMe}_{3}\right)_{2}$ is unavailable. The rate of reaction was conveniently limited by the

relative insolubility of $\mathrm{PbCl}_{2}$ in hydrocarbon solvents, and the absense of $\mathrm{Et}_{2} \mathrm{O}$ led to very little conversion (ca. 5\%). However, the use of $\mathrm{Et}_{2} \mathrm{O}$ as the solvent consistently decreased 
final yields to $70 \%$ or less. The reaction was stirred overnight to reach completion.

The next day, the flask contained a canary-yellow coloured supernatant liquid with an off-white precipitate. Precipitated $\mathrm{LiCl}$ and excess $\mathrm{PbCl}_{2}$ were removed by filtration over Celite, and solvent and free $\mathrm{HN}\left(\mathrm{SiMe}_{3}\right)_{2}$ were removed under reduced pressure at ambient temperature to afford $\mathbf{0 P b}$ contaminated with $\mathrm{HN}\left(\mathrm{SiMe}_{3}\right)_{2}$ and $\mathrm{LiN}\left(\mathrm{SiMe}_{3}\right)_{2}$ as an orange-red oil. This mixture is not stable at room-temperature in the dark under inert atmosphere and purification by distillation is required for storage. Vacuum distillation $\left(90^{\circ} \mathrm{C} / 10 \mathrm{mTorr}\right.$ ) over a small amount of $\mathrm{PbCl}_{2}$ (to consume remaining $\mathrm{LiN}\left(\mathrm{SiMe}_{3}\right)_{2}$ ) yields $\mathbf{0 P b}\left(23.8 \mathrm{~g}, 95 \%\right.$ based on $\left.\operatorname{LiN}\left(\mathrm{SiMe}_{3}\right)_{2}\right)$ as a thermochromic low-melting point solid that is ruby-red at $90{ }^{\circ} \mathrm{C}$, orange at room temperature, yellow at $-78{ }^{\circ} \mathrm{C}$, and colourless at $-196{ }^{\circ} \mathrm{C} . \delta_{1 \mathrm{H}} \mathrm{NMR}\left(300 \mathrm{MHz}, \mathrm{C}_{6} \mathrm{D}_{6}, \delta_{\mathrm{Si}\left(\mathrm{CH}_{3}\right)_{4}}=0 \mathrm{ppm}\right): 0.96\left(36 \mathrm{H}, s, \mathrm{Si}\left(\mathrm{CH}_{3}\right)_{3}\right)$. We found that filtration was also not necessary, and the product can be distilled directly from the crude pasty mixture after removal of volatiles by vacuum at room temperature. It is worth noting that this procedure works equally well with $\mathrm{SnCl}_{2}$ to yield the lighter congener and useful synthon $\mathrm{Sn}\left[\mathrm{N}\left(\mathrm{SiMe}_{3}\right)_{2}\right]_{2}$.

\subsubsection{Attempted synthesis of rac- $N,{ }^{2} N^{3}$-di-tert-butylbutane-2,3-diamido lead(II) (1Pb) via salt metathesis of $\mathrm{PbCl}_{2}$}

The dilithio diamidobutane salt was prepared as for the free diamine from DAD ( $3 \mathrm{~g}$, $17.8 \mathrm{mmol}$ ) and MeLi (36.6 mmol), but was used in-situ as reported for 1Ge and 1Sn. ${ }^{119}$ It was added with a dropping funnel over $2.5 \mathrm{~h}$ to a stirred slurry of $\mathrm{PbCl}_{2}$ in $\mathrm{Et}_{2} \mathrm{O}$ cooled to $-78{ }^{\circ} \mathrm{C}$ in a Schlenk flask shielded from light. A red solution and black precipitate form immediately. After addition, the slurry was stirred overnight. Cooling was not sustained and the solution warmed slowly to room-temperature. A red supernatant liquid with black crystalline precipitate remained. All volatiles were removed under vacuum, leaving a grey residue. A small amount of a red oil, determined to be highly impure $\mathbf{1 P b}$ (ca. $\leq 5 \%$, based on $\left.\mathrm{PbCl}_{2}\right)$ by NMR spectroscopy, was vacuum distilled $\left(70{ }^{\circ} \mathrm{C} / 10 \mathrm{mTorr}\right)$ from the residue. 
${ }^{1} \mathrm{H}$ NMR spectroscopy on a filtered benzene- $d^{6}$ extract of the residue revealed, among starting material, peaks corresponding to acetaldehyde tert-butylimine $\delta\left({ }^{1} H\right)=7.62$ (q, $1 \mathrm{H}, \mathrm{CH}=\mathrm{N})$ and $\delta=1.71\left(\mathrm{~d}, 3 \mathrm{H},=\mathrm{C}(\mathrm{H}) \mathrm{CH}_{3}\right) .{ }^{143}$ This imine was also recovered from the cold-trap of the vacuum line used to remove solvent from the reaction mixture.

\subsubsection{Successful preparation of $1 \mathrm{~Pb}$ via transamination}

In a $\mathrm{N}_{2}$ filled glove box, 1 eq. rac- $N, N^{\prime}$-di-tert-butylbutane-1,2-diamine (0.92 g, $\left.4.6 \mathrm{mmol}\right)$ is added to 1 eq. $\mathbf{0 P b}(2.41 \mathrm{~g}, 4.6 \mathrm{mmol})$ in $75 \mathrm{~mL}$ hexanes or toluene in an O-ring sealed, PTFE-capped, heavy-walled $150 \mathrm{~mL}$ pressure vessel. The mixture is heated to $70{ }^{\circ} \mathrm{C}$ for 3 days to completely convert $\mathbf{0 P b}$ to $\mathbf{1 P b}$ as indicated by ${ }^{1} \mathrm{H}$ NMR. Less time will lead to incomplete conversion, higher temperatures lead to a decrease in yield due to decomposition, and we were unable to effectively separate $\mathbf{0 P b}$ from $\mathbf{1 P b}$ by recrystallization or distillation. Slight decomposition is signaled by the precipitation of a black crystalline material-presumably $\mathrm{Pb}(0)$. The dark-red suspension is cooled to room-temperature and brought into a dry-box where it is filtered over Celite to remove the black precipitate, yielding a blood-red solution. Free $\mathrm{HN}\left(\mathrm{SiMe}_{3}\right)_{2}$ and solvent are removed under vacuum, and the impure residue is purified by fractional vacuum sublimation onto a water-cooled cold-finger $\left(60{ }^{\circ} \mathrm{C} / 10 \mathrm{mTorr}\right)$ to afford $\mathbf{1 P b}(1.66 \mathrm{~g}, 89 \%$ based on $\mathbf{0 P b})$ as an extremely airand moisture-sensitive blood-red crystalline solid. Samples slowly darken upon standing in light under inert atmosphere after $>2$ weeks. M.p. $53{ }^{\circ} \mathrm{C}$, sublimed. Found: C, 35.0; $\mathrm{H}, 6.3 ; \mathrm{N}, 7.1 . \mathrm{C}_{12} \mathrm{H}_{26} \mathrm{~N}_{2} \mathrm{~Pb}$ requires: $\mathrm{C}, 35.5 ; \mathrm{H}, 6.5 ; \mathrm{N}, 6.9 \%$. HRMS (EI) Found, $m / z=$ 406.18570, calc. 406.1864 , dev. $-0.54 \mathrm{mmu} . \delta_{{ }^{1} \mathrm{H}} \mathrm{NMR}\left(300 \mathrm{MHz}, \mathrm{C}_{6} \mathrm{D}_{6}, \delta_{\mathrm{C}_{6} \mathrm{H}_{6}}=7.16 \mathrm{ppm}\right)$ : $\left.1.35\left(18 \mathrm{H}, s, \mathrm{C}\left(\mathrm{CH}_{3}\right)_{3}\right), 1.45\left(6 \mathrm{H}, d, \mathrm{C}(\mathrm{H}) \mathrm{CH}_{3}\right), 5.37\left(2 \mathrm{H}, q, \mathrm{C}(H) \mathrm{CH}_{3}\right)\right) . \delta_{{ }^{13} \mathrm{C}} \mathrm{NMR}(300 \mathrm{MHz}$ $\left.\mathrm{C}_{6} \mathrm{D}_{6}, \delta_{\mathrm{C}_{6} \mathrm{H}_{6}}=128.06 \mathrm{ppm}\right): 30.53\left(\mathrm{C}(\mathrm{H}) \mathrm{CH}_{3}\right), 35.07\left(\mathrm{C}\left(\mathrm{CH}_{3}\right)_{3}\right), 59.42\left(C\left(\mathrm{CH}_{3}\right)_{3}\right), 73.61\left(\mathrm{C}(\mathrm{H}) \mathrm{CH}_{3}\right)$. 


\subsubsection{Characterization}

\subsubsection{In-situ NMR spectroscopy of the attempted preparation of $2 \mathrm{~Pb}$}

In a drybox, $\mathbf{0 P b}(0.143 \mathrm{~g}, 0.27 \mathrm{mmol})$ was dissolved in dry, degassed $\mathrm{C}_{6} \mathrm{D}_{6}(\mathrm{ca} .1 \mathrm{~mL})$ in an NMR tube, closed with a plastic cap, and sealed with Parafilm. $N, N^{\prime}$-di-tert-butylethylene1,2-diamine $(0.048 \mathrm{~g}, 0.28 \mathrm{mmol})$ was also dissolved in $\mathrm{C}_{6} \mathrm{D}_{6}(\mathrm{ca} .0 .5 \mathrm{~mL})$ in a small vial and capped with a rubber septum. A spectrum was obtained of $\mathbf{0 P b}$, then the tube was removed from the spectrometer and the diamine solution was injected with a syringe through the cap all at once. The NMR tube was resealed with more Parafilm, shaken vigorously for approximately $30 \mathrm{~s}$, and placed back in the spectrometer. The sample was locked and shimmed before each acquisition, when 16 scans were acquired.

\subsubsection{Thermogravimetric analysis (TGA)}

TGA was performed with a TA Instruments Q50 kept in an MBraun Labmaster 130 drybox filled with $\mathrm{N}_{2}$. Pt pans were coated before each experiment with approximately $55 \mathrm{~nm}$ of $\mathrm{Al}_{2} \mathrm{O}_{3}$ by atomic layer deposition (ALD) in a Picosun $\mathrm{R}-150$ reactor using 500 cycles of trimethylaluminum (TMA) and water at $200{ }^{\circ} \mathrm{C}$ with $0.1 \mathrm{~s}$ pulses and $8 \mathrm{~s}$ purges each. This is critical to protect the Pt pans as they become brittle and break easily after hightemperature exposure to $\mathrm{Pb}(0)$ residues from precursor decomposition. The TGA furnace was purged with $99.999 \% \mathrm{~N}_{2}$ at $60 \mathrm{sccm}$ and the temperature was increased at $10{ }^{\circ} \mathrm{C} \mathrm{min}-1$ for the "ramp" experiments, and increased at $40{ }^{\circ} \mathrm{C} \mathrm{min}^{-1}$ to increase temperatures stepwise to hold at isotherms of $60-130{ }^{\circ} \mathrm{C}$ for $7 \mathrm{~min}$ each for the "stepped isothermal" experiments. The exact masses for the $10 \mathrm{mg}, 20 \mathrm{mg}$, and $40 \mathrm{mg}$ loadings shown in Figure 2.10 were $11.61886 \mathrm{mg}, 20.40275 \mathrm{mg}$, and $39.35869 \mathrm{mg}$, respectively. 


\subsubsection{In-situ NMR spectroscopy of thermolysis of $1 \mathrm{~Pb}$}

${ }^{1} \mathrm{H}$ and ${ }^{13} \mathrm{C}$ NMR spectra were obtained after heating a $15 \mathrm{mM}$ solution of $\mathbf{1 P b}$ in toluene$d^{8}$ in a sealed heavy-walled glass tube at $90^{\circ} \mathrm{C}$ for 7 days. Spectra were collected, every $12 \mathrm{~h}$ after cooling the sample to room-temperature. At $90{ }^{\circ} \mathrm{C}$, formation of a black precipitate and signals corresponding to the free ligand $\mathrm{L}(\mathrm{NH})_{2}$ (see above) appeared. This same sample was subjected to $6 \mathrm{~h}$ at $150{ }^{\circ} \mathrm{C}$ as before, and spectra were obtained after cooling. Low-resolution spectra were obtained due to poor shimming from suspended particles, but a signal at high chemical shift ( $\delta \sim 7.6 \mathrm{ppm})$ was observed by ${ }^{1} \mathrm{H}$ NMR spectroscopy that prompted another decomposition study.

\subsubsection{Neat thermolysis of $1 \mathrm{~Pb}$}

$\mathbf{1 P b}(0.302 \mathrm{~g}, 0.74 \mathrm{mmol})$ was placed in a $50 \mathrm{~mL}$ Schlenk flask in a dry-box and placed under a strong over-pressure of ultra-pure Ar (99.999\%) on a Schlenk line. It was then joined to a previously flame-dried reflux condenser fitted with a gas inlet at the top, and heated to $150{ }^{\circ} \mathrm{C}$ with an oil bath for $6 \mathrm{~h}$. After cooling, the black residue was extracted with anhydrous (stored over $4 \AA$ sieves), degassed benzene- $d^{6}$ and transfered to a dried NMR tube under a stream of ultra-pure $\mathrm{N}_{2}(99.999 \%)$. The dark-brown, murky sample was analyzed immediately by ${ }^{1} \mathrm{H}$ and COSY NMR spectroscopy, and a ${ }^{13} \mathrm{C}$ NMR spectrum (14 336 scans) was obtained overnight. Another ${ }^{1} \mathrm{H}$ spectrum was obtained the following day, which showed essentially the same spectrum with better resolution since the suspended $\mathrm{Pb}(0)$ solids had settled. Clear signals corresponding to an imino hydrogen $\left[\delta\left({ }^{1} H\right)=7.62\right.$ $(\mathrm{q}, 1 \mathrm{H}, \mathrm{CH}=\mathrm{N})]$ that correlated $(\mathrm{COSY})$ to another signal $\left[\delta=1.71\left(\mathrm{~d}, 3 \mathrm{H},=\mathrm{C}(\mathrm{H}) \mathrm{CH}_{3}\right)\right]$ presumably belonging to acetal hydrogens. The tert-butyl region was obscured by signals from free diamine ligand and surviving $\mathbf{1 P b}$; the origin of the protonated diamine remains unclear. The emergent signals correspond to the reported chemical shifts of acetaldehyde tert-butylimine. ${ }^{143}$ Which enantiomers were formed was out of the scope of this study. 


\subsubsection{Single-crystal X-ray diffraction of $1 \mathrm{~Pb}$}

$\mathbf{1 P b}$ is highly soluble in polar and apolar non-protic solvents such as diethyl ether, tetrahydrofuran, n-hexane, n-pentane and toluene. Crystals suitable for single-crystal X-ray difraction (scXRD) were grown by recrystallization of in a 1:1 mixture of diethyl ether and toluene at $-30{ }^{\circ} \mathrm{C}$, which were then coated with Paratone- $\mathrm{N}$ oil, mounted on a fiber loop, and placed in a cold, gaseous $\mathrm{N}_{2}$ stream on a Rigaku-Oxford SuperNova diffractometer. Diffraction was performed using $\omega$ scans at $100 \mathrm{~K}$ and intensities were measured using graphite monochromatic $\mathrm{Cu} \mathrm{K} \alpha$ radiation $(\lambda=1.54184 \AA)$. Data collection, indexing, initial cell refinements, frame integration, final cell refinements, and absorption corrections were accomplished with Agilent CrysAlis Pro v1.171.37.34. Space groups were assigned by analysis of the metric symmetry and systematic absences (determined by WinGX). The structure was solved (in a manner similar to the congener $\mathbf{1 S n}{ }^{119}$ ) with ShelxT and refined against all data in the reported $2 \theta$ ranges by full-matrix least-squares on $\mathrm{F}^{2}$ with the SHELXL program suite using the ShelxLe interface. ${ }^{148}$ The twin-law was determined using PLATON TwinRotMat ${ }^{149}$ and further refined as a two-component twin with a ratio of 0.574 and 0.426 , respectively. EADP was used in the refinement of the $\mathrm{Pb}$ atoms. The disordered $\mathrm{Pb}$ atoms displayed site occupancies of 0.39:0.61 for $\mathrm{Pb} 1: \mathrm{Pb} 2$ and 0.22:0.78 for

$\mathrm{Pb} 4: \mathrm{Pb} 5$. Images were rendered with UCSF Chimera. ${ }^{150}$ The data was deposited in the Cambridge Structural Database (CSD) with indentification number: CCDC 1587313.

\subsubsection{Powder X-ray diffraction}

Bulk purity was assessed by powder X-ray diffraction (pXRD) and compared to the simulated spectrum as calculated based on the single-crystal structure determined previously, see Supplementary Information. A theoretical diffraction pattern of $\mathbf{1 P b}$ was calculated based on the structural data set obtained by scXRD (see above) by Crystal Impact DIAMOND v3.2. The simulation was carried out for the $2 \theta$ angle range of $5^{\circ}$ to $60^{\circ}$ from irradiation of a $\mathrm{Cu} \mathrm{K} \alpha \mathrm{X}$-Ray source. $50 \mathrm{mg}$ of $\mathbf{1 P b}$ were ground in a pestle-and-mortar in 
a dry-box, then carefully filled in a glass capillary (Mark capillary for X-Ray microstructure analysis). The capillary was sealed with grease, flame sealed, mounted on a rotatable sample holder and geometrically aligned to avoid static imbalance during the measurement. The pXRD experiments was conducted with a Bruker D8 Advance diffractometer [X-Ray source $\mathrm{Cu} \mathrm{K} \alpha(\lambda=1.54184 \AA), 40 \mathrm{kV}, 30 \mathrm{~mA}$ ] in Bragg-Brentano geometry in a $2 \theta$ range of $5^{\circ}$ to $60^{\circ}$. Diffraction data were processed using Bruker AXS Diffrac ${ }^{\text {plus }}$ EVA v10.0.

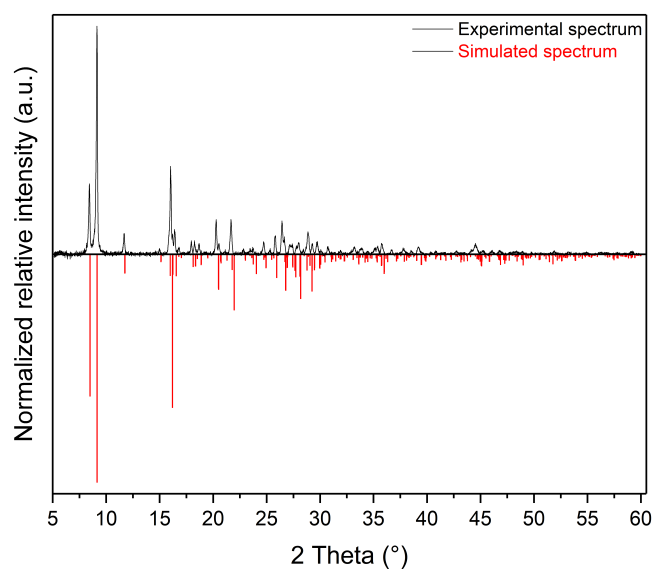

Figure 2.12: Experimental (top, black) and simulated (bottom, red) powder X-ray diffraction (pXRD) patterns for $\mathbf{1 P b}$. 


\section{Chapter 3}

\section{Ultralow Temperature Atomic Layer Deposition of PbS}

Reprinted and adapted from the original article published as:

Georgi Popov, Goran Bačić, Miika Mattinen, Toni Manner, Hannu Lindström, Heli Seppänen, Sami Suihkonen, Marko Vehkamäki, Marianna Kemell, Pasi Jalkanen, Kenichiro Mizohata, Jyrki Räisänen, Markku Leskelä, Hanna Maarit Koivula, Seán T. Barry, and Mikko Ritala. Atomic Layer Deposition of PbS Thin Films at Low Temperatures. Chem. Mater., 2020, 32, 19, 8216-8228. Copyright 2020 American Chemical Society.

Department of Chemistry, Carleton University, 1125 Colonel By Drive, Ottawa, Ontario, K1S 5B6, Canada 


\section{Abstract}

Atomic layer deposition (ALD) is a viable method for depositing functional, passivating, and encapsulating layers on top of halide perovskites. Studies in that area have only focused on metal oxides, despite a great number of materials that can be made with ALD. This work demonstrates that, in addition to oxides, other ALD processes can be compatible with the perovskites. We describe two new ALD processes for lead sulfide. These processes operate at low deposition temperatures $\left(45-155^{\circ} \mathrm{C}\right)$ that have been inaccessible to previous ALD PbS processes. Our processes rely on volatile and reactive lead precursors lead(II) bis[bis(trimethylsilyl)amide] (0Pb) and lead(II) rac- $N, N^{\prime}$-di-tert-butylbutane-2,3diamide $(\mathbf{1 P b})$ with $\mathrm{H}_{2} \mathrm{~S}$. These precursors produce high quality $\mathrm{PbS}$ thin films that are uniform, crystalline, and pure. The films exhibit p-type conductivity and good mobilities of $10-70 \mathrm{~cm}^{2} \mathrm{~V}^{-1} \mathrm{~s}^{-1}$. Ultralow-temperature deposition with $\mathbf{1 P b}$ enable direct ALD of $\mathrm{PbS}$ onto a halide perovskite $\mathrm{CH}_{3} \mathrm{NH}_{3} \mathrm{PbI}_{3}$ (MAPI) without its decomposition. The stability of MAPI in ambient air is greatly improved by capping with ALD PbS. More generally, these new processes offer valuable alternatives for PbS-based devices, and we hope that this study will inspire more studies on ALD of non-oxides on halide perovskites. 


\subsection{Introduction}

Lead(II) sulfide $(\mathrm{PbS})$ is one of the oldest known and most well-studied semiconductors. Depending on whether excess lead or sulfur is present, $\mathrm{PbS}$ exhibits either $n$-type or $p$ type semiconductivity. ${ }^{151}$ The band gap of $\mathrm{PbS}$ is narrow $(0.4 \mathrm{eV})$; therefore, $\mathrm{PbS}$ absorbs visible and near-mid-infrared light. The exciton Bohr radius in PbS is large (ca. $20 \mathrm{~nm}$ ) compared to most semiconductors which enables quantum confinement effects to manifest at relatively large dimensions, causing widening of the band gap. ${ }^{152}$ These unique properties enable application of PbS thin films in photovoltaics, ${ }^{153,154}$ photodetectors, ${ }^{155,156}$ transistors, ${ }^{157}$ and gas sensors. ${ }^{158,159} \mathrm{PbS}$ quantum dots demonstrate an even wider range of applications. ${ }^{157}$ Atomic layer deposition (ALD) is capable of producing both $\mathrm{PbS}$ thin films ${ }^{160}$ and quantum dots. ${ }^{35,161}$ Our main interest in ALD of PbS is in using PbS thin films together with halide perovskites.

Halide perovskites are a class of materials with remarkable optoelectronic properties. Perovskites have radically changed the landscape in the photovoltaics research ${ }^{162}$ and are studied in a variety of other devices such as X-ray scintillators, ${ }^{163}$ photodetectors, ${ }^{164,165}$ light-emitting diodes (LEDs), ${ }^{166}$ and transistor ${ }^{167}$ as well as memory components. ${ }^{168,169}$ However, halide perovskites also face stability and scalability issues that hinder their commercialization. Perovskites decompose in ambient air, and scalable deposition methods must be developed for all layers in perovskite-based devices for their production. One approach to tackle these issues is to use ALD for thin film deposition while making these devices. ALD is compatible with roll-to-roll processing and is inherently scalable to large batches and deposition areas. ALD offers uniform and pinhole-free films and ability to conformally coat even the most demanding 3D structures with high aspect ratios. When deposited on top of perovskite, such films encapsulate the perovskite and improve its stability in ambient air. ${ }^{170}$ Because of these characteristics, ALD has recently made its way into perovskite photovoltaics. ${ }^{171}$ Zardetto et al., ${ }^{76}$ Seo et al., ${ }^{172}$ Brinkmann et al., ${ }^{173}$ and 
Raiford et al. ${ }^{174}$ have given comprehensive reviews on this topic.

A great number of different materials can be deposited with ALD: at least 501 unique compositions according to the database of ALD processes. ${ }^{175}$ However, in context of ALD on perovskites, previous studies have focused on a few selected ALD oxides such as $\mathrm{Al}_{2} \mathrm{O}_{3}$ and $\mathrm{SnO}_{x} \cdot{ }^{76,172,173}$ This disparity is caused by the relatively high deposition temperatures $\left(100-400{ }^{\circ} \mathrm{C}\right)$ that most ALD processes require. These deposition temperatures are incompatible with the low thermal budget $\left(\right.$ ca. $\left.\leq 100{ }^{\circ} \mathrm{C}\right)$ of the perovskites. Another incompatibility issue arises from chemical reactions between the ALD precursors and the perovskites. ${ }^{176}$ In this work we show that, in addition to the handful of ALD oxide processes, also ALD sulfide processes can be compatible with the perovskites. In particular, $\mathrm{PbS}$ can be deposited directly on the $\mathrm{CH}_{3} \mathrm{NH}_{3} \mathrm{PbI}_{3}$ (MAPI) perovskite, improves the stability of the perovskite, and has valuable functional properties. We chose $\mathrm{PbS}$ because it can exhibit $p$ type semiconductivity which is scarce among perovskite compatible ALD materials and because PbS electrical as well as optical properties can be tuned. We aimed to achieve proper protective and carrier transport properties in one ALD layer that is compatible with the perovskites. Combining two functions into one ALD layer provides the advantage of simplifying the device structure and its deposition procedure. ${ }^{177}$ Very few ALD processes are capable of depositing films with such properties, and $p$-type semiconductors suitable for hole transporting layers are even more scarce. ${ }^{76,174}$ Previous studies have already demonstrated the applicability of $\mathrm{PbS}$ films in perovskite photovoltaics. ${ }^{178,179} \mathrm{We}$ believe that protective, $p$-type PbS can be used in other $p$ - $i$ - $n$ junction based devices such as LEDs, ${ }^{166}$ memory components, ${ }^{169}$ and photodetectors. ${ }^{164,165}$ Devices based on other structures can still exploit the protective property of $\mathrm{PbS}$ provided that the conductivity of the protective layer is not detrimental. PbS films also have another useful property: a series of chemical treatments convert them into MAPI films while retaining the typical advantages of ALD, as demonstrated by Sutherland et al. ${ }^{32,180}$ in one of the first studies involving both perovskites and ALD. 
Several ALD processes already exist for PbS (Figure 3.1b). ${ }^{160,181}$ These processes operate in a temperature range of $130-400{ }^{\circ} \mathrm{C}$. Such temperatures are incompatible with the halide perovskites. For example, MAPI, the most well-known halide perovskite, decomposes completely at $130{ }^{\circ} \mathrm{C}$ in our ALD reactors. Lowering the deposition temperature below $130{ }^{\circ} \mathrm{C}$ with the earlier ALD PbS processes is impossible because the lead precursors are either not sufficiently volatile or reactive at lower temperatures. Hence, we decided to look into developing new ALD processes for $\mathrm{PbS}$ that would work at lower temperatures. In our previous work on $\mathrm{ALD}$ of $\mathrm{PbI}_{2}{ }^{34}$ we had identified one lead precursor that is volatile and reactive at low temperatures: lead(II) bis[bis(trimethylsilyl)amide], $\mathbf{0 P b}$ (Figure 3.1a). Another potentially suitable precursor, also a diamide, lead(II) $\operatorname{rac}-N, N^{\prime}$-ditert-butylbutane-2,3-diamide, $\mathbf{1 P b}$ (Figure 3.1a), was recently reported by Bačić et al. ${ }^{182}$ In this work we describe two new ALD processes for $\mathrm{PbS}$ using $\mathbf{0 P b}$ and $\mathbf{1 P b}$ as lead precursors and $\mathrm{H}_{2} \mathrm{~S}$ as a sulfur precursor. We also demonstrate that these $\mathrm{PbS}$ processes are compatible with MAPI films and discuss challenges that we encountered during the scale-up of the $\mathbf{1 P b}$ synthesis.

\subsection{Results and Discussion}

\subsubsection{Precursor Characteristics, Synthesis, and Scale-Up}

$\mathbf{0 P b}$ and $\mathbf{1 P b}$ (Figure 3.1a) are diamidoplumbylenes with low melting points $\left(32\right.$ and $53{ }^{\circ} \mathrm{C}$, respectively) and high volatility $\left(60\right.$ and $40{ }^{\circ} \mathrm{C}$ precursor source temperatures in the F120 ALD reactor, respectively). While $\mathbf{0 P b}$ was readily scalable through a facile salt-metathesis reaction, $\mathbf{1 P b}$ had only been synthesized on a preparative scale by transamination of

$\mathbf{0 P b}{ }^{182}$ ALD process development required larger quantities of $\mathbf{1 P b}$, and scale-up uncovered deficiencies in the original procedure. We found that residual $\mathbf{0 P b}$ as low as $5 \%$ in the crude product mixture after the transamination lowered the melting point mixture to far below room temperature. All attempts to separate $\mathbf{1 P b}$ and $\mathbf{0 P b}$ by recrystalliza- 
tion failed, and fractional sublimation was complicated by their similar volatility. While $\mathbf{1 P b}$ sublimed first, the partial pressure of $\mathbf{0 P b}$ rose when $\mathbf{1 P b}$ was depleted in the sublimation pot; thus, $\mathbf{0 P b}$ began to sublime and mix with crystalline $\mathbf{1} \mathbf{P b}$ on the coldfinger. This caused the crystalline $\mathbf{1 P b}$ to become a molten mixture that would drip back into the pot indefinitely. Thus, all $\mathbf{0 P b}$ must either be converted to $\mathbf{1 P b}$ or destroyed by thermolysis for effective purification. Reaction monitoring by ${ }^{1} \mathrm{H}$ NMR spectroscopy allowed us to scale-up and hasten the preparation: we reduced the reaction time (from ca. 24 to $3 \mathrm{~h}$ ) by increasing the reaction temperature $\left(\right.$ ca. $\left.110{ }^{\circ} \mathrm{C}\right)$ and using toluene as solvent. Ensuring that no $\mathbf{0 P b}$ remained by reaction monitoring enabled simple $\mathbf{1} \mathbf{P b}$ sublimation. We obtained reduced yield ( $51 \%$ on a $10 \mathrm{~g}$ scale), but larger quantities of pure $\mathbf{1 P b}$ could be prepared in a timely manner. Optimization of the kinetic conditions for this reaction currently lies outside the scope of this work but is the subject of ongoing research.

\subsubsection{PbS Film Growth}

(a)
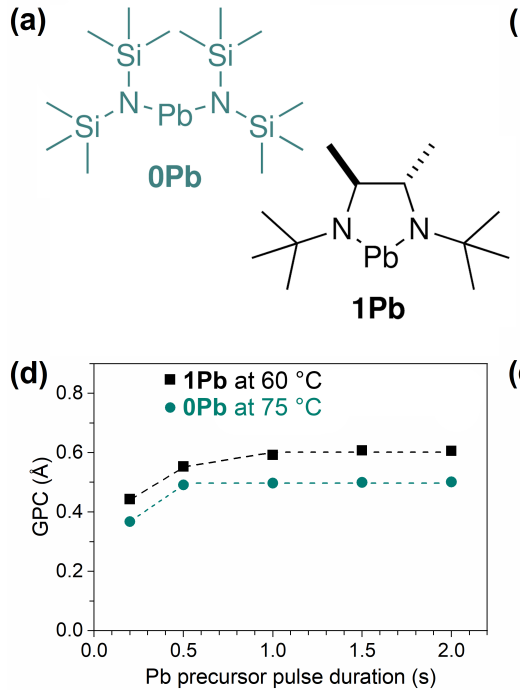

(b)
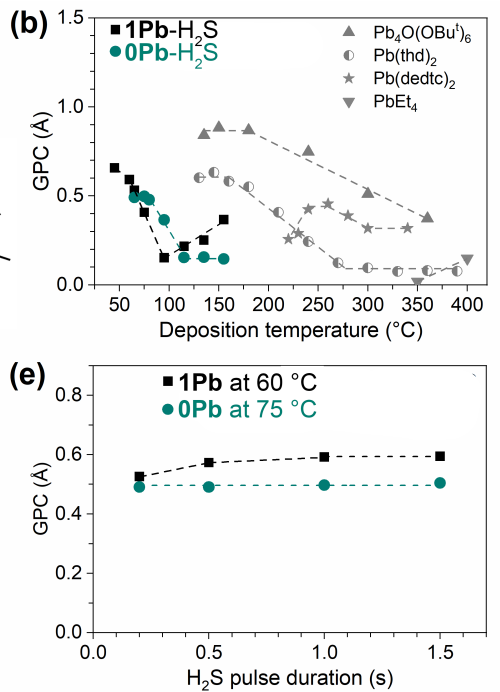
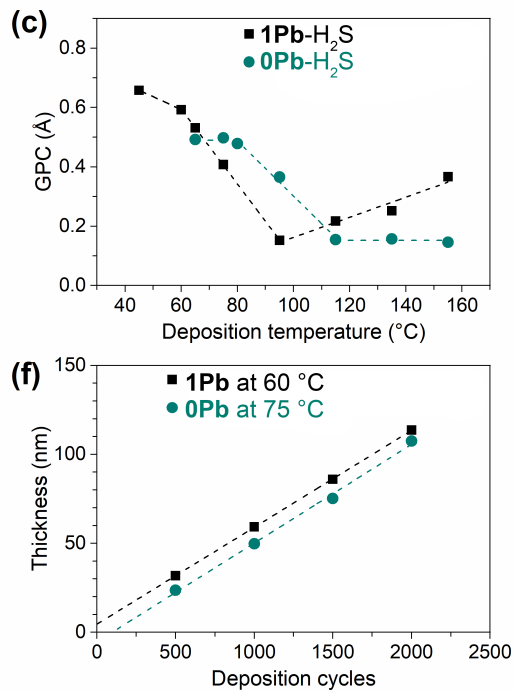

Figure 3.1: (a) Lead precursors used in this study. (b) Overview of ALD processes for PbS with $\mathrm{H}_{2}$ S. For more details on $\mathrm{Pb}_{4} \mathrm{O}\left(\mathrm{O}^{t} \mathrm{Bu}\right)_{6}, \mathrm{~Pb}(\text { tmhd })_{2}$, and $\mathrm{Pb}(\text { dedtc })_{2}$ refer to Nykänen et al. ${ }^{160}$ and on $\mathrm{PbEt}_{4}$ to Yun et al. ${ }^{181}$ Growth per cycle (GPC) of the PbS film on silicon as (c) a function of deposition temperature, (d) Pb precursor pulse duration, and (e) $\mathrm{H}_{2} \mathrm{~S}$ pulse duration. (f) $\mathrm{PbS}$ film thickness on silicon as a function of applied deposition cycles. Unless otherwise evident, data are from films deposited with 1000 cycles, $1 \mathrm{~s}$ precursor pulses, and purge durations. 
Both $\mathbf{0 P b}$ and $\mathbf{1 P b}$, in combination with $\mathrm{H}_{2} \mathrm{~S}$, produced $\mathrm{PbS}$ films at low deposition temperatures (Figure 3.1c). The deposition temperature ranges that we explored were limited by the precursor source temperatures on the lower end and by the precursor decomposition temperatures on the higher end. The $\mathbf{0} \mathbf{P b}-\mathrm{H}_{2} \mathrm{~S}$ process has two regions where the GPC is independent of the deposition temperature. In this process the GPC decreases toward higher temperatures. We attribute the GPC decrease to the surface passivation by $-\mathrm{SiMe}_{3}$ groups, forming from the protonated $-\mathrm{N}\left(\mathrm{SiMe}_{3}\right)_{2}$ ligand, that becomes more effective at higher temperatures. ${ }^{183}$ In the $\mathbf{1} \mathbf{P b}-\mathrm{H}_{2} \mathrm{~S}$ process, at first, the GPC also decreases with increasing deposition temperature, but at temperatures higher than $95{ }^{\circ} \mathrm{C}$ the GPC starts to increase again. This increase is related to the changes in film composition that will be discussed later.
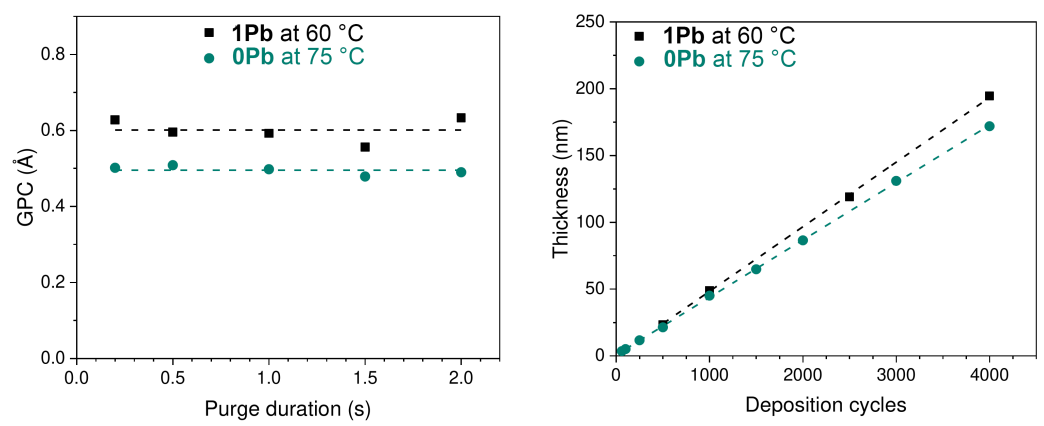

Figure 3.2: (left) GPC as function of the purge duration between precursor pulses. In these experiments precursor pulse durations were fixed $1 \mathrm{~s}$ and the number of cycles at 1000 . Purge duration after both precursor pulses was varied. For example $0.5 \mathrm{~s}$ purge duration corresponds to a process cycle consisting of $1 \mathrm{~s}$ lead precursor pulse, $0.5 \mathrm{~s}$ purge, $1 \mathrm{~s} \mathrm{H}_{2} \mathrm{~S}$ precursor pulse and $0.5 \mathrm{~s}$ purge. (right) $\mathrm{PbS}$ film thickness on sapphire as a function of applied deposition cycles. Films deposited with $1 \mathrm{~s}$ precursor pulses and purge durations.

We studied the influence of the process parameters on the film deposition in more detail at the deposition temperatures of 75 and $60{ }^{\circ} \mathrm{C}$ with $\mathbf{0 P b}$ and $\mathbf{1 P b}$, respectively. At these temperatures, in both processes, the GPC saturates with respect to the $\mathrm{Pb}$ precursor as well as $\mathrm{H}_{2} \mathrm{~S}$ pulse durations (Figure 3.1d,e) and is unaffected by the duration of the nitrogen purge (Figure 3.2). The thickness of the film is straightforwardly adjustable due to its linear dependence on the number of deposition cycles on both silicon (Figure 3.1f) 

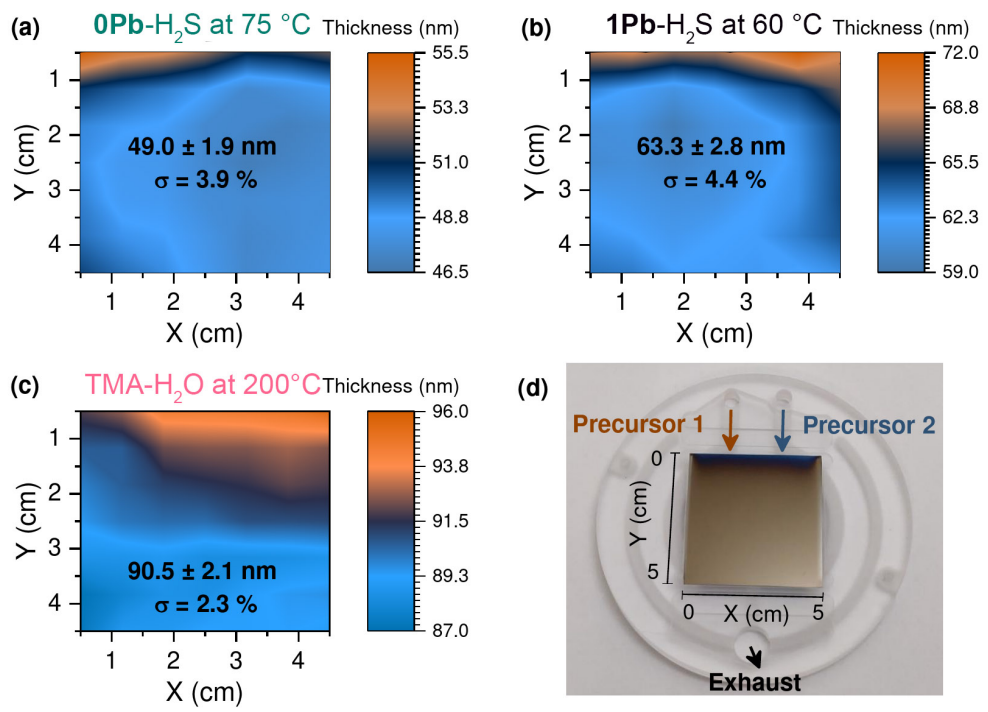

(d)

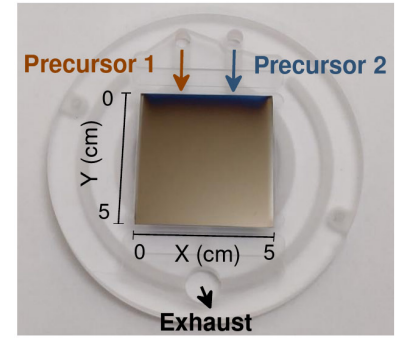

Figure 3.3: XRR thickness maps with $0.5 \mathrm{~cm}$ edge exclusion of films deposited on $5 \mathrm{x} 5 \mathrm{~cm}^{2} \mathrm{Si}$ substrates. a) and b) are measured from $\mathrm{PbS}$ films deposited with 1000 cycles, $1 \mathrm{~s}$ precursor pulses and 2.0 s purge durations with $\mathbf{0 P b}$ at $75{ }^{\circ} \mathrm{C}$ and $1 \mathbf{P b}$ at $60{ }^{\circ} \mathrm{C}$ respectively. c) is from a reference $\mathrm{Al}_{2} \mathrm{O}_{3}$ film deposited with trimethylaluminum (TMA) and water at $200{ }^{\circ} \mathrm{C}$ with 1000 cycles, $1 \mathrm{~s}$ precursor pulse and purge durations. In d) half of the substrate holder of the F120 ALD reactor with precursor inlets and exhaust marked with arrows is shown. If the leading edge (top $1.5 \mathrm{~cm}$ of the film) is excluded from the analysis, the thickness nonuniformities become $1.8 \%, 2.0 \%$ and $1.1 \%$ for $\mathbf{0 P b}-\mathrm{H}_{2} \mathrm{~S}, \mathbf{1} \mathbf{P b}-\mathrm{H}_{2} \mathrm{~S}$, and TMA $-\mathrm{H}_{2} \mathrm{O}$ respectively.

and sapphire (Figure 3.2). The films are surprisingly uniform for such low temperatures and short pulse and purge durations. The thickness nonuniformities over the $5 \times 5 \mathrm{~cm}^{2}$ substrate are $3.9 \%$ and $4.4 \%$ with $\mathbf{0 P b}$ and $\mathbf{1 P b}$, respectively (Figure 3.3). These values are similar to those of the TMA- $\mathrm{H}_{2} \mathrm{O}$ alumina process (2.4\%, Figure 3.3c) and other metal sulfide processes such as $\mathrm{SnS}_{2},{ }^{184} \mathrm{ZrS}_{2}$, and $\mathrm{HfS}_{2}{ }^{185}$ in the same cross-flow F120 ALD reactor but at much higher temperatures.

\subsubsection{Film Composition and Crystallinity}

The $\mathbf{0} \mathbf{P b}-\mathrm{H}_{2} \mathrm{~S}$ process produces crystalline and nearly stoichiometric lead sulfide films in a wide temperature range (Figure 3.4a,b). The films are the purest at the lowest deposition temperatures with a total impurity content of ca. 2 at. \% that increases slightly toward higher deposition temperatures. Also, the $\mathbf{1} \mathbf{P b}-\mathrm{H}_{2} \mathrm{~S}$ process deposits the purest films at the lowest deposition temperatures, but the stoichiometry changes significantly above 
$95{ }^{\circ} \mathrm{C}$ where the films contain more lead than sulfur (Figure 3.4c). XRD patterns of these lead-rich films expose reflections of impurity phases: $\mathrm{PbO}$ and $\mathrm{Pb}_{3}\left(\mathrm{CO}_{3}\right)_{2}(\mathrm{OH})_{2}$ (Figure $3.4 d)$.

(a)

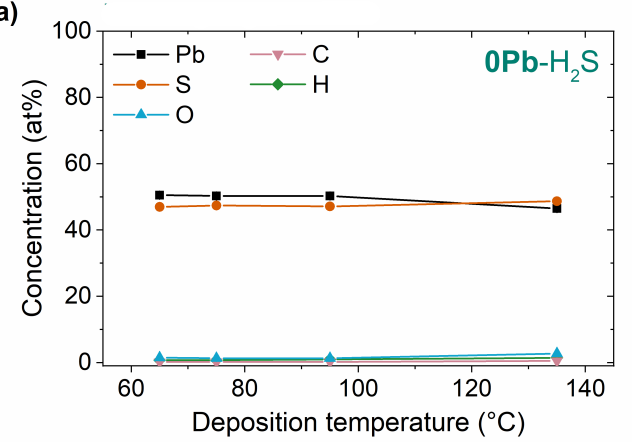

(c)

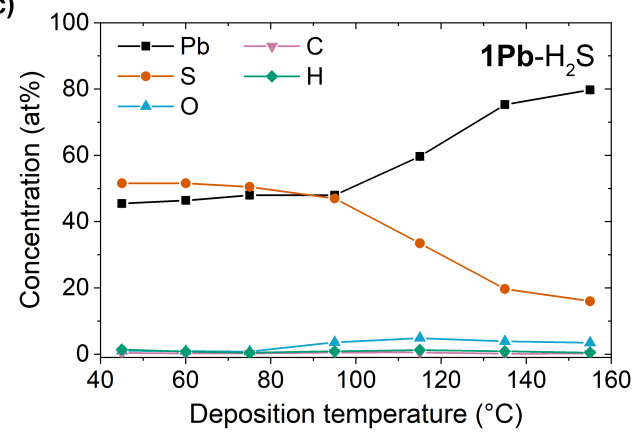

(b)

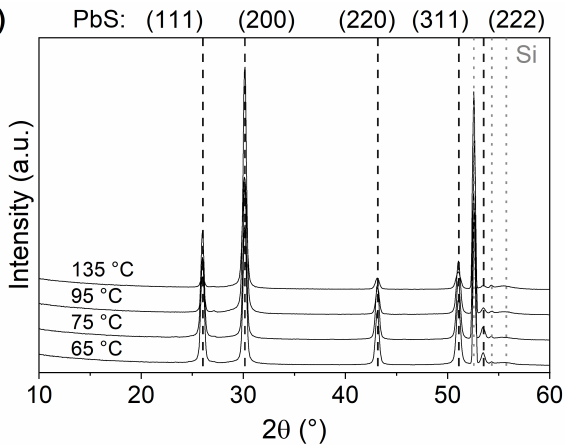

(d)

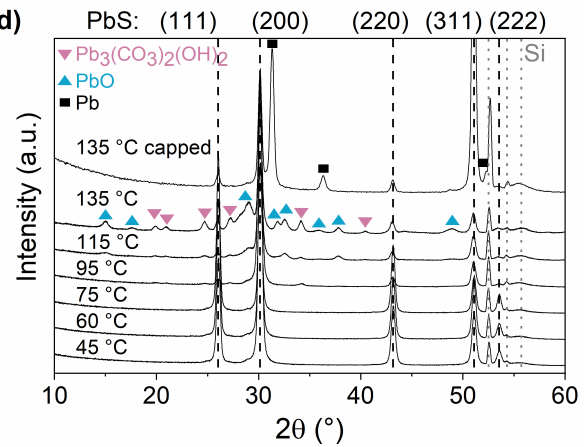

Figure 3.4: TOF-ERDA composition of ca. $100 \mathrm{~nm}$ thick $\mathrm{PbS}$ films deposited at different temperatures with (a) $\mathbf{0 P b}$ and (c) $\mathbf{1 P b}$. For more details on impurity contents see Figure 3.4. Grazing incidence XRD (GIXRD) patterns of PbS films deposited at different temperatures with (b) $\mathbf{0 P b}$ and (d) $\mathbf{1 P b}$. Dashed black and dotted gray lines mark PbS and substrate ( $\mathrm{Si}$ ) reflection positions, respectively. $135{ }^{\circ} \mathrm{C}$ capped refers to a $\mathrm{PbS}$ film capped with ca. $3 \mathrm{~nm} \mathrm{ALD} \mathrm{Al}_{2} \mathrm{O}_{3}$ and measured immediately after the deposition. PbS films were deposited with $1 \mathrm{~s}$ precursor pulses and purge durations. In (b) and (d) films were deposited with 1000 cycles, except for the $135{ }^{\circ} \mathrm{C}$ capped film that was deposited with 2000 cycles.

\subsubsection{Formation of $\mathrm{Pb}$ Using $1 \mathrm{~Pb}$}

The presence of oxygen-containing phases in the XRD patterns was startling because the precursors did not contain oxygen. We speculated that at higher temperatures $\mathbf{1 P b}$ decomposes into elemental lead which would explain the changes in the stoichiometry of the films. When these films are exposed to ambient air, the elemental lead can react with $\mathrm{O}_{2}, \mathrm{CO}_{2}$, or $\mathrm{H}_{2} \mathrm{O}$ in air, producing the oxygen-containing phases observed in the 
XRD patterns. To test this hypothesis, we capped a film deposited at $135{ }^{\circ} \mathrm{C}$ with ca. $3 \mathrm{~nm}$ ALD $\mathrm{Al}_{2} \mathrm{O}_{3}$ (TMA- $\mathrm{H}_{2} \mathrm{O}$ process) without breaking the vacuum and measured the XRD pattern immediately after removing the film from the reactor. Indeed, the XRD pattern of the capped film lacked reflections of the oxygen-containing phases and instead had reflections of elemental lead (Figure 3.4d).

Even though the presence of elemental lead supports our hypothesis on decomposition of $\mathbf{1 P b}$, it is still inconsistent with the higher thermal decomposition temperature $\left(\right.$ ca. $160{ }^{\circ} \mathrm{C}$ ) reported for $\mathbf{1 P b}$ in the literature ${ }^{182}$-our own observation that signs of $\mathbf{1 P b}$ thermal decomposition (blackening of the hot end of the precursor source tube) in the F120 ALD reactor appear only at $\geq 155^{\circ} \mathrm{C}$-and an experiment where at $135{ }^{\circ} \mathrm{C}$ we pulsed only $\mathbf{1 P b}$ without $\mathrm{H}_{2} \mathrm{~S}$ that yielded no deposit at all. Additionally, a set of depositions at $135{ }^{\circ} \mathrm{C}$ revealed that the process still works in an ALD regime with GPC saturating with re-
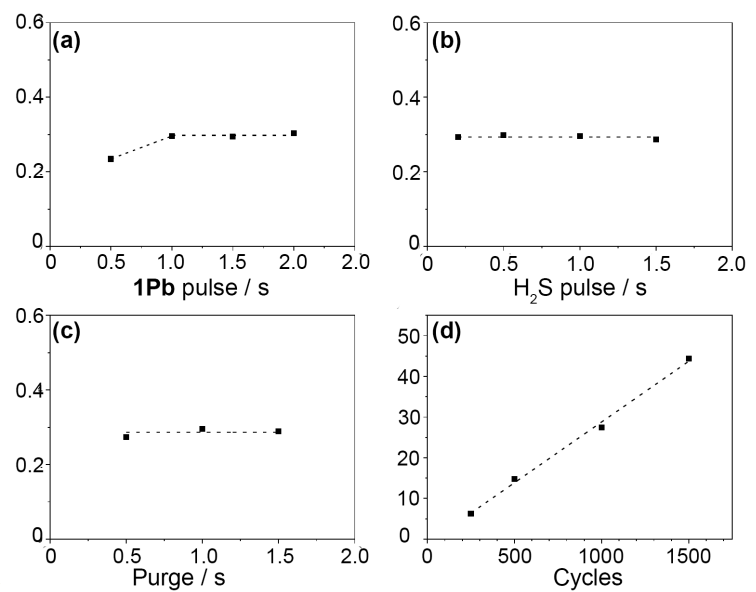

Figure 3.5: $\mathrm{GPC}$ of the $\mathrm{Pb}$ film deposited at $135^{\circ} \mathrm{C}$ as a function of (a) $\mathbf{1 P b}$ pulse, (b) $\mathrm{H}_{2} \mathrm{~S}$ pulse and (c) purge durations. (d) shows $\mathrm{PbS}_{x}$ film thickness on silicon as a function of cycles. Other films were deposited with 1500 cycles, 1 s precursor pulses and purge durations. In (c) purge duration after both precursor pulses were varied. A thin $\mathrm{Al}_{2} \mathrm{O}_{3}$ underlayer (ca. $1.6 \mathrm{~nm}, 25$ cycles) and overlayer (ca. $3 \mathrm{~nm}, 50$ cycles) was deposited by ALD with TMA- $\mathrm{H}_{2} \mathrm{O}$ (1 s pulse, $4 \mathrm{~s}$ purge). spect to precursor pulse durations and films being visually uniform (Figures 3.5 and 3.6e). These observations suggest that elemental lead is formed by a mechanism that involves $\mathrm{H}_{2} \mathrm{~S}$ rather than thermal decomposition of $\mathbf{1 P b}$. In $\mathbf{1 P b}$ lead has an oxidation state of +2 , and therefore lead ion must be reduced and another species must be oxidized. We speculated that the the oxidized species is sulfide $\left(\mathrm{S}^{2-}\right)$ and that it oxidizes to elemental sulfur, which is volatile enough to be removed during the deposition. It is not uncommon for $\mathrm{H}_{2} \mathrm{~S}$ to act as a reducing agent in ALD of transition metal dichalcogenides and reduce 
the metal ions in metal pentachlorides such as $\mathrm{MoCl}_{5}{ }^{186}$ and $\mathrm{ReCl}_{5}{ }^{187}$ to a lower oxidation state. This speculation is further supported by the fact that sulfur is sufficiently volatile at the temperatures and conditions being discussed; for example, Tripathi et al. used elemental sulfur as a precursor at a source temperature of $115{ }^{\circ} \mathrm{C}$ in ALD of copper sulfide, ${ }^{188}$ in the same F120 ALD reactor as used here.

While reduction by $\mathrm{H}_{2} \mathrm{~S}$ accounts for some of our empirical observations, it does not explain the difference between the two lead precursors. To understand the cause of that difference, we evaluated the thermodynamics of the reduction with gas-phase density functional theory (DFT, def2-QZVPP// $\omega$ B97X-D3(BJ)/def2-SVP), correcting for the gasto-solid transition of $\mathrm{Pb}$ and $\mathrm{PbS}$ with literature thermochemical data. ${ }^{189}$ These calculations indicate that reduction with $\mathrm{H}_{2} \mathrm{~S}$ with concomitant elimination of elemental sulfur is nonspontaneous, in direct contradiction to the initially proposed mechanism (Table 3.1). To elucidate a plausible mechanism for the elemental lead formation, we then considered several other reactions such as film etching by the free ligand and formation of sulfur diamides. Of these candidates, only one fit with our empirical observations by requiring $\mathrm{H}_{2} \mathrm{~S}$ and being spontaneous for $\mathbf{1} \mathbf{P b}$ but not $\mathbf{0 P b}$. That reaction is similar to the precursor thermolysis that yields elemental lead and $\mathrm{MeC}(\mathrm{H})=\mathrm{N}^{t} \mathrm{Bu}$ (nonspontaneous, $\Delta G=$ $56 \mathrm{~kJ} / \mathrm{mol}$ ); however, the consequent thiolysis of the imine to produce thioacetaldehyde which further oligomerizes to a heterocyclic trithiane makes this pathway spontaneous $(\Delta G=-10 \mathrm{~kJ} / \mathrm{mol}$, Figure 3.7). While this speculative mechanism fulfills all the requirements, we emphasize that it, or any alternative, would require a dedicated in situ mechanism study for verification. We believe that such a study, while outside of the scope of this work, would improve understanding of processes occurring in low-temperature deposition of lead materials with amide precursors.

The possibility of depositing elemental $\mathrm{Pb}$ with ALD at low temperatures without using a dedicated reducing agent is intriguing. We briefly explored whether that is possible by adjusting the process parameters. The $\mathrm{PbS}$ to $\mathrm{Pb}$ intensity ratios were unaffected by 

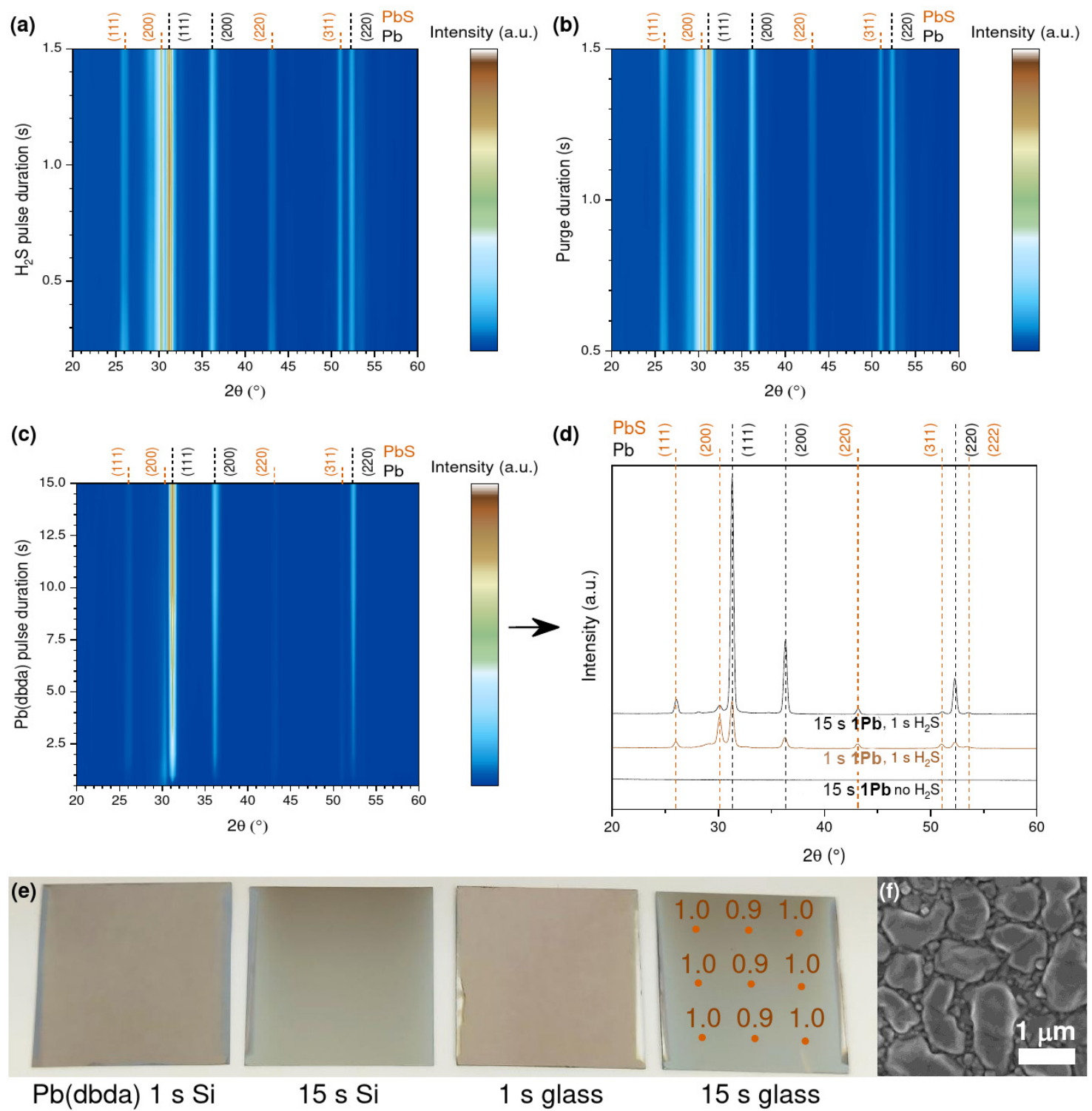

Figure 3.6: Contour profile maps of grazing-incidence XRD (GIXRD) data for $\mathrm{PbS}_{x}$ films deposited on silicon at $135^{\circ} \mathrm{C}$ as a function of (a) $\mathrm{H}_{2} \mathrm{~S}$ pulse duration, (b) purge durations and (c) $\mathbf{1 P b}$ precursor pulse duration. (d) shows GIXRD of films deposited with $15 \mathrm{~s}$ and $1 \mathrm{~s} \mathbf{1 P b}$ pulse durations as well as a reference where only $\mathbf{1 P b}$ was pulsed for $15 \mathrm{~s}$ separated by $1 \mathrm{~s}$ purges. (e) Photographs of $\mathrm{PbS}_{x}$ films deposited on $\mathrm{Si}$ and soda lime glass with $1 \mathrm{~s}$ and $15 \mathrm{~s} \mathbf{1 P b}$ pulse durations. Vermillion dots and numbers are resistivities in $\mathrm{m} \Omega \mathrm{cm}$ measured at that site. (f) FESEM image of a $\mathrm{PbS}_{x}$ film deposited with $15 \mathrm{~s}$ long $\mathbf{1 P b}$ pulse durations. Unless otherwise evident, data is from films deposited with 1500 cycles, $1 \mathrm{~s}$ precursor pulses and purge durations. In (c) purge duration after both precursor pulses were varied. In all depositions a thin $\mathrm{Al}_{2} \mathrm{O}_{3}$ underlayer (ca. $1.6 \mathrm{~nm}, 25$ cycles) and overlayer (ca. $3 \mathrm{~nm}, 50$ cycles) was deposited by ALD with TMA- $\mathrm{H}_{2} \mathrm{O}$ ( $1 \mathrm{~s}$ pulse, $4 \mathrm{~s}$ purge) before and after the $\mathrm{PbS}$ deposition. 


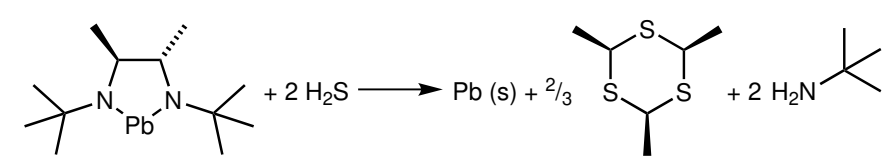

Figure 3.7: Spontaneous reduction of $\mathbf{1 P b}$ to $\mathrm{Pb}(0)$ by $\mathrm{H}_{2} \mathrm{~S}$ and formation of the cyclic, in this case more stable, cis-1,3,5-trithiane.

Table 3.1: Thermodynamics of possible reactions during ALD of $\mathrm{PbS}$ with $\mathbf{0 P b}$ or $\mathbf{1} \mathbf{P b} .^{\text {a }}$

\begin{tabular}{|c|c|c|c|}
\hline Reaction $^{b}$ & $\begin{array}{c}\Delta H^{\ominus} \\
\mathrm{kcal} / \mathrm{mol}\end{array}$ & $\begin{array}{c}\Delta S^{\ominus} \\
\mathrm{kcal} / \mathrm{Kmol}\end{array}$ & $\begin{array}{c}\Delta G^{\ominus} \\
\mathrm{kcal} / \mathrm{mol}\end{array}$ \\
\hline \multicolumn{4}{|l|}{$A L D$} \\
\hline $\mathbf{0 P b}+\mathrm{H}_{2} \mathrm{~S} \longrightarrow \mathrm{PbS}+2\left(\mathrm{Me}_{3} \mathrm{Si}\right)_{2} \mathrm{NH}$ & -55.39 & 0.0177 & -60.65 \\
\hline $\mathbf{1 P b}+\mathrm{H}_{2} \mathrm{~S} \longrightarrow \mathrm{PbS}+\operatorname{rac}-\left[\mathrm{MeC}(\mathrm{H}) \mathrm{NH}^{t} \mathrm{Bu}\right]_{2}^{\mathrm{c}}$ & -71.73 & -0.0241 & -64.55 \\
\hline \multicolumn{4}{|l|}{ Reduction by $\mathrm{H}_{2} \mathrm{~S}$} \\
\hline $\mathbf{0 P b}+\mathrm{H}_{2} \mathrm{~S} \longrightarrow \mathrm{Pb}+2\left(\mathrm{Me}_{3} \mathrm{Si}\right)_{2} \mathrm{NH}+\frac{1}{8} \mathrm{~S}_{8}$ & 2.13 & -0.0174 & 7.33 \\
\hline $\mathbf{1 P b}+\mathrm{H}_{2} \mathrm{~S} \longrightarrow \mathrm{Pb}+r a c-\left[\mathrm{MeC}(\mathrm{H}) \mathrm{NH}^{t} \mathrm{Bu}\right]_{2}+\frac{1}{8} \mathrm{~S}_{8}$ & -9.69 & -0.0710 & 11.48 \\
\hline \multicolumn{4}{|l|}{ Reduction by $\mathrm{H}_{2}$} \\
\hline $\mathbf{0 P b}+\mathrm{H}_{2} \longrightarrow \mathrm{Pb}+2\left(\mathrm{Me}_{3} \mathrm{Si}\right)_{2} \mathrm{NH}$ & -5.22 & -0.0110 & -1.93 \\
\hline $\mathbf{1 P b}+\mathrm{H}_{2} \longrightarrow \mathrm{Pb}+$ rac $-\left[\mathrm{MeC}(\mathrm{H}) \mathrm{NH}^{t} \mathrm{Bu}\right]_{2}$ & -17.04 & -0.0646 & 2.22 \\
\hline \multicolumn{4}{|l|}{ Etching by plumbylene } \\
\hline $\mathbf{0 P b}+\mathrm{PbS} \longrightarrow 2 \mathrm{~Pb}+\mathbf{0 S}^{\mathrm{d}}$ & 92.82 & -0.0756 & 115.35 \\
\hline $\mathbf{1 P b}+\mathrm{PbS} \longrightarrow 2 \mathrm{~Pb}+\mathbf{1 S}^{\mathrm{d}}$ & 88.38 & -0.0775 & 111.49 \\
\hline \multicolumn{4}{|l|}{ Etching by free amine } \\
\hline $\mathrm{PbS}+2\left(\mathrm{Me}_{3} \mathrm{Si}\right)_{2} \mathrm{NH} \longrightarrow \mathrm{Pb}+\mathrm{H}_{2}+\mathbf{O S}$ & 98.04 & -0.0645 & 117.28 \\
\hline $\mathrm{PbS}+\operatorname{rac}-\left[\mathrm{MeC}(\mathrm{H}) \mathrm{NH}^{t} \mathrm{Bu}\right]_{2} \longrightarrow \mathrm{Pb}+\mathrm{H}_{2}+\mathbf{1 S}$ & 105.42 & -0.0129 & 109.27 \\
\hline $\mathrm{PbS}+\operatorname{rac}-\left[\mathrm{MeC}(\mathrm{H}) \mathrm{NH}^{t} \mathrm{Bu}\right]_{2} \longrightarrow \mathrm{Pb}+\mathrm{H}_{2} \mathrm{~S}+2 \mathrm{MeC}(\mathrm{H})=\mathrm{N}^{t} \mathrm{Bu}$ & 86.75 & 0.0570 & 69.75 \\
\hline \multicolumn{4}{|l|}{$\mathbf{1 P b}$ thermolysis } \\
\hline $\mathbf{1 P b} \longrightarrow \mathrm{Pb}+2 \mathrm{MeC}(\mathrm{H})=\mathrm{N}^{t} \mathrm{Bu}^{\mathrm{e}}$ & 19.60 & 0.0211 & 13.31 \\
\hline$r a c-\left[\mathrm{MeC}(\mathrm{H}) \mathrm{NH}^{t} \mathrm{Bu}\right]_{2} \longrightarrow 2 \mathrm{MeC}(\mathrm{H})=\mathrm{N}^{t} \mathrm{Bu}+\mathrm{H}_{2}$ & 36.64 & 0.0857 & 11.09 \\
\hline $\mathbf{1 P b}+\operatorname{rac}-\left[\mathrm{MeC}(\mathrm{H}) \mathrm{NH}^{t} \mathrm{Bu}\right]_{2} \longrightarrow \mathrm{Pb}+\mathrm{H}_{2}+4 \mathrm{MeC}(\mathrm{H})=\mathrm{N}^{t} \mathrm{Bu}$ & 56.25 & 0.1068 & 24.40 \\
\hline \multicolumn{4}{|l|}{ Thiolysis } \\
\hline $\mathrm{H}_{2} \mathrm{~S}+\mathrm{MeC}(\mathrm{H})=\mathrm{N}^{t} \mathrm{Bu} \longrightarrow \mathrm{MeCH}=\mathrm{S}^{f_{+}}{ }^{t} \mathrm{BuNH}_{2}$ & 6.53 & 0.0027 & 5.74 \\
\hline $\mathrm{H}_{2} \mathrm{~S}+\mathrm{MeC}(\mathrm{H})=\mathrm{N}^{t} \mathrm{Bu} \longrightarrow \frac{1}{3}$ cis- $[\mathrm{MeC}(\mathrm{H}) \mathrm{S}]_{3} \mathrm{~g}_{+}{ }^{t} \mathrm{BuNH}_{2}$ & -16.35 & -0.0289 & -7.72 \\
\hline $\mathrm{H}_{2} \mathrm{~S}+\mathrm{MeC}(\mathrm{H})=\mathrm{N}^{t} \mathrm{Bu} \longrightarrow \frac{1}{3}$ trans $-[\mathrm{MeC}(\mathrm{H}) \mathrm{S}]_{3}+{ }^{t} \mathrm{BuNH}_{2}$ & -15.74 & -0.0293 & -7.01 \\
\hline $\mathbf{1 P b}+2 \mathrm{H}_{2} \mathrm{~S} \longrightarrow \mathrm{Pb}+\frac{2}{3}$ cis- $[\mathrm{MeC}(\mathrm{H}) \mathrm{S}]_{3}+2{ }^{t} \mathrm{BuNH}_{2}$ & -13.10 & -0.0368 & -2.13 \\
\hline $\mathbf{1 P b}+2 \mathrm{H}_{2} \mathrm{~S} \longrightarrow \mathrm{Pb}+\frac{2}{3}$ trans $-[\mathrm{MeC}(\mathrm{H}) \mathrm{S}]_{3}+2{ }^{t} \mathrm{BuNH}_{2}$ & -11.87 & -0.0374 & -0.71 \\
\hline \multicolumn{4}{|l|}{ Miscellaneous } \\
\hline $\mathrm{H}_{2} \mathrm{~S} \longrightarrow \mathrm{H}_{2}+\frac{1}{8} \mathrm{~S}_{8}$ & 7.35 & -0.00640 & 9.25 \\
\hline $\mathrm{PbS} \longrightarrow \mathrm{Pb}+\frac{1}{8} \mathrm{~S}_{8}$ & 57.52 & -0.03510 & 67.98 \\
\hline $\mathrm{PbS}(\mathrm{g}) \longrightarrow \mathrm{PbS}(\mathrm{s})$ & -55.00 & -0.03826 & -43.59 \\
\hline $\mathrm{Pb}(\mathrm{g}) \longrightarrow \mathrm{Pb}(\mathrm{s})$ & -46.70 & -0.02643 & -38.82 \\
\hline
\end{tabular}

${ }^{a}$ Calculated with density functional theory def2-QZVPP// $\omega$ B97X-D3(BJ)/def2-SVP.

${ }^{\mathrm{b}} \mathrm{Pb}$ and $\mathrm{PbS}$ are solids in all reactions they are involved in, with the solid-to-gas transition added to the DFT energies according to Hess's law. Standard enthalpies, entropies, and energies of the phase changes are from the literature. All other species are in the gas phase.

${ }^{c}$ rac- $N, N^{\prime}$-di-tert-butyl-2,3-diaminobutane

${ }^{\mathrm{d}}$ Analogous to $\mathrm{Pb}, \mathbf{0 S}$ is sulfur(II) bis[bis(trimethylsilylamide)] and $\mathbf{1 S}$ is sulfur(II) $r a c-N, N^{\prime}$-di-tert-butylbutane-2,3-diamide

e $N$-tert-butylacetaldimine

${ }^{\mathrm{f}}$ Thioacetaldehyde

g 2,4,6-trimethyl-1,3,5-trithiane 
changes in the $\mathrm{H}_{2} \mathrm{~S}$ pulse or purge durations (Figure 3.6a,b). In contrast, increasing the $\mathbf{1 P b}$ pulse duration increased the intensity from the $\mathrm{Pb}$ phase (Figure 3.6c,d). However, even with $15 \mathrm{~s}$ pulse duration some $\mathrm{PbS}$ was still present. The film with the highest $\mathrm{Pb}$ content had a low resistivity of $1.0 \mathrm{~m} \Omega \mathrm{cm}(\mathrm{Pb}$ bulk resistivity $0.02 \mathrm{~m} \Omega \mathrm{cm})$, resembled rough metallic films in appearance, and consisted of large micrometer-sized grains connected by smaller submicrometer-sized grains (Figure 3.6e,f).

\subsubsection{Film Morphology}
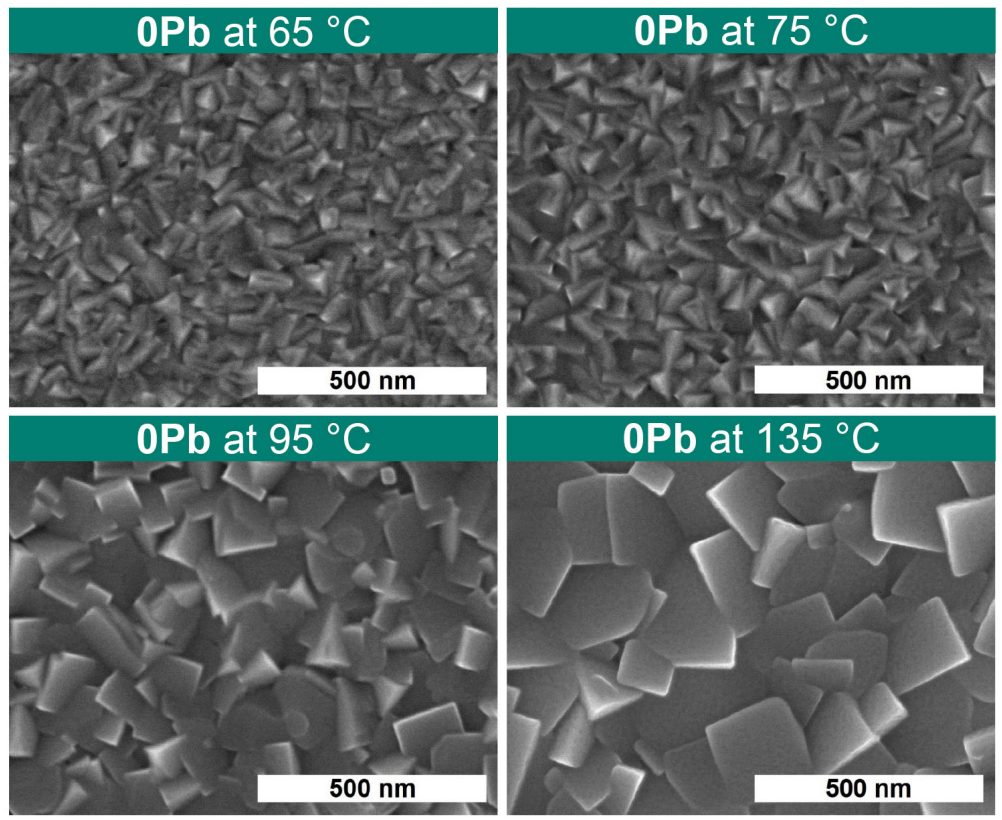

Figure 3.8: FESEM images of $\mathrm{PbS}$ films deposited at different temperatures with the $\mathbf{0} \mathbf{P b}-\mathrm{H}_{2} \mathrm{~S}$ process using $1 \mathrm{~s}$ precursor pulses and purge durations. The number of deposition cycles was chosen according to Figure 3.1c so that the target thickness was approximately $100 \mathrm{~nm}$.

Formation of elemental lead at higher temperatures in the $\mathbf{1} \mathbf{P b}-\mathrm{H}_{2} \mathrm{~S}$ process affects also the morphology of the films. Films deposited at lower temperatures by both processes consist of small cube-shaped grains (Figure 3.11a) as is characteristic to PbS. The grains increase in size with increasing deposition temperature in the $\mathbf{0} \mathbf{P b}-\mathrm{H}_{2} \mathrm{~S}$ process (Figure 3.8), which linearly increases the roughness of the films with the deposition temperature (Figure $3.11 \mathrm{~b}$ ). In the $\mathbf{1} \mathbf{P b ~} \mathrm{H}_{2} \mathrm{~S}$ process, in addition to the cubic grains growing in size, 
irregularly shaped lumps start to appear (Figure 3.9) at higher deposition temperatures. These lumps increase in size more rapidly with the deposition temperature than the cubic grains, and this in turn causes an exponential increase in the roughness of the films (Figure $3.11 b)$.
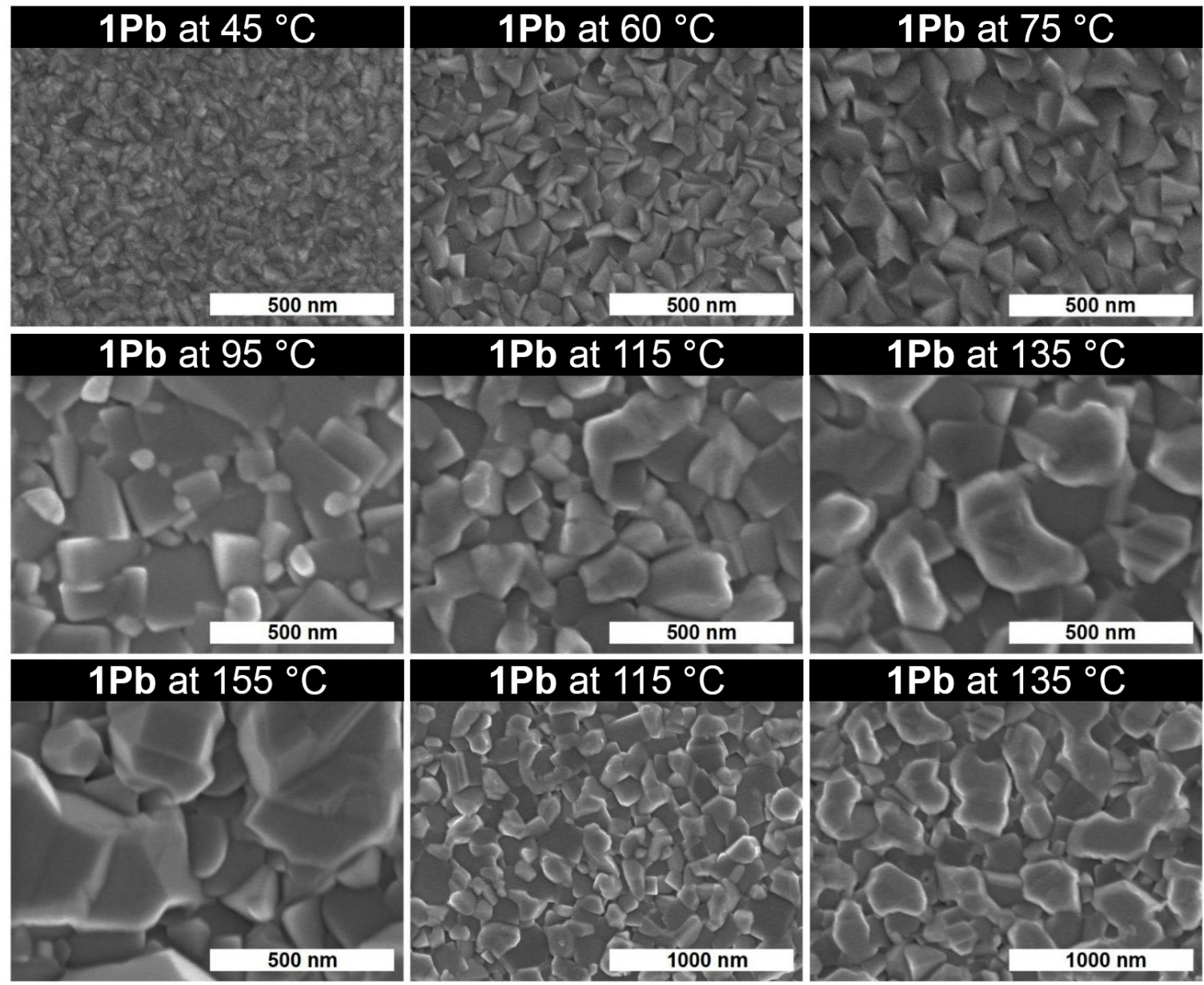

Figure 3.9: FESEM images of $\mathrm{PbS}$ films deposited at different temperatures with the $\mathbf{1} \mathbf{P b}-\mathrm{H}_{2} \mathrm{~S}$ process using $1 \mathrm{~s}$ precursor pulses and purge durations. The number of deposition cycles was chosen according to Figure 1c so that the target thickness was approximately $100 \mathrm{~nm}$. Note different magnifications in (h) and (i). 

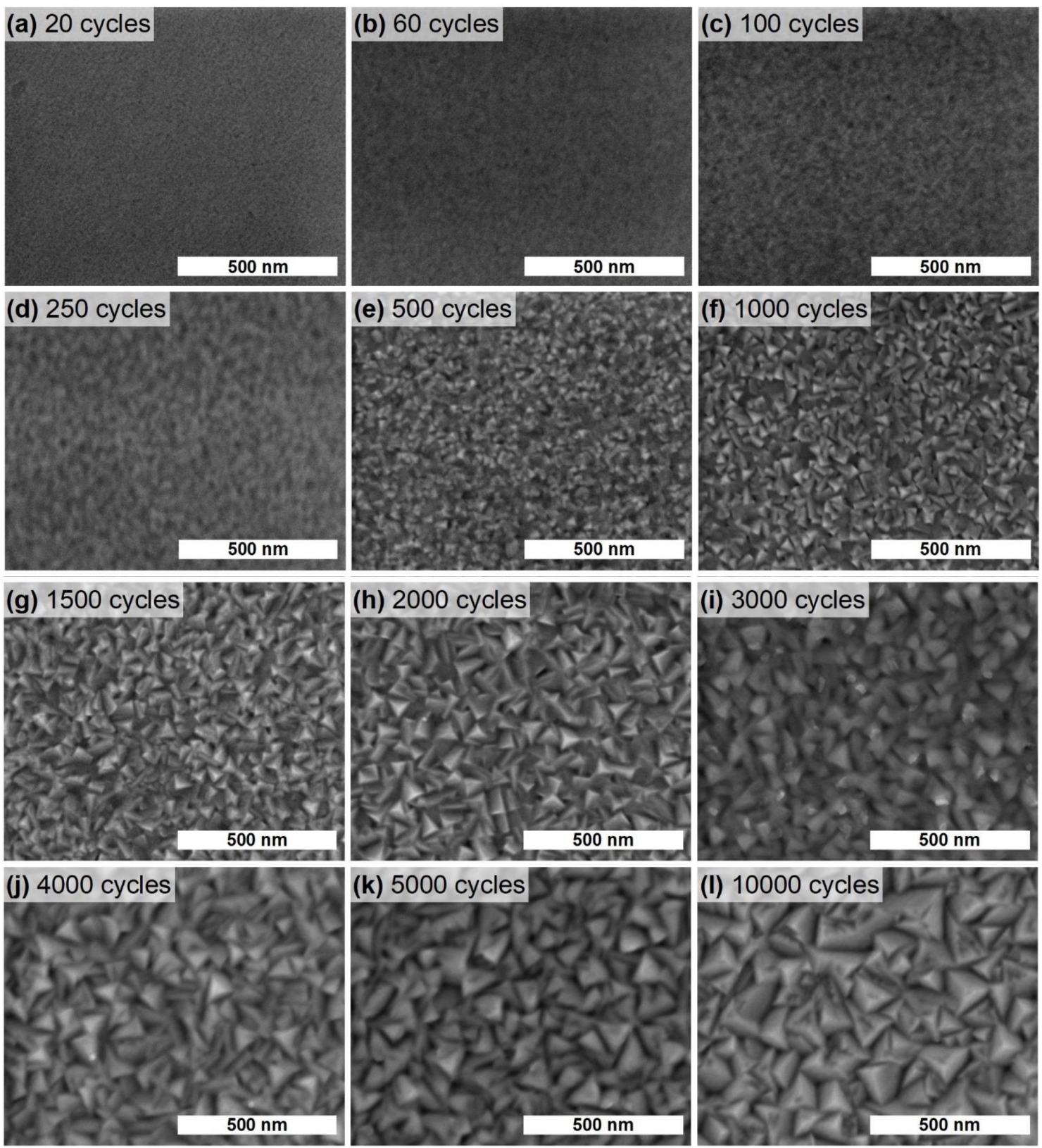

Figure 3.10: FESEM images of $\mathrm{PbS}$ films deposited with different number of cycles using $\mathbf{0} \mathbf{P b}-\mathrm{H}_{2} \mathrm{~S}$. The films were deposited at $75{ }^{\circ} \mathrm{C}$ process using $1 \mathrm{~s}$ precursor pulses and purge durations. 


\subsubsection{Electrical Properties}

Hall effect measurements revealed that both processes yield conductive $p$-type PbS films (Figure 3.11c). The hole mobilities are $10-70 \mathrm{~cm}^{2} \mathrm{~V}^{-1} \mathrm{~s}^{-1}$ (Figure 3.11d) and are comparable to or exceed the mobilities of semiconductors typically used in perovskite based devices, both organic such as PEDOT:PSS $\left(2 \mathrm{~cm}^{2} \mathrm{~V}^{-1} \mathrm{~s}^{-1}\right)^{190}$ and spiro-MeOTAD $\left(2 \times 10^{-3} \mathrm{~cm}^{2} \mathrm{~V}^{-1} \mathrm{~s}^{-1}\right)^{191}$ as well as inorganic such as $\mathrm{NiO}\left(10 \mathrm{~cm}^{2} \mathrm{~V}^{-1} \mathrm{~s}^{-1}\right) .{ }^{192}$

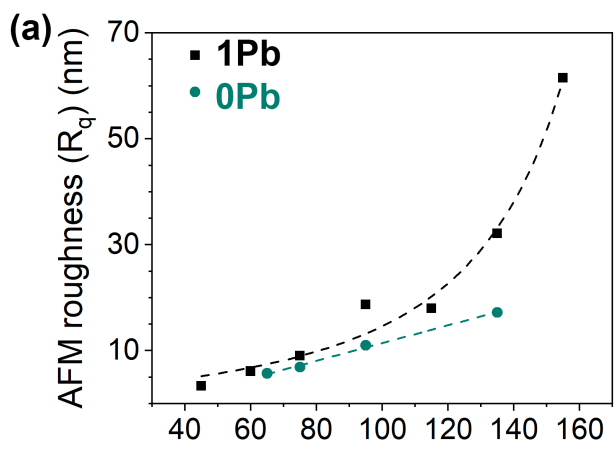

(c)

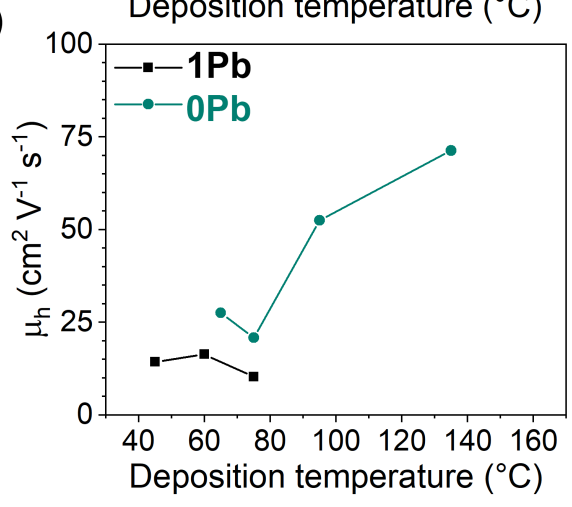

(b)

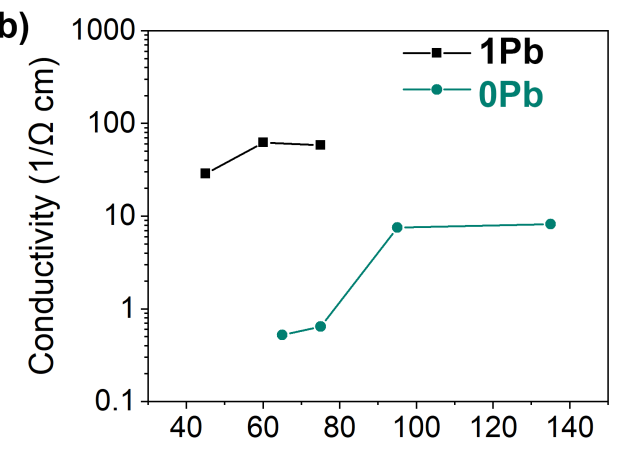

(d)

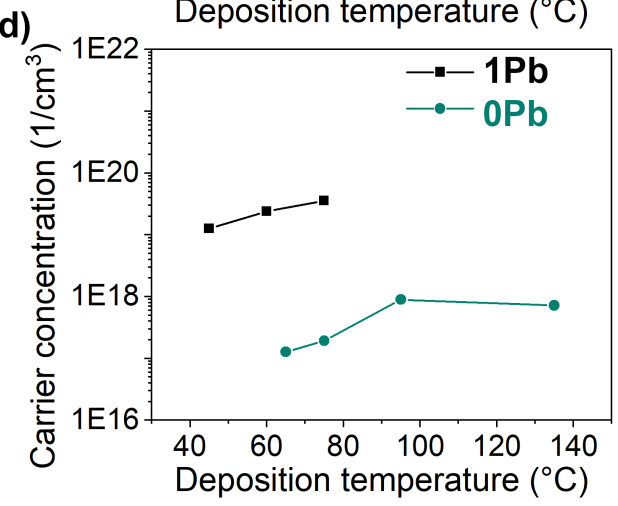

Figure 3.11: (a) AFM roughness $R_{q}$, (b) conductivity, (c) hole mobility, and (d) carrier concentration of the $\mathrm{PbS}$ films as a function of deposition temperature. The substrate was silicon in (b) and sapphire in (e). Films were ca. $100 \mathrm{~nm}$ thick and were deposited with $1 \mathrm{~s}$ precursor pulses and purge durations.

\subsubsection{1 $0 \mathrm{~Pb}$}

In the $\mathrm{PbS}$ films deposited with $\mathbf{0 P b}$, hole mobility, conductivity, and carrier concentration increase at higher deposition temperatures (Figure 3.11c-e). We attribute the changes to the enlargement of grains (Figure 3.11a) and to the increase of sulfur and oxygen concentrations in the films (Figure 3.4a, Figure 3.4a, and Table S2). Enlargement of grains reduces 
grain boundary density, which in turn increases mobility, ${ }^{193,194}$ whereas the excess of sulfur and oxygen result in defects that increase hole concentrations. ${ }^{15,195}$ All chalcogens, when in excess, are known to be $p$-type dopants in lead chalcogenides. ${ }^{196,197}$ The observed changes in composition are on the order of $10^{20}$ atoms $/ \mathrm{cm}^{3}$ and are larger by a factor of 100 compared to the observed carrier concentrations. We speculate that this difference could be caused by carbon and hydrogen impurities because defects created by these impurities can decrease hole concentration. Overall, the observed carrier concentrations are a result of all defects created by nonstoichiometry and impurities. ${ }^{198}$ However, describing the interactions of these defects and their effect on carrier concentration is beyond this study.

\subsubsection{2 $1 \mathrm{~Pb}$}

The presence of several phases in the films deposited with $\mathbf{1 P b}$ at higher temperatures (Figure 3.4d) prevents drawing unambiguous conclusions about their electrical properties. At lower deposition temperatures the changes in grain sizes and composition are not significant enough (Figure 3.4c and Figure 3.9a-c) to cause major differences in electrical properties of these films (Figure 3.11c-e). Compared to $\mathbf{0 P b}, \mathbf{1 P b}$ yields films with a conductivity larger by a factor of 100 (Figure 3.11c). The difference in conductivity arises from a difference in carrier concentration (Figure 3.11e). We attribute these differences to the excess of sulfur and lower impurity contents in the films made with $\mathbf{1 P b}$ at lower temperatures (Figure 3.4c and Figure 3.4b). The differences in the electrical properties between the two ALD processes offers flexibility for the design of devices based on semiconductor junctions. Various postdeposition annealing treatments can provide additional control over the electrical properties of $\mathrm{PbS} .{ }^{199}$ 


\subsubsection{Optical Properties}
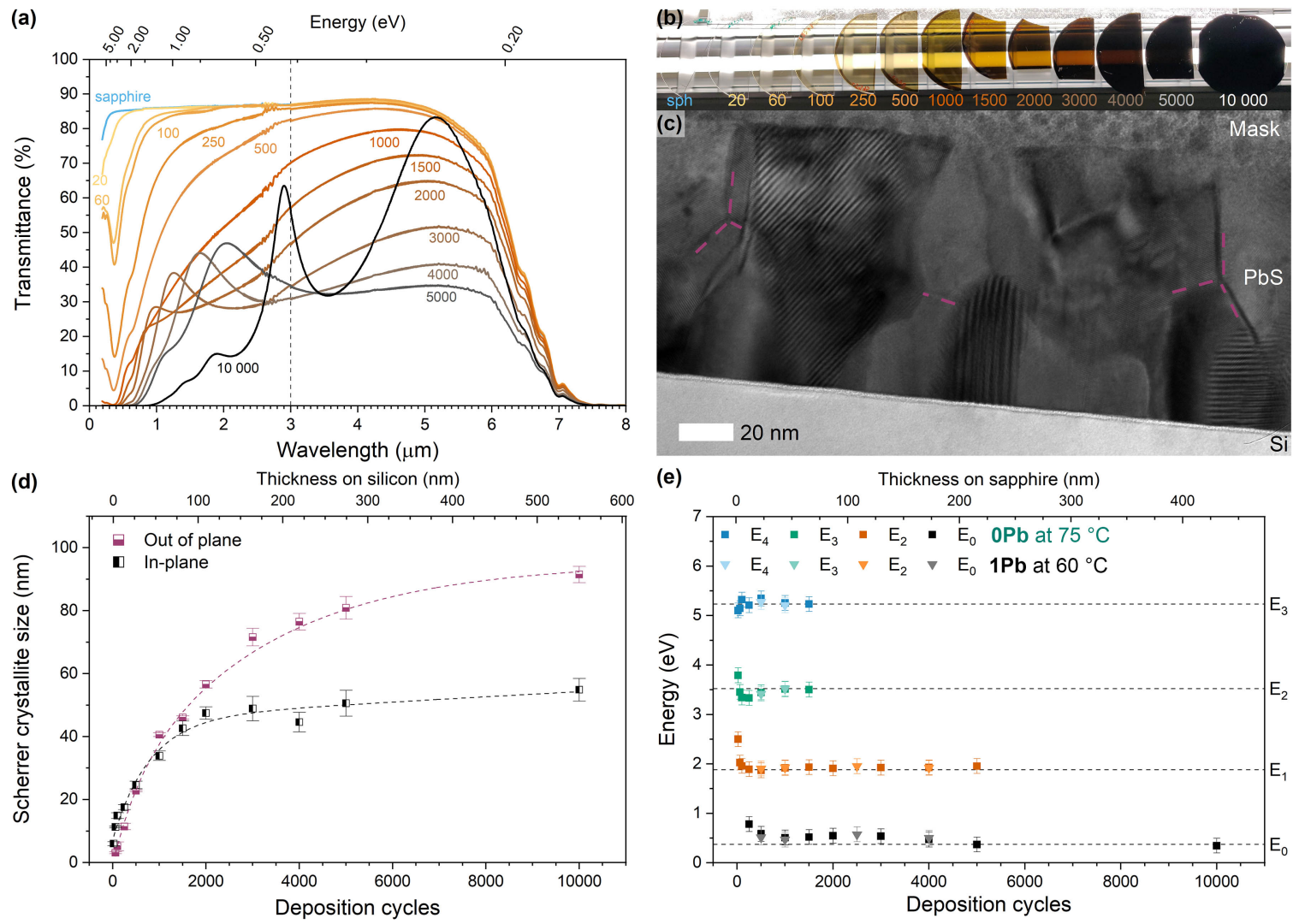

Figure 3.12: (a) Transmittance of $\mathrm{PbS}$ films deposited on sapphire with $\mathbf{0 P b}$ and different number of deposition cycles at $75{ }^{\circ} \mathrm{C}$. The dashed gray line separates wavelength ranges measured with different instruments. (b) Photograph of the films in (a) viewed against an office lamp. (c) Crosssection TEM image of a PbS film deposited with $\mathbf{0 P b}$ at $75{ }^{\circ} \mathrm{C}$ with 2000 cycles. Some grain boundaries are marked with dashed lines. (d) Out-of-plane and in-plane crystallite sizes, as measured with XRD, in PbS films deposited with $\mathbf{0 P b}$ at $75{ }^{\circ} \mathrm{C}$ with different number of cycles. (e) Transition energies defined from $\log (1 / T)$ plots (Figure $3.15 \mathrm{a}, \mathrm{c}$ ), including the band gap $E_{0}$. Dashed gray lines are reference energy values by Cardona and Greenaway. ${ }^{200}$ All films were deposited with 1 s pulse and purge durations.

We expected that the large exciton radius (ca. $20 \mathrm{~nm}$ ) of $\mathrm{PbS}$, its narrow band gap, and the dependence of the grain size on the deposition temperature in our processes would allow us to deposit films with tailorable optical properties. To explore this hypothesis, we measured transmittance of $\mathrm{PbS}$ films deposited on sapphire in the 190-8000 nm range (Figure 3.12a,b and Figure 3.13a-c) and determined their optical band gaps. Optical band gaps are commonly determined from Tauc plots constructed from transmittance data, and 

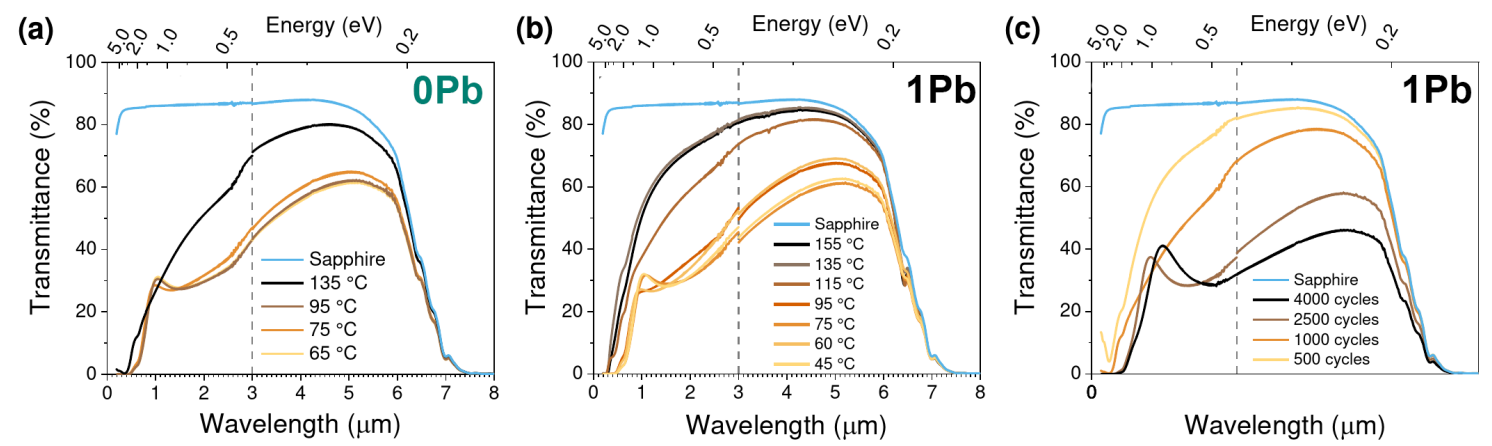

(d)

(e)
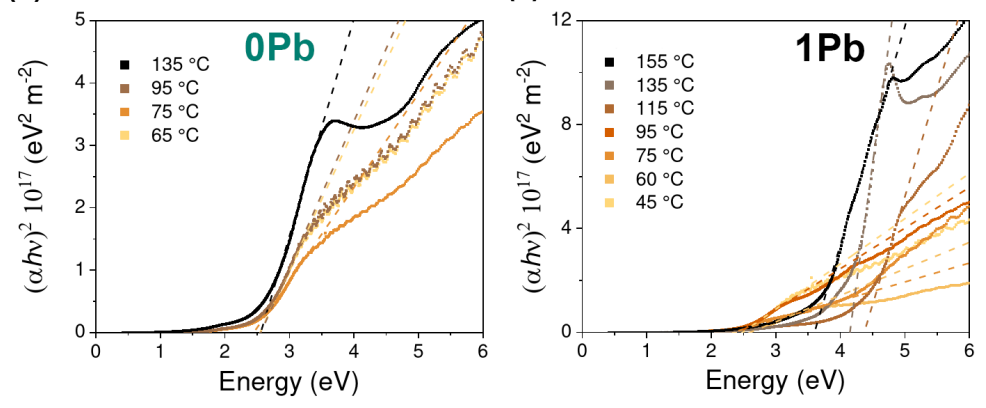

(f)

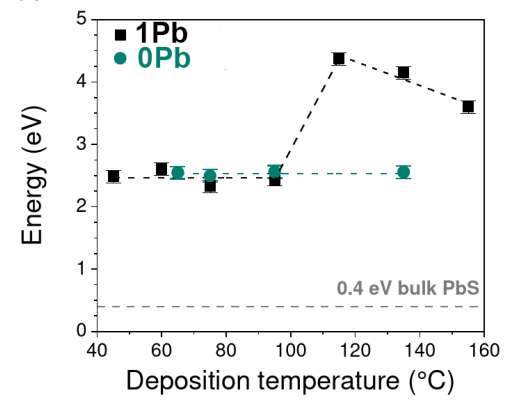

Figure 3.13: Transmittance of ca. $100 \mathrm{~nm}$ thick $\mathrm{PbS}$ films deposited on sapphire with (a) $\mathbf{0 P b}$ and (b) $\mathbf{1 P b}$ at different temperatures. (c) Transmittance of PbS films deposited on sapphire with $\mathbf{1 P b}$ at $60{ }^{\circ} \mathrm{C}$ and different number of deposition cycles. (d) and (e) Tauc plots constructed from (a) and (b) respectively. (f) Optical band gaps of PbS films on sapphire as a function of deposition temperature extracted from Tauc plots in (d) and (b). Dashed grey lines in (a-c) separate wavelength ranges measured with different instruments. All films were deposited with $1 \mathrm{~s}$ precursor pulses and purge durations. 
for $\mathrm{PbS}$ thin films many examples of this approach are present in the literature. ${ }^{156,201-214}$ However, we found optical band gap extraction from Tauc plots to be problematic in the case of $\mathrm{PbS}$. The values extracted from Tauc plots of $\sim 100 \mathrm{~nm}$ thick PbS films deposited at different temperatures were unreasonably large $(\geq 2.5 \mathrm{eV}$, Figure $3.13 \mathrm{~d}-\mathrm{f})$ for the observed grain sizes (Figures 3.8, 3.9, and 3.10). The grains could also be confined in the film thickness direction, which is not visible in top-down SEM. We initially assumed that the grains are columnar and extend through the entire film thickness; however, TEM revealed that this is not the case, and there are also grain boundaries through film thickness (Figure 3.12c)-despite that the grains are still too large to explain the Tauc values.
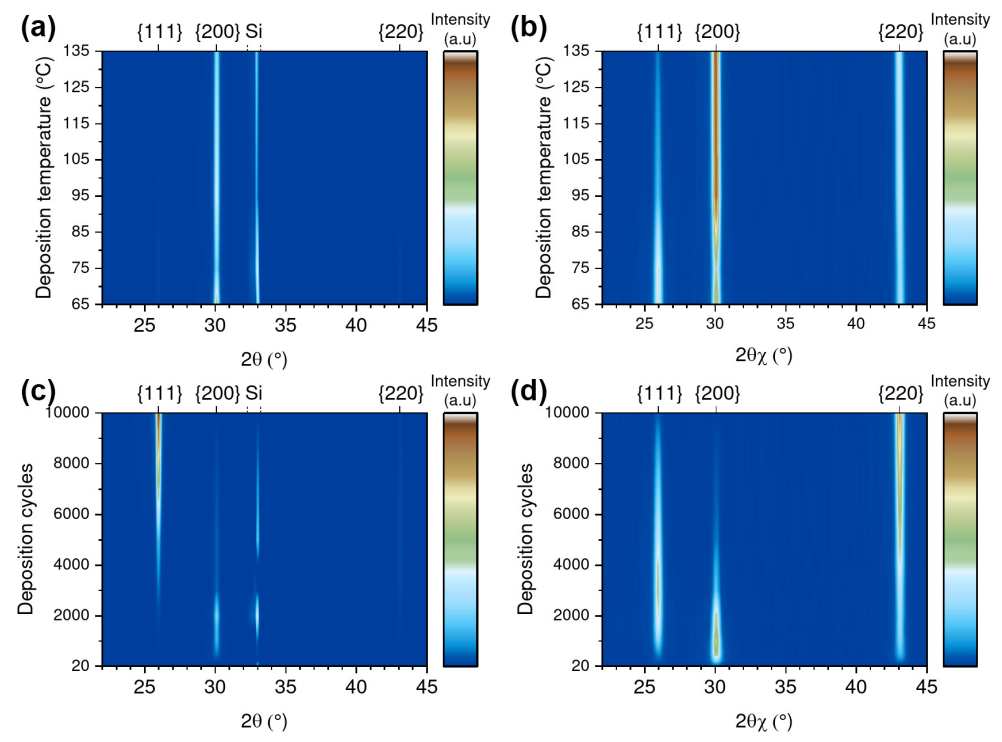

Figure 3.14: Contour profile maps of out of plane (a, c) and in-plane (b, d) XRD data for PbS films deposited with $\mathbf{0 P b}$. In (a, b) films are ca. $100 \mathrm{~nm}$ thick. In (c, d) films were deposited at $75{ }^{\circ} \mathrm{C}$. All films were deposited with $1 \mathrm{~s}$ precursor pulses and purge durations

The grains observed with microscopy methods can consist of smaller crystallites, which in turn if sufficiently small would explain the Tauc values. We calculated crystallite sizes using the Scherrer equation and XRD data measured out-of-plane $(\theta-2 \theta)$ and in-plane $(2 \theta \chi)$, respectively (Figure 3.14), for the films deposited with $\mathbf{0 P b}$. In the $100 \mathbf{n m}$ thick films deposited at different temperatures the in-plane crystallite size increased significantly with increasing deposition temperature, from $47 \pm 3 \mathrm{~nm}$ at $65^{\circ} \mathrm{C}$ to $135 \pm 33 \mathrm{~nm}$ at 

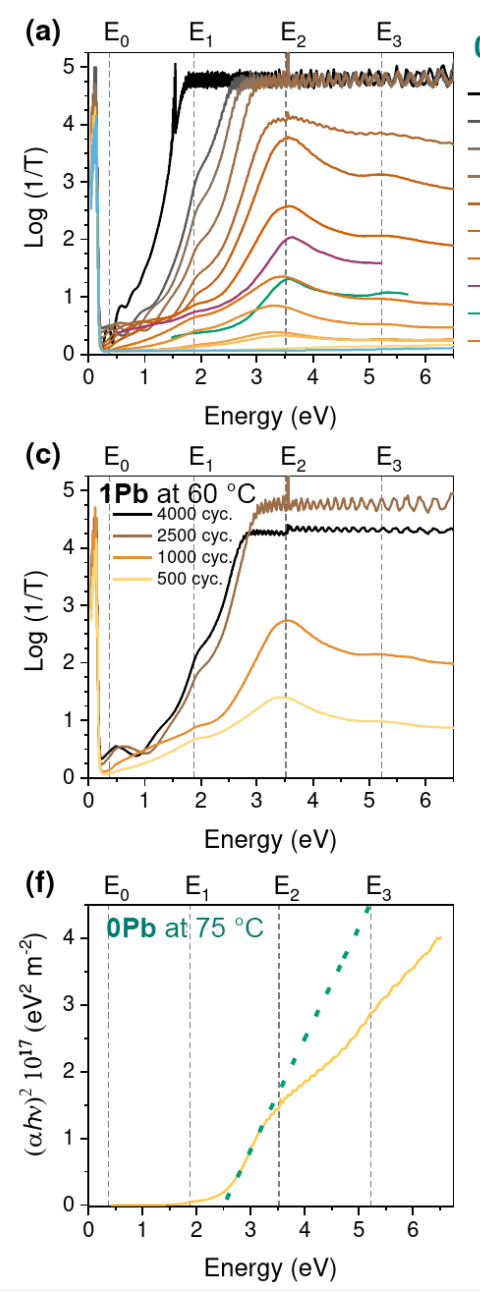

$\mathrm{OPb}$ at $75^{\circ} \mathrm{C}$

10000 cyc. $(430 \mathrm{~nm}) \longrightarrow 250$ cyc. $(12 \mathrm{~nm})$

-5000 cyc. $(216 \mathrm{~nm}) \longrightarrow 100 \mathrm{cyc} .(5 \mathrm{~nm})$

-4000 cyc. $(172 \mathrm{~nm}) \longrightarrow 60$ cyc. $(4 \mathrm{~nm})$

-3000 cyc. $(131 \mathrm{~nm}) \quad 20$ cyc. $(2 \mathrm{~nm})$

2000 cyc. $(86 \mathrm{~nm})$

1500 cyc. $(65 \mathrm{~nm})$

1000 cyc. $(45 \mathrm{~nm})$

- Cardona et al. at $297 \mathrm{~K}(34 \mathrm{~nm})$ Cardona et al. at $77 \mathrm{~K}(34 \mathrm{~nm})$

500 cyc. $(21 \mathrm{~nm})$

(d)

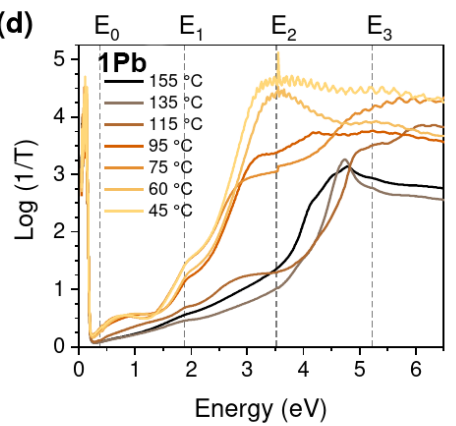

(g)

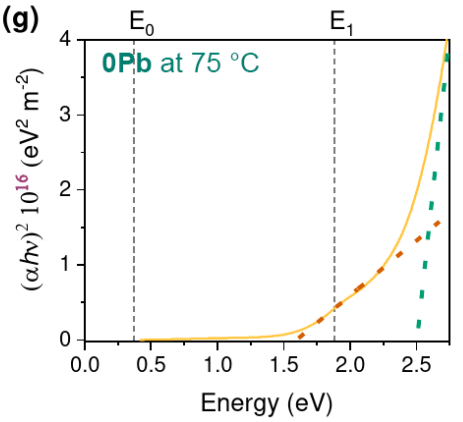

(b)

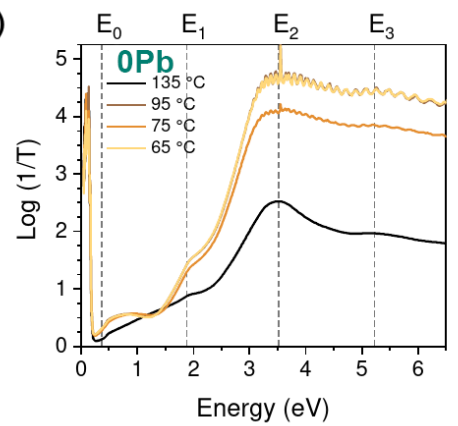

(e)

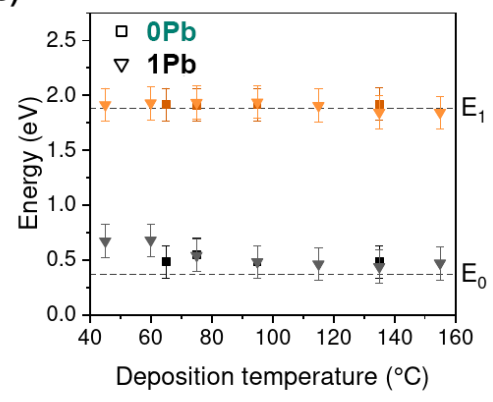

(h)

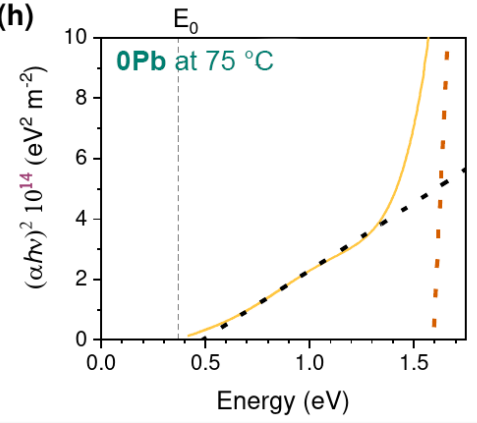

Figure 3.15: (a) $\log (1 / T)$ plots of $\mathrm{PbS}$ films deposited on sapphire with $\mathbf{0 P b}$ at $75{ }^{\circ} \mathrm{C}$ and different number of cycles. (b) $\log (1 / T)$ plots of ca. $100 \mathrm{~nm}$ thick PbS films deposited on sapphire with $\mathbf{0 P b}$ at different temperatures. (c) $\log (1 / T)$ plots of $\mathrm{PbS}$ films deposited on sapphire with $\mathbf{1 P b}$ at $60{ }^{\circ} \mathrm{C}$ and different number of cycles. (d) $\log (1 / T)$ plots of ca. $100 \mathrm{~nm}$ thick PbS films deposited on sapphire with $\mathbf{1 P b}$ at different temperatures. (e) Interband transitions energies obtained from $\log (1 / T)$ plots in (b) and (d) as a functiondeposition temperature. (f-h) Tauc plot of ca. $100 \mathrm{~nm}$ $\mathrm{PbS}$ film deposited on sapphire with $\mathbf{0 P b}$ at $75{ }^{\circ} \mathrm{C}$. Note that changing the examined energy range and Tauc property scale $\left[(\alpha h v)^{2}\right.$, y-axis] allows fitting three different energies ca. $0.5 \mathrm{eV}, 1.6$ and $2.5 \mathrm{eV}$ which correspond to $E_{0}, E_{1}$ and $E_{2}$ transitions respectively. In the analogous $\log (1 / T)$ plot, see for example (b), features corresponding to these transitions are immediately visible without extensive rescaling. In all figures dashed gray lines are reference energy values by Cardona and Greenaway. ${ }^{200}$ All films were deposited with $1 \mathrm{~s}$ pulse and purge durations. 
$135^{\circ} \mathrm{C}$, whereas the out-of-plane crystallite size remained almost unchanged at ca. $56 \mathrm{~nm}$. The dependence of the in-plane crystallite size on the deposition temperature is in good agreement with the observed morphology (Figure 3.8) and trends in electrical properties (Figure 3.11). The out-of-plane XRD patterns show that in these films most crystallites are 200 oriented with some minor 111 and 220 components. Correspondingly, the most prominent reflections in the in-plane patterns of these films are also 200 (Figure 3.14c). In the films deposited at $75{ }^{\circ} \mathrm{C}$ with different number of cycles both the out-of-plane and in-plane crystallite sizes increase rapidly with the increasing number of cycles until 1500 cycles (Figure 3.12d). After 1500 cycles, the growth of the in-plane crystallite size slows down considerably, whereas the growth of the out-of-plane crystallite size continues and approaches the limit that can be measured from peak broadening. The XRD patterns also reveal changes in the film texture. In the out-of-plane direction 200 dominates up to 2000 cycles and 111 afterward, whereas in the in-plane direction also 200 dominates up to 2000 cycles, then 111 at 3000 cycles, and 220 afterward. The crystallite dimensions we observed imply no quantum confinement effects in the films deposited with 500 cycles or more and are therefore in disagreement with the Tauc values. Others have also noted a similar discrepancy between the Tauc values and crystallite sizes but provided no explanation. ${ }^{201,202}$ Additionally, there is an inconsistency between the reported Tauc values and electronic band gaps. Dasgupta et al. determined the electronic band gap of ALD PbS films using scanning tunneling microscopy (STM). ${ }^{35}$ These measurements showed that the electronic band gap decays non-linearly with increasing film thickness and approaches the bulk value when the film thickness is $16 \mathrm{~nm}$, which is in line with the exciton Bohr radius of $\mathrm{PbS}$ (ca. $20 \mathrm{~nm})$.

The cause of the conflicting evidence is elucidated in the earlier works of Gibson, ${ }^{215,216}$ Scanlon, ${ }^{217}$ and Cardona and Greenaway. ${ }^{200}$ on the optical and electronic properties of $\mathrm{PbS}$. Examination of $\mathrm{PbS}$ transmittance or reflectance in a wide energy range $(0.2-20 \mathrm{eV})$ reveals several band-to-band transitions in addition to the lowest energy transition, i.e., 
the band gap or $E_{0}$. Cardona and Greenaway give a comprehensive list of these transitions $\left(E_{0}\right.$ to $\left.E_{6}\right)$ as well as estimate some of the energies of these transitions using reflectance and transmittance spectra from single crystals and epitaxial thin films. In the case of thin film transmittance spectra, the transition energies were estimated based on the position of the features in the $\log (1 / T)$ plot. In fact, $\log (1 / T)$ plots of our films are strikingly similar to Cardona and Greenaway (Figure 3.15a-d) and contain four features corresponding to the interband transitions $E_{0}-E_{3}$. The optical band gap of thicker films ( $>500$ deposition cycles) defined from $\log (1 / T)$ plots is ca. $0.5 \mathrm{eV}$ (Figure 3.12e and Figure 3.15e). This value is in excellent agreement with the reported bulk band gap and electronic band gap of $\mathrm{PbS}$, whereas the increase of the interband transition energies in very thin films $(<500$ deposition cycles) is in excellent agreement with decrease of crystallite size below the exciton radius $(20 \mathrm{~nm}$ ) in these films (Figure 3.12d,e).

To summarize, a Tauc plot is not an ideal method for determining the optical band gap of $\mathrm{PbS}$. The guidelines available in the literature suggest deriving the band gap from the most noticeable Tauc property $(\alpha h v)^{2}$ change, ${ }^{218}$ which in our case yielded unreasonably large values. However, in optical spectra the most noticeable feature does not necessarily correspond to the band gap $\left(E_{0}\right)$. For PbS the most noticeable feature is $E_{2}$ (Figures 3.13d,e and 3.15a-d,f). ${ }^{200}$ The Tauc property itself provides an additional challenge for narrow gap materials as it accentuates higher energy transitions and masks lower energy transitions (Figure 3.15f-h). Logarithmic plots of Tauc property can reveal these hidden transitions and allow extraction of their energies. In addition to $E_{2}$, we were able to find also $E_{0}$ (ca. $0.5 \mathrm{eV}$ ) and $E_{1}$ (ca. $1.6 \mathrm{eV}$ ) by extensive rescaling (Figure 3.15g,h). An accurate distinction of the band gap transition from other higher energy interband transitions from optical data requires consideration of a wide energy range that covers the region of the bulk band gap of $\mathrm{PbS}(0.2-1.0 \mathrm{eV})$. In our case $\log (1 / T)$ plots were more robust than the Tauc plots for this purpose. The transitions observed in optical data are best understood in terms of the band structure of $\mathrm{PbS}$, for which we refer the reader to Albanesi et al. ${ }^{219}$ 


\subsubsection{Deposition of PbS onto Halide Perovskites}

We investigated whether the ALD processes developed and described here are compatible with halide perovskites. When sulfides are deposited onto perovskites, there is a possibility of an anion exchange between the perovskite and $\mathrm{H}_{2} \mathrm{~S}$ (Equation 3.1).

$$
\mathrm{CH}_{3} \mathrm{NH}_{3} \mathrm{PbI}_{3}(\mathrm{~s})+\mathrm{H}_{2} \mathrm{~S}(\mathrm{~g}) \longrightarrow \mathrm{PbS}(\mathrm{s})+\mathrm{CH}_{3} \mathrm{NH}_{3} \mathrm{I}(\mathrm{g}) \uparrow+\mathrm{HI}(\mathrm{g}) \uparrow
$$

However, based on thermodynamic values reported in the literature, ${ }^{189,220,221}$ this reaction is endothermic $\left(\Delta H^{\star}=+146 \mathrm{~kJ} / \mathrm{mol}\right)$ and unfavorable $\left(\Delta G^{*}=+121 \mathrm{~kJ} / \mathrm{mol}\right)$. In addition, it was experimentally verified that the MAPI film was unaffected when $\mathrm{H}_{2} \mathrm{~S}$ was pulsed onto it at $50{ }^{\circ} \mathrm{C}$ (Figure 3.17). We deposited $\mathrm{PbS}$ films at different temperatures on MAPI films (Figure 3.16a) and used XRD to examine the state of the MAPI film after the deposition. In the case of the $\mathbf{0} \mathbf{P b}-\mathrm{H}_{2} \mathrm{~S}$ process, only the lowest deposition temperature of $65{ }^{\circ} \mathrm{C}$ left the MAPI film unchanged (Figure 3.16b and Figure 3.18). At deposition temperatures higher than $65^{\circ} \mathrm{C} \mathrm{PbI}_{2}$ reflections appeared and increased in intensity, whereas MAPI reflections decreased in intensity. This is caused by the decomposition of MAPI into $\mathrm{PbI}_{2}$ and volatile $\mathrm{CH}_{3} \mathrm{NH}_{3} \mathrm{I}$ upon heating to the target deposition temperature before $\mathrm{PbS}$ deposition begins (Figure 3.16e). When the $\mathrm{PbS}$ deposition temperature was above $95{ }^{\circ} \mathrm{C}$, also the intensity of $\mathrm{PbI}_{2}$ reflections decreased. This decrease is caused by the sublimation of $\mathrm{PbI}_{2}$ before the $\mathrm{PbS}$ deposition.

MAPI films remained unchanged when $\mathrm{PbS}$ was deposited on them with $\mathbf{1 P b}$ in the temperature range $45-60{ }^{\circ} \mathrm{C}$ (Figure $3.16 \mathrm{c}$ and Figure 3.18). At the deposition temperature of $75{ }^{\circ} \mathrm{C}$ and higher, MAPI expectedly decomposed into $\mathrm{CH}_{3} \mathrm{NH}_{3} \mathrm{I}$ and $\mathrm{PbI}_{2}$, as in the case when $\mathbf{0 P b}$ was used. At the deposition temperature of $95{ }^{\circ} \mathrm{C}$ and higher reflections of $\mathrm{Pb}_{5} \mathrm{~S}_{2} \mathrm{I}_{6}$ and $\mathrm{Pb}_{3}\left(\mathrm{CO}_{3}\right)_{2}(\mathrm{OH})_{2}$ phases appeared (Figure 3.18). The appearance of these phases coincides with the onset of formation of elemental Pb. Notably, no $\mathrm{Pb}_{5} \mathrm{~S}_{2} \mathrm{I}_{6}$ was observed with $\mathbf{0 P b}$. These results imply that the species involved in the formation of ele- 

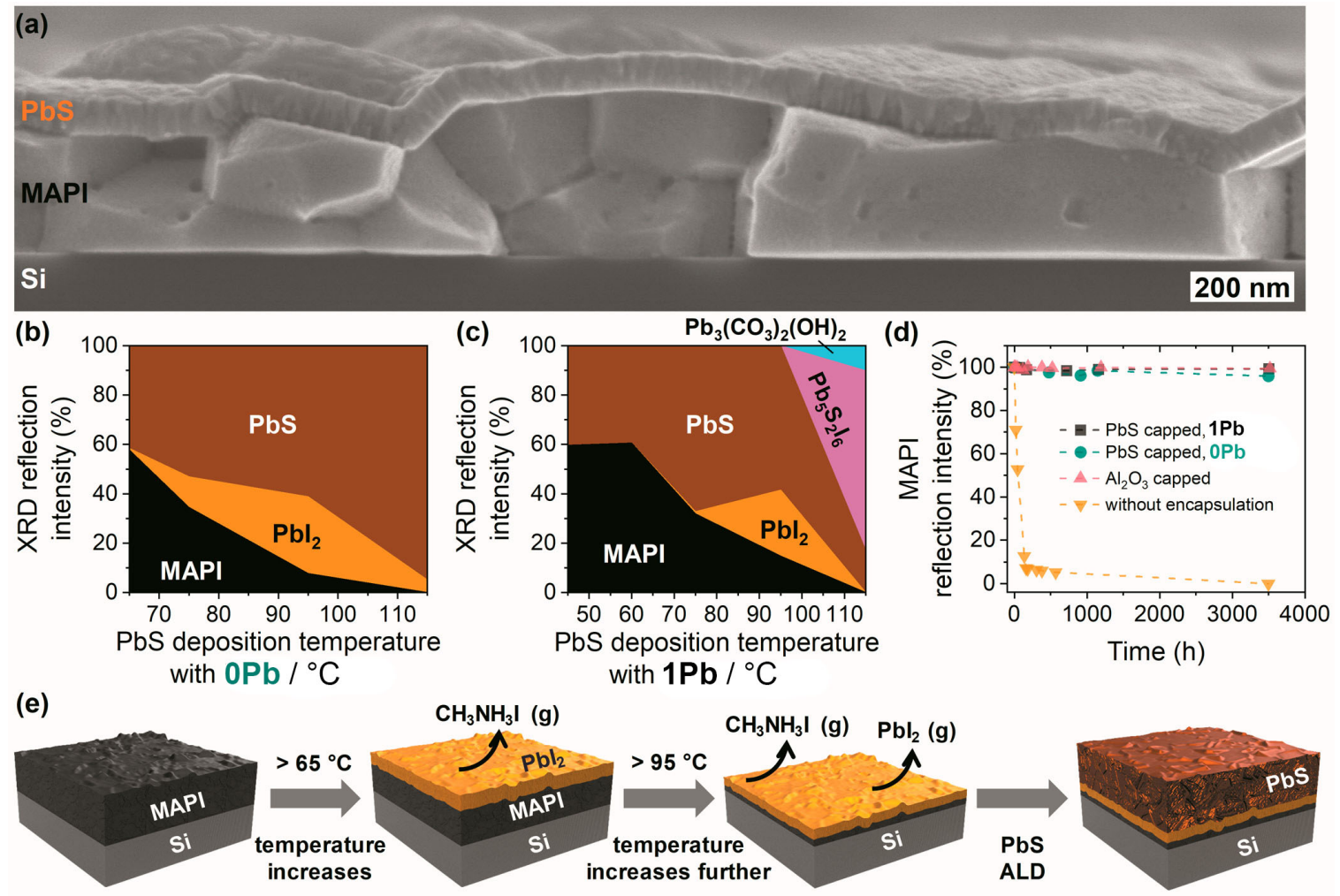

Figure 3.16: (a) Cross-section SEM image of a PbS-capped MAPI used for stability study in (d). $\mathrm{PbS}$ was deposited with $\mathbf{1 P b}$. To examine the cross section, the sample was broken in half after being stored in air for 413 days. For EDS line scans across the cross section see Figure 3.17. Relative reflection intensities (area) of crystalline phases observed in GIXRD patterns of samples where PbS was deposited on MAPI films with (b) $\mathbf{0 P b}$ and (c) $\mathbf{1} \mathbf{P b}$ at different temperatures. (b) and (c) are derived from GIXRD patterns in Figure 3.18. MAPI film reflection intensity (d) as a function of storage time in ambient air (open system) with and without different capping films derived from GIXRD patterns in Figures 3.19-3.20. PbS capping film was deposited at $45^{\circ} \mathrm{C}$ with $\mathbf{1 P b}$ and at $65^{\circ} \mathrm{C}$ with $\mathbf{0 P b}$. The $\mathrm{Al}_{2} \mathrm{O}_{3}$ capping film was deposited at $65^{\circ} \mathrm{C}$ with 800 cycles of the TMA- $\mathrm{H}_{2} \mathrm{O}$ process using a $1 \mathrm{~s}$ precursor pulse and $4.0 \mathrm{~s}$ purge durations. The capping film thicknesses were 66,50 , and $52 \mathrm{~nm}$ for $\mathrm{PbS}$ deposited with $\mathbf{1} \mathbf{P b}, \mathrm{PbS}$ deposited with $\mathbf{0 P b}$, and $\mathrm{Al}_{2} \mathrm{O}_{3}$, respectively. $\mathrm{PbS}$ films in (b), (c), and (d) were deposited with 1000 cycles, $1 \mathrm{~s}$ precursor pulses, and purge durations. (e) Schematic of the changes occurring on the MAPI film in the ALD reactor when deposition temperatures higher than $65^{\circ} \mathrm{C}$ are used. 

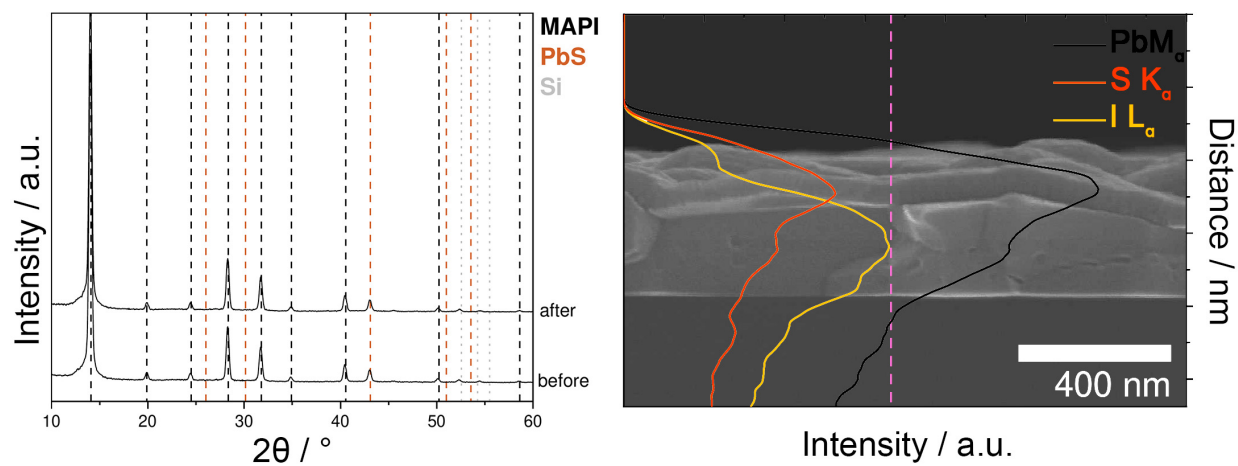

Figure 3.17: (left) GIXRD pattern of a $\mathrm{CH}_{3} \mathrm{NH}_{3} \mathrm{PbI}_{3}$ (MAPI) film before and after 1000 cycles of $1 \mathrm{~s}$ long $\mathrm{H}_{2} \mathrm{~S}$ pulses separated by $3.0 \mathrm{~s}$ long purges at $50{ }^{\circ} \mathrm{C}$. (right) Cross-section SEM image of a $\mathrm{PbS}$ capped MAPI and EDS line scans along the purple line. PbS was deposited with $\mathbf{1 P b}$ at $45^{\circ} \mathrm{C}$ with 1000 cycles of $1 \mathrm{~s}$ long pulse and purge durations. To examine the cross section the sample was broken in half after being stored in air for 413 days. The EDS line scan data was smoothed (adjacent averaging) for clarity. Note also the partial overlap between $\mathrm{Pb} \mathrm{M} \alpha$ and $\mathrm{S} \mathrm{K} \alpha$ lines.
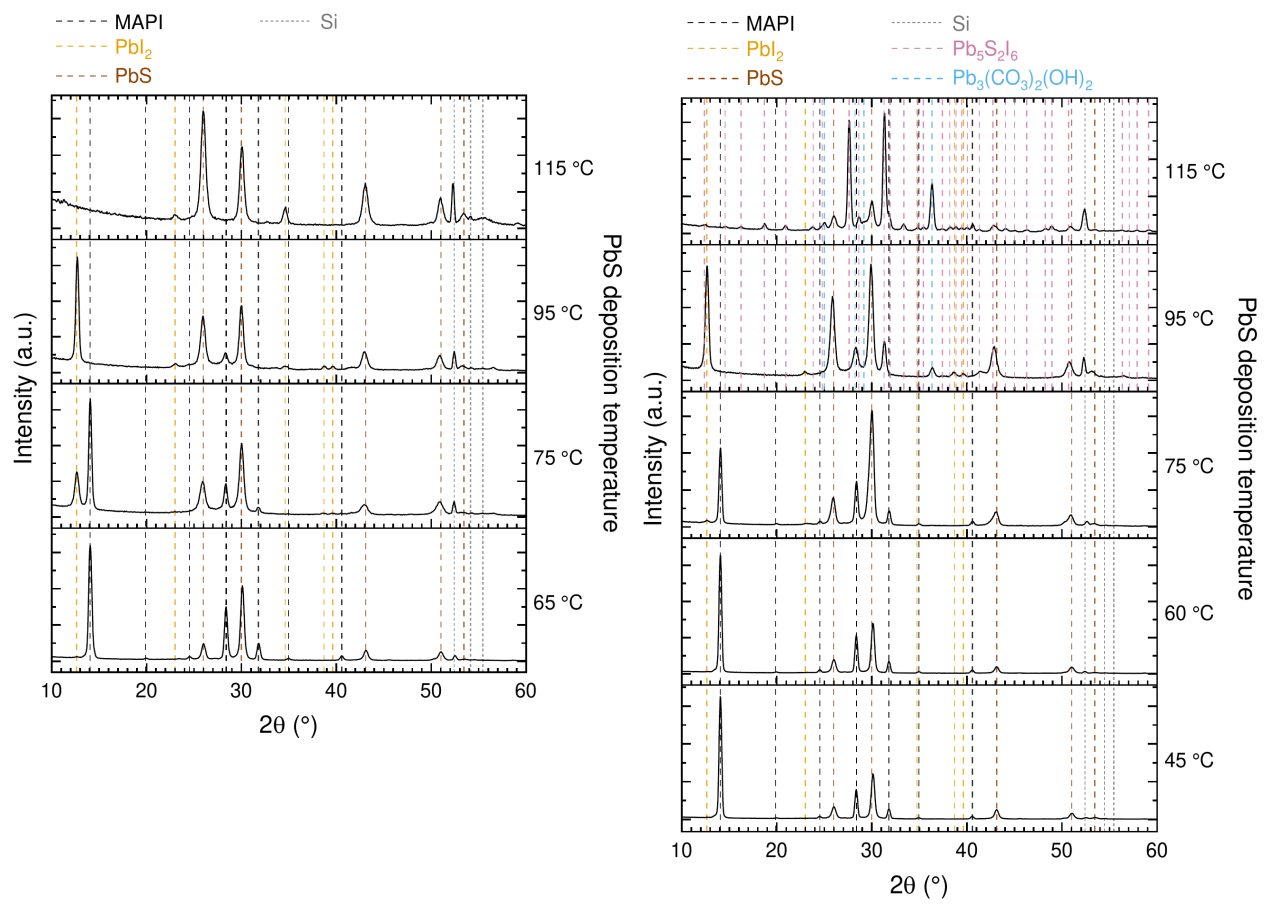

Figure 3.18: (left) GIXRD patterns of samples where $\mathrm{PbS}$ was deposited with $\mathbf{0 P b}$ at different temperatures on MAPI films on silicon substrates. PbS was deposited with 1000 cycles, 1 s precursor pulses and purge durations. (right) GIXRD patterns of samples where $\mathrm{PbS}$ was deposited with $\mathbf{1 P b}$ at different temperatures on MAPI $\left(\mathrm{CH}_{3} \mathrm{NH}_{3} \mathrm{PbI}_{3}\right)$ films on silicon substrates. $\mathrm{PbS}$ was deposited with 1000 cycles, $1 \mathrm{~s}$ precursor pulses and purge durations. 
mental $\mathrm{Pb}$ can react with the $\mathrm{PbI}_{2}$ remaining from the decomposition of the MAPI film to yield $\mathrm{Pb}_{5} \mathrm{~S}_{2} \mathrm{I}_{6}$. The $\mathrm{Pb}_{3}\left(\mathrm{CO}_{3}\right)_{2}(\mathrm{OH})_{2}$ phase is apparently formed upon exposure of $\mathrm{Pb}_{5} \mathrm{~S}_{2} \mathrm{I}_{6}$ or $\mathrm{Pb}$ to the ambient air.

In the case of $\mathrm{PbS}$ deposition on MAPI the $\mathbf{1} \mathbf{P b}-\mathrm{H}_{2} \mathrm{~S}$ process is superior to the $\mathbf{0 P b}$ $\mathrm{H}_{2} \mathrm{~S}$. 1Pb offers a $20{ }^{\circ} \mathrm{C}$ wide temperature window where $\mathrm{PbS}$ can be deposited on MAPI, whereas $\mathbf{0 P b}$ only provides a single useful deposition temperature of $65^{\circ} \mathrm{C}$. The benefit of the wider temperature window comes with the cost of more complicated synthesis. Therefore, $\mathbf{0 P b}$ can be a more convenient choice for perovskites that can withstand higher deposition temperatures such as $\mathrm{FA}_{x} \mathrm{MA}_{1-x} \mathrm{~Pb}\left(\mathrm{Br}_{y} \mathrm{I}_{1-y}\right)_{3}$ (up to $\left.120{ }^{\circ} \mathrm{C}\right){ }^{222} \mathrm{Cs}_{x} \mathrm{FA}_{1-x} \mathrm{~Pb}\left(\mathrm{Br}_{y} \mathrm{I}_{1-y}\right)_{3}$ (up to $110{ }^{\circ} \mathrm{C}$ ), ${ }^{223}$ and others. ${ }^{173}$ On the contrary, the deposition of Pb makes 1Pb less relevant at higher deposition temperatures. Ultimately, the need of certain electrical properties can dictate which of the two processes is more suitable in a specific device design.

\subsubsection{Application of PbS as a Protective Layer}

Finally, to determine whether encapsulation with an ALD PbS film can protect halide perovskites from ambient air, we followed the state of PbScapped MAPI films with XRD. Without any encapsulation, the MAPI film completely decomposed into $\mathrm{PbI}_{2}$ in a week, and after 5 months, $\mathrm{PbI}_{2}$ had further reacted into $\mathrm{PbI}(\mathrm{OH})$ (Figure 3.16d and Figure 3.19). On the other hand, MAPI films were stable for at least 5 months in air when encapsulated with $\mathrm{PbS}$ films deposited either from $\mathbf{0 P b}$ or $\mathbf{1 P b}$ (Figure 3.20). For comparison, we also evaluated the stability of a MAPI film encapsulated with ALD $\mathrm{Al}_{2} \mathrm{O}_{3}$ film (Figure 3.20). Thus the improve-

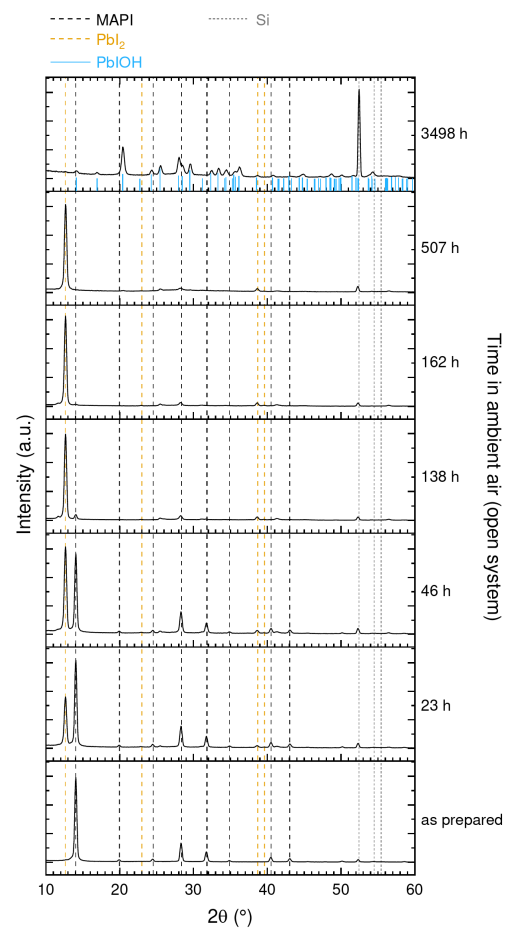

Figure 3.19: GIXRD of an unprotected MAPI film stored in ambient air. 
ment in the MAPI stability provided by the PbS encapsulation equals that provided by an $\mathrm{Al}_{2} \mathrm{O}_{3}$ film of similar thickness (Figure 3.16c).

We attempted to further characterize the barrier properties of our films by depositing them on $50 \mu \mathrm{m}$ thick biaxially-oriented poly(ethylene terephthalate) (BoPET) and measuring water vapour (WVTR) and oxygen transmission rates (OTR) of these samples. However, film deposition on BoPET was challenging. Relatively long pulse (6 s) and purge times (3 s) were required for visually uniform films on BoPET, and the GPC was significantly lower than on rigid substrates: $0.20 \AA$ with $\mathbf{0 P b}$ at $65{ }^{\circ} \mathrm{C}$ and $0.25 \AA$ with $\mathbf{1 P b}$ at $45{ }^{\circ} \mathrm{C}$. With the PbS capping the OTR decreased by ca. $90 \%$ with respect to the bare BoPET, however, the WVTR decreased only slightly (Figure 3.21). We also observed a significant decrease in the OTR and only a slight decrease in the WVTR of the reference BoPET samples coated with $\mathrm{ALD} \mathrm{Al}_{2} \mathrm{O}_{3}$. These results indicate that $\mathrm{ALD} \mathrm{PbS}$ and $\mathrm{Al}_{2} \mathrm{O}_{3}$ coatings perform similarly in these barrier tests, despite the polycrystalline structure of the $\mathrm{PbS}$. In general, it is thought that amorphous films will serve as better barriers because they lack grain boundaries along which diffusion can be faster. ${ }^{224}$ A thin amorphous passivating $\mathrm{PbSO}_{3} / \mathrm{PbSO}_{4}$ layer that forms on the $\mathrm{PbS}$ surface ${ }^{225}$ can account for the $\mathrm{Al}_{2} \mathrm{O}_{3}$ comparable barrier performance of our crystalline $\mathrm{PbS}$ films.

While low deposition temperatures enable the use of polymers as substrates, the ALD of barrier films on polymers is not straightforward. This is partially because of the engineering challenges associated with the handling of flexible substrates but even more because of the complex mechanism of ALD film nucleation and growth on polymers. ${ }^{226}$ Barrier performance of an ALD film is affected by many factors such as porosity, roughness, and surface structure of the substrate polymer as well as crystallinity, impurity content, and thickness of the coating itself. ${ }^{227}$ For instance, a thicker coating could block gases more efficiently and be more mechanically durable but could also crack more easily, whereas a thinner coating would not necessarily form a continuous and tight layer. ${ }^{226-228}$ In the literature WVTR values as low as 10-1 g m-2 day-1 were reported for $50 \mathrm{~nm}$ thick 


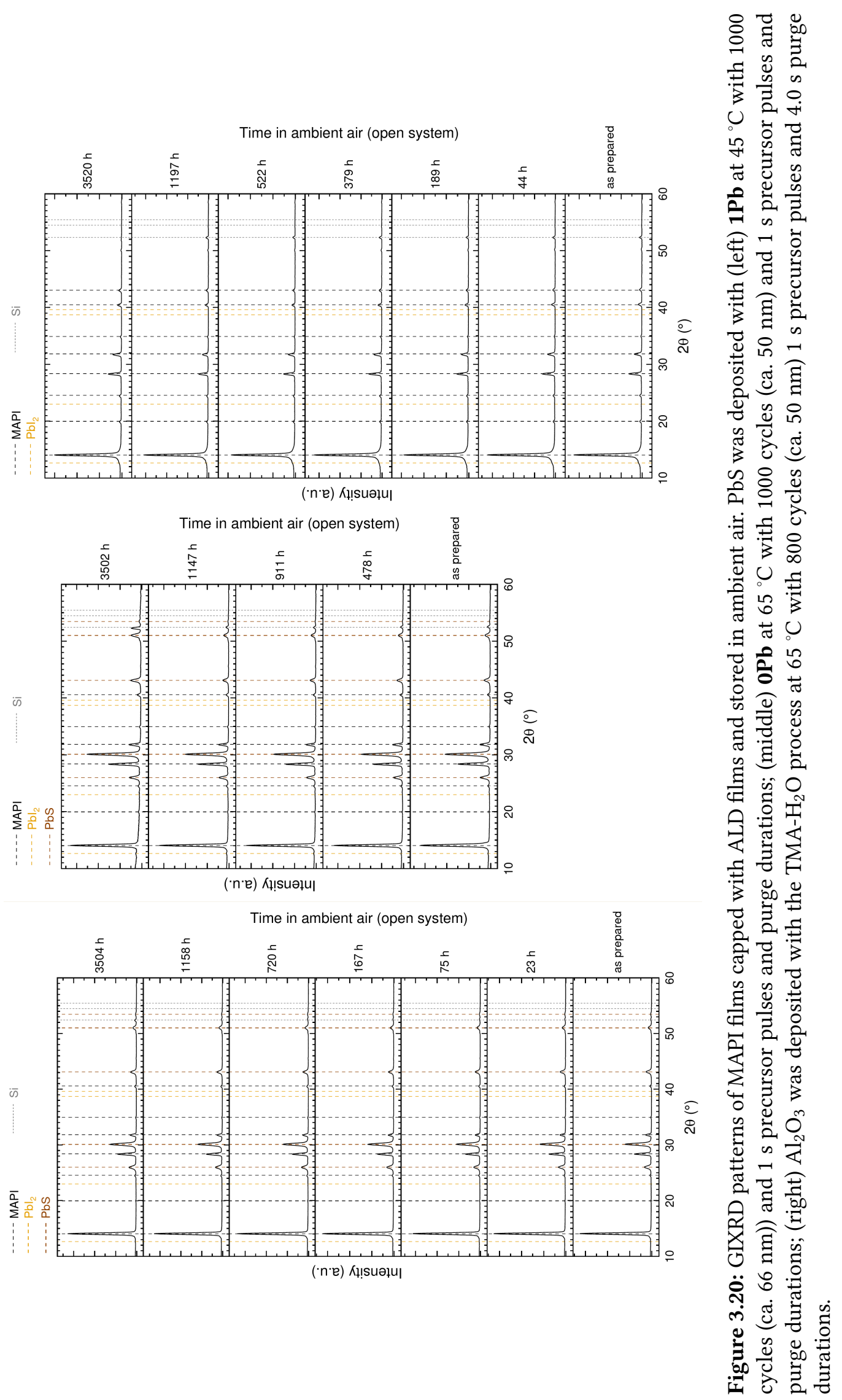


(a)

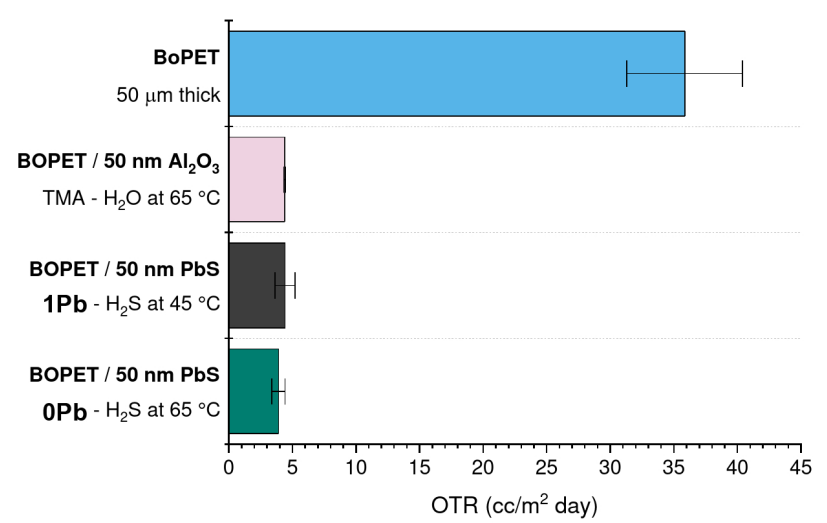

(b)

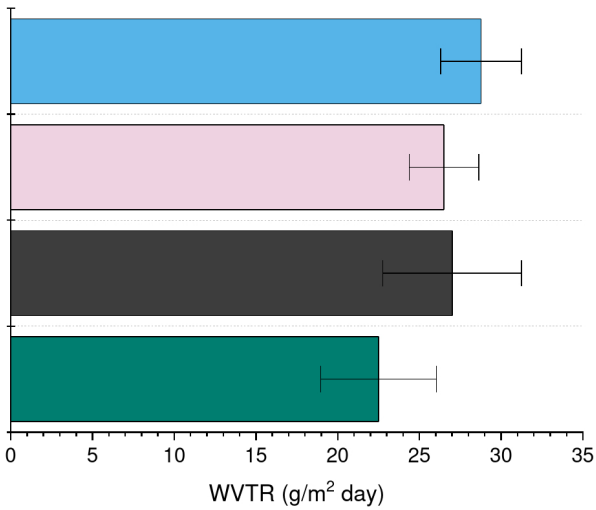

Figure 3.21: (top) (a) Oxygen transmission rates and (b) water vapour transmission rates for bare BoPET and BoPET with an ALD film deposited on it. $\mathrm{Al}_{2} \mathrm{O}_{3}$ films were deposited with $1 \mathrm{~s}$ precursor pulses and $4.0 \mathrm{~s}$ purge durations. PbS films were deposited with $6.0 \mathrm{~s}$ precursor pulses and $3.0 \mathrm{~s}$ purge durations.

thermal ALD $\mathrm{Al}_{2} \mathrm{O}_{3}$ films deposited at $65{ }^{\circ} \mathrm{C}$ on thicker $(125 \mu \mathrm{m})$ PET substrates ${ }^{229}$ and even lower ones for $\mathrm{ALD} \mathrm{Al}_{2} \mathrm{O}_{3}$ on rigid substrates measured with electrical calcium corrosion tests. ${ }^{230}$ On the basis of the literature results and our own observations, we anticipate that further optimization of the pretreatment, process, and handling parameters will enable lower WVTR values for ALD PbS coatings on polymers. However, the extent of optimizing these parameters is a topic of a dedicated study and outside of the scope of this work which aimed to demonstrate the ability of ALD PbS films to protect halide perovskite films on rigid substrates.

\subsection{Conclusions}

We described two new ALD processes for deposition of PbS films using $\mathbf{0 P b}, \mathbf{1 P b}$, and $\mathrm{H}_{2} \mathrm{~S}$ as precursors. The scale-up of $\mathbf{1 P b}$ synthesis afforded reasonable precursor purity and quantity for process development. Yet challenges encountered during the scale-up highlight the importance of further studies on the synthetic chemistry of volatile $\mathrm{Pb}$ complexes. Both $\mathrm{Pb}$ precursors enable deposition of high quality $\mathrm{PbS}$ films at low temperatures of $65-155^{\circ} \mathrm{C}$ with $\mathbf{0 P b}$ and $45-75^{\circ} \mathrm{C}$ with $\mathbf{1 P b}$. The $\mathrm{PbS}$ films are uniform, crystalline, 
and of high purity as well as conductive and $p$-type. We demonstrated that at deposition temperatures of $\leq 65{ }^{\circ} \mathrm{C}$ these ALD processes are compatible with the perovskite halide $\left(\mathrm{CH}_{3} \mathrm{NH}_{3} \mathrm{PbI}_{3}\right)$ films. $\mathbf{1} \mathbf{P b}$ is more suitable for capping the $\mathrm{CH}_{3} \mathrm{NH}_{3} \mathrm{PbI}_{3}$ perovskite because it is applicable in a range of temperatures versus only one temperature with $\mathbf{0 P b}$. $\mathbf{0 P b}$, on the other hand, is a more suitable choice for perovskites that withstand higher temperatures such as those based on cesium or formamidinium as well as in cases where high carrier mobility is sought. Capping the $\mathrm{CH}_{3} \mathrm{NH}_{3} \mathrm{PbI}_{3}$ films with ALD PbS prevents the perovskite from decomposing in the ambient air. The encapsulation performance of ALD PbS is comparable to $\mathrm{ALD} \mathrm{Al}_{2} \mathrm{O}_{3}$. Compatibility with various substrates such as polymers provided by the low deposition temperatures and the ability to control electrical and optical properties of the $\mathrm{PbS}$ films offer flexibility for design of device structures based on halide perovskites or $\mathrm{PbS}$. We hope that this work will encourage other studies on the use of non-oxide ALD films together with halide perovskites and that the new ALD PbS processes are viable options for PbS based devices.

\subsection{Experimental Details}

\subsubsection{Precursor Synthesis}

Lead(II) bis[bis(trimethylsilyl) amide] (0Pb) was synthesized from lead(II) chloride (Alfa Aesar, 99.999\%) and lithium bis(trimethylsilyl)amide $\left(\mathrm{LiN}\left(\mathrm{SiMe}_{3}\right)_{2}\right)$, Aldrich, 95\%) as previously described. ${ }^{34,231}$ Lead(II) rac- $\mathrm{N}^{2}, \mathrm{~N}^{3}$-di-tert-butylbutane-2,3-diamide (1Pb) was prepared by a modified literature procedure. ${ }^{182} \mathbf{0 P b}(13.160 \mathrm{~g}, 24.923 \mathrm{mmol})$ was dissolved in $100 \mathrm{~mL}$ of toluene at ambient temperature under nitrogen in a $200 \mathrm{~mL}$ Schlenk flask to yield a clear orange solution. Then, free rac- $N, N^{\prime}$-di-tert-butyl-2,3-diaminobutane ${ }^{182}$ (5.000 g, $24.94 \mathrm{mmol}$ ) diluted in $10 \mathrm{~mL}$ of toluene was added all at once via cannula. Some decomposition was evident as the mixture turned dark green-gray with the precipitation of metallic $\mathrm{Pb}$. The mixture was then immediately heated to reflux by placing in 
a preheated oil bath to facilitate the transamination reaction. Aliquots were taken by syringe and placed into an NMR tube under nitrogen; the majority of solvent was removed under vacuum; then the residue was dissolved in $\mathrm{C}_{6} \mathrm{D}_{6}$. The reaction was monitored by ex situ ${ }^{1} \mathrm{H}$ NMR spectroscopy until the signal for $\mathbf{0 P b}$ had disappeared (ca. $3 \mathrm{~h}$ ). The reaction mixture was then cooled to ambient temperature, and volatiles (i.e., free bis(trimethylsilyl)amine and toluene) were removed under high vacuum (ca. 10 mTorr) until a blood-red oil remained. Most of the unreacted free diamine ligand was removed by distillation through a $90^{\circ}$ glass elbow under high vacuum $\left(40{ }^{\circ} \mathrm{C} / 10-2\right.$ Torr $)$ into a dry ice-acetone cooled $\left(-78{ }^{\circ} \mathrm{C}\right)$ receiving Schlenk flask as a bright red liquid, contaminated by a small amount of $\mathbf{1 P b}$. Removal of free ligand was conveniently indicated by solidification of the mixture in the pot. After the receiving flask was replaced with a clean Schlenk flask, the crude product was sublimated $\left(60{ }^{\circ} \mathrm{C} / 10 \mathrm{mTorr}\right)$ into the air-cooled elbow to yield $\mathbf{1 P b}(5.1 \mathrm{~g}, 51 \%$, based on $\mathbf{0 P b})$ as a blood-red crystalline solid. Yield was reduced as $\mathbf{1 P b}$ saturated the air-cooled condenser, and separation of the purified product followed by another sublimation of the remaining crude can improve yields. Some liquid condensed in the receiving flask and was removed mechanically. Samples prepared in this way had identical spectroscopic and physical properties to those previously reported. ${ }^{182}$

\subsubsection{Film Deposition}

Caution! $\mathrm{H}_{2} \mathrm{~S}$ is a flammable, corrosive, and highly toxic gas. The ALD reactor used in this work was in a dedicated laboratory equipped with an $\mathrm{H}_{2} \mathrm{~S}$ sensor and where gas masks were readily available. The $\mathrm{H}_{2} \mathrm{~S}$ bottle was kept in a gas cabinet with isolated ventilation. EPDM (ethylene propylene diene monomer) rubber seals were used. Exhaust was bubbled though an aqueous solution of $\mathrm{Cu}\left(\mathrm{NO}_{3}\right)_{2}$. The authors urge the readers to acquaint themselves with the article by Dasgupta et al. ${ }^{232}$ before working with $\mathrm{H}_{2} \mathrm{~S}$.

The films were deposited in a commercial, hot-wall, flow-type F120 ALD reactor (ASM Microchemistry Ltd.) operated under reduced pressure of ca. 10 mbar (ca. 7.5 Torr). A con- 
tinuous flow (400 sccm) of nitrogen (AGA, 99.999\%), introduced into the reactor through a gas purifier (SAES Pure Gas, MC1-902F, $\mathrm{H}_{2} \mathrm{O}, \mathrm{O}_{2}, \mathrm{CO}, \mathrm{CO}_{2}, \mathrm{H}_{2}, \mathrm{NMHC}$ removal <1 ppb), was used as a carrier and purge gas. $\mathbf{0 P b}$ and $\mathbf{1 P b}$ were sublimed from open glass boats held inside the reactor at 60 and $40{ }^{\circ} \mathrm{C}$, respectively. For both precursors the first signs of decomposition were observed at deposition temperatures of $155^{\circ} \mathrm{C} . \mathrm{H}_{2} \mathrm{~S}$ (Linde, 99.5\%) was introduced into the reactor through a gas purifier (SAES Pure Gas, MC1-302F, $\mathrm{H}_{2} \mathrm{O}$ removal $<1 \mathrm{ppb}$ ) and a needle valve, at a flow rate of $14 \mathrm{sccm} . \mathrm{Al}_{2} \mathrm{O}_{3}$ was deposited by using the trimethylaluminum (TMA)- $\mathrm{H}_{2} \mathrm{O}$ process. The films were deposited on $5 \times 5 \mathrm{~cm}^{2}$ silicon (100), soda lime glass, and biaxially-oriented poly(ethylene terephthalate) (BoPET, GoodFellow, $50 \mu \mathrm{m}$ thick) substrates as well as on 2 in. sapphire wafers (c-plane off to $\mathrm{M}$ plane by $0.2 \pm 0.1^{\circ}$, double side polished, University Wafer). For the deposition on BoPET a special adapter was machined from $\mathrm{Al}$ sheets to prevent the movement of the polymer substrate by pressing it at the edges, without the adapter the movement of the polymer occasionally blocked the gas flows in the deposition chamber.

$\mathrm{CH}_{3} \mathrm{NH}_{3} \mathrm{PbI}_{3}$ films were made by a conversion of $\mathrm{PbI}_{2}$ films. First $\mathrm{PbI}_{2}$ films were deposited with ALD at $75{ }^{\circ} \mathrm{C}$ with the $\mathbf{0 P b}-\mathrm{SnI}_{4}$ process (Acros Organics, $>99 \%,-10$ mesh). ${ }^{34}$ Then a TLC plate $(0.75 \mathrm{~mL} / \mathrm{g}$ pore volume silica gel on Al foil, Sigma-Aldrich) was submerged in a $500 \mathrm{mg} / \mathrm{mL}$ solution of $\mathrm{CH}_{3} \mathrm{NH}_{3} \mathrm{I}(>99.0 \%$, TCI) in dimethylformamide $(\geq 99.9 \%$, Sigma-Aldrich) for $30 \mathrm{~min}$. The TLC plate was then dried in air by keeping it on a $60{ }^{\circ} \mathrm{C}$ hot plate for $30 \mathrm{~min}$. The dry plate was placed on the bottom of a steel cylinder $(\mathrm{V}=0.5 \mathrm{~L})$ with the MAI permeated $\mathrm{SiO}_{2}$ side facing up. An aluminum support holding the $\mathrm{PbI}_{2}$ film face down was then placed on the TLC plate. The design of the aluminum support is such that the distance between the MAI permeated $\mathrm{SiO}_{2}$ face of the TLC plate and the surface of the $\mathrm{PbI}_{2}$ film was ca. $1 \mathrm{~mm}$. After the loading the cylinder was cycled with $\mathrm{N}_{2}$ (AGA, 99.999\%)/vacuum three times and isolated with vacuum inside (ca. 1 mbar). The evacuated cylinder was heated in an air oven at $140{ }^{\circ} \mathrm{C}$ for $24 \mathrm{~h}$ to complete the conversion of $\mathrm{PbI}_{2}$ to $\mathrm{CH}_{3} \mathrm{NH}_{3} \mathrm{PbI}_{3}$. 


\subsubsection{Film Characterization}

Prior to characterization, the films were stored in closed containers filled with ambient air unless indicated otherwise. No significant oxidation is expected for PbS stored in air, ${ }^{225,233,234}$ but instead a thin (ca. $0.5 \mathrm{~nm}$ ) passivating $\mathrm{PbSO}_{3} / \mathrm{PbSO}_{4}$ layer can form upon prolonged exposure. ${ }^{225}$

Film thicknesses were measured with X-ray reflectivity (XRR, Rigaku SmartLab diffractometer). To measure XRR thickness maps, slits were used to limit the irradiated area ${ }^{235}$ to $\leq 5 \times 4 \mathrm{~mm}^{2}$ (at the angle range of interest), and an automated $\mathrm{XY}$ stage was used to measure a $7 \times 7$ matrix across the $5 \times 5 \mathrm{~cm}^{2}$ samples with $0.5 \mathrm{~cm}$ edge exclusion. Grazing incidence XRD (GIXRD) patterns were measured at $1^{\circ}$ incidence angle. Grain sizes were calculated by using the Scherrer equation and full width at half-maximum of the most intense reflection. Scherrer constants from Langford and Wilson et al. ${ }^{236}$ were used for the respective lattice planes assuming cubic crystallites. The instrumental broadening was defined by measuring $\mathrm{PbS}$ powder (99.9\%, Sigma-Aldrich) out-of-plane and in-plane with the same optics as used to measure PbS thin films.

The morphology of the films was studied with a Hitachi S-4800 field emission scanning electron microscope (FESEM) and with atomic force microscopy (AFM) by using a Veeco Multimode V instrument. AFM tapping mode images were captured in air using silicon probes with a nominal tip radius of $10 \mathrm{~nm}$ and a nominal spring constant of $3 \mathrm{~N} / \mathrm{m}$ (NFESP from Bruker). Images were flattened to remove artifacts caused by a sample tilt and a scanner bow. Resistivities were determined from sheet resistance measured with four-point probe (CPS Probe Station, Cascade Microtech combined with a Keithley 2400 SourceMeter) from films deposited on soda lime glass.

Hall effect measurements of films on sapphire substrates were made at room temperature with an Ecopia Van der Pauw HMS-5000 measurement system. Time-of-flight elastic

recoil detection analysis was performed using a $40 \mathrm{MeV}^{127} \mathrm{I}^{7+}$ beam. The incident angle of 
the beam was $16^{\circ}$, and the scattering angle was $40^{\circ}$. An FEI Quanta 3D 200i dual-beam microscope and standard lift-out procedures were used to prepare TEM cross-section specimens. An FEI Tecnai F20 microscope operated at $200 \mathrm{kV}$ was used for bright-field TEM imaging. An Oxford INCA 350 energy spectrometer connected to the FESEM was used for energy dispersive X-ray (EDS) measurements.

Transmittance of the films on sapphire substrates in the 190-3000 nm wavelength range was measured with an Agilent Cary 5000 spectrophotometer equipped with an internal diffuse reflectance measurement accessory (DRA-2500). The internal DRA consists of a $110 \mathrm{~mm}$ diameter integrating sphere with input and output apertures to perform reflectance and transmittance measurements. Sample of interest was mounted at the input aperture and the output aperture of the integrating sphere was covered with a standard reference material in the transmittance mode measurement. A Labsphere Spectralon SRM-99 diffuse reflectance standard was used for this purpose. Reference light was passed through the sample of interest when sample spectra were collected and the reference light intensity, in this case the emission light of Cary 5000, was measured without a sample. The scan speed was set to $10 \mathrm{~nm} / \mathrm{s}$ and step size to $1 \mathrm{~nm}$. Transmittance in the 3000$8000 \mathrm{~nm}$ wavelength range was measured with a Bruker Alpha II FTIR spectrometer. For Tauc plot construction direct allowed band gap was assumed for $\mathrm{PbS}$.

Water vapour transmission (WVTR) tests were performed, and results were calculated according to the ASTM standard E 96/E 96M-05 desiccant method with modifications in custom-built chambers that comply with the standard requirements. The relative humidity $(\mathrm{RH})$ gradient used in these measurements was $0 / 75 \%$ instead of the $0 / 50 \%$ in the standard. The exposed sample surface area was limited to $5 \mathrm{~cm}^{2}$ with an adhesive PTFE/aluminum masking. The temperature was $23{ }^{\circ} \mathrm{C}$. The temperature and $\mathrm{RH}$ of the cabinet were measured by using a Rotronic RH meter (Bassersdorf, Switzerland) before each weighing. The experiments were performed by using three replicates. The WVTR was calculated from the weight gain via equation WVTR $=(\Delta W / t) / A$, where $\Delta W$ is the 
weight gain, $t$ the time, and A the exposed surface area of the sample. The oxygen transmission rate and permeability were tested according to the ASTM standard D 3985-05 by using a Systech Illinois 8001 oxygen permeation analyzer (Johnsburg, IL). A mask (A Systech Illinois 8001 accessory) was used to limit the surface area of the samples to $5 \mathrm{~cm}^{2}$. The $\mathrm{O}_{2}$ partial pressure gradient was 1 atm with $\mathrm{N}_{2}$ on the receiving side, the temperature was set to $23{ }^{\circ} \mathrm{C}$, and the relative humidity of both gases $\left(\mathrm{N}_{2}\right.$ and $\left.\mathrm{O}_{2}\right)$ was $50 \%$. Two replicates of each sample were measured.

\subsubsection{Computational Details}

Density functional theory (DFT) was performed entirely with ORCA v4.2.1. ${ }^{237}$ Geometries were optimized using Head-Gordon's range-separated hybrid functional modified by Najibi and Goerigk ${ }^{238}$ to include Grimme’s dispersion correction with Becke-Johnson damping $^{239,240}[\omega \mathrm{B} 97 \mathrm{X}-\mathrm{D} 3(\mathrm{BJ})]$, Ahlrich's double- $\zeta$ split-valence quality basis set on all atoms (def2-SVP), ${ }^{241}$ and the Stuttgart ECP60MDF pseudopotential on $\mathrm{Pb} .{ }^{242}$ The resolution of the identity approximation for the Coulomb integrals with the chain of spheres approximation for the exchange term (RIJCOSX) ${ }^{243}$ with the def2/J auxiliary basis set ${ }^{244}$ was used to speed up the self-consistent field (SCF) step of all calculations. Frequency analysis was used to confirm there were no imaginary vibrational modes, and the geometries were minima on the potential energy surface. Numerical frequency calculations were performed to work around a known bug in the ORCA code involving analytical Hessian calculations together with the RIJCOSX approximation. Single-point calculations with a quadruple- $\zeta$ basis set (def2-QZVPP) were used with the thermochemical corrections from the previous frequency analysis. Enthalpy, entropy, and free energy changes of reaction were calculated by using Hess's law, with gas-to-solid transition enthalpy and entropy changes for $\mathrm{Pb}$ and $\mathrm{PbS}$ taken from the NIST Standard Reference Database Number 69. ${ }^{189}$ All structures, energies, orbitals, and wavefunctions are available upon request. 


\section{Chapter 4}

\section{Designing Volatility Into Thermally Stable Plumbylenes}

Modified from the original manuscript:

Goran Bačić, ${ }^{c}$ Jason D. Masuda, ${ }^{m}$ and Seán T. Barry. ${ }^{c}$ Designing Volatility Into Thermally Stable Plumbylenes, In Preparation, 2020.

c Department of Chemistry, Carleton University, 1125 Colonel By Drive, Ottawa, Ontario, K1S 5B6, Canada

$m$ Department of Chemistry, Saint Mary's University, 923 Robie Street, Halifax, Nova Scotia, B3H 3C3, Canada 


\section{Abstract}

Diamido plumbylenes have proven to be a successful framework for $\mathrm{Pb}$-containing atomic layer deposition (ALD) precursors due to their high volatility and reactivity, but their relatively low thermal stability restricts their use to low temperatures. Here, we explore the 1,3-diaza-2-plumba-4-silacyclobutane skeleton as a potential precursor framework. The champion compound, bis[lead(II) $N, N^{\prime}$-di-tert-butyl-1,1-dimethylsilanediamide] [(4Pb $\left.)_{2}\right]$, was volatile $\left(1\right.$ Torr at $112{ }^{\circ} \mathrm{C}$ ) and thermally stable at high temperatures (at least $300{ }^{\circ} \mathrm{C}$ ). Complex $(\mathbf{4 P b})_{2}$ was also a dimer, which we attempted to cleave by increased sterics, ancillary ligands, and pendant amino groups, but we found that the monomeric derivatives may be less volatile. Quantum chemical calculations revealed that dimerization of $(\mathbf{4 P b})_{2}$, while increasing the weight and surface area of the molecule, evens out the molecular electrostatic potential (MEP) of the molecule, likely leading to its good volatility. We performed a statistical analysis of the MEP of other synthesized and hypothetical plumbylenes in or-

der to begin developing a qualitative chemical basis for designing volatility in precursors, and finally speculate on effect of decreased sterics on volatility. 


\subsection{Introduction}

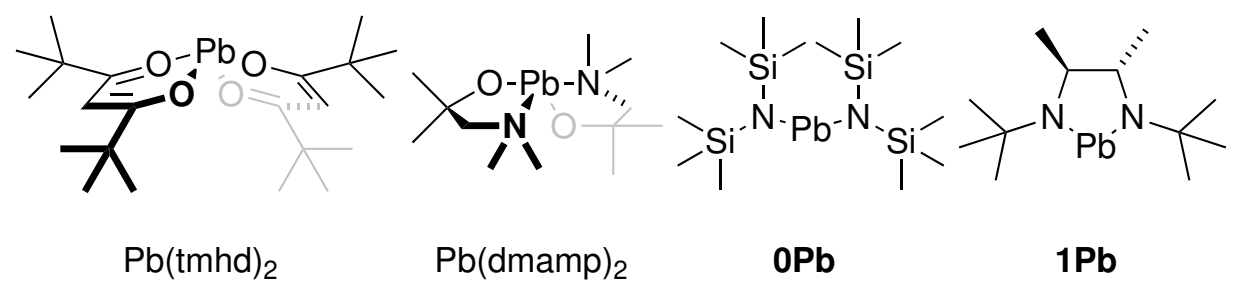

Figure 4.1: Known Pb-containing ALD precursors.

A rich diversity of $\mathrm{Pb}$-containing atomic layer deposition (ALD) precursors have been developed over the last 30 years, ${ }^{68}$ and two in particular have become the most popular: the older $\beta$-diketonate lead(II) bis(2,2,6,6-tetramethylheptane-3,5-dionate) $\left[\mathrm{Pb}(\mathrm{tmhd})_{2}\right],{ }^{35,161,245-249}$ and the newer amino-alkoxide lead(II) bis( $N, N$-dimethyl-3-amino-2-methyl-2-propoxide) $\left[\mathrm{Pb}(\mathrm{dmamp})_{2}\right] .{ }^{250-255}$ Both have been used for binary and complex oxides (i.e., with Ti, $\mathrm{Zr}$, and Hf $) ;{ }^{247,248,250-255}$ while $\mathrm{Pb}(\mathrm{tmhd})_{2}$ has been used to deposit all the chalcogenides, ${ }^{35,161,245,246,249}$ and can even achieve area-selective ALD on octadecyltrichlorosilane-patterned silicon substrates. ${ }^{246}$ However, neither are volatile enough to be used below $150{ }^{\circ} \mathrm{C}$, which precludes their use on thermally-sensitive substrates like hybrid organic-inorganic perovskites. In our group, we have focused on developing diamido plumbylenes because they are volatile, thermally stable, and chemically reactive species with easily modified frameworks. We found success with the acyclic lead(II) bis[bis(trimethylsilyl)amide] (0Pb) and the cyclic rac- $N, N^{\prime}$-di-tert-butyl-4,5-dimethyl-1,3-diaza-2-plumbacyclopenta-2-ylide $(\mathbf{1 P b})$ as precursors for ultralow-temperature atomic layer deposition (ALD) of $\mathrm{PbI}_{2}$ and $\mathrm{PbS}$ (Figure 4.1). ${ }^{34,256}$ Despite their effectiveness at low temperatures, both $\mathbf{0 P b}$ and $\mathbf{1 P b}$ are thermally unstable and undergo decomposition and chemical vapour deposition (CVD) above $155{ }^{\circ} \mathrm{C} .{ }^{34,256}$ Their useful temperature windows are narrowed further: the slightly lower volatility of $\mathbf{0 P b}$ restricts its use in ALD processes to only $65^{\circ} \mathrm{C}$ for $\mathrm{PbS}$ on methylammonium lead iodide (MAPI) perovskite thin films; ${ }^{256}$ and ALD of PbS with $\mathbf{1 P b}$ was restricted to $45-75{ }^{\circ} \mathrm{C}$ as an undesirable reduction to elemental lead(0) by $\mathrm{H}_{2} \mathrm{~S}$ becomes 
competitive above $95{ }^{\circ} \mathrm{C} .{ }^{256}$ To the best of our knowledge, a Pb-containing precursor with high thermal stability, volatility, and reactivity has yet to be reported.

Here, we explore 1,3-diaza-2-plumba-4-silacyclobutanes experimentally and theoretically as a new class of potential ALD precursors. The champion compound bis[lead(II) $N, N^{\prime}$-di-tert-butyl-1,1-dimethylsilanediamide] $\left[(\mathbf{4 P b})_{2}\right]$ has high thermal stability, good volatility, and a straightforward low-cost high-yield synthesis, making it a prime candidate for a low- and high-temperature ALD precursor. While $(\mathbf{4 P b})_{2}$ was more volatile than $\mathbf{0 P b}$, it was still significantly less volatile than $\mathbf{1 P b}$, so we set out to modify this framework and produce more volatile derivatives. Surprisingly, modifications that have shown success in traditional precursor design, like using bulkier and less-symmetric alkyl groups to lower the melting point, ${ }^{257-259}$ actually decreased the volatility of derivatives. Below, we investigate how to cleave the $(\mathbf{4 P b})_{2}$ dimer through ligand design. We show that the dimer can be disrupted with pendant amines or ancillary ligands, but at a cost to volatility or stability. Our counter-intuitive results were rationalized by computing the molecular electrostatic potential (MEP) surfaces of volatile $\mathrm{Pb}$-containing precursors toward a general interactions properties function (GIPF) for vapour pressures of precursors in general. Finally, we use our theoretical results to rationally design hypothetical plumbylenes that may also be volatile and stable.

\subsection{Results and Discussion}

\subsubsection{Lead(II) $N, N^{\prime}$-di-tert-butyl-1,1-dimethylsilanediamide}

Four-membered 1,3-diaza-2-plumba-4-silacyclobutanes first appeared in the literature when lead(II) N,N'-di-tert-butyl-1,1-dimethylsilanediamide $\mathbf{4 P b}$ was prepared and characterized by Veith in $1982 .{ }^{260}$ Since then, $\mathbf{4 P b}$ has been used as a precursor to group 14 heterocubanes like $\left[\mathrm{Pb}\left(\mathrm{N}^{t} \mathrm{Bu}\right)\right]_{4}$ by transamination with tert-butylamine, ${ }^{260}$ plumbocene by $\mathrm{C}-\mathrm{H}$ activation of free cyclopentadiene, ${ }^{261}$ tris[lead(II) bis(tert-butoxide)] by reaction with 


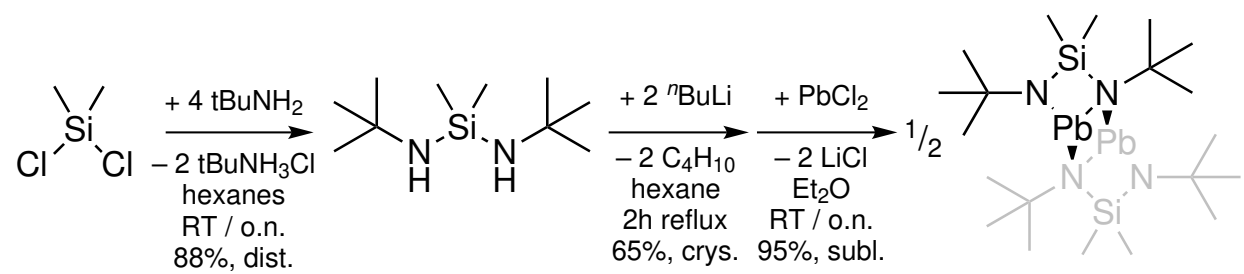

$(4 \mathrm{~Pb})_{2}$

Figure 4.2: Three-step synthesis of $(\mathbf{4 P b})_{2}$ from basic starting materials.

tert-butanol, ${ }^{262}$ and the heterobimetallic plumbate $\left[\mathrm{Ti}\left(\mathrm{O}^{i} \mathrm{Pr}\right)_{3}\right]\left[\mathrm{OPb}_{4}\left(\mathrm{~N}^{t} \mathrm{Bu}\right)_{3}\right]$ by concomitant hydrolysis and reaction with $\mathrm{Ti}\left(\mathrm{O}^{i} \mathrm{Pr}\right)_{4}{ }^{263}$ It has also been used in metathesis reactions with phosphorus(III) ${ }^{264,265}$ and tin(IV) chlorides. ${ }^{266}$ The sole example of $\mathbf{4 P b}$ in materials chemistry was as a solution-phase reduction with aluminum hydrides to metallic $\mathrm{Pb}(0)$ nanoparticles and foams. ${ }^{267}$ Three other 1,3-diaza-2-plumba-4-silacyclobutanes have previously been isolated and structurally characterized: bis[lead(II) $N, N^{\prime}$-di-tert-butyl- $N$ -

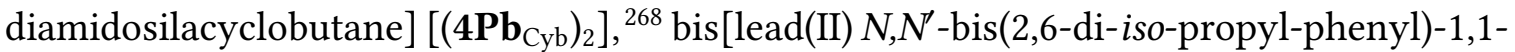

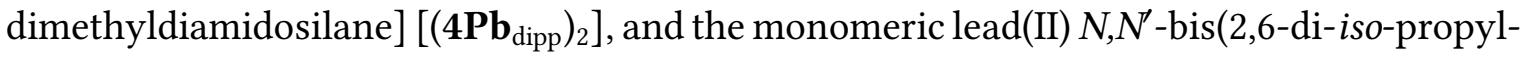
phenyl)-1,1-diphenyldiamidosilane $\left[\mathbf{4} \mathbf{P b}_{\mathrm{Ph}} 4 .{ }^{269}\right.$
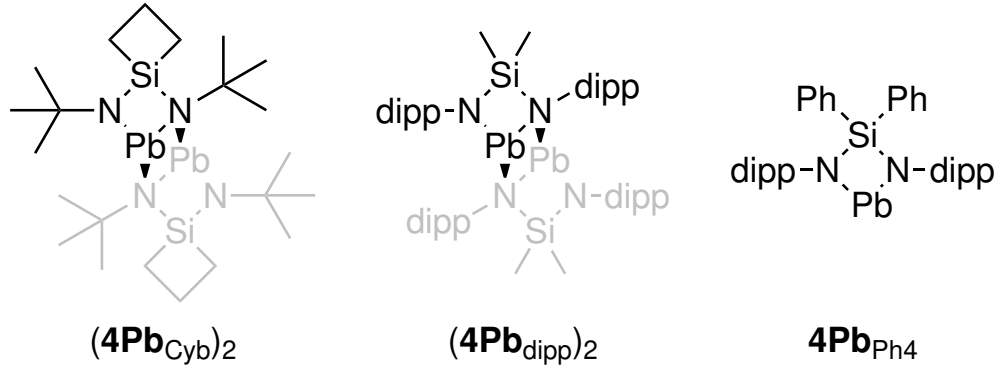

Figure 4.3: Structurally characterized 1,3-diaza-2-plumba-4-silacyclobutanes. ( $\mathrm{Ph}=$ phenyl, dipp $=$ di-iso-propylphenyl.)

We were encouraged to evaluate $\mathbf{4 P b}$ as a potential precursor for several reasons, despite previous efforts undertaken by our group to avoid dimerization in precursor molecules containing heavy metals. ${ }^{81,85,182}$ First, NMR spectroscopy suggested $\mathbf{4 P b}$ was monomeric in solution, ${ }^{260,270,271}$ and electron-impact mass spectrometry suggested it was monomeric in the gas phase. ${ }^{260}$ Pure $4 \mathbf{P b}$ was reported to melt at $85{ }^{\circ} \mathrm{C} .{ }^{260}$ The sharp reversible melt- 
ing points of the di-iso-propylphenyl-derivatives $\left(\mathbf{4} \mathbf{P b}_{\mathrm{dipp}}\right)_{2}$ and $\mathbf{4} \mathbf{P b}_{\mathrm{Ph} 4}\left(148\right.$ and $215{ }^{\circ} \mathrm{C}$, respectively) suggested $\mathbf{4 P b}$ and its derivatives had high thermal stability. Both the lighter germanium(II) and tin(II) analogues were distillable liquids (b.p. $=40$ and $56^{\circ} \mathrm{C}$ at $100 \mathrm{mTorr}$, respectively), ${ }^{260}$ but no attempted distillation of $\mathbf{4 P b}$ was reported. And, finally, $\mathbf{4 P b}$ was sufficiently volatile to be vaporized between $25-80{ }^{\circ} \mathrm{C}$ under vacuum. ${ }^{272}$

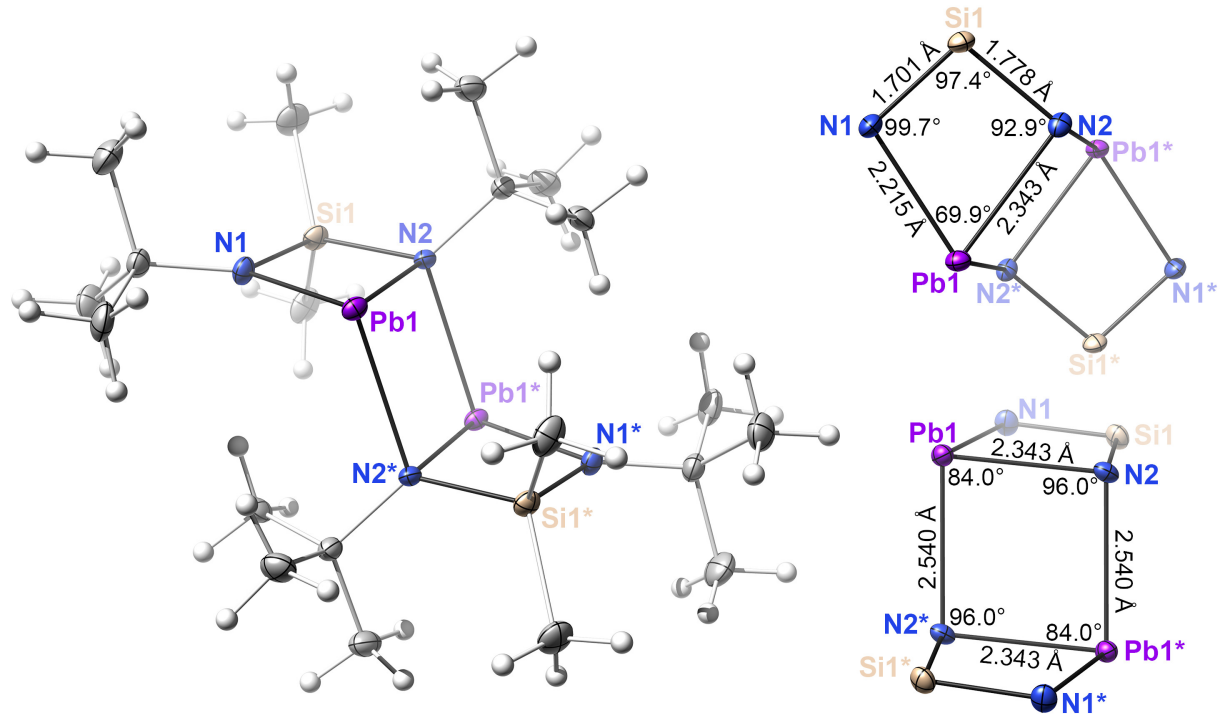

Figure 4.4: Structure of $(\mathbf{4 P b})_{2}$ from single-crystal X-ray diffraction (left), and geometric quantities of the intramolecular (top-right) and intermolecular (bottom-right) heterocycles with carbon and hydrogen omitted for clarity. Only one enantiomer present in the crystal is shown. Ellipsoids are drawn at $50 \%$ and hydrogens are drawn as spheres.

The complex $\mathbf{4 P b}$ was easily prepared by salt-metathesis between the dilithiated diamidosilane ligand and $\mathrm{PbCl}_{2} \cdot{ }^{260}$ High yields (95\%) of a light-yellow thermochromic solid were obtained after vacuum sublimation $\left(100{ }^{\circ} \mathrm{C} / 40 \mathrm{mTorr}\right)$, and we found no difficulty or decrease in yield when the synthesis was scaled-up to $15 \mathrm{~g}$. Neither solutions nor crystals of $\mathbf{4 P b}$ were sensitive to light, unlike $\mathbf{0 P b}$ and $\mathbf{1 P b}$ which darken under illumination. Large hexagonal block single-crystals suitable for X-ray diffraction were grown by cooling a toluene solution of $\mathbf{4 P b}$ overnight at $-49{ }^{\circ} \mathrm{C}$. The complex was found to be solely dimeric $(\mathbf{4 P b})_{2}$ in the solid-state (Figure 4.4$)$. Its structure resembled that of $\left(\mathbf{4 P} \mathbf{b}_{\mathrm{Cyb}}\right)_{2}$ except with elongated intermolecular $\mathrm{Pb}-\mathrm{N}^{*}$ distances $(2.540(3) \AA$ vs. 2.47(1) $\AA)$, 
a slightly larger $\mathrm{N}-\mathrm{Pb}-\mathrm{N}$ angle $\left(69.95(10)^{\circ}\right.$ vs. $\left.69.0(4)^{\circ}\right)$, and more square $\mathrm{N}-\mathrm{Pb}-\mathrm{N}^{*}$ and $\mathrm{Pb}-\mathrm{N}-\mathrm{Pb}^{*}$ angles $\left(83.99(10)^{\circ}\right.$ and $96.00(10)^{\circ}$ vs. 80.6(4) and $\left.99.4(4)^{\circ}\right){ }^{\circ}{ }^{268}$ The reduced steric bulk of the cyclobutyl-backbone likely allowed better overlap of the $2 \mathrm{sp}^{3}$-hybridized lonepair of the intermolecularly coordinating nitrogen and the empty electrophilic $6 \mathrm{p}_{z}^{0}$ orbital on $\mathrm{Pb}(\mathrm{II})$, resulting in shorter interatomic distances. There was also a close resemblance to $\left(\mathbf{4} \mathbf{P} \mathbf{b}_{\text {dipp }}\right)_{2}$, except $(\mathbf{4 P b})_{2}$ had shorter intermolecular $\mathrm{Pb}-\mathrm{N}^{\star}$ distances, likely due to the slightly smaller steric bulk of the tert-butyl group. ${ }^{269}$
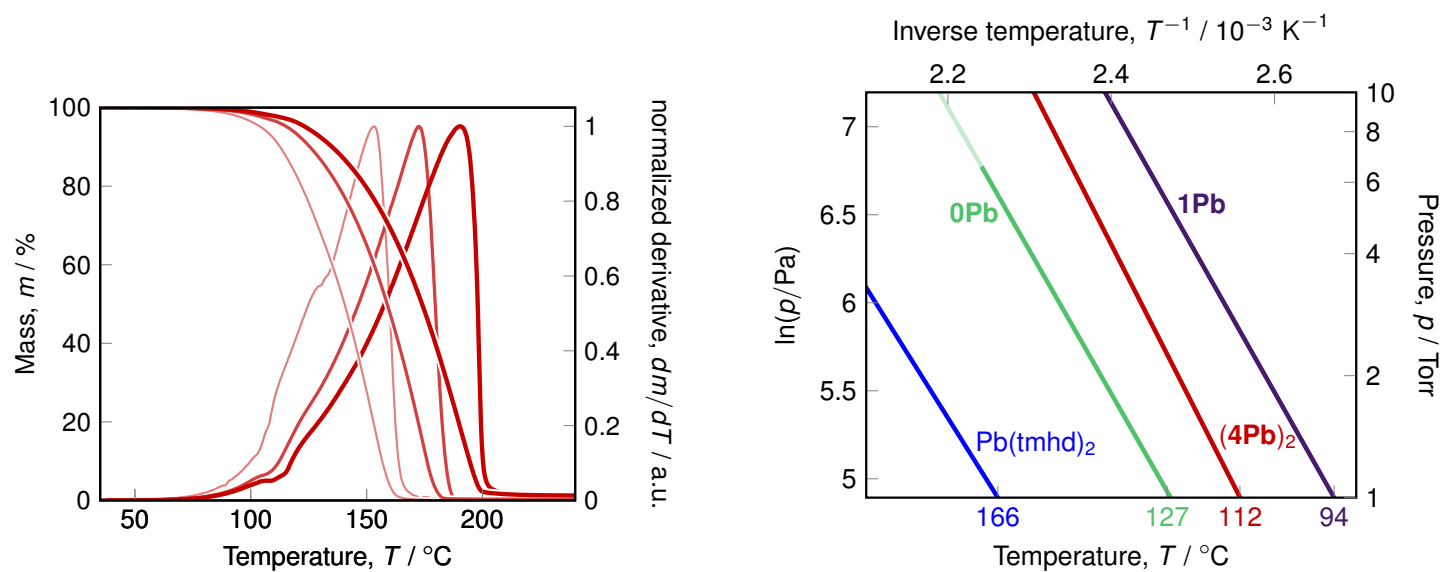

Figure 4.5: Non-isothermal thermogravimetric analysis (TGA) of $(\mathbf{4 P b})_{2}$ with increasing initial mass loading (ca. 10, 20, and $40 \mathrm{mg}$ ) with normalized first derivatives (left), and Clausius-Clapeyron vapour pressure-temperature relationships (right) for $(\mathbf{4 P b})_{2}$ and the known precursors shown in Figure 4.1.

Thermogravimetric analyis (TGA) of $(\mathbf{4 P b})_{2}$ showed volatility between $\mathbf{0 P b}$ and $\mathbf{1 P b}$ ( 1 Torr vapour pressure at $112{ }^{\circ} \mathrm{C}$ ) and excellent thermal stability (Figure 4.5). Thermalstress tests, either by increasing the initial mass loading or the heating rate, resulted in no increase in residual mass and no visible residue was ever left behind, suggesting no decomposition occurred. It evaporated completely even after experiencing temperatures above $250{ }^{\circ} \mathrm{C}$ with heating rates up to $30{ }^{\circ} \mathrm{C} \mathrm{min}^{-1}$. In our home-built ALD reactor, we observed no decomposition on HF-dipped silicon, sodalime glass, nor gold substrates when $3 \mathrm{~g}(\mathbf{4 P b})_{2}$, held at $100{ }^{\circ} \mathrm{C}$, was flowed into the chamber heated to $300{ }^{\circ} \mathrm{C}$ over about $3 \mathrm{~h}$. The complex $(\mathbf{4 P b})_{2}$ shows great potential as a precursor for low- and high-temperature deposition. Since $(\mathbf{4 P b})_{2}$ was a dimer in the solid state, we presumed it dissociated into 
monomers before evaporating, and took the unusual shape of the TGA curves to be a result of this reaction.

High-level quantum chemical calculations (DLPNO-CCSD(T), see Experimental for details) gave the gas-phase dissociation energy $\Delta G_{\mathrm{D}}^{\circ}$ of $(\mathbf{4 P b})_{2}$ to be $13.4 \mathrm{kcal} / \mathrm{mol}$, comparable to that of the trimethylaluminum dimer $\left(\mathrm{Me}_{3} \mathrm{Al}\right)_{2},{ }^{273,274}$ so the concentration of monomeric $\mathbf{4 P b}$ during evaporation was likely never higher than $1 \%{ }^{275}$ The strong intermolecular interaction of the dimer could be clearly seen when non-covalent interactions (NCIs) were visualized as red donut rings around the $\mathrm{Pb}-\mathrm{N}^{*}$ bonds (Figure 4.6). ${ }^{276}$ During TGA of complex $(\mathbf{4 P b})_{2}$, melting was clearly observed as a dip in the derivative curve after mass-loss (i.e., sublimation) began. Once the sample had completely melted, it began evaporating under zeroth-order kinetics again. However, for complex $(\mathbf{4 P b})_{2}$, the derivative curve then broadens and skews to the right, which typically indicates the onset of another reaction (i.e., decomposition to elemental lead). ${ }^{182}$

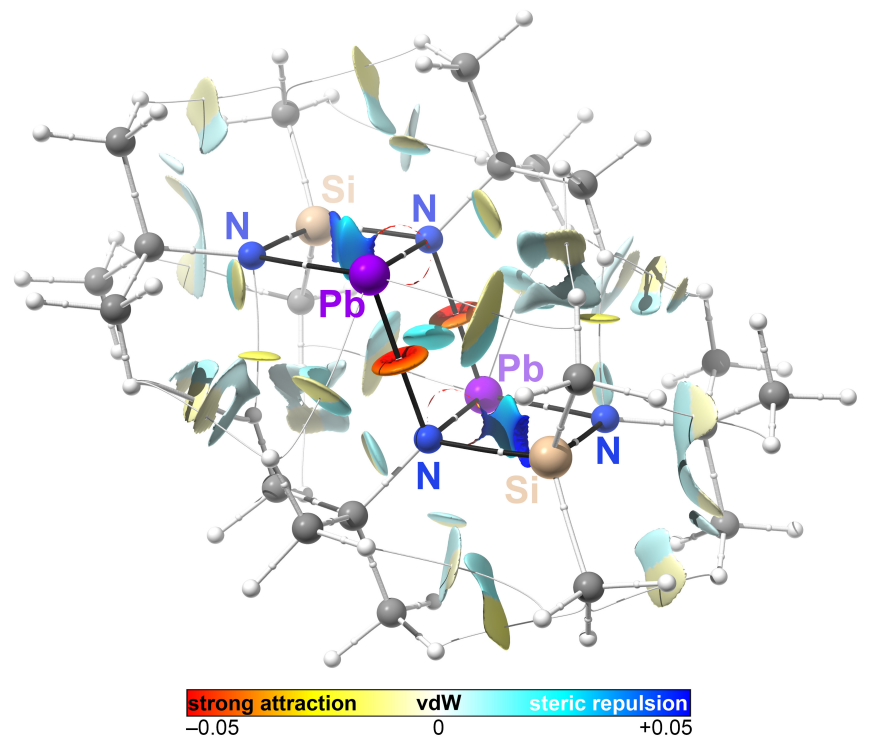

Figure 4.6: Atoms-in-molecules (QTAIM) graphs and non-covalent interaction (NCI) analysis of $(\mathbf{4 P b}) 2$ calculated with DFT [ZORA-def2-QZVPP(SARC-ZORA-TZVPP)//revPBE/ZORA-def2SVP(SARC-ZORA-TZVP)] in the gas-phase. Atom interaction lines (AILs) connecting $(+3,-3)$ critical points (i.e., nuclei) through $(+3,-1)$ bond critical points (white spheres along AILs) are drawn black if terminating at $\mathrm{Pb}$ and white otherwise. Ring and cage critical points are omitted for clarity. Reduced density gradient (RDG) surface drawn at a contour value of $0.333 \mathrm{a} \mathrm{u}$ and their colour is mapped to the value of $\operatorname{sign}\left(\lambda_{2}\right) \rho$. 
The shape of the first-derivative of the TGA curves can be used to determine how many and which type of reactions occurred during an experiment. ${ }^{106,107}$ Since evaporation is a zeroth-order kinetic process, the derivative of mass-loss with respect to time $\mathrm{d} m / \mathrm{d} t$ under non-isothermal conditions will be a negative exponential if the material is only undergoing evaporation. ${ }^{105}$ Overlapping reactions such as melting or decomposition will cause deviation from this ideal shape beginning at their onset, making the shape of the derivative curve helpful for qualifying the thermal stability of precursor molecules. ${ }^{108}$ We were unable to deconvolute any overlapping reactions by TGA with different heating rates and began to suspect that the sample surface area was changing during evaporation. Samples of $(\mathbf{4 P b})_{2}$ were heated to above their melting point $\left(100{ }^{\circ} \mathrm{C}\right)$ for ten minutes, then cooled to resolidify into a puck with a constant area. We found that molten $(\mathbf{4 P b})_{2}$ beaded up rather than wetting the Pt pans, leaving behind large isolated crystals when cooled. This would explain the changing rate of mass-loss during the non-isothermal experiments: as the sample evaporates, the liquid will bead up, the evaporating surface area will change, and so will the rate of evaporation. We found no indication of overlapping reactions when we used sufficient initial sample masses to fill the pan with liquid.

\subsubsection{Increasing Steric Bulk}

Motivated by our success with $(\mathbf{4 P b})_{2}$, we wanted to try and improve the volatility by preparing modified derivatives according to traditional precursor design strategies. First, we tried introducing bulkier and less-symmetrical functional

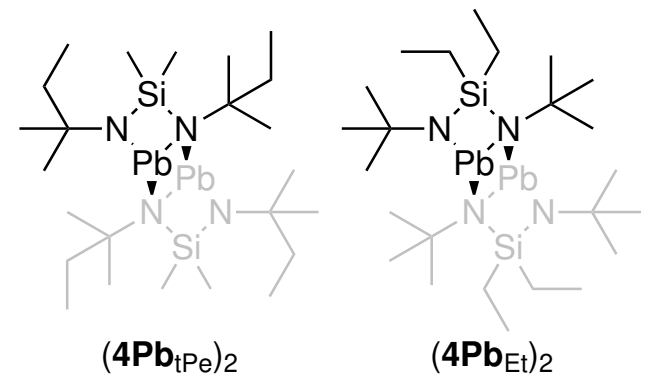
groups to lower the melting point by replacing tert-butyl with tert-pentyl, analagous to iso-propyl being replaced by \pm -sec-butyl in acetamidinates, ${ }^{257-259}$ A tertiary alkyl group on the amido moiety was necessary to sta- 
bilize the plumbylene from $\beta$-hydrogen elimination, so the commercially available tertpentylamine was used instead of other secondary or primary amines.

The tert-pentyl derivative bis[lead(II) $N, N^{\prime}$-di-tert-pentyl-1,1-dimethyl-1,1-diamidosilane] $\left(\mathbf{4} \mathbf{P} \mathbf{b}_{\mathrm{tPe}}\right)_{2}$ was prepared in a similar manner to $(\mathbf{4 P b})_{2}$. It was also a dimer in the solid state with a structure similar to its tert-butyl parent, except the intermolecular $\mathrm{Pb}-\mathrm{N}^{*}$ distance was slightly shorter (2.5253(12) $\AA$ vs $2.540(3) \AA)$. This was surprising in light of recent reports of significantly greater steric hindrance of tert-pentyl in comparison to tert-butyl for amines and other $\mathrm{N}$-containing organic compounds. ${ }^{27,278}$ The modification did lower the melting point $\left(65^{\circ} \mathrm{C}\right)$, but worsened the volatility $\left(1\right.$ Torr vapour pressure at $\left.116{ }^{\circ} \mathrm{C}\right)$. All things considered - lower cost, better availability, higher yield, better thermal properties - the tert-butyl parent $(\mathbf{4 P b})_{2}$ was a significantly better precursor candidate over the tert-pentyl derivative despite its higher melting point.

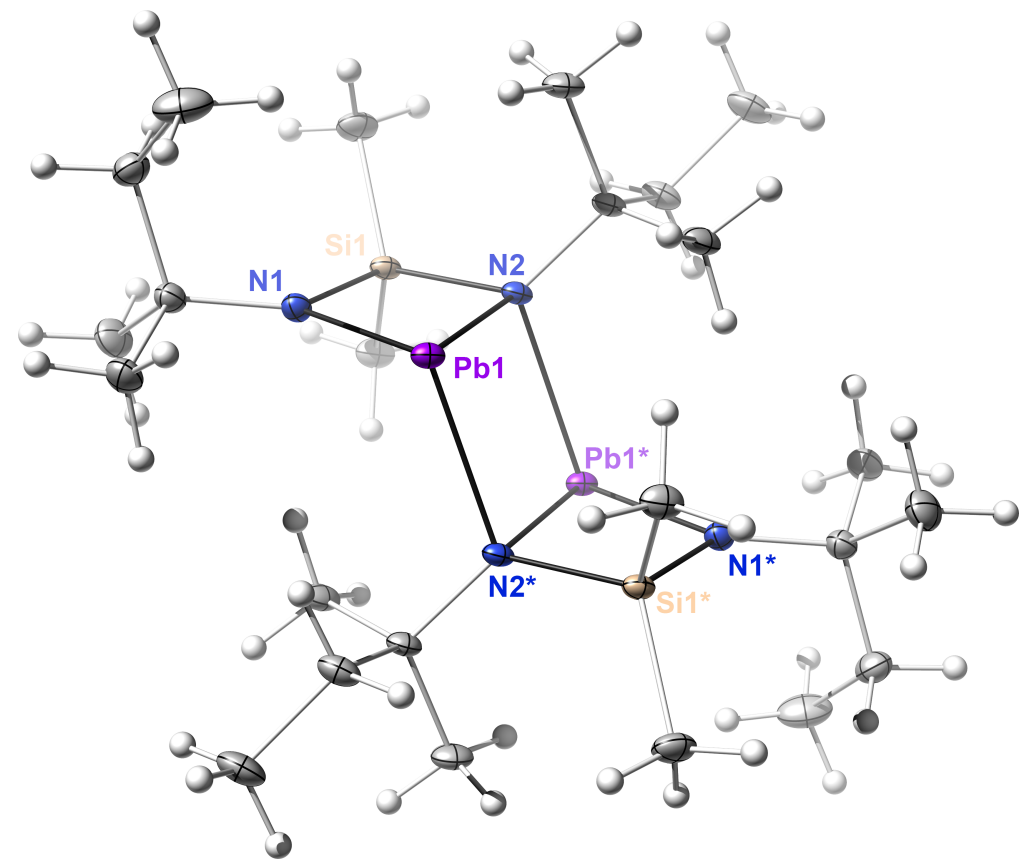

Figure 4.7: Structure of $\left(\mathbf{4} \mathrm{Pb}_{\mathrm{tPe}}\right)_{2}$ from single-crystal X-ray diffraction. Only one enantiomer present in the crystal is shown. Ellipsoids are drawn at $50 \%$ and hydrogens are drawn as spheres.

The alkyl groups on silicon can also be substituted easily by starting with the appropriate dialkyldichlorosilane. Most are not as inexpensive and readily-available as dimethyldichlorosilane, so we limited this study to theoretically evaluating the diethylsilyl-derivative 


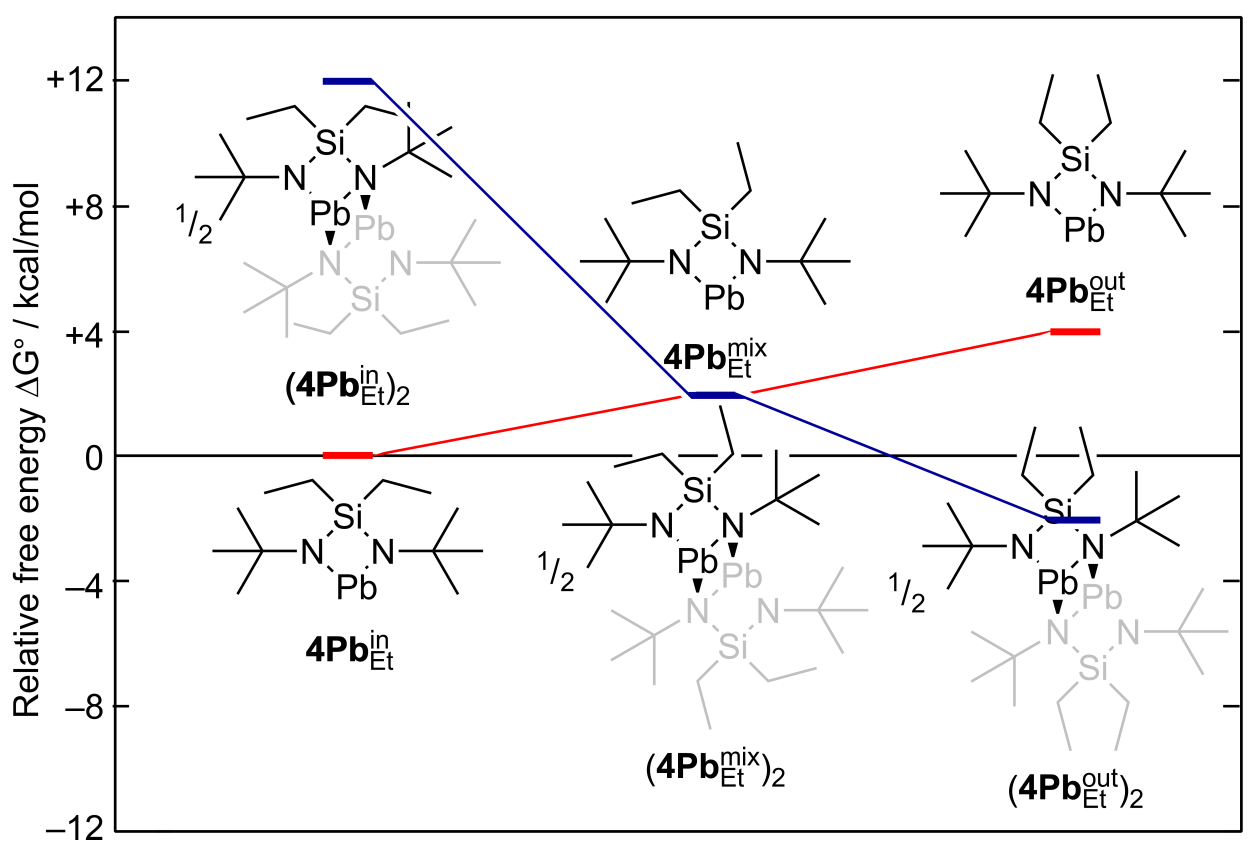

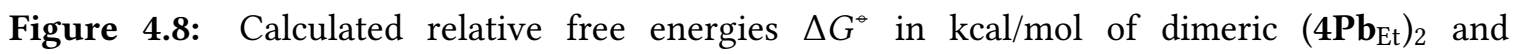
monomeric $4 \mathbf{P b}_{\mathrm{Et}}$ conformers in the gas phase [DLPNO-CCSD(T)/ZORA-def2-QZVPP(SARCZORA-TZVPP)//revPBE/ZORA-def2-SVP(SARC-ZORA-TZVP)].

bis[lead(II) $N, N^{\prime}$-di-tert-butyl-1,1-diamido-1,1-diethylsilane] $\left[\left(\mathbf{4} \mathbf{P b} \mathbf{b}_{\mathrm{Et}}\right)_{2}\right]$. Since there are many different possible conformers, we narrowed our calculations to three dimer conformations and their respective monomers: both ethyl groups endo $\left[\left(\mathbf{4} \mathbf{P} \mathbf{b}_{\mathrm{Et}}^{\mathrm{in}}\right)_{2}\right]$, both ethyl groups exo $\left[\left(\mathbf{4} \mathbf{P b} \mathbf{b}_{\mathrm{Et}}^{\text {out }}\right)_{2}\right]$, and the "top" ethyl groups endo and the bottom exo $\left[\left(\mathbf{4} \mathbf{P b} \mathbf{b}_{\mathrm{Et}}^{\text {mix }}\right)_{2}\right]$ (Fig-

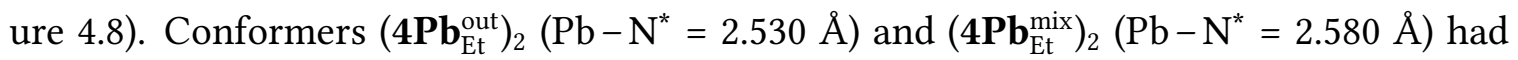
geometries very similar to $(\mathbf{4 P b})_{2}$ since the orientation of the ethyl groups away from the tert-butyl groups on the ring gave them an effective steric bulk similar to methyl. On the other hand, the dimer $\left(\mathbf{4} \mathbf{P} \mathbf{b}_{\mathrm{Et}}^{\mathrm{in}}\right)_{2}$ was destabilized toward the monomer and the increased steric bulk of the ethyl groups caused significant elongation of the $\mathrm{Pb}-\mathrm{N}^{*}$ intermolecular distances $\left(\mathrm{Pb}-\mathrm{N}^{*}=2.780 \AA\right)$. It was surprising that the monomer $\mathbf{4} \mathbf{P b}$ 解 was more stable than the other monomers $\mathbf{4} \mathbf{P b}_{\mathrm{Et}}^{\text {mix }}$ and $\mathbf{4} \mathbf{P b}_{\mathrm{Et}}^{\text {out }}\left(\Delta G^{\star}=-1.8\right.$ and $\left.-3.9 \mathrm{kcal} / \mathrm{mol}\right)$, and only slightly less favourable than the most stable dimer conformation $\left(\mathbf{4} \mathbf{P} \mathbf{b}_{\mathrm{Et}}^{\text {out }}\right)_{2}\left(\Delta G^{\circ}=\right.$ $+2.0 \mathrm{kcal} / \mathrm{mol})$. The relative stabilities of the monomers was likely due to a stabilizing dispersion interaction between the ethyl and tert-butyl groups on the ligand, but there was 
no obvious indication of that according to an NCI analysis. While our calculations suggest the ethyl-derivative $\left(\mathbf{4} \mathbf{P b} \mathbf{b}_{\mathrm{Et}}\right)_{2}$ would dissociate at much lower temperatures than its parent $(\mathbf{4 P b})_{2}$, it remains an open question whether this will improve volatility, especially in light of the findings discussed below.

\subsubsection{Ancillary Ligands}

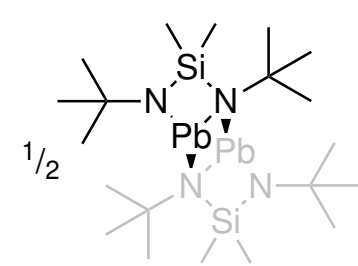

$(4 \mathrm{~Pb})_{2}$

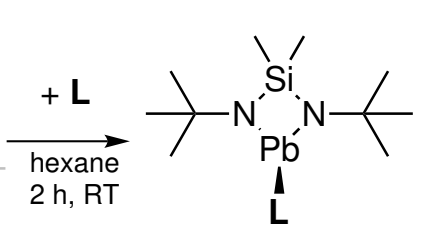

$(4 \mathrm{~Pb})(\mathrm{L})$

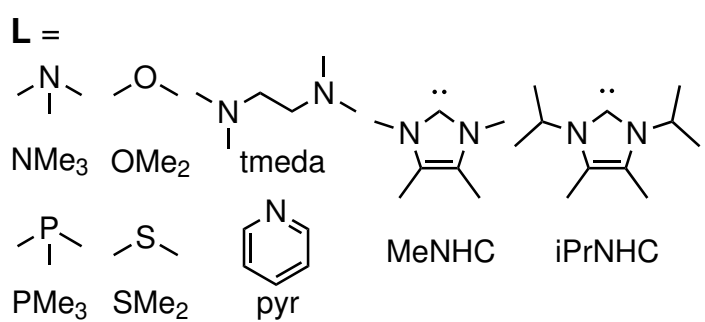

$\mathrm{PMe}_{3} \mathrm{SMe}_{2} \quad$ pyr

Figure 4.9: Proposed adduct formation of $(\mathbf{4 P b})_{2}$ with small readily-available ligands.

Ancillary ligands have been used in vapour-phase precursor design because they raise the possibility of fine-tuning or improving the physicochemical properties of known precursors. ${ }^{81,85,279}$ Plumbylenes are electrophilic due to the empty $6 \mathrm{p}_{z}^{0}$ orbital on $\mathrm{Pb}(\mathrm{II})$, so a suitable Lewis base could possibly cleave the dimer of $(\mathbf{4 P b})_{2}$ by forming an adduct with the monomer. There are many readily-available Lewis bases, so we decided to evaluate simple, small, and volatile candidates theoretically first (Figure 4.9). We evaluated the following representative members of the various types: dimethylether $\left(\mathrm{Me}_{2} \mathrm{O}\right)$, dimethylsulfide $\left(\mathrm{Me}_{2} \mathrm{~S}\right)$, trimethylamine $\left(\mathrm{NMe}_{3}\right)$, trimethylphosphine $\left(\mathrm{PMe}_{3}\right)$, pyridine (pyr), $N, N, N^{\prime}, N^{\prime}-$ tetramethylethane-1,2-diamine (tmeda), and the $N$-heterocyclic carbenes (NHCs) 1,3,4,5tetramethylimidazol-2-ylidene (MeNHC) and $N^{1}, N^{3}$-di-iso-propyl-4,5-dimethylimidazol2-ylidene (iPrNHC). Every ligand bonded exothermically with the monomer in the gas phase (Table 4.1). The majority of the adducts were only weakly favourable $\left(\Delta G^{\star} \geq\right.$ $-5 \mathrm{kcal} / \mathrm{mol}$ ), but the NHCs were predicted to form stable adducts compared to the free monomer in the gas-phase $\left(\Delta G^{\star}\right.$ ca. $\left.-15 \mathrm{kcal} / \mathrm{mol}\right)$. Compared to the dimer $(4 \mathbf{P b})_{2}$, only the NHCs bound favourably, and the other ligands did not even show significant exothermic- 
Table 4.1: Standard free energies and enthalpies of dimerization and adduct formation $(\mathbf{4 P b})_{2} \cdot{ }^{\mathrm{a}}$

\begin{tabular}{|c|c|c|}
\hline Reaction & $\begin{array}{c}\Delta G^{\ominus} \\
\mathrm{kcal} / \mathrm{mol}\end{array}$ & $\begin{array}{c}\Delta H^{\ominus} \\
\mathrm{kcal} / \mathrm{mol}\end{array}$ \\
\hline$\frac{1}{2}(\mathbf{4 P b})_{2} \longrightarrow \mathbf{4 P b}$ & 6.70 & 15.5 \\
\hline $4 \mathrm{~Pb}+\mathrm{NMe}_{3} \longrightarrow(4 \mathrm{~Pb})\left(\mathrm{NMe}_{3}\right)$ & -4.30 & -17.35 \\
\hline $4 \mathrm{~Pb}+\mathrm{PMe}_{3} \longrightarrow(4 \mathrm{~Pb})\left(\mathrm{PMe}_{3}\right)$ & -0.79 & -13.26 \\
\hline $4 \mathbf{P b}+\mathrm{OMe}_{2} \longrightarrow(\mathbf{4 P b})\left(\mathrm{OMe}_{2}\right)$ & 0.72 & -11.17 \\
\hline $4 \mathbf{P b}+\mathrm{SMe}_{2} \longrightarrow(\mathbf{4 P b})\left(\mathrm{SMe}_{2}\right)$ & 1.23 & -11.38 \\
\hline $4 \mathbf{P b}+\mathrm{pyr} \longrightarrow(\mathbf{4 P b})(\mathrm{pyr})$ & -3.68 & -16.35 \\
\hline $4 \mathrm{~Pb}+$ tmeda $\longrightarrow(4 \mathrm{~Pb})($ tmeda $)$ & -5.35 & -20.36 \\
\hline $4 \mathrm{~Pb}+\mathrm{MeNHC} \longrightarrow(4 \mathrm{~Pb})(\mathrm{MeNHC})$ & -15.20 & -28.60 \\
\hline $\mathbf{4 P b}+\mathrm{iPrNHC} \longrightarrow(\mathbf{4 P b})(\mathrm{iPrNHC})$ & -14.43 & -29.36 \\
\hline$\frac{1}{2}(\mathbf{4 P b})_{2}+\mathrm{NMe}_{3} \longrightarrow(\mathbf{4 P b})\left(\mathrm{NMe}_{3}\right)$ & 2.39 & -1.88 \\
\hline$\frac{1}{2}(\mathbf{4 P b})_{2}+\mathrm{PMe}_{3} \longrightarrow(\mathbf{4 P b})\left(\mathrm{PMe}_{3}\right)$ & 5.90 & 2.21 \\
\hline$\frac{1}{2}(\mathbf{4 P b})_{2}+\mathrm{OMe}_{2} \longrightarrow(\mathbf{4 P b})\left(\mathrm{OMe}_{2}\right)$ & 7.41 & 4.30 \\
\hline$\frac{1}{2}(\mathbf{4 P b})_{2}+\mathrm{SMe}_{2} \longrightarrow(\mathbf{4 P b})\left(\mathrm{SMe}_{2}\right)$ & 7.92 & 4.08 \\
\hline$\frac{1}{2}(\mathbf{4 P b})_{2}+\mathrm{pyr} \longrightarrow(\mathbf{4 P b})(\mathrm{pyr})$ & 3.01 & -0.89 \\
\hline$\frac{1}{2}(\mathbf{4 P b})_{2}+$ tmeda $\longrightarrow(\mathbf{4 P b})($ tmeda $)$ & 1.34 & -4.90 \\
\hline$\frac{1}{2}(\mathbf{4 P b})_{2}+\mathrm{MeNHC} \longrightarrow(\mathbf{4 P b})(\mathrm{MeNHC})$ & -8.50 & -13.14 \\
\hline$\frac{1}{2}(\mathbf{4 P b})_{2}+\mathrm{iPrNHC} \longrightarrow(\mathbf{4 P b})(\mathrm{iPrNHC})$ & -7.74 & -13.90 \\
\hline
\end{tabular}

Table 4.2: Mayer bond order (MBO), Wiberg bond index (WBI), and QTAIM topological parameters of $\mathbf{4 P b}$ and its adducts. ${ }^{\mathrm{a}}$

\begin{tabular}{clccccl}
\hline Adduct & Bond & MBO & WBI & $\rho(\mathbf{r})$ & $-\nabla^{2} \rho(\mathbf{r})$ & $\epsilon$ \\
\hline \multirow{2}{*}{$\mathbf{P b})_{2}$} & $\mathrm{~Pb}-\mathrm{N}_{\text {endo }}$ & 0.317 & 0.719 & 0.0507 & 0.101 & 0.0755 \\
& $\mathrm{~Pb}-\mathrm{N}_{\text {exo }}$ & 0.644 & 0.957 & 0.0864 & 0.230 & 0.138 \\
$\mathrm{NMe}_{3}$ & $\mathrm{~Pb}-\mathrm{N}$ & 0.307 & 0.620 & 0.0670 & 0.150 & 0.0545 \\
$\mathrm{OMe}_{2}$ & $\mathrm{~Pb}-\mathrm{O}$ & 0.178 & 0.403 & 0.0341 & 0.0650 & 0.0233 \\
$\mathrm{SMe}_{2}$ & $\mathrm{~Pb}-\mathrm{S}$ & 0.241 & 0.612 & 0.0262 & 0.0355 & 0.0231 \\
$\mathrm{pyr}$ & $\mathrm{Pb}-\mathrm{N}$ & 0.227 & 0.467 & 0.0344 & 0.0782 & 0.0575 \\
tmeda & $\mathrm{Pb}-\mathrm{N}$ & 0.274 & 0.351 & 0.0288 & 0.0534 & 0.0221 \\
MeNHC & $\mathrm{Pb}-\mathrm{N}^{\prime}$ & 0.067 & 0.154 & 0.0102 & 0.0297 & 0.323 \\
iPrNHC & $\mathrm{Pb}-\mathrm{C}$ & 0.381 & 0.790 & 0.0522 & 0.0891 & 0.0553 \\
\hline
\end{tabular}

a ZORA-def2-QZVPP(SARC-ZORA-TZVPP)//revPBE/ZORA-def2-SVP(SARCZORA-TZVP). 
ity, suggesting their $\mathbf{4 P b} \leftarrow \mathbf{L}$ bonds would be too weak to cleave the dimer. The differences in ligand bonding to the $\mathbf{4 P b}$ was visualized by NCI analysis, with relatively weak NCIs being seen for all ligands but the NHCs (Figure 4.10). It appeared that most of the ligands would not cleave the dimer $(\mathbf{4 P b})_{2}$, let alone form stable adducts with the monomer $\mathbf{4 P b}$ in the gas phase, and thus would not improve volatility.

These predictions were then supported by synthetic experiments. Ligands that were synthetically easier to use and ready-to-hand [diethyl ether $\left(\mathrm{Et}_{2} \mathrm{O}\right)$, dimethylethylamine $\left(\mathrm{NMe}_{2} \mathrm{Et}\right), \mathrm{PMe}_{3}$, tmeda, and $\left.\mathrm{PrNHC}\right]$ were mixed with one-half equivalent of $(\mathbf{4 P b})_{2}$ at room temperature in $n$-hexane for two hours, after which time volatiles were removed under vacuum. Except for iPrNHC, unreacted $(\mathbf{4 P b})_{2}$ was recovered quantitatively after mixing with the other ligands and evacuating to dryness. When reacted with iPrNHC, the colour of the solution immediately faded from bright orange to a very pale yellow, which suggested the formation of an adduct. Formation of (4Pb)(iPrNHC) was confirmed by ${ }^{1} \mathrm{H}$ and ${ }^{13} \mathrm{C}$ NMR spectroscopy, which showed the NHC did in fact bind to monomeric $\mathbf{4 P b}$ in solution. However, the other ligands were gases under vacuum at room temperature and the NHC was a non-volatile solid, so it could have been that a solid mixture of iPrNHC and unreacted $(\mathbf{4 P b})_{2}$ was left after removal of solvent. Precisely why only the NHCs were strongly bound to the $\mathrm{Pb}(\mathrm{II})$ center lies outside the scope of this work, but a preliminary bond order and topological analysis showed the Mayer bond order (MBO), Wiberg bond index (WBI), electron density $[\rho(\mathbf{r})]$, and its Laplacian $\left[-\nabla^{2} \rho(\mathbf{r})\right]$ of the $4 \mathbf{P b} \leftarrow \mathbf{L}$ bonds were all smaller than the $\mathrm{Pb}-\mathrm{N}^{*}$ bonds of $(\mathbf{4 P b})_{2}$, except for the NHCs (Table 4.2). Unfortunately, the complex (4Pb)(iPrNHC) darkened during attempted recrystallization from toluene at $-49{ }^{\circ} \mathrm{C}$, presumably due to elimination of $\mathrm{Pb}(0)$, although the decomposition products have not been identified. The thermal instability of NHC adduct and the weak binding of other kinds of ligands led us to abandon this strategy and move onto another for the improvement of $(\mathbf{4 P b})_{2}$. 


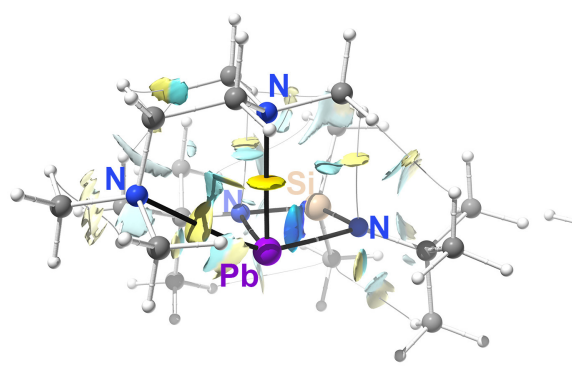

$(4 \mathrm{~Pb})($ tmeda)

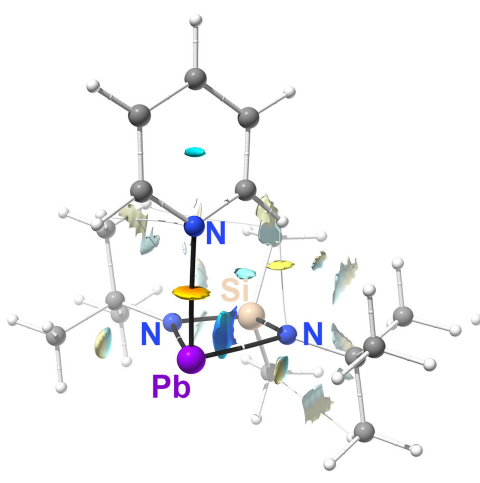

$(4 \mathrm{~Pb})(\mathrm{pyr})$

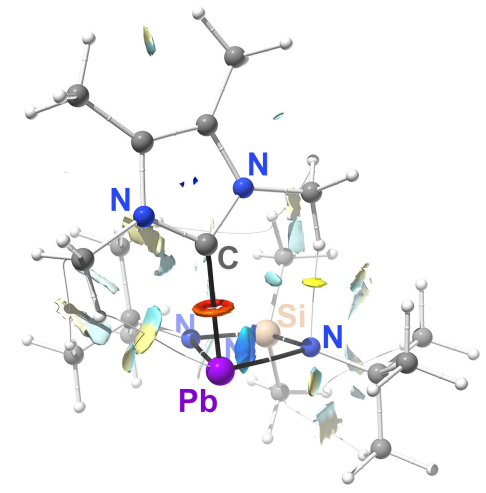

$(4 \mathrm{~Pb})(\mathrm{MeNHC})$

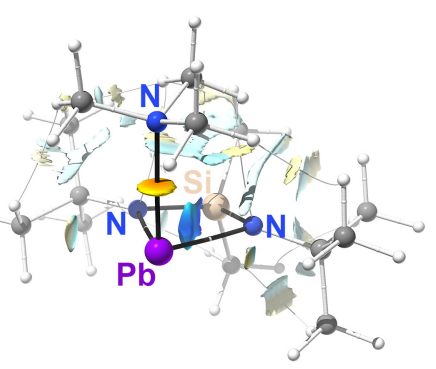

$(4 \mathrm{~Pb})\left(\mathrm{NMe}_{3}\right)$

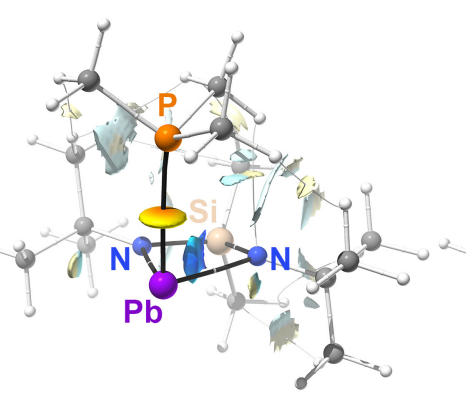

$(4 \mathrm{~Pb})\left(\mathrm{PMe}_{3}\right)$

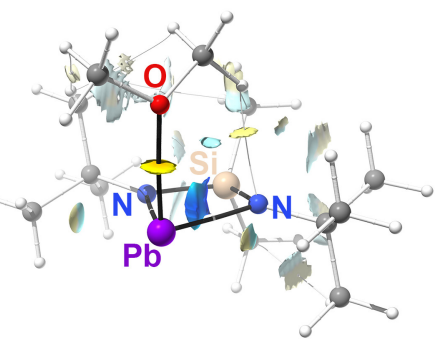

$(4 \mathrm{~Pb})\left(\mathrm{OMe}_{2}\right)$

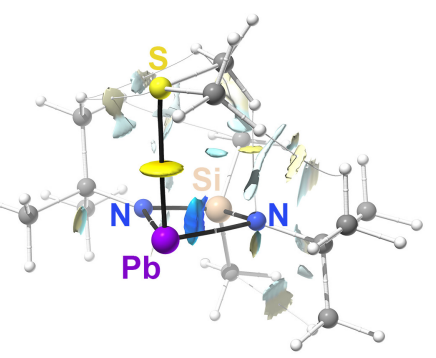

$(4 \mathrm{~Pb})\left(\mathrm{SMe}_{2}\right)$

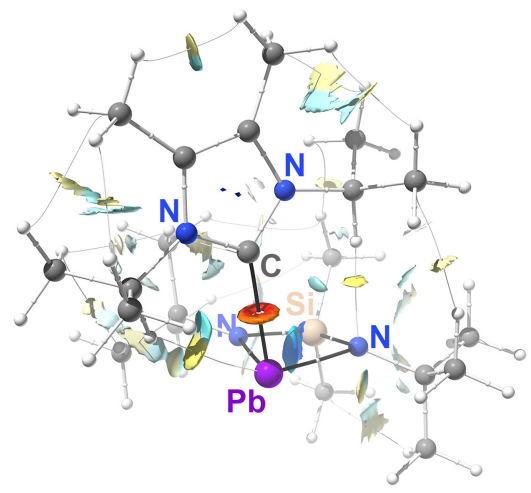

(4Pb)(iPrNHC)

Figure 4.10: Atoms-in-molecules (QTAIM) graphs and non-covalent interaction (NCI) analysis of $(\mathbf{4 P b})(\mathbf{L})$ adducts calculated with DFT [ZORA-def2-QZVPP(SARC-ZORA-TZVPP)//revPBE/ZORAdef2-SVP(SARC-ZORA-TZVP)] in the gas-phase. Atom interaction lines (AILs) connecting $(+3,-3)$ critical points (i.e., nuclei) through $(+3,-1)$ bond critical points (white spheres along AILs) are drawn black if terminating at $\mathrm{Pb}$ and white otherwise. Ring and cage critical points are omitted for clarity. Reduced density gradient (RDG) surfaces are drawn at $0.333 \mathrm{au}$ and their colour is mapped to the values of $\operatorname{sign}\left(\lambda_{2}\right) \rho$. 


\subsubsection{Pendant Tertiary Amines}

We speculated that the addition of a pendant tertiary amine group onto the ligand of $\mathbf{4 P b}$ would lower the electrophilicity of the $\mathrm{Pb}(\mathrm{II})$ center, thus weakening the intermolecular $\mathrm{Pb}-\mathrm{N}^{*}$ bonds enough to destabilize the dimer. Alternatively, two pendant amines could saturate the coordination sphere of $\mathrm{Pb}(\mathrm{II})$ and block dimerization all together. Such a ligand had not yet been reported in the literature, so it was necessary to design one that had tertiary carbons on both amide nitrogens to avoid reductive $\beta$-hydrogen elimination.

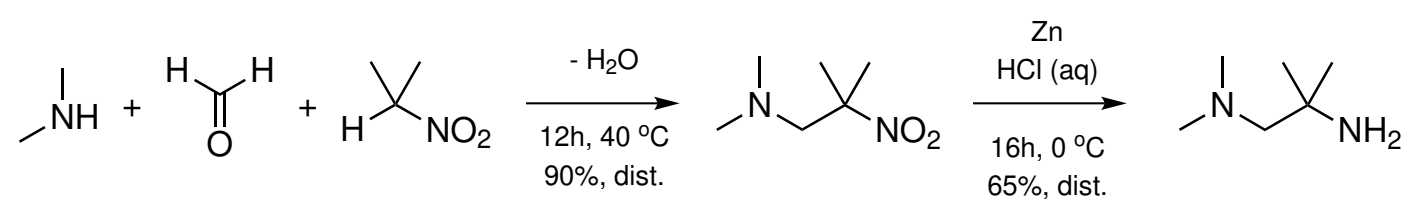

Figure 4.11: Nitro-Mannich reaction and subsequent aqueous metal reduction of the nitroamine to prepare 2-methyl- $N^{\prime}, N^{\prime}$-dimethyl-2,3-diaminopropane $\left(\mathrm{H}_{2} \mathrm{NCMe}_{2} \mathrm{CH}_{2} \mathrm{NMe}_{2}\right)$.

A convenient and high-yield nitro-Mannich reaction followed by reduction furnished the starting material 2-methyl- $N^{\prime}, N^{\prime}$-dimethyl-2,3-diaminopropane $\left(\mathrm{H}_{2} \mathrm{NCMe}_{2} \mathrm{CH}_{2} \mathrm{NMe}_{2}\right)$ in good yield. ${ }^{280}$ It is notable that other alkyl groups may be installed by changing the starting secondary amine (e.g., diethylamine) or nitroalkane (e.g., 3-nitropentane) used in the nitro-Mannich reaction. ${ }^{281}$ Organosilicon chemistry's flexibility then made the synthesis pathways for the rest of the ligands straightforward. Two pendant amines could be installed by reacting two equivalents of $\mathrm{H}(\mathrm{Li}) \mathrm{NCMe}_{2} \mathrm{CH}_{2} \mathrm{NMe}_{2}$ with $\mathrm{Me}_{2} \mathrm{SiCl}_{2}$ to yield $N, N^{\prime}$-bis(1-(dimethylamino)-2-methylpropan-2-yl)-1,1-dimethyl-silanediamine. To install only one pendant amine, the free diamine used for $4 \mathbf{P b},\left[\left(\mathrm{NH}^{t} \mathrm{Bu}\right)_{2} \mathrm{SiMe}_{2}\right]$, can be reacted with one equivalent of $\mathrm{Me}_{2} \mathrm{SiCl}_{2}$ to preform the chloraminosilane $\mathrm{Me}_{2} \mathrm{SiCl}\left(\mathrm{NH}^{t} \mathrm{Bu}\right)$, and then with the lithiated $\mathrm{H}(\mathrm{Li}) \mathrm{NCMe}_{2} \mathrm{CH}_{2} \mathrm{NMe}_{2}$ to yield $N$-tert-butyl- $N^{\prime}$-(1-(dimethylamino)-2-methylpropan-2-yl)-1,1-dimethylsilanediamine. Besides $\mathrm{Pb}(\mathrm{II})$, we believe a lowcost family of bulky chelating ligands with flexible syntheses will find use among otherelement-containing precursors and elsewhere. 


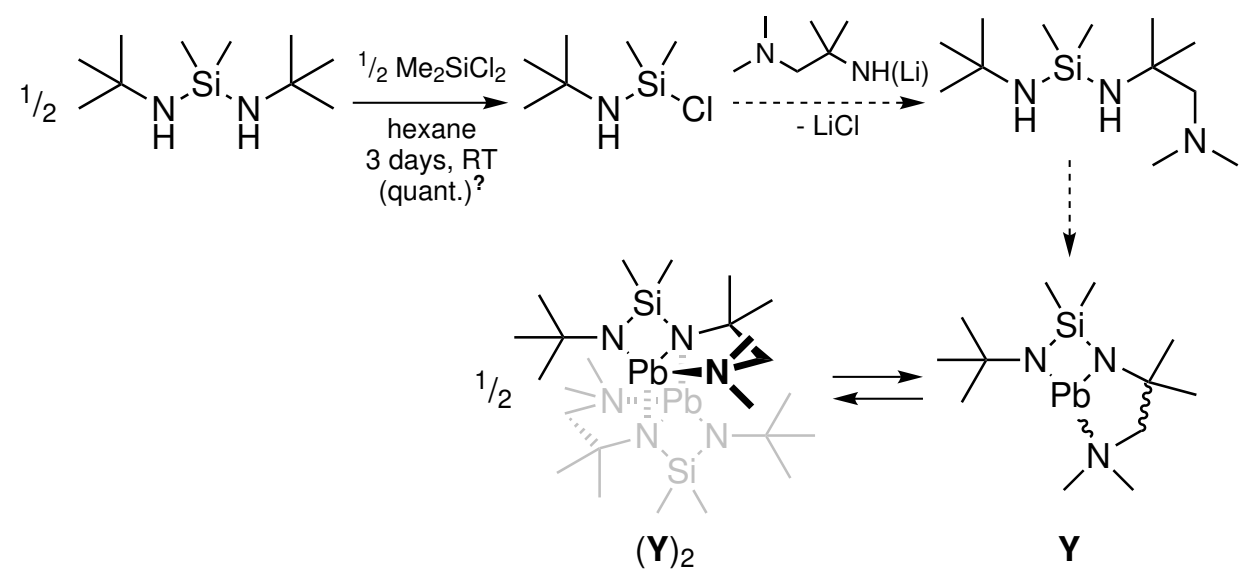

Figure 4.12: Proposed synthesis of $\mathbf{Y}$ and its equilibrium with its $\operatorname{dimer}(\mathbf{Y})_{2}$.

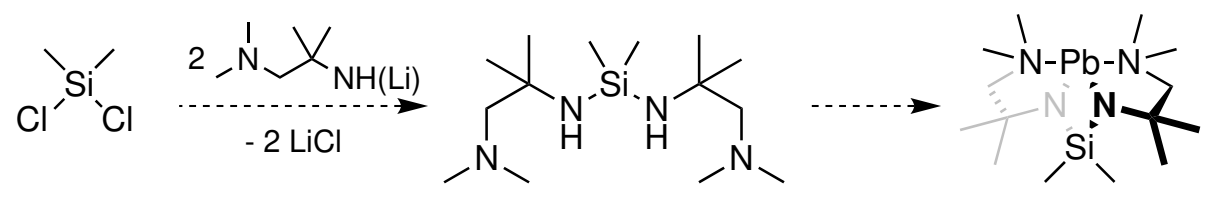

Figure 4.13: Proposed synthesis of $\mathbf{X}$.
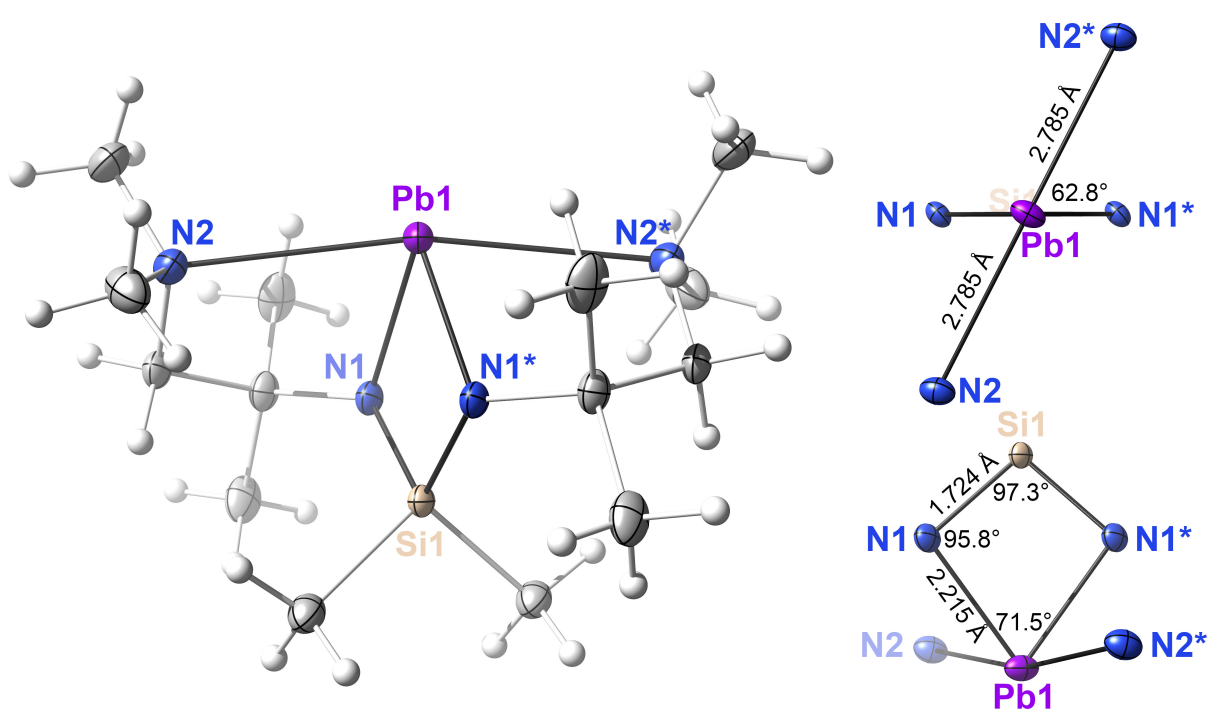

Figure 4.14: Structure of $X$ from single-crystal $X$-ray diffraction (left), and geometric quantities of the amino (top-right) and amido (bottom-right) coordination with carbon and hydrogen omitted for clarity. Only one enantiomer present in the crystal is shown. Ellipsoids are drawn at $50 \%$ and hydrogens are drawn as spheres. 
We intended to install one pendant amine first. Despite the relative simplicity of the ligand synthesis, one key step was problematic: the formation of $\mathrm{Me}_{2} \mathrm{SiCl}\left(\mathrm{NH}^{t} \mathrm{Bu}\right)$ was particularly sluggish and was reported to take three days to complete. In our hands it was still not completed after such time, and we obtained a colourless liquid after vacuum distillation $\left(50{ }^{\circ} \mathrm{C} / 40 \mathrm{mTorr}\right)$ that appeared by ${ }^{1} \mathrm{H}$ NMR spectroscopy to be our target ligand. We then attempted transamination with $\mathbf{0 P b}$ in toluene with heating, which yielded small amounts of a reddish-orange paste after filtration and removal of volatiles under vacuum. Yellow crystals could be sublimed out of the crude mixture $\left(70{ }^{\circ} \mathrm{C} / 40 \mathrm{mTorr}\right)$, but they were invariably contaminated with free ligand. The apparent lower melting point and reasonable volatility of the unidentified compound were encouraging, so we attempted to isolate it by recrystallization. Large yellow single-crystals suitable for X-ray diffraction were obtained after slow cooling of a heptane solution. Crystallography revealed we had isolated a crystal of complex $\mathbf{X}$, the plumbylene with two pendant amines installed on the ligand. It was monomeric in the solid state with a saturated coordination sphere around $\mathrm{Pb}(\mathrm{II})$ including two amido (2.215(2) $\AA$ ) and two amino (2.785(2) $\AA$ ) $\mathrm{Pb}-\mathrm{N}$ bonds. TGA of the recrystallized material showed that it had slightly worse volatility than $(\mathbf{4 P b})_{2}$ $\left(T_{V}=116^{\circ} \mathrm{C}\right)$ despite being a monomer.

Due to the pandemic shutdown and time constraints, we could not properly isolate and characterize the two complexes proposed above to continue our experimental examination and relied on theory to predict whether installation of one pendant amine would improve volatility. While it was expected that two pendant amines would form a monomeric complex, it was not obvious whether one would be enough to destabilize the dimer. Presumably, the additional amino coordination would increase the electron density on the $\mathrm{Pb}(\mathrm{II})$ centers, reducing their electrophilicity, thus weakening possible $\mathrm{Pb}-\mathrm{N}^{*}$ intermolecular coordination. This was supported qualitatively and quantitatively by our calculations on the hypothetical complex $\mathbf{Y}$ and its dimer $(\mathbf{Y})_{2}$ (Figure 4.15). The DFToptimized geometry of $\mathbf{X}$ closely resembled the experimental X-ray structure, so we were 


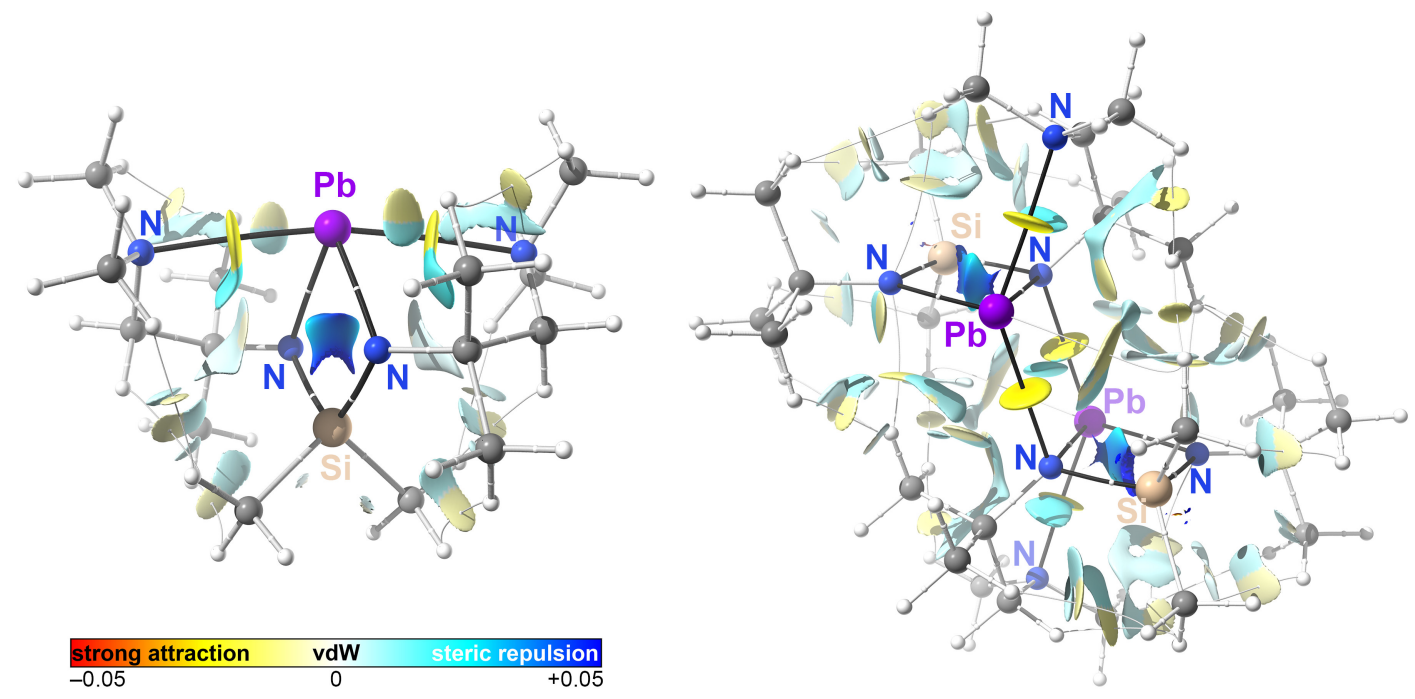

Figure 4.15: Atoms-in-molecules (AIM) graphs and non-covalent interaction (NCI) analysis of complex $\mathbf{X}$ (left) and the stable endo conformer of dimeric $(\mathbf{Y})_{2}$ (right) calculated with DFT (def2QZVPP//revPBE/def2-SVP) in the gas-phase. Atom interaction lines connecting $(+3,-3)$ critical points (i.e., nuclei) through $(+3,-1)$ bond critical points (white spheres) are drawn black if terminating at $\mathrm{Pb}$ and white otherwise. Ring and cage critical points are omitted for clarity. Reduced density gradient $(\mathrm{RDG})$ surface is drawn at a contour value of $0.333 \mathrm{a} \mathrm{u}$ and its colour is mapped to the value of $\operatorname{sign}\left(\lambda_{2}\right) \rho$.

confident that the geometries of $(\mathbf{Y})_{2}$ and its monomer $\mathbf{Y}$ were reasonable, too. The pendant amino $\mathrm{Pb}-\mathrm{N}$ coordination was similar in all three molecules, showing weak but significant non-covalent bonding to the $\mathrm{Pb}(\mathrm{II})$ center. On the other hand, intermolecular non-covalent interactions (NCIs) in $(\mathbf{Y})_{2}$ were obviously weaker than in $(\mathbf{4 P b})_{2}$. This was reflected in the free energies $\Delta G_{\mathrm{D}}^{\diamond}$ and enthalpies $\Delta H_{\mathrm{D}}^{\circ}$ of dissociation for the three possible conformations: where the pendant amino group is on the endo- or exo-cyclic nitrogen, or a mix of the two. Dimerization was only favourable for the endo conformer, probably because the $2 \mathrm{sp}^{3}$-hybridized lone pair was directed away from the $\mathrm{Pb}-\mathrm{N}^{*}$ vector in the endo conformer rather than along it in the exo and mix conformers. The low dissociation energies meant it was likely that it would exist as a monomer during evaporation. However, we still do not know which other factors affect the volatility of these plumbylenes, so it remains an open question whether the dimer $(\mathbf{Y})_{2}$ or its monomer $\mathbf{Y}$ would be more volatile than $(\mathbf{4 P b})_{2}$. 


\subsubsection{Towards a Speculative Precursor Design Process}

The good volatility of $(\mathbf{4 P b})_{2}$ seemed to contradict our understanding of precursor design. It was heavy (815.19 $\left.\mathrm{g} \mathrm{mol}^{-1}\right)$ and evaporated mostly as a dimer. To the best of our knowledge, ligands are found to make volatile metal complexes by trial-and-error, and no general design strategy has emerged in the literature or from industry. Several families of ligands (e.g., halides, alkyls, alkoxides, $\beta$-diketonates, amides, amidinates, etc.) have been used over the history of CVD and ALD for metals across the periodic table, with their suitability for ALD being largely hinged on their volatility. ${ }^{68,70}$ Without a way to predict volatility, new ligands require time-consuming experimentation for each metalligand combination. If the relevant properties (i.e., volatility, melting point, thermal stability) could be predicted theoretically, then significant amounts of time, money, and material could be spared. These properties are generally determined by intermolecular forces, which are dominated by non-covalent interactions that are electrostatic in nature. ${ }^{282-285}$ The development of a quantum chemical method for arbitrary molecules would be highly beneficial for precursor design due to the scarcity of reliable vapour pressure data and the inherent variety of metal-ligand combinations. Here we will describe a project that could potentially solve this problem, and then apply some of its principles qualitatively to the complexes discussed above to predict their relative volatilities.

The general interaction properties function (GIPF) of Murray and Politzer represents

a particularly attractive approach to predicting volatility. ${ }^{282-284,286,287}$ Analytical relationships can be obtained between macroscopic physicochemical properties and the statistical properties of the molecular electrostatic potential (MEP) at the van der Waals (vdW) surface. The MEP at the vdW surface is what other molecules "see" when electrostatically interacting together, and it is closely linked to NCIs and the physicochemical properties we wish to understand. Since the MEP is entirely determined computationally, the GIPF approach can be used to predict the properties of molecules that have not yet been synthe- 
sized. To our knowledge, only two GIPFs for normal boiling points have been published: the original by Murray et al. in 1993 (FBP93, Equation 4.1), ${ }^{286}$ and a reworked version by Wang et al. in 2017 (FBP17, Equation 4.2). ${ }^{287}$

$$
\begin{array}{r}
\operatorname{FBP} 93\left(A_{\mathrm{vdW}}, v \sigma_{ \pm}^{2}\right)=2.736 A_{\mathrm{vdW}}+33.31 \sqrt{v \sigma_{ \pm}^{2}}-72.05 \\
\operatorname{FBP} 17\left(1 / A_{\mathrm{vdW}}, \Pi^{2}, \sqrt[3]{\eta}\right)=125.3 \Pi^{2}-\frac{143.2}{A_{\mathrm{vdW}}}+\frac{194.7}{\sqrt[3]{\eta}}+27.0
\end{array}
$$

where $A_{\mathrm{vdW}}$ is the van der Waals surface area of a molecule $[\rho(\mathbf{r})=0.001 \mathrm{a} \mathrm{u}] ; \sigma_{ \pm}^{2}$ is the total variance of the MEP; $v$ is the balance parameter of the total variance; $\Pi$ is the standard deviation of the MEP, also called the internal charge separation; and $\eta$ is the chemical hardness, as defined by Koopmans's theorem.

$$
\begin{aligned}
\sigma_{ \pm}^{2}=\sigma_{+}^{2}+\sigma_{-}^{2} & =\frac{1}{m} \sum_{i=1}^{m}\left[V^{+}\left(\mathbf{r}_{i}\right)-\bar{V}_{s}^{+}\right]^{2}+\frac{1}{n} \sum_{i=1}^{n}\left[V^{-}\left(\mathbf{r}_{i}\right)-\bar{V}_{s}^{-}\right]^{2} \\
v & =\frac{\sigma_{+}^{2} \sigma_{-}^{2}}{\left[\sigma_{ \pm}^{2}\right]^{2}} \\
\Pi & =\frac{1}{n+m} \sum_{i=1}^{n+m}\left|V_{s}\left(\mathbf{r}_{i}\right)-\bar{V}_{s}\right| \\
\eta & =\frac{E_{\mathrm{LUMO}}-E_{\mathrm{HOMO}}}{2}
\end{aligned}
$$

where $\sigma_{+}^{2}$ and $\sigma_{-}^{2}$ are the positive and negative variances, respectively; $V^{+}\left(\mathbf{r}_{i}\right)$ and $V^{-}\left(\mathbf{r}_{i}\right)$ are the positive and negative values of the MEP $V(\mathbf{r}) ; \bar{V}_{s}^{+}$and $\bar{V}_{s}^{-}$are their averages; and $E_{\mathrm{LUMO}}$ and $E_{\mathrm{HOMO}}$ are the lowest-unoccupied and highest-occupied molecular orbital energies.

For a training set of 99 organic molecules, the correlation of FBP93 was impressive $\left(R^{2}=0.943\right)$ considering the MEPs were computed at very low levels of theory (STO$\left.5 \mathrm{G}^{*} / / \mathrm{HF} / \mathrm{STO}-3 \mathrm{G}\right)$, still the standard deviation was large $(s=36.5 \mathrm{~K})$. Wang et al. developed FBP17 to theoretically evaluate low-global-warming-potential replacements for the dielectric gas $\mathrm{SF}_{6}$. They found FBP93 performed very poorly for their training set 
$\left(R^{2} \approx 0.7\right)$ which included exotic and inorganic molecules (e.g., FOF, $\left.\mathrm{N}_{2} \mathrm{~F}_{4}, \mathrm{CF}_{3} \mathrm{SO}_{2} \mathrm{~F}\right)$, so they reparameterized FBP17 to better reflect the effects of NCIs on gaseous molecules from the outset. First, they performed more accurate DFT calculations [M06-2X/6-31++G(d,p)]; their training set was expanded to account for extreme or unusual effects of fluorination; the internal charge separation $\Pi$ was used instead of the balanced total variance $v \sigma_{ \pm}^{2}$ as it was argued that it better represents the averaged effect of the MEP on NCIs when the molecule is rotating freely as a gas; and finally the chemical hardness $\eta$ was included to account for the relative strengths of induced dipole interactions. This resulted in a much better correlation $\left(R^{2}=0.985\right)$ and a much narrower standard deviation $(s=8 \mathrm{~K})$. The chemical hardness $\eta$ term accounted for about half of the value of FBP17, signalling the importance of the internal electronic structure alongside the MEP surface properties. Unfortunately, the vast majority of CVD and ALD precursors are not volatile enough for normal boiling points to be measured, so of neither the FBP93 nor FBP17 were applicable here. Although the development of a GIPF for the vapour pressures of CVD and ALD precursors lies outside the scope of this work, we nevertheless used similar methods to qualitatively examine the plumbylenes discussed above.

Table 4.3: Vapour pressure and calculated MEP properties of known plumbylenes. ${ }^{a, b}$

\begin{tabular}{cccccccc}
\hline Complex & $\begin{array}{c}T_{V} \\
{ }^{\circ} \mathrm{C}\end{array}$ & $\begin{array}{c}A_{\mathrm{vdW}} \\
\AA^{2}\end{array}$ & $\begin{array}{c}A_{\text {polar }} \\
\AA^{2}\end{array}$ & $\begin{array}{c}\sigma_{ \pm}^{2} \\
\mathrm{kcal}^{2} / \mathrm{mol}^{2}\end{array}$ & & $\begin{array}{c}\Pi \\
\mathrm{kcal} / \mathrm{mol}\end{array}$ & $\begin{array}{c}\eta \\
\text { a.u. }\end{array}$ \\
\hline $\mathrm{Pb}(\mathrm{tmhd})_{2}$ & 166 & 441.10 & 63.09 & 41.46 & 0.195 & 5.196 & 0.1728 \\
$\mathrm{~Pb}(\text { dmamp })_{2}$ & $\mathbf{-}^{\mathrm{c}}$ & 316.56 & 66.32 & 69.69 & 0.158 & 6.561 & 0.1544 \\
$\mathrm{~Pb}(\text { dedtc })_{2}$ & 265 & 362.38 & 170.25 & 73.54 & 0.242 & 9.642 & 0.1554 \\
$\mathbf{0 P b}$ & 127 & 402.42 & 31.04 & 22.86 & 0.145 & 4.410 & 0.1463 \\
$\mathbf{1 P b}$ & 94 & 294.43 & 12.56 & 18.14 & 0.174 & 3.764 & 0.1411 \\
$(\mathbf{4 P b})_{2}$ & 112 & 449.22 & 32.20 & 20.56 & 0.249 & 3.886 & 0.1488 \\
$\left(\mathbf{4 P b} \mathbf{P b e}_{2}\right)_{2}$ & 116 & 495.41 & 30.21 & 20.29 & 0.248 & 3.845 & 0.1492 \\
\hline
\end{tabular}

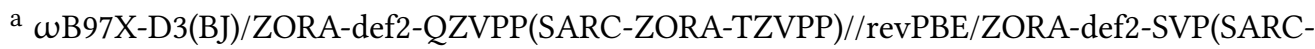
ZORA-TZVP)

${ }^{\mathrm{b}}$ MEP properties were calculated at the van der Waals surface $[\rho(\mathbf{r})=0.001 \mathrm{au}]$.

${ }^{c}$ The 1 Torr vapour pressure temperature of $\mathrm{Pb}$ (dmamp $)_{2}$ has not been measured by us. It was previously reported to reach 1.97 Torr at $126{ }^{\circ} \mathrm{C},{ }^{250}$ but the authors did not report how this was measured. 

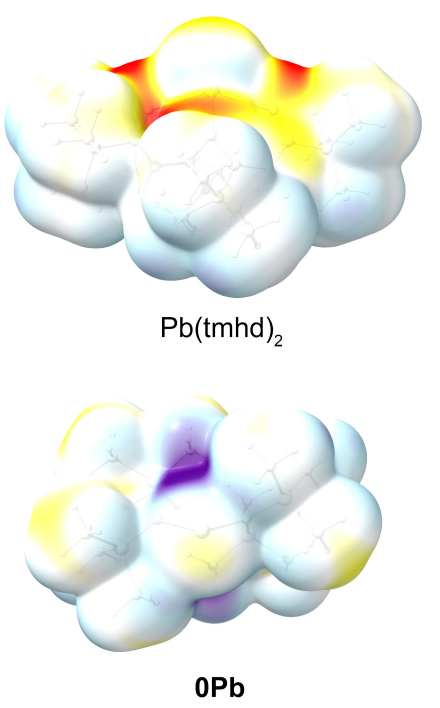

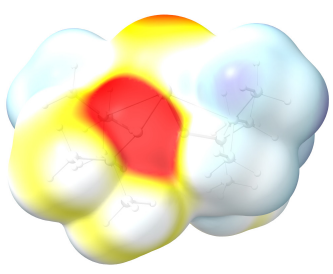

$\mathrm{Pb}(\mathrm{dmamp})_{2}$

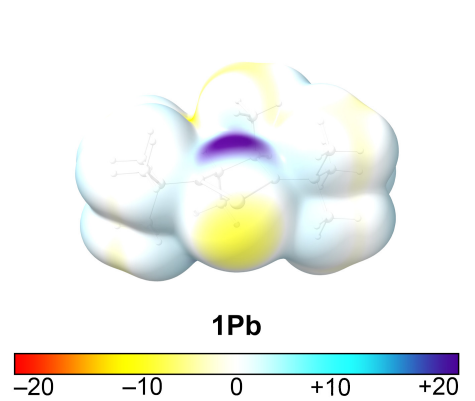

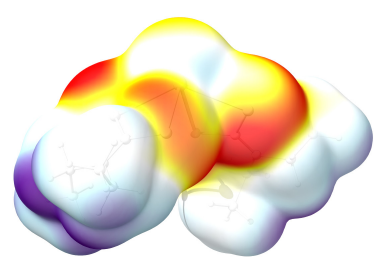

$\mathrm{Pb}(\text { dedtc) })_{2}$

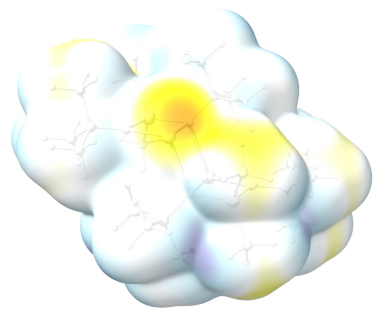

$(4 \mathrm{~Pb})_{2}$

Figure 4.16: Molecular electrostatic potential (MEP, in $\mathrm{kcal} / \mathrm{mol}$ ) at the van der Waals surface $(\rho(\mathbf{r})=0.001 \mathrm{a} \mathrm{u})$ of known Pb-containing ALD precursors and $(\mathbf{4} \mathbf{P b})_{2}$. Positive potentials are in cyan to indigo, while negative potentials are in yellow to red. Positive (cyan to indigo) regions correspond to Lewis acidic sites, and negative (yellow to red) regions correspond to Lewis basic sites, respectively.

Relevant GIPF descriptors were obtained from DFT for plumbylenes with known vapour pressures ( $\omega$ B97X-D3(BJ)/def2-QZVPP//revPBE/def2-SVP, Table 4.3), and we visualized the MEP surface to see how it correlated to chemical features of the molecules (Figure 4.16). The differences between the older $\mathrm{Pb}$-containing precursors and our diamido plumbylenes were obvious. Despite being coordinately saturated, $\mathrm{Pb}(\mathrm{tmhd})_{2}, \mathrm{~Pb}(\mathrm{dmamp})_{2}$, and especially lead(II) bis( $N, N$-diethyldithiocarbamate) $\mathrm{Pb}$ (dedtc) $)_{2}$ had large positive and negative areas on their MEP surfaces (polar surface area > 10\%), while diamido plumbylenes were mostly non-polar (polar surface area $<10 \%$ ). There was a strong correlation between the absolute polar surface area and the 1 Torr temperature (Equation $4.7, R^{2}=0.964$ ), but no correlation with the total surface area $A_{\mathrm{vdW}}\left(R^{2}=0.0154\right)$. Although complexes $\mathbf{0 P b}$ and $\mathbf{1 P b}$ had positive patches on their MEPs associated with their empty $6 \mathrm{p}_{z}^{0}$ orbitals, they did not have the corresponding negative areas to form attractive electrostatic interactions. Contrary to FBP17, we found no correlation between 1 Torr temperature and chemical hardness $\eta\left(R^{2}=0.236\right)$, while the total variance $\sigma_{ \pm}^{2}$ had a slightly better cor- 
relation (Equation 4.8, $R^{2}=0.987$ ) than the internal charge separation $\Pi$ (Equation 4.9, $\left.R^{2}=0.970\right)$.

$$
\begin{gathered}
T_{V}\left(A_{\text {polar }}\right)=1.090 A_{\text {polar }}+85.00 \\
T_{V}\left(\sigma_{ \pm}^{2}\right)=2.909 \sigma_{ \pm}^{2}+51.22 \\
T_{V}(\Pi)=27.96 \Pi+3.414
\end{gathered}
$$

Table 4.4: Calculated MEP properties of hypothetical plumbylenes. ${ }^{\mathrm{a}, \mathrm{b}}$

\begin{tabular}{ccccccc}
\hline Complex & $\begin{array}{c}A_{\mathrm{vdW}} \\
\AA^{2}\end{array}$ & $\begin{array}{c}A_{\text {polar }} \\
\AA^{2}\end{array}$ & $\begin{array}{c}\sigma_{ \pm}^{2} \\
\mathrm{kcal}^{2} / \mathrm{mol}^{2}\end{array}$ & & $\begin{array}{c}\Pi \\
\mathrm{kcal} / \mathrm{mol}\end{array}$ & $\begin{array}{c}\eta \\
\text { a.u. }\end{array}$ \\
\hline$\left(\mathbf{4} \mathbf{P b}_{\mathrm{Cyb}}\right)_{2}$ & 466.18 & 29.51 & 18.66 & 0.250 & 3.781 & 0.1501 \\
$\left(\mathbf{4} \mathbf{P b}_{\text {btbas }}\right)_{2}$ & 423.44 & 30.99 & 20.96 & 0.249 & 4.028 & 0.1505 \\
$\mathbf{X}$ & 378.47 & 43.35 & 24.85 & 0.242 & 5.071 & 0.1485 \\
$\mathbf{Y}$ & 324.54 & 85.90 & 49.47 & 0.248 & 6.419 & 0.1553 \\
$(\mathbf{Y})_{2}$ & 511.15 & 58.80 & 21.98 & 0.250 & 4.913 & 0.1450 \\
\hline
\end{tabular}

a $\omega B$ 97X-D3(BJ)/ZORA-def2-QZVPP(SARC-ZORA-TZVPP)//revPBE/ZORA-def2SVP(SARC-ZORA-TZVP)]

${ }^{\mathrm{b}}$ MEP properties were calculated at the van der Waals surface $[\rho(\mathbf{r})=0.001 \mathrm{a} \mathrm{u}]$.

The relative differences among the diamido plumbylenes were less trivial to deduce. They all have low absolute and fractional polar surface area, small total variance $\sigma_{ \pm}^{2}$, and small internal charge separation $\Pi$. The very high volatility of $\mathbf{1 P b}$ was likely a consequence of it having the smallest of all these values. Complexes $\mathbf{0 P b},(\mathbf{4 P b})_{2}$, and $\left(\mathbf{4} \mathbf{P} \mathbf{b}_{\mathrm{tPe}}\right)_{2}$ did not have obvious differences to explain their relative volatilities. But the 1 Torr vapour pressure temperatures were all very similar among the diamido plumbylenes, so it was unlikely that we could unambiguously sort the volatilities of such similar complexes without a very precise model (e.g., the standard deviation of FBP17 was $8^{\circ} \mathrm{C}$ ). Nevertheless, we believe these data can at least be used to justify further experimental studies on potential hypothetical complexes (Table 4.4). 


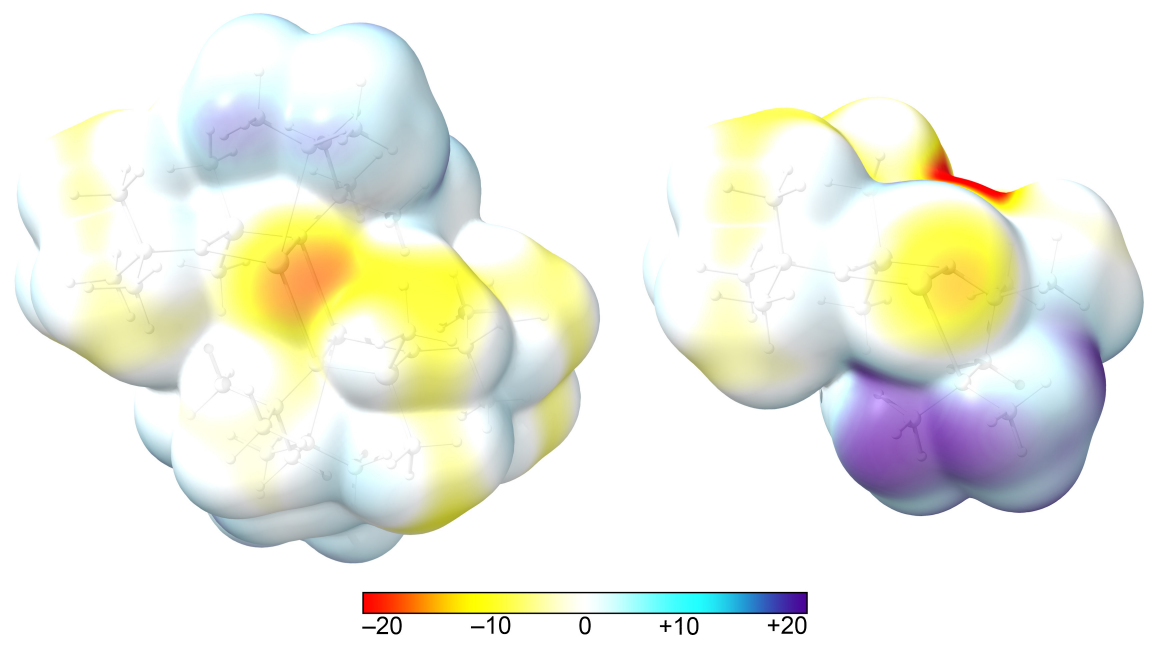

Figure 4.17: Molecular electrostatic potential (MEP, in $\mathrm{kcal} / \mathrm{mol}$ ) at the van der Waals surface $(\rho(\mathbf{r})=0.001 \mathrm{a} \mathrm{u})$ of the $\operatorname{dimer}(\mathbf{Y})_{2}$ and its monomer $\mathbf{Y}$.

In particular, we can draw the following conclusions. Since every property that appears to affect volatility is greater in $\mathbf{X},(\mathbf{Y})_{2}$, and $\mathbf{Y}$ than in $(\mathbf{4 P b})_{2}$, it is likely they would be less volatile, and that the additional effort required to prepare them was in vain. This becomes clear

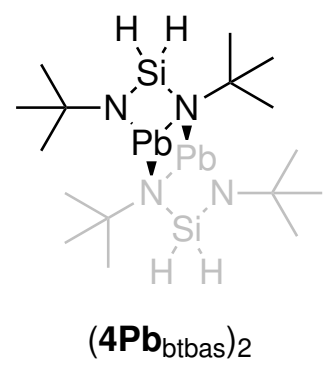
when comparing the dimer $(\mathbf{Y})_{2}$ and the monomer $\mathbf{Y}(\mathrm{Ta}-$ ble 4.4 and Figure 4.17). If the dimer really is destabilized and dissociation to monomers is more favourable, it is unlikely the complex will be more volatile than $\mathbf{1 P b}$ or $(\mathbf{4 P b})_{2}$ because of the greater variation in its MEP surface. Conversely, since dimerization appears to be a feature of $(\mathbf{4 P b})_{2}$ rather than a hindrance, it is possible that $\left(\mathbf{4} \mathbf{P} \mathbf{b}_{\mathrm{Cyb}}\right)_{2}$ and the hydrogen-derivative bis[lead(II) $N, N^{\prime}$-bis(tert-butylamido)silane] $\left[\left(\mathbf{4} \mathbf{P b}_{\text {btbas }}\right)_{2}\right]$ could be more volatile considering their MEP properties are equal-to or less-than those of $(\mathbf{4 P b})_{2}$. The hydrogen-derivative is especially interesting as its ligand is the popular Si-containing ALD precursor bis(tert-butylamino)silane $\left[\left({ }^{t} \mathrm{BuNH}\right)_{2} \mathrm{SiH}_{2}\right.$, btbas $],{ }^{288}$ and is already available in large quantities with electronics-grade purity. It is important to keep in mind the tiny sample size $(N=6)$ of this example set. Any attempt at a quantitative analysis with such a small sample size would not be representative and insight using it would be 
misguided. Future attempts to develop a GIPF for vapour pressures must first compile a much larger and more diverse training set for a multivariate regression, while here we only performed crude univariate regressions.

There are several deficiencies to the quantum chemical calculations used here and in the development of FBP93 and FBP17: the effect of functional and basis set on the MEP has not been benchmarked; the density envelope arbitrarily chosen at $\rho(\mathbf{r})=0.001 \mathrm{au}$ encloses $97 \%$ of the electron density of hydrocarbons, but it may be insufficient for metal complexes; orbital energies from DFT are inaccurate, so the chemical hardness $\eta$ derived from these may not be trustworthy; and, finally, different conformations have not been taken into account. In the development of a GIPF to predict vapour pressures of CVD and ALD precursors, the following should be considered: high-level ab initio densities (e.g., DLPNO-CCSD) should be used to eliminate unknown inaccuracies introduced by DFT approximations; the density envelope for representative metal-containing complexes should be examined to determine an appropriate value for the van der Waals surface; chemical hardness $\eta$ should be calculated from the ionization potential (IA) and electron affinity (EA) by calculating the relative energies of the cation and anion, rather than relying on the orbital energies; and a conformer search should be performed.

\subsection{Conclusions}

Four-membered 1,3-diaza-2-plumba-4-silacyclobutanes like bis[lead(II) $N, N^{\prime}$-di-tert-butyl1,1-dimethylsilanediamide $]\left[(\mathbf{4 P b})_{2}\right]$ are volatile and thermally stable molecules with potential to be new low- and high-temperature ALD precursors for $\mathrm{Pb}$-containing materials. We attempted to produce a more volatile derivative by lowering the melting point with bulkier ligands, forming adducts with ancillary ligands, and adding chelating pendant amine groups to make new plumbylenes. Our results suggest none of these approaches

worked, and we were unable to complete the experiments due to the pandemic. Rely- 
ing on theoretical methods, we attempted to understand why the modifications did not produce more volatile precursors. At the same time, we began exploring the molecular electrostatic potential (MEP) of real and hypothetical plumbylenes to understand what makes some more volatile than others. Several statistical descriptors derived from quantum chemistry were identified that had a significant influence on volatility, but we did not have a large enough sample size to perform a complete regression analysis to generalize our findings. Using this qualitative analysis, we predict that the plumbylene derived from bis(tert-butylamino)silane (btbas) would likely be a volatile precursor, adding even more value to the popular ALD precursor. Finally, we outlined a future project to develop a general interaction properties function (GIPF) for vapour pressures.

\subsection{Experimental Details}

\subsubsection{Synthesis}

\subsubsection{General Considerations}

All manipulations were performed under air-free conditions using standard Schlenk techniques or in a $\mathrm{N}_{2}$-filled (99.998\% purity) MBraun Labmaster 130 dry-box unless otherwise specified. Vacuum on the Schlenk line was obtained with an Edwards RV12 vacuum pump that achieved a base pressure of 10 mTorr (Hg-filled McLeod gauge) that was controlled between 10-500 mTorr with a Vernier needle valve routed to a spare inert-gas tap. Paraformaldehyde (crystalline, \%, Millipore-Sigma), dimethylamine (40 wt.\% in $\mathrm{H}_{2} \mathrm{O}$, Millipore-Sigma), 2-nitropropane (\%, Millipore-Sigma), zinc (powder, $x$ mesh, 99\%, Strem), dichlorodimethylsilane ( $\geq 99.5 \%$, Millipore-Sigma), tert-butylamine (98\%, Millipore-Sigma), tert-pentylamine (i.e., tert-amylamine, $98 \%{ }^{n} \mathrm{BuLi}$ (2.5 $\mathrm{M}$ in hexanes), dimethylethylamine (Millipore-Sigma), $N, N, N^{\prime}, N^{\prime}$-tetramethylethylene-1,2-diamine (tmeda, ReagentPlus ${ }^{\circledR}$, 99\%), trimethylphosphine (Strem), and lead(II) bis(2,2,6,6-tetramethyl-heptan-3,5-dionate) 
$\left[\mathrm{Pb}(\mathrm{tmhd})_{2}\right.$, Strem $]$ were used as received. Diethyl ether, toluene, hexanes, and pentane were ACS Reagent grade, purified by an MBraun Solvent Purifier System, and stored over $4 \AA$ molecular sieves for more than a day before use. $\mathrm{PbCl}_{2}$ was prepared by the addition of $\mathrm{HCl}(\mathrm{aq})(\mathrm{ca} .6 \mathrm{~N})$ to an ice-cooled solution of $\mathrm{Pb}\left(\mathrm{CH}_{3} \mathrm{CO}_{2}\right)_{2}$ (aq) (Baker, 95\%) until precipitation ceased. The white precipitate was collected by filtration, dried under vacuum with heating $\left(130^{\circ} \mathrm{C} / 10 \mathrm{mTorr}\right)$, and stored in a dry box. $N, N^{\prime}$-di-tert-butyl-1,1dimethyldiaminosilane $\left[\mathrm{Me}_{2} \mathrm{Si}\left(\mathrm{NH}^{t} \mathrm{Bu}\right)_{2}\right],{ }^{289} N, N^{\prime}$-di-tert-butyl- $N$, $N^{\prime}$-dilithio-1,1-dimethyl diamidosilane $\left[\mathrm{Me}_{2} \mathrm{Si}\left(\mathrm{N}(\mathrm{Li}){ }^{t} \mathrm{Bu}\right)_{2}\right],{ }^{290}$ 2-methyl- $N^{\prime}, N^{\prime}$-dimethyl-2,3-diaminopropane, ${ }^{280}$ lead(II) bis[bis(trimethylsilylamide)] (0Pb), ${ }^{182}$ and $N^{1}, N^{3}$-di-iso-propyl-4,5-dimethylimidazol-2ylidene (iPrNHC) ${ }^{291}$ were prepared according to literature procedures.

\subsubsection{Bis[lead(II) $N, N^{\prime}$-di-tert-butyl-1,1-dimethylsilanediamide] [(4Pb $\left.)_{2}\right]$}

Bis[lead(II) $N, N^{\prime}$-di-tert-butyl-1,1-dimethylsilanediamide] $\left[(\mathbf{4 P b})_{2}\right]$ was prepared according to a modified literature procedure. ${ }^{260}$ Dilithio diamide $\mathrm{Me}_{2} \mathrm{Si}\left[\mathrm{N}(\mathrm{Li})^{t} \mathrm{Bu}\right]_{2}(1$ eq., $7.928 \mathrm{~g}$, $37.00 \mathrm{mmol}$ ), recrystallized from hexane, and a slight excess of $\mathrm{PbCl}_{2}(1.1$ eq., $11.31 \mathrm{~g}$, $40.70 \mathrm{mmol}$ ) were placed in a $250 \mathrm{~mL}$ round-bottom flask with a magnetic stir-bar in a $\mathrm{N}_{2}$-filled dry-box. Then, $100 \mathrm{~mL}$ of hexane and ca. $2 \mathrm{~mL}$ of $\mathrm{Et}_{2} \mathrm{O}$ were added all at once and the flask was closed with a greased glass stopper held with a plastic Keck clip. A slight exotherm occurs as the dilithio salt dissolves and begins to react with $\mathrm{PbCl}_{2}$. The reaction mixture was then stirred overnight (ca. $16 \mathrm{~h}$ ). After the reaction had proceeded, an off-white precipitate developed under a bright orange supernatant liquid which was separated by filtration through a pad of Celite and a medium porosity sintered glass filter to yield a bright orange filtrate. (Note: if crude or in-situ generated $\mathrm{Me}_{2} \mathrm{Si}\left[\mathrm{N}(\mathrm{Li})^{t} \mathrm{Bu}\right]_{2}$ is used, a dark precipitate, presumably contaminated with $\mathrm{Pb}(0)$, may be obtained with a slightly diminished yield.) Solvents and other volatiles are removed under vacuum, and a crude orange solid is obtained. The crude product can be purified by vacuum sublimation $\left(100{ }^{\circ} \mathrm{C} / 40 \mathrm{mTorr}\right)$ into an air-cooled $90^{\circ}$ glass elbow to yield $(\mathbf{4 P b})_{2}$ as yellow ther- 
mochromic crystals $\left(14.04 \mathrm{~g}, 92 \%\right.$ yield based on ligand). M.p. $85^{\circ} \mathrm{C}$, sublimed; lit. $85^{\circ} \mathrm{C}$, recrys. $\mathrm{Et}_{2} \mathrm{O}$. Crystals suitable for X-ray diffraction were grown by slowly cooling a solution of $(\mathbf{4 P b})_{2}$ in toluene overnight at $-49{ }^{\circ} \mathrm{C} .{ }^{1} \mathrm{H}$ NMR $\left(300 \mathrm{MHz}, \mathrm{C}_{6} \mathrm{D}_{6}, \delta_{\mathrm{C}_{6} \mathrm{D}_{5} H}=7.161 \mathrm{ppm}\right)$ : $0.420\left(6 \mathrm{H}, s, \mathrm{Si}\left(\mathrm{CH}_{3}\right)_{2}\right), 1.24\left(18 \mathrm{H}, s, \mathrm{PbN}-\mathrm{C}\left(\mathrm{CH}_{3}\right)_{3}\right)$.

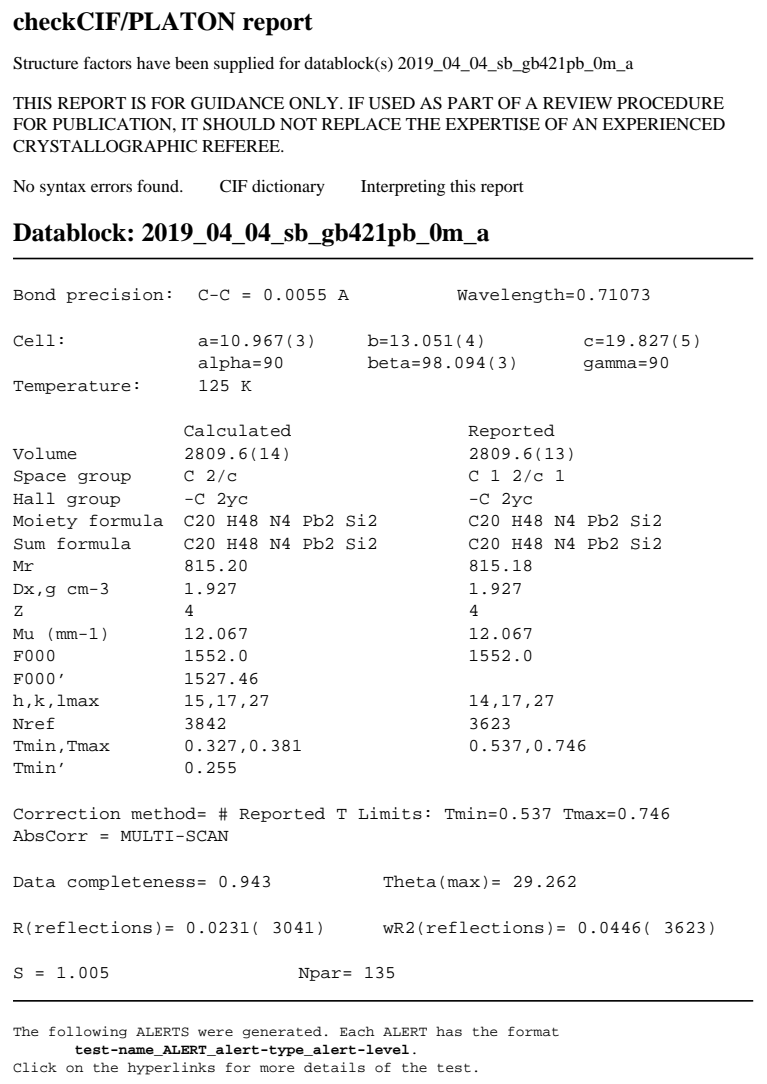

Figure 4.18: CheckCIF report for $(4 \mathrm{~Pb})_{2}$.

\subsubsection{3 $N, N^{\prime}$-di-tert-pentyl-1,1-dimethyldiaminosilane $\left[\mathrm{Me}_{2} \mathrm{Si}\left(\mathrm{NH}^{t} \mathrm{Pe}\right)_{2}\right]$}

The ligand $N, N^{\prime}$-di-tert-pentyl-1,1-dimethyldiaminosilane $\left[\mathrm{Me}_{2} \mathrm{Si}\left(\mathrm{NH}^{t} \mathrm{Pe}\right)_{2}\right]$ was prepared similarly to $\mathrm{Me}_{2} \mathrm{Si}\left(\mathrm{NH}^{t} \mathrm{Bu}\right)_{2} \cdot{ }^{289}$ In a $\mathrm{N}_{2}$-filled dry-box, tert-pentylamine (4 eq., $25.000 \mathrm{~g}$, $286.83 \mathrm{mmol}$ ) was dissolved in $30 \mathrm{~mL}$ hexane and added dropwise to a solution of $\mathrm{Me}_{2} \mathrm{SiCl}_{2}$ (1 eq., $9.255 \mathrm{~g}, 71.70 \mathrm{mmol}$ ) in $100 \mathrm{~mL}$ hexane in a $200 \mathrm{~mL}$ Schlenk flask. A volumi- 
nous white precipitate $\left({ }^{t} \mathrm{PeNH}_{3} \mathrm{Cl}\right)$ developed immediately and the reaction mixture was stirred overnight (ca. $16 \mathrm{~h}$ ) to ensure completion. The next day, the precipitated tertpentylammonium chloride was removed by filtration through a fine sintered glass frit and washed three times with ca. $30 \mathrm{~mL}$ portion of hexane to yield a colourless filtrate. The filtrate was cooled and volatiles were removed under vacuum $\left(0{ }^{\circ} \mathrm{C} / 100 \mathrm{mTorr}\right)$ to yield $\mathrm{Me}_{2} \mathrm{Si}\left(\mathrm{NH}^{t} \mathrm{Pe}\right)_{2}$ as a colourless liquid (13.075 g, $80 \%$ based on $\left.\mathrm{Me}_{2} \mathrm{SiCl}_{2}\right)$. The purity

of the liquid was determined to be satisfactory by ${ }^{1} \mathrm{H}$ NMR spectroscopy and was used without further purification. ${ }^{1} \mathrm{H}$ NMR $\left(300 \mathrm{MHz}, \mathrm{CDCl}_{3}, \delta_{\mathrm{CHCl}_{3}}=7.280 \mathrm{ppm}\right): 0.086(6 \mathrm{H}, s$, $\left.\mathrm{Si}\left(\mathrm{CH}_{3}\right)_{2}\right), 0.526\left(2 \mathrm{H}, b r,\left({ }^{t} \mathrm{Pe}\right) \mathrm{NH}\right), 0.865\left(6 \mathrm{H}, t, \mathrm{NH}-\mathrm{C}\left(\mathrm{CH}_{3}\right)_{2}\left(\mathrm{CH}_{2} \mathrm{CH}_{3}\right),{ }^{3} J=4.5 \mathrm{~Hz}\right), 1.128$ $\left(12 \mathrm{H}, s, \mathrm{NH}-\mathrm{C}\left(\mathrm{CH}_{3}\right)_{2}\left(\mathrm{CH}_{2} \mathrm{CH}_{3}\right)\right), 1.374\left(4 \mathrm{H}, q, \mathrm{NH}-\mathrm{C}\left(\mathrm{CH}_{3}\right)_{2}\left(\mathrm{CH}_{2} \mathrm{CH}_{3}\right)\right)$.

\subsubsection{Bis[lead(II) $N, N^{\prime}$-di-tert-pentyl-1,1-dimethylsilanediamide] $\left[\left(4 \mathrm{~Pb}_{\mathrm{tPe}}\right)_{2}\right]$}

$\mathrm{Me}_{2} \mathrm{Si}\left(\mathrm{NH}^{t} \mathrm{Pe}\right)_{2}(0.500 \mathrm{~g}, 2.17 \mathrm{mmol})$ was dissolved in $30 \mathrm{~mL}$ pentane in a $200 \mathrm{~mL}$ Schlenk flask, tert-butyllithium (2 eq., $2.6 \mathrm{~mL}$ of $1.7 \mathrm{M}$ in pentane, $4.4 \mathrm{mmol}$ ) was added dropwise, and the mixture was stirred for $2 \mathrm{~h}$ at ambient temperature. Then, ca. $2 \mathrm{~mL}$ of $^{2} \mathrm{t}_{2} \mathrm{O}$ was added, solid $\mathrm{PbCl}_{2}$ (1.1 eq., $0.666 \mathrm{~g}, 2.39 \mathrm{mmol}$ ) was added all at once, and the reaction mixture was stirred overnight (ca. $16 \mathrm{~h}$ ). After the reaction had proceeded, a black precipitate developed under a bright orange supernatant liquid which was separated by filtration through a pad of Celite and a medium porosity sintered glass filter to yield a bright orange filtrate. Volatiles were removed under vacuum to yield a crude orange paste. The crude product was purified by vacuum sublimation $\left(80{ }^{\circ} \mathrm{C} / 10 \mathrm{mTorr}\right)$ into an air-cooled $90^{\circ}$ glass elbow to yield $\left(\mathbf{4} \mathbf{P} \mathbf{b}_{\mathrm{tPe}}\right)_{2}$ as prismatic yellow thermochromic crystals suitable for single-crystal X-ray diffraction (515 mg, 55\% yield based on ligand). We could not fully characterize this complex due to the pandemic shutdowns. 


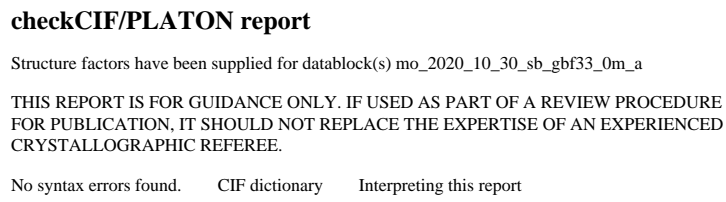

Figure 4.19: CheckCIF report for $\left(4 \mathrm{~Pb} \mathrm{~b}_{\mathrm{tPe}}\right)_{2}$. 


\subsubsection{5 (4Pb)(iPrNHC)}

Complex $(\mathbf{4 P b})_{2}(1 / 2$ eq., $0.693 \mathrm{~g}, 0.851 \mathrm{mmol})$ was dissolved in ca. $10 \mathrm{~mL}$ of hexane in a glass scintillation vial at room temperature to afford a bright orange solution. Then, a suspension of $N^{1}, N^{3}$-di-iso-propyl-4,5-dimethylimidazol-2-ylidene (1 eq., iPrNHC, $0.307 \mathrm{~g}$, $1.70 \mathrm{mmol})^{291}$ in ca. $10 \mathrm{~mL}$ hexane was added dropwise with stirring. Suspended iPrNHC immediately dissolved upon mixing with the solution of $(\mathbf{4 P b})_{2}$, and an immediate colour change to pale yellow was observed. Upon complete addition, the solution was stirred for $2 \mathrm{~h}$, after which the solution had slightly darkened and a precipitate formed. The mixture was filtered, then solvent and volatiles were removed under vacuum to leave a pale yellow fluffy solid (1.000 g, $1.70 \mathrm{mmol}$, quant.). ${ }^{1} \mathrm{H}$ NMR $\left(300 \mathrm{MHz}, \mathrm{C}_{6} \mathrm{D}_{6}, \delta_{\mathrm{C}_{6} \mathrm{D}_{5} H}=\right.$ $7.161 \mathrm{ppm}): 0.568\left(6 \mathrm{H}, s, \mathrm{Si}\left(\mathrm{CH}_{3}\right)_{2}\right), 1.24\left(12 \mathrm{H}, d, \mathrm{CN}-\mathrm{C}(\mathrm{H})\left(\mathrm{CH}_{3}\right)_{2},{ }^{3} J=29.4 \mathrm{~Hz}\right), 1.44(18 \mathrm{H}$, $\left.s, \mathrm{PbN}-\mathrm{C}\left(\mathrm{CH}_{3}\right)_{3}\right), 1.59\left(6 \mathrm{H}, s,=\mathrm{C}\left(\mathrm{CH}_{3}\right)\right), 5.37\left(2 \mathrm{H}\right.$, sept, $\left.\mathrm{CN}-\mathrm{C}(\mathrm{H})\left(\mathrm{CH}_{3}\right)_{2},{ }^{3} \mathrm{~J}=7.2 \mathrm{~Hz}\right)$. Attempts to recrystallize the solid from toluene at $-49{ }^{\circ} \mathrm{C}$ were frustrated by decomposition. A sample of the solid $(250 \mathrm{mg})$ darkened considerably upon attempted sublimation ( $\leq 90{ }^{\circ} \mathrm{C} / 10 \mathrm{mTorr}$ ), yielding non-stoichiometric mixtures of solid iPrNHC and $(\mathbf{4 P b})_{2}$ in the condenser. We could not fully characterize this complex due to the pandemic shutdowns.

\subsubsection{Lead(II) $N, N^{\prime}$-bis(1-(dimethylamino)-2-methylpropan-2-yl)-1,1- dimethylsilanediamide $(\mathrm{X})$}

Neat $\mathrm{Me}_{2} \mathrm{Si}\left(\mathrm{NH}^{t} \mathrm{Bu}\right)_{2}\left(4.048 \mathrm{~g}, 20.0 \mathrm{mmol}, 0.5\right.$ eq.) and neat $\mathrm{Me}_{2} \mathrm{SiCl}_{2}(2.581 \mathrm{~g}, 20.0 \mathrm{mmol}$, 0.5 eq.) were mixed for $80 \mathrm{~h}$ at room temperature $\left(\right.$ ca. $21^{\circ} \mathrm{C}$ ) in a $250 \mathrm{~mL}$ round-bottom flask. After this time, the mixture was diluted with $100 \mathrm{~mL}$ of hexane. Then, in a separate flask, MeLi (40.0 mmol, $25 \mathrm{~mL}$ of $1.6 \mathrm{M}$ solution in $\mathrm{Et}_{2} \mathrm{O}, 1$ eq.) was added dropwise by syringe to a stirred solution of $\mathrm{Me}_{2} \mathrm{NCH}_{2} \mathrm{C}\left(\mathrm{Me}_{2}\right) \mathrm{NH}_{2}(5.067 \mathrm{~g}, 40.0 \mathrm{mmol}, 1$ eq.) in ca. $50 \mathrm{~mL} \mathrm{Et}_{2} \mathrm{O}$ in a $200 \mathrm{~mL}$ Schlenk flask at room temperature over $30 \mathrm{~min}$. After $2 \mathrm{~h}$, a white precipitate had developed (presumably the lithium amide; not isolated), the ethereal suspension was transferred into a $125 \mathrm{~mL}$ pressure-equalized dropping funnel and added 
dropwise to the hexane solution of $\mathrm{Me}_{2} \mathrm{SiCl}\left(\mathrm{NH}^{t} \mathrm{Bu}\right)$ at room temperature over $30 \mathrm{~min}$ and stirred overnight (ca. 16h). The reaction mixture was then filtered to remove solid $\mathrm{LiCl}$, and volatiles were removed from the clear colourless filtrate under vacuum to yield a colourless oil and a suspended colourless solid. The mixture was taken up in pentane, filtered, and the volatiles removed again under vacuum to yield a colourless oil. This oil was then distilled by vacuum transfer to yield a similar colourless oil (6.755 g, 69\% assuming conversion to $\left.\mathrm{Me}_{2} \mathrm{Si}\left(\mathrm{NH}^{t} \mathrm{Bu}\right)\left[\mathrm{NH}\left(\mathrm{CMe}_{2} \mathrm{CH}_{2} \mathrm{NMe}_{2}\right)\right]\right)$. $1 \mathrm{H}$ NMR spectroscopy of the oil in $\mathrm{CDCl}_{3}$ suggested the oil mostly consisted of $\mathrm{Me}_{2} \mathrm{Si}\left(\mathrm{NH}^{t} \mathrm{Bu}\right)\left[\mathrm{NH}\left(\mathrm{CMe}_{2} \mathrm{CH}_{2} \mathrm{NMe}_{2}\right)\right]$, but in $\mathrm{C}_{6} \mathrm{D}_{6}$ there was a forest of peaks and the product could not be identified. We were unable to fully characterize this reaction and its products due to the pandemic shutdown. But, considering complex $\mathbf{X}$ was eventually isolated from a reaction using this unknown mixture, it was likely a mixture of $\mathrm{Me}_{2} \mathrm{Si}\left(\mathrm{NH}^{t} \mathrm{Bu}\right)\left[\mathrm{NH}\left(\mathrm{CMe}_{2} \mathrm{CH}_{2} \mathrm{NMe}_{2}\right)\right]$ and $\mathrm{Me}_{2} \mathrm{Si}\left[\mathrm{NH}\left(\mathrm{CMe}_{2} \mathrm{CH}_{2} \mathrm{NMe}_{2}\right)\right]_{2}$. ${ }^{1} \mathrm{H}$ NMR spectroscopy of the solid in $\mathrm{CDCl}_{3}$ was silent, so it was presumably $\mathrm{LiCl}$.) The oil was used for the next step without further purification.

Lead (II) bis[bis(trimethylsilyl)amine] (0Pb, $1.927 \mathrm{~g}, 3.65 \mathrm{mmol})$ and the unidentified oil (0.900 g, ca. $3.65 \mathrm{mmol}$ assuming pure $\left.\mathrm{Me}_{2} \mathrm{Si}\left(\mathrm{NH}^{t} \mathrm{Bu}\right)\left[\mathrm{NH}\left(\mathrm{CMe}_{2} \mathrm{CH}_{2} \mathrm{NMe}_{2}\right)\right]\right)$ were mixed together in $50 \mathrm{~mL}$ of toluene, then heated to $70{ }^{\circ} \mathrm{C}$ overnight (ca. $15 \mathrm{~h}$ ). After cooling to room temperature, the solution was a bright orange. Solvent was removed from an aliquot (ca. $2 \mathrm{~mL}$ ) under vacuum, the crude residue dissolved in $\mathrm{C}_{6} \mathrm{D}_{6}$, and analyzed by ${ }^{1} \mathrm{H}$ NMR spectroscopy. Starting material was observed, so the reaction mixture was heated to $70{ }^{\circ} \mathrm{C}$ overnight again (ca. $16 \mathrm{~h}$ ) to effect completion. The next day, metallic $\mathrm{Pb}(0)$ had precipitated and there was a deep red supernatant liquid above it. The mixture was filtered over Celite and a medium porosity glass frit, and the precipitate washed with three portions of ca. $5 \mathrm{~mL}$ toluene. Volatiles were removed under vacuum to leave behind a small amount of an orange pasty solid. Yellow thermochromic low-melting point crystals co-sublimed with a colourless liquid from this crude solid under vacuum $\left(70{ }^{\circ} \mathrm{C} / 40 \mathrm{mTorr}\right)$. This crude product was then dissolved in pentane and cooled to $-49{ }^{\circ} \mathrm{C}$ for 3 days, when 
large yellow crystals were deposited (ca. $150 \mathrm{mg}$ ). However, the solid was very soluble in a variety of solvents and would redissolve upon the slightest warming, so the pentane was removed under vacuum, and 3 drops of heptane were added. The solution was cooled to $-33{ }^{\circ} \mathrm{C}$ and yellow crystals had grown a week later. Crystals were mechanically removed from the heptane, placed on a microscope slide on a pre-cooled Al block $\left(-196{ }^{\circ} \mathrm{C}\right)$ and kept under a cold $\mathrm{N}_{2}$ stream delivered with a squirt bottle filled with liquid $\mathrm{N}_{2}$. This kept the crystals cold and air-free long enough for one to be selected and mounted. Single-crystal XRD then revealed the crystals were in fact lead(II) $N, N^{\prime}$-bis(1(dimethylamino)-2-methylpropan-2-yl)-1,1- dimethylsilanediamide (X). Half of the solution from before had been separated and recrystallized. The solvent was then removed to yield what was also presumably mostly $\mathbf{X}$ (ca. $80 \mathrm{mg}$, $5 \%$ yield based on $\mathbf{0 P b}$ ), which was then used for TGA. We were unable to fully characterize this reaction and its products due to the pandemic shutdown.

\subsubsection{Thermogravimetric Analysis}

Thermogravimetric analysis (TGA) was performed on Pt pans with a TA Instruments Q50 housed in an MBraun Labmaster 130 dry-box filled with nitrogen gas (99.998\% purity). Pt pans were cleaned by sequential ultrasonication in dilute nitric acid (ca. $3 \mathrm{~N}$ ), water, then 2-propanol. They were then heated until red hot by a propane torch flame in air to remove remaining impurities. Pt pans were then coated before each experiment with approximately $55 \mathrm{~nm}$ of $\mathrm{Al}_{2} \mathrm{O}_{3}$ by atomic layer deposition (ALD) in a Picosun R-150 reactor using 500 cycles of trimethylaluminum (TMA) and water at $200{ }^{\circ} \mathrm{C}$ with $0.1 \mathrm{~s}$ pulses and $8 \mathrm{~s}$ purges each. This is critical to protect the Pt pans as they become brittle and break easily after high-temperature exposure to $\mathrm{Pb}(0)$ residues from precursor decomposition. Ramp experiments were performed under a flow of ultrapure nitrogen ("5.0" 99.999\% purity, $60 \mathrm{sccm}$ ) at $10{ }^{\circ} \mathrm{C} \mathrm{min}^{-1}$ to a maximum temperature of $500{ }^{\circ} \mathrm{C}$. Stress-tests were performed under the same conditions sequentially on freshly cleaned pans with in- 


\section{checkCIF/PLATON report}

Structure factors have been supplied for datablock(s) mo_2020_11_13_jdm_sb_gbsuperso_a

THIS REPORT IS FOR GUIDANCE ONLY. IF USED AS PART OF A REVIEW PROCEDURE FOR PUBLICATION, IT SHOULD NOT REPLACE THE EXPERTISE OF AN EXPERIENCED CRYSTALLOGRAPHIC REFEREE

No syntax errors found. CIF dictionary Interpreting this report

Datablock: mo_2020_11_13_jdm_sb_gbsuperso_a

\begin{tabular}{|c|c|c|c|}
\hline Bond precision: & $\mathrm{C}-\mathrm{C}=0.0037 \mathrm{~A}$ & Wavelength & $h=0.71073$ \\
\hline Cell: & $\begin{array}{l}a=10.5894(3) \\
\text { alpha }=90\end{array}$ & $\begin{array}{l}\mathrm{b}=15.4015(4) \\
\mathrm{bet} a=97.949(1)\end{array}$ & $\begin{array}{l}\mathrm{C}=12.0598(3) \\
\text { gamma }=90\end{array}$ \\
\hline \multirow[t]{2}{*}{ Temperature: } & $125 \mathrm{~K}$ & & \\
\hline & Calculated & Reported & \\
\hline Volume & $1947.97(9)$ & $1947.97(9)$ & \\
\hline Space group & C $2 / c$ & C $12 / c_{1}$ & \\
\hline Hall group & $-\mathrm{C} 2 \mathrm{yc}$ & $-\mathrm{C} 2 \mathrm{yc}$ & \\
\hline Moiety formula & $\mathrm{C} 14 \mathrm{H}_{34} \mathrm{~N} 4 \mathrm{~Pb} \mathrm{Si}$ & C14 H34 & $\mathrm{N} 4 \mathrm{~Pb} \mathrm{Si}$ \\
\hline $\begin{array}{l}\text { Sum formula } \\
\text { Mr }\end{array}$ & $\begin{array}{l}\mathrm{C} 14 \quad \mathrm{H} 34 \mathrm{~N} 4 \mathrm{~Pb} \mathrm{Si} \\
493.74\end{array}$ & $\begin{array}{l}\text { C14 H34 } \\
493.73\end{array}$ & $\mathrm{~N} 4 \mathrm{~Pb} \mathrm{Si}$ \\
\hline $\mathrm{Dx}, \mathrm{g} \mathrm{cm}-3$ & 1.684 & 1.684 & \\
\hline $\mathrm{z}$ & 4 & 4 & \\
\hline $\mathrm{Mu}(\mathrm{mm}-1)$ & 8.721 & 8.721 & \\
\hline F000 & 968.0 & 968.0 & \\
\hline F000' & 955.55 & & \\
\hline$h, k, I \max$ & $13,20,15$ & $13,20,15$ & \\
\hline Nref & 2357 & 2356 & \\
\hline$T \min , T \max$ & $0.084,0.208$ & $0.294,0.7$ & 747 \\
\hline Tmin' & 0.053 & & \\
\hline \multicolumn{2}{|c|}{$\begin{array}{l}\text { Correction method= \# Reported } \mathrm{T} \\
\text { AbsCorr = MULTI-SCAN }\end{array}$} & Limits: $\operatorname{Tmin}=0.294$ & $\operatorname{Tmax}=0.747$ \\
\hline \multicolumn{2}{|c|}{ Data completeness $=1.000$} & Thet $a(\max )=27.99$ & \\
\hline$R($ reflections $)=$ & $0.0200(2319)$ & WR2 (reflections) = & $=0.0504(2356)$ \\
\hline$S=1.183$ & Npar $=$ & 97 & \\
\hline
\end{tabular}

The following ALERTS were generated. Each ALERT has the format

test-name_ALERT_alert-type_alert-1evel.
click on the hyperlinks for more details of the test.

Figure 4.20: CheckCIF report for $X$. 
crementally increasing initial mass loadings. Vapour pressures were estimated using a literature method. ${ }^{102,103}$ Vaporization constants $\alpha \mathrm{m}$ (from the Langmuir equation) were obtained for our instrument by calibrating compounds with known Antoine equation parameters and volatilities similar to the analyte. Benzoic acid $\left(\alpha_{\mathrm{TGA}}=2.016 \times 10^{-4}\right)$ was used as a calibrant for all compounds. These data were used to find the pressure $p$ as a function of $T$, thus $\ln \mathrm{p}$ as a function of $T^{-1}$, which can be modelled with the Clausius-Clapeyron equation. The slope of this line is equal to the enthalpy of sublimation (before melting) and evaporation (after melting) divided by the gas constant $\Delta H / R$.

\subsubsection{Computational Details}

Quantum chemical calculations were entirely performed with ORCA v4.2.1. ${ }^{237}$ Geometries were optimized with the revised Perdew-Burke-Ernzerhof functional of Zhang and Yang (revPBE) ${ }^{292}$ including Grimme’s atom-pairwise dispersion correction with Becke-Johnson damping [D3(BJ)]. ${ }^{239,240}$ Analytical Hessian calculations were performed to confirm there were no imaginary vibrational modes and the geometries were minima on the potential energy surface. Scalar-relativistic effects were accounted for with the zero-order regular approximation (ZORA). ${ }^{293-295}$ The exponents from Ahlrich's double- $\zeta$ split-valence quality basis set $^{241}$ recontracted for ZORA (ZORA-def2-SVP) was used on all atoms except $\mathrm{Pb}$, which used the triple- $\zeta$ quality segmented all-electron relativistically contracted basis set (SARC-ZORA-TZVP) of Pantazis and Neese. ${ }^{296}$ Here we denote the basis set used on all other atoms outside parentheses and the basis set used on $\mathrm{Pb}$ inside parentheses [e.g., ZORA-def2-SVP(SARC-ZORA-TZVP)]. The auxiliary basis sets def2/J of Weigend $(\mathrm{H}-\mathrm{Kr}),{ }^{244}$ and SARC/J of Pantazis and Neese $(\mathrm{Pb})^{296}$ was used in all calculations. Inclusion of relativistic effects was critical to obtain geometries of the dimers that matched experiment. The standard pseudopotentials for $\mathrm{Pb}$ were found to be inadequate, but the use of all-electron basis sets had a marginal effect on the cost of all calculations. A benchmark optimization using the hybrid functional of Zhao and Truhlar [PW6B95] ${ }^{297}$ showed neg- 
ligible improvement over revPBE for $(\mathbf{4 P b})_{2}$ and was not worth the substantially higher cost. For single-point energy and property calculations, the quadruple- $\zeta$ with polarization basis set (ZORA-def2-QZVPP) was used on all atoms except $\mathrm{Pb}$, which used the triple- $\zeta$

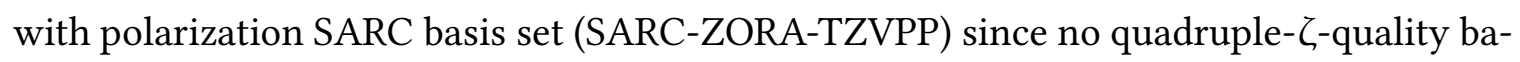
sis set for $\mathrm{Pb}$ has yet been made available. Molecular electrostatic potentials (MEP) were obtained using densities from Head-Gordon's range-separated hybrid functional modified by Najibi and Goerigk ${ }^{238}$ to include Grimme’s dispersion correction with Becke-Johnson damping ${ }^{239,240}$ using the large basis sets on the previously optimized geometries [ $\omega$ B97XD3(BJ)/ZORA-def2-QZVPP(SARC-ZORA-TZVPP)//revPBE/ZORA-def2-SVP(SARC-ZORATZVP)]. Resolution-of-the-identity approximation for the Coulomb integrals with the chainof-spheres approximation for the exchange term (RIJCOSX) ${ }^{243}$ was used to speed up the self-consistent field (SCF) step of the hybrid DFT calculations. Single-point calculations using the domain-based local pair natural orbital coupled cluster with singles, doubles, and perturbative triples of Riplinger et al. ${ }^{298}$ were performed using DFT geometries and DFT reference wavefunctions using the large basis sets [DLPNO-CCSD(T)/ZORA-def2QZVPP (SARC-ZORA-TZVPP)//revPBE/ZORA-def2-SVP(SARC-ZORA-TZVP)]. NormalPNO convergence criteria were used due to the large size of the systems considered, and the increased cost and accuracy with TightPNO criteria could not be justified without more experimental results to compare with. Wavefunction analysis was performed with Multiwfn v3. $7^{299}$ running on a personal computer. Analysis performed and included here: Mayer bond order (MBO), ${ }^{300}$ Bader's quantum topology of atoms-in-molecules (QTAIM), ${ }^{301,302}$ Johnson's non-covalent interaction (NCI) analysis, ${ }^{276}$ and Murray and Politzer's MEP surface analysis. ${ }^{286}$ All structures, energies, orbitals, and wavefunctions are available upon request. 


\section{Chapter 5}

\section{A Volatile and Thermally Stable Co(IV) Bis $(\beta$-silyldiamide)}

Reprinted and adapted from the original communication published as:

David Zanders, ${ }^{c, b}$ Goran Bačić, ${ }^{c}$ Dominique Leckie, ${ }^{w}$ Oluwadamilola Odegbesan,,${ }^{c}$ Jeremy Rawson, ${ }^{w}$ Jason D. Masuda, ${ }^{m}$ Anjana Devi, ${ }^{b}$ and Seán T. Barry. ${ }^{c}$ A Rare Low-Spin Co ${ }^{\mathrm{IV}}$ Bis( $\beta$-silyldiamide) with High Thermal Stability: Steric Enforcement of a Doublet Configuration. Angew. Chem. Int. Ed., 2020, 59, 14138-14142.

c Department of Chemistry, Carleton University, 1125 Colonel By Drive, Ottawa, Ontario, K1S 5B6, Canada

$b$ Inorganic Materials Chemistry, Faculty of Chemistry and Biochemistry, Ruhr University Bochum, Universitätsstraße 150, 44801, Bochum, Germany

$w$ Department of Chemistry and Biochemistry, University of Windsor, 401 Sunset Avenue, Windsor, Ontario, N9B 3P4, Canada

$m$ Department of Chemistry, Saint Mary's University, 923 Robie Street, Halifax, Nova Scotia, Canada, B3H 3C3 


\section{Abstract}

Attempted preparation of a chelated $\mathrm{Co}(\mathrm{II}) \beta$-silylamide resulted in the unprecedented disproportionation to $\mathrm{Co}(0)$ and a spirocyclic cobalt(IV) bis $\left(\beta\right.$-silyldiamide): $\left[\mathrm{Co}\left[\left(\mathrm{N}^{t} \mathrm{Bu}\right)_{2} \mathrm{SiMe}_{2}\right]_{2}\right]$ (4Co). Compound 4Co exhibited a room temperature magnetic moment of 1.8 B.M. and a solid state axial EPR spectrum diagnostic of a rare $S=1 / 2$ configuration. Ab initio semicanonical coupled-cluster calculations (DLPNO-CCSD $(\mathrm{T})$ ) revealed the doublet state was clearly preferred $(-27 \mathrm{kcal} / \mathrm{mol})$ over higher spin configurations only for the bulky tertbutyl substituted analogue. Unlike other Co(IV) complexes, 4Co had remarkable thermal stability, and was demonstrated to form a stable self-limiting monolayer in preliminary atomic layer deposition (ALD) surface saturation experiments. The ease of synthesis and high stability make $\mathbf{4 C o}$ an attractive starting point to investigate otherwise inaccessible $\mathrm{Co}(\mathrm{IV})$ intermediates and for synthesizing new materials. 


\subsection{Introduction}

Cobalt complexes are effective and cost-efficient alternatives to Ir- and Rh-based catalysts for a number of important $\mathrm{C}-\mathrm{H}$ bond transformations, ${ }^{303,304}$ alkynelations, ${ }^{305,306}$ alkylations, and arylations. ${ }^{307,308}$ Recently, high-valent Co(IV) species have also been shown to effect $\mathrm{C}-\mathrm{H}$ bond activation. ${ }^{309}$ High-valence Co-containing materials like the electrode material $\mathrm{Li}_{2-x} \mathrm{CoO}_{2-\delta}$ are also essential as the cathode material of Li-ion batteries, ${ }^{310,311}$ and others such as $\operatorname{CoS}_{x}$ have been predicted to become key materials in future green energy photocatalysts. ${ }^{312-315}$ The facile synthesis of robust Co(IV) complexes has remained a challenge, so their exploitation in the field of catalysis as active species and in materials science as precursors for material synthesis has remained limited. Chemical vapour phase (CVD) techniques such as atomic layer deposition (ALD) have become one of the most preferable approaches for the fabrication of exotic, emerging materials. ${ }^{42,316}$ So it stands to reason to evaluate $\mathrm{Co}(\mathrm{IV})$ complexes not only in light of their electronic nature but also with respect to their physicochemical properties which might qualify them as CVD/ALD precursors.

To date, most $\mathrm{Co}(\mathrm{IV})$ complexes have been prepared by $1 e^{-}$oxidation of $\mathrm{Co}(\mathrm{III})$ complexes with $\mathrm{Br}_{2}, \mathrm{Fe}\left(\mathrm{ClO}_{4}\right)_{3}$ or by cyclic voltammetry. The starting $\mathrm{Co}(\mathrm{III})$ complexes typically comprise sterically demanding tetraamido or pyrrolato macrocycles, and the resulting Co(IV) species are commonly unstable above room temperature. In cases where unambiguous $\mathrm{Co}(\mathrm{IV})$ species could be isolated, geometries are typically limited to square planar and square pyramidal. ${ }^{317-319}$ Macrocyclic Co(IV) oxo-congeners such as the one introduced by Wang et al. are typically more robust but still suffer from this geometrical restriction. ${ }^{287}$

For $\mathrm{d}^{5}$ ions, the high-spin $S=5 / 2$ configuration is stabilized by a maximized exchange energy, thus examples of low-spin $\mathrm{d}^{5}$ complexes are rare. However, for Co(IV), the increased oxidation state affords stronger ligand fields and so a finer energetic bal- 
ance is expected between high- and low-spin electronic configurations. Interesting exam-

ples are Deng's trigonal planar, N-heterocyclic carbene stabilized Co(IV) bis-imide ${ }^{320}$ and Groysman's tri-coordinate, diarylcarbene stabilized Co(IV) bis-alkoxide. ${ }^{309}$ While computational studies showed that the assignment of the spectroscopic oxidation state for the metal centers was ambiguous, their magnetic properties supported a $S=1 / 2$ ground state in each case. More recently, a squashed-tetrahedral $\mathrm{Co}(\mathrm{IV})$ tetrakis(ketimide) $\mathrm{Co}\left(\mathrm{N}=\mathrm{C}^{t} \mathrm{Bu}_{2}\right)_{4}$ was reported, but it exhibited a quartet ground state $(S=3 / 2) .{ }^{321} \operatorname{Co}(\mathrm{IV})$ tetrakis $(1-$ norbornyl) $\mathrm{Co}(\text { nor })_{4}$ has remained the only known unambiguous example of a tetracoordinate low-spin Co(IV) complex for over 40 years. ${ }^{322,323}$

Bulky $\beta$-silylamines are excellent ligands that stabilize unusual coordination numbers and oxidation states for elements across the periodic table. ${ }^{324,325}$ The good thermal stability and high volatility of the well-known three-coordinate $\mathrm{Co}(\mathrm{II})$ complex $\left\{\mathrm{Co}\left[\mathrm{N}\left(\mathrm{SiMe}_{3}\right)_{2}\right]_{2}\right\}_{2}$ prompted us to explore other Co $\beta$-silylamides as potential precursors for vapour phase deposition of Co containing materials. Here, we report the serendipitous preparation of 1,3,5,7-tetra-tert-butyl-2,2,6,6-tetramethyl-1,3,5,7-tetraaza-2,6-disila-4 $\lambda^{4}$-cobaltaspiro-[3.3] heptane $\left(\mathrm{Co}\left[\left(\mathrm{N}^{t} \mathrm{Bu}\right)_{2} \mathrm{SiMe}_{2}\right]_{2}, 4 \mathrm{Co}\right)$, the second unambiguously $\mathrm{Co}(\mathrm{IV})$ complex with a low-spin $S=1 / 2$ ground state configuration. We used experiment and theory to understand its unusual electronic nature. Finally, compound 4Co has electronic properties that are complemented by physico-chemical features that set it apart from all prior reported Co(IV) complexes and make it especially appealing for application in ALD.

\subsection{Results and Discussion}

\subsubsection{Co(IV) Bis[bis(tert-butylamido)dimethylsilane]}

Treatment of suspended $\mathrm{CoCl}_{2}$ (tmeda) with one equivalent of $(\mathrm{LiNtBu})_{2} \mathrm{SiMe}_{2}$ in pentane led to a rapid change in color from ultramarine to dark brown-black. Dark grey particles were observed to precipitate from solution alongside $\mathrm{LiCl}$ following the disproportiona- 
tion of the presumed Co(II) monokis(diamide) intermediate to form $\mathbf{4 C o}$ and elemental $\mathrm{Co}(0)$. Workup of the reaction solution afforded 4Co as a dark-brown, almost black crystalline solid that was purified either by recrystallization from pentane at $-49^{\circ} \mathrm{C}$ or vacuum sublimation $\left(85^{\circ} \mathrm{C} / 10 \mathrm{mTorr}\right)$. The synthesis scaled well and satisfactory yields ( $60 \%$ from the ligand) were obtained from $10 \mathrm{mmol}$ batches with ease.
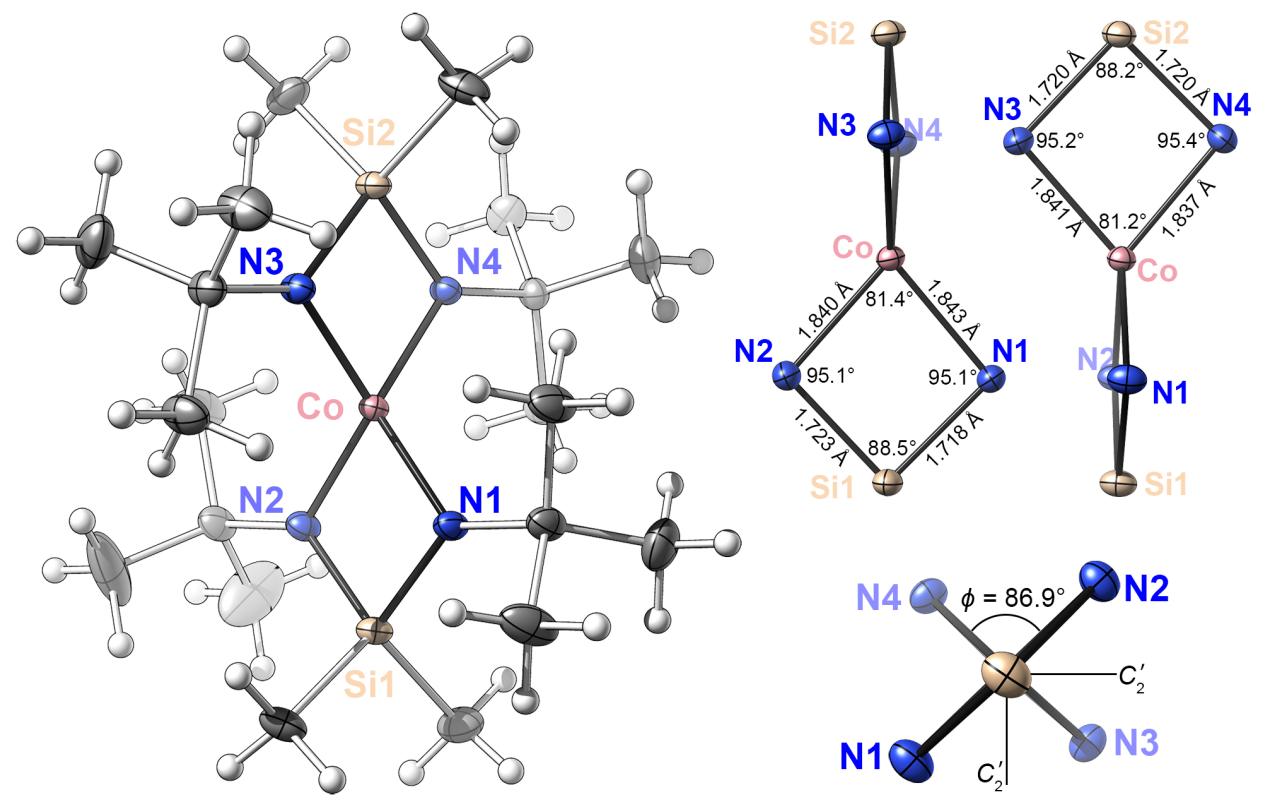

Figure 5.1: Solid state structure of 4Co (left), key bond lengths and angles of the two rings with the principal axis oriented vertically (top right) and ligand plane angle $\phi$ and the $C_{2}^{\prime}$ axes when looking down the principal axis (bottom right).

Single-crystal X-ray crystallography revealed $\mathbf{4 C o}$ was a four-coordinate inorganic spirocycle of $D_{2 d}$ symmetry (Figure 5.1). With a Yang geometry index value of $\tau_{4}=0.75$ (Figure 5.2), ${ }^{326}$ it lies between the two other known four-coordinate homoleptic Co(IV) complexes: Hayton's $\mathrm{Co}\left(\mathrm{N}=\mathrm{C}^{t} \mathrm{Bu}_{2}\right)_{4}\left(\tau_{4}=0.59\right)^{321}$ and $\mathrm{Co}(\text { nor })_{4}\left(\tau_{4}=0.94\right) .{ }^{323}$ The average $\mathrm{Co}-\mathrm{N}$ bond length of $1.84 \AA$ was only slightly longer than in $\mathrm{Co}\left(\mathrm{N}=\mathrm{C}^{t} \mathrm{Bu}_{2}\right)_{4}$ $(1.80 \AA)$, and notably shorter than the $\mathrm{Co}-\mathrm{C}$ bond in $\mathrm{Co}(\text { nor })_{4}(1.92 \AA)$. Coordination around each nitrogen was planar, typical of silylamides due to electrostatic repulsion of the polarized $\mathrm{Si}-\mathrm{N}$ bonds. The ligand planes were arranged almost orthogonally in $4 \mathrm{Co}$ $\left(\phi=86.9^{\circ}\right)$, contrasting Hayton's complex which was twisted toward $D_{4 h}$ symmetry $(\phi=$ 
$\left.57.1^{\circ}\right)$. Both shared the same $\mathrm{Co}-\mathrm{N}$ coordination sphere, but the increased steric bulk and geometric rigidity of the $\beta$-silyldiamide locked the ligands on $\mathbf{4 C o}$ into an orthogonal arrangement. This being the case, we expected significant differences in the electronic structure of $4 \mathrm{Co}$ and $\mathrm{Co}\left(\mathrm{N}=\mathrm{C}^{t} \mathrm{Bu}_{2}\right)_{4}$.

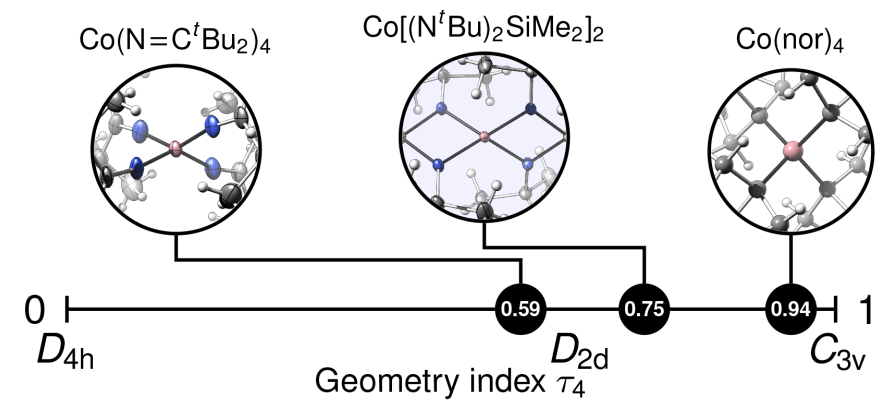

Figure 5.2: Yang's geometry index $\tau_{4}$ for known tetracoordinate $\mathrm{Co}(\mathrm{IV})$ complexes.

Room temperature magnetic susceptibility measurements of 4Co (Gouy method) revealed an effective magnetic moment of $\mu_{\mathrm{eff}}=(1.8 \pm 0.1) \mathrm{BM}$ in the solid state, suggesting 4Co adopts an unusual low-spin doublet $(S=1 / 2)$ ground state configuration. This was supported by solid state EPR spectra which displayed axial spectra consistent with a doublet state $\left(g_{z}=2.6, g_{x}=g_{y}=2.0\right.$, Figure 5.3). Variable-temperature EPR studies showed no significant dependence on temperature from $125-300 \mathrm{~K}$. The $g$-tensor is similar to that of a low-spin four-coordinate $\mathrm{d}^{5} \mathrm{Fe}(\mathrm{III})$ complex reported by Peters which adopted $C_{3 v}$ symmetry $\left(g_{z}=2.9, g_{x}=g_{y}=2.0\right) .{ }^{327}$ The doublet ground state was also supported by density functional theory (DFT) calculations, as we calculated $g_{z}=2.4, g_{x}=g_{y}=2.0$ for the doublet state and $g_{z}=g_{x}=g_{y}=2.0$ for the quartet and sextet states.

\subsubsection{Steric Control of the Electronic Structure}

We turned to DFT and ab initio coupled-cluster theory to understand the electronic structure of 4Co. Accurate prediction of spin-states with DFT is an ongoing challenge because the exact exchange-correlation functional is still unknown. And while mixing a portion of exact Hartree-Fock (HF) exchange into hybrid functionals generally improves perfor- 

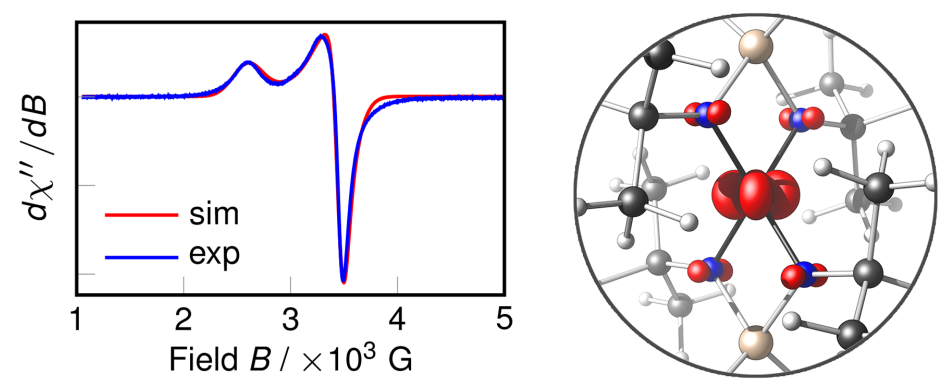

Figure 5.3: (Left) Experimental (blue, front) and simulated (red, back) solid state X-band EPR spectra of 4Co. (Right) Spin density of 4Co (def2-QZVPP//OPBE-D3(BJ)/def2-SVP, iso-surface value $=0.02$ ), highlighting the metal-based character of the unpaired electron.

mance for closed-shell molecules, these methods are biased toward high-spin transition metal configurations. ${ }^{328}$ Nevertheless, all of the pure functionals (BLYP, OLYP, PBE, OPBE) and one hybrid functional (PW6B95) accurately reproduced the experimental structure of 4Co when a doublet ground state was considered. The quartet and sextet spin-states did not: the quartet deviated by exhibiting a smaller ligand interplane angle $\left(\phi=72^{\circ}\right)$, and the sextet had elongated Co-N bonds (ca. $1.94 \AA$ ). General agreement between functionals on the geometries of the different spin-states suggested the structures were reasonable, but not necessarily their relative energies. Thus we proceeded with geometries from PW6B95-D3(BJ) DFT calculations to high-level ab initio methods for more accurate energies.

Unambiguous assignment of the doublet ground state to $4 \mathrm{Co}$ was achieved with ab initio semi-canonical coupled-cluster calculations of our best DFT-optimized structures (DLPNO-CCSD(T)/def2-TZVPP//PW6B95-D3(BJ)/def2-SVP). This recent method provides comparable performance to canonical $\operatorname{CCSD}(\mathrm{T})$, the "gold standard" of quantum chemistry, but with computational cost comparable to DFT. ${ }^{298}$ The doublet was calculated to be $28.1 \mathrm{kcal} / \mathrm{mol}$ and $27.1 \mathrm{kcal} / \mathrm{mol}$ more stable than the quartet or sextet, respectively. Our prior DFT calculations had substantial errors of $+22 \mathrm{kcal} / \mathrm{mol}$ and $+24 \mathrm{kcal} / \mathrm{mol}$ for the pure and hybrid functionals, respectively, compared to DLPNO-CCSD(T). We hope that these benchmarked results of a difficult case may act as reference values in the fu- 
ture.

Only three Co(IV) complexes with unambiguous doublet ground states have been reported before: $\mathrm{Co}(\text { nor })_{4}$, Deng's $\mathrm{Co}(=\mathrm{Ndipp})_{2}(\mathrm{NHC})$, and Groysman's $\left.\mathrm{Co}(\mathrm{OR})_{2}\left(=\mathrm{CPh}_{2}\right)\right)$. But their apparent oxidation states are their only similarity to 4Co. Each belongs to a different symmetry point group ( $T_{d}$ and $C_{2 v}$ respectively); their bonds are distinct from the Co $-\mathrm{N}$ bonds in $1 ; \mathrm{Co}(=\mathrm{Ndipp})_{2}(\mathrm{NHC})$ and $\left.\mathrm{Co}(\mathrm{OR})_{2}\left(=\mathrm{CPh}_{2}\right)\right)$ are heteroleptic and three coordinate; and the oxidation states in $\mathrm{Co}(=\mathrm{Ndipp})_{2}(\mathrm{NHC})$ and $\left.\mathrm{Co}(\mathrm{OR})_{2}\left(=\mathrm{CPh}_{2}\right)\right)$ were tentatively assigned alongside possible Co(III) resonant forms for which no precedence was found in $\mathbf{4 C o}$. Collectively, these dissimilarities made them less useful in explaining the doublet state of $\mathbf{4 C o}$. So, despite their different ground states, consideration of Hayton's $\mathrm{Co}\left(\mathrm{N}=\mathrm{C}^{t} \mathrm{Bu}_{2}\right)_{4}$ was instructive as it shared the same $\mathrm{Co}-\mathrm{N}$ coordination sphere as 4Co.

We constructed a qualitative molecular orbital diagram of the doublet and quartet configurations of 4Co using DFT (Figure 4). Although both states had molecular $D_{2 d}$ symmetry, the molecular orbitals were mostly of lower symmetry in the $D_{2}$ point subgroup. Exceptions to this were observed for unpaired electrons: the doublet SOMO retained full $D_{2 d}$ symmetry, but the quartet SOMOs were of even lower $C_{1}$ symmetry. The doublet's MOs were predictable from its symmetry, with the orthogonal ligand orientation stabilizing $\sigma$ and $\pi$-bonds involving the $b_{2}\left(\mathrm{~d}_{x z}\right)$ and $b_{3}\left(\mathrm{~d}_{y z}\right)$, and $b_{1}\left(\mathrm{~d}_{x^{2}-y^{2}}\right)$ DOMOs, respectively. The corresponding $\pi^{*}$-antibonding $b_{1}\left(\mathrm{~d}_{x^{2}-y^{2}}\right)$ SOMO showed $\pi$-backbonding in doublet $4 \mathbf{C o}$, which was also observed by Mulliken spin-population analysis (0.52e on Co and $0.52 e$ across four nitrogens). Despite their differences, we found an important parallel between the quartet state of $\mathbf{4 C o}$ and Hayton's $\mathrm{Co}\left(\mathrm{N}=\mathrm{C}^{t} \mathrm{Bu}_{2}\right)_{4}$. The twisted geometry and quartet ground state of $\mathrm{Co}\left(\mathrm{N}=\mathrm{C}^{t} \mathrm{Bu}_{2}\right)_{4}$ was due to $\pi$-backbonding of the unpaired electrons to the $\mathrm{N}=\mathrm{C} \pi$-bonds on the ligands, which was maximized with $D_{2 d}$ symmetry and $S=3 / 2 .{ }^{321}$ Clear $\pi$-backbonding to the $\mathrm{N}$ p-orbital was observed in the three highly distorted SOMOs of quartet $4 \mathrm{Co}$, with more than one unpaired electron residing on the nitrogens $(1.79 e$ on 


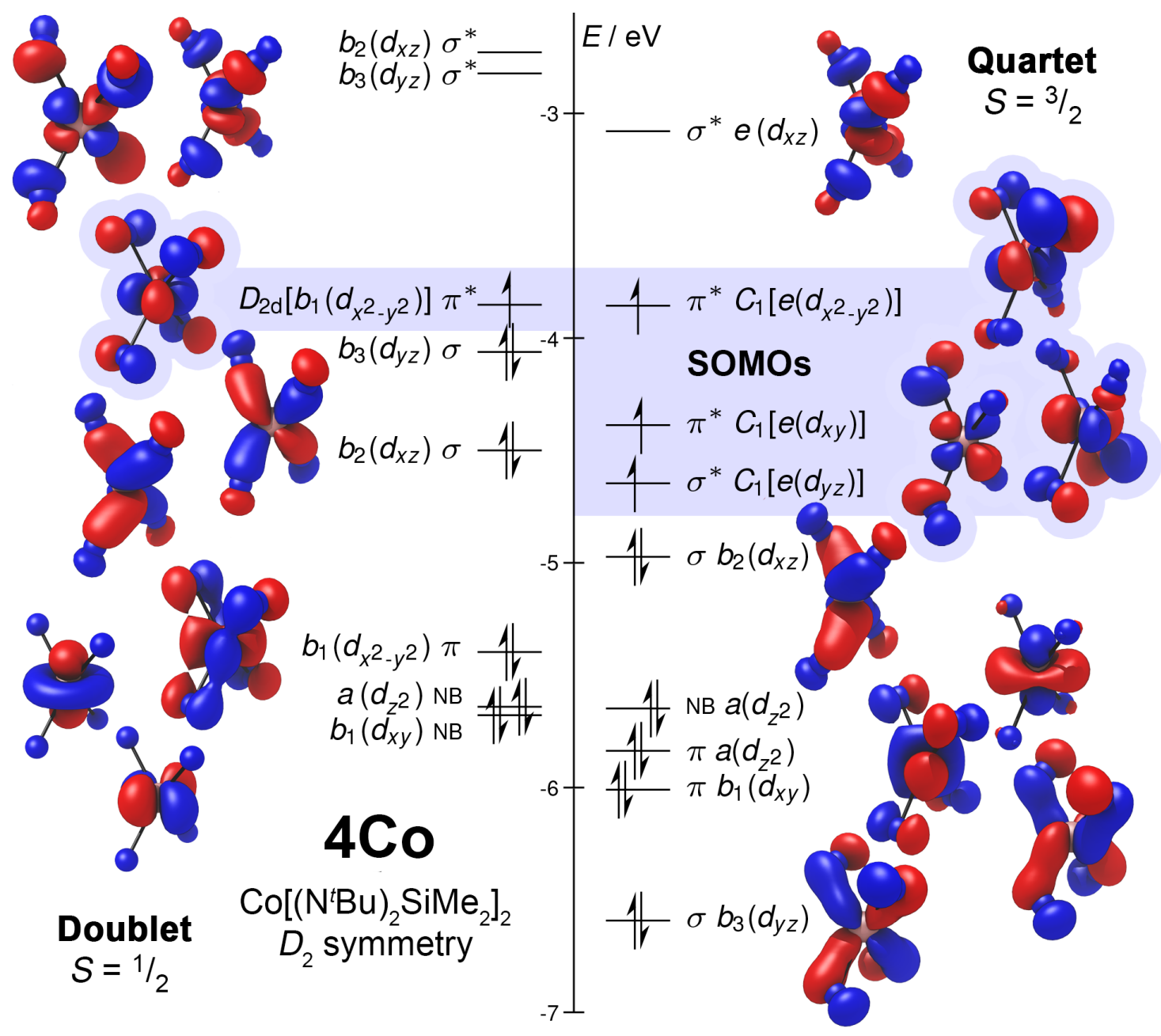

Figure 5.4: Unrestricted natural orbital (UNO) diagram of $\mathbf{4 C o}$ in the doublet (left) and quartet (right) spin states obtained with DFT (def2-QZVPP//OPBE-D3(BJ)def2-SVP). DOMOs and LUMOs have $D_{2}$ symmetry, while SOMO point groups are explicitly listed. Irreducible representations, participating Co d-orbitals, and bond type are listed for each. Hydrogen, carbon, and silicon have been omitted for clarity. 
Co and 1.21e across four nitrogens). Unfortunately, we could not reliably deconvolute the energetic contributions of these $\pi$-systems by energy decomposition analysis (EDA) because of the poor performance of DFT for $4 \mathrm{Co}$. Steric bulk clearly played an important role in stabilizing the doublet ground state of $\mathbf{4 C o}$, so we optimized the geometries and computed the doublet-quartet energy gaps for less bulky $\mathrm{N}$-substituents in the same way as for $\mathbf{4 C o}$ to quantify its effect (Figure 5).

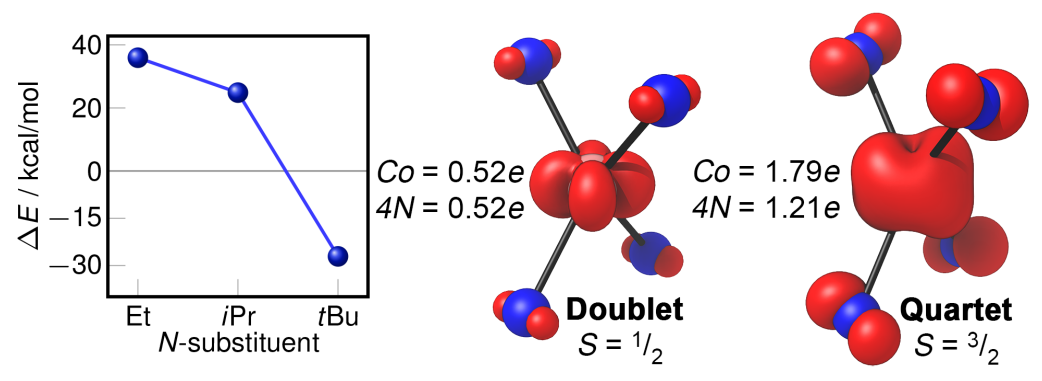

Figure 5.5: Doublet-quartet energy gaps [DLPNO-CCSD(T)/def2-TZVPP//PW6B95-D3(BJ)/def2SVP] for different N-substituted analogues of 4Co (left). Spin density (isosurface at $0.02 \mathrm{a} \mathrm{u}$ ) and Mulliken spin populations of 4Co in the doublet (middle) and quartet (right) ground states (def2QZVPP//OPBE-D3(BJ)/def2-SVP). Hydrogen, carbon, and silicon have been omitted for clarity.

All the successfully optimized doublet and quartet geometries had features consistent with those in $\mathbf{4 C o}$, and their DFT energy gaps also did not agree with the ab initio results. We found that reducing the bulk from just tert-butyl to iso-propyl made the quartet the most stable electronic configuration by a large margin $(\Delta E=+25 \mathrm{kcal} / \mathrm{mol})$. The SOMOs and spin populations of the quartet states were essentially independent of steric bulk, suggesting that the ligand plane angle $\phi$ was dependent on the $\mathrm{N}$-substituent more than on the extent of $\pi$-backbonding. Additionally, the geometric effect of the $\pi$-backbonding was clearly visible in the spin density plots of $\mathbf{4 C o}$. These results together demonstrate how the tert-butyl substituent's bulk was necessary to destabilize the otherwise preferred quartet ground state and it enforced the doublet ground state by orthogonal ligand arrangement. Analogues of $\mathbf{4 C o}$ with less steric bulk are expected to have electronic structures similar to $\mathrm{Co}\left(\mathrm{N}=\mathrm{C}^{t} \mathrm{Bu}_{2}\right)_{4}$. 


\subsubsection{Evaporation Kinetics and Self-Limiting Behaviour}

We tested the robustness of $\mathbf{4 C o}$ to examine its suitability for vapour phase deposition. As prior introduced, ALD is especially good at depositing metastable phases and exotic materials because of its self-limiting chemical mechanisms and mild operating conditions. ${ }^{329} \mathrm{To}$ our knowledge, there have been no reports of neither ALD nor CVD of Co(IV)-containing materials because of the lack of Co(IV) precursors. None of the few previously reported $\mathrm{Co}(\mathrm{IV})$ complexes displayed sufficient thermal stability or volatility. Co(nor $)_{4}$ decomposes at $100{ }^{\circ} \mathrm{C}$ and cannot be sublimed, Hayton's $\mathrm{Co}\left(\mathrm{N}=\mathrm{C}^{t} \mathrm{Bu}_{2}\right)_{4}$ decomposes in solution at ambient temperature, Deng's $\mathrm{Co}(=\mathrm{Ndipp})_{2}(\mathrm{NHC})$ undergoes reductive $\mathrm{C}-\mathrm{H}$ bond activation at $50{ }^{\circ} \mathrm{C}$, while Groysman's $\left.\mathrm{Co}(\mathrm{OR})_{2}\left(=\mathrm{CPh}_{2}\right)\right)$ is likely nonvolatile due to its high molecular weight and numerous phenyl groups.

We quantitatively evaluated the suitability of $\mathbf{4 C o}$ as a precursor with respect to its thermal stability and volatility by thermogravimetric analysis (TGA) and differential thermal calorimetry (DSC). When a $10.1 \mathrm{mg}$ sample was heated with a linearly increasing temperature ramp, a clean single-step mass loss was observed between $150-230{ }^{\circ} \mathrm{C}$ leaving behind a small residual mass of $3.2 \%$, indicating that most of the sample evaporated during the experiment (Figure 5.6a). TGA can also be used to efficiently estimate vapour pressure by employing the Langmuir equation, and the temperature at which $\mathbf{4 C o}$ displays 1 Torr of vapour pressure was estimated with a Clausius-Clapeyron model to be $(150.4 \pm 0.1){ }^{\circ} \mathrm{C}$ (Figure 5.6b). ${ }^{103}$ DSC revealed the melting point to be $143{ }^{\circ} \mathrm{C}$ and the onset of decomposition (defined as $5 \%$ of the maximum of the first exothermic event) ${ }^{81}$ to be $197{ }^{\circ} \mathrm{C}$. The "thermal range" between the 1 Torr vapour pressure temperature and the decomposition temperature is often a practical and reliable benchmark of a compound's practicality as an ALD precursor. With a thermal range spanning roughly $47^{\circ} \mathrm{C}$, compound $4 \mathrm{Co}$ represents a promising precursor candidate for ALD.

To contextualize the performance of $\mathbf{4 C o}$, we subjected two recently reported $\mathrm{Co}$ (II) 

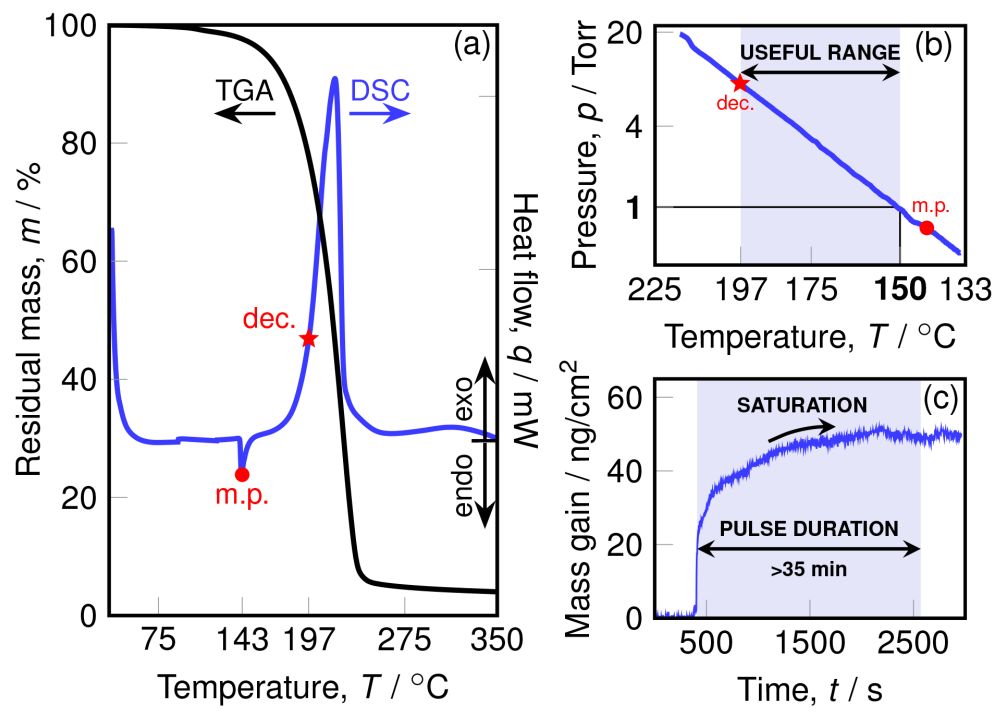

Figure 5.6: (a) TGA of a $10.1 \mathrm{mg}$ sample (black) and DSC (blue) of $0.3 \mathrm{mg}$ of 4 Co. (b) vapour pressure of $4 \mathrm{Co}$ and its useful range between the 1 Torr and decomposition temperatures. (c) Exposure of an alumina-coated quartz crystal microbalance (QCM) to 4Co demonstrating selflimited adsorption by the saturated mass gain $\left(\mathrm{ng} \mathrm{cm}^{-2}\right)$. Experimental details are provided in the SI for clarity.

precursors to thermal analysis: namely $\mathrm{CoCl}_{2}(\text { tmeda })^{330}$ and $\mathrm{Co}(\mathrm{dad})_{2}{ }^{331}(\mathrm{dad}=$ di-tertbutyl-1,2-diazabuta-1,2-diene) (see SI). We applied our recently developed "figure of merit" $\sigma$ that takes key parameters such as thermal range, vapour pressure, and extent of decomposition during TGA into account to quantitatively compare precursors (Table 5.1). ${ }^{81,332}$ A positive figure of merit indicates the suitability of a precursor while negative indicates a poor candidate. With $\sigma=35$, complex 4 Co ranks between $\operatorname{Co}(\mathrm{dad})_{2}(\sigma=64)$ and $\mathrm{CoCl}_{2}$ (tmeda) $(\sigma=-2)$ where the weak performance of the latter originates from its low volatility. Therefore, 4Co was not only competitive with successfully applied Co(II) precursors but, to the best of our knowledge, represents the only known potential Co(IV) precursor candidate.

The most important characteristic of an ALD precursor is the ability to form a stable self-limiting monolayer on a surface. ${ }^{332}$ After all reactive surface sites have undergone a reaction with precursor molecules, no further adsorption should occur, allowing conformal and uniform atomic layer deposition to take place during the next self-limiting 
Table 5.1: Thermal data and figure of merit $\sigma$ for Co precursors.

\begin{tabular}{cccccc}
\hline Complex & $\begin{array}{c}T_{D} \\
{ }^{\circ} \mathrm{C}\end{array}$ & $\begin{array}{c}T_{V} \\
{ }^{\circ} \mathrm{C}\end{array}$ & $\begin{array}{c}\Delta T \\
{ }^{\circ} \mathrm{C}\end{array}$ & $\begin{array}{c}m_{\text {res }} \\
\%\end{array}$ & $\sigma$ \\
\hline 4Co & 197 & 150.4 & 46.6 & 3.2 & 35.0 \\
$\mathrm{CoCl}_{2}$ (tmeda) & 235 & 215.9 & 19.3 & 26.2 & -1.8 \\
$\mathrm{Co}(\text { dad })_{2}$ & 219 & 145.8 & 72.9 & 1.8 & 64.1 \\
\hline
\end{tabular}

reaction. ${ }^{333}$ Employing a quartz crystal microbalance (QCM) in our home-built ALD tool, we investigated the potential for $\mathbf{4 C o}$ to fulfill this basic yet critical requirement. The precursor was heated to $135^{\circ} \mathrm{C}$ for delivery and the QCM crystal was heated to $150{ }^{\circ} \mathrm{C}$ for saturation experiments. Compound 4Co demonstrated self-limiting adsorption on alumina with a mass gain of $(49.2 \pm 0.3) \mathrm{ng} \mathrm{cm}^{-2}$ that was stable over roughly 35 minutes (Figure 5.6c). This encouraging initial result motivated us to begin developing ALD processes with $\mathbf{4 C o}$ in ongoing research.

\subsection{Conclusion}

In summary, a thermally stable, volatile, homoleptic, spirocyclic $\mathrm{Co}(\mathrm{IV})$ bis( $\beta$-silyldiamide) was synthesized in a facile one-step salt-metathesis exploiting $\mathrm{Co}$ (II) disproportionation. Experiment and theory confirmed that it exhibits a rare low-spin $\mathrm{d}^{5}$ doublet configuration enforced by the steric bulk and geometric rigidity of its ligands. The remarkable stability of 4Co makes it a promising precursor for vapour deposition, and its ability to form a stable self-limiting monolayer makes it a promising ALD precursor. Beyond this, $\mathbf{4 C o}$ represents a simple, inexpensive, and accessible starting material for further high-valent $\mathrm{Co}(\mathrm{IV})$ chemistry to be explored. 


\subsection{Experimental Details}

\subsubsection{General Considerations}

All manipulations were performed under air-free conditions using standard Schlenk techniques or in a $\mathrm{N}_{2}$-filled (99.998\% purity) MBraun Labmaster 130 drybox $\mathrm{CoCl}_{2}$ (anhydrous, 99.9\%), $N, N, N^{\prime}, N^{\prime}$-tetramethylethylene-1,2-diamine (tmeda, 99.5\%), dichlorodimethylsilane (98.5\%), ${ }^{n} \mathrm{BuLi}$ (2.0 $\mathrm{M}$ in hexanes) and tert-butylamine (99.5\%) were purchased from Millipore-Sigma and used as received. Toluene, hexanes, pentane, diethyl ether and THF were ACS Reagent Grade, purified by an MBraun Solvent Purifier System, and stored over $4 \AA$ molecular sieves for more than a day before use. Deuterated benzene $\left(\mathrm{C}_{6} \mathrm{D}_{6}\right)$ was purchased from Millipore-Sigma and degassed by freeze-pump procedure prior to

use. $(\mathrm{N}(\mathrm{H}) \mathrm{tBu})_{2} \mathrm{SiMe}_{2},{ }^{289}(\mathrm{~N}(\mathrm{Li}) \mathrm{tBu})_{2} \mathrm{SiMe}_{2},{ }^{290} \mathrm{CoCl}_{2}(\mathrm{tmeda})^{334,334}$ and $\mathrm{Co}(\mathrm{dad})_{2}{ }^{335}$ (dad $=N, N^{\prime}$-di-tert-butyl-2,3-diazabutadienyl) were synthesized following literature procedures.

${ }^{1} \mathrm{H}$ Nuclear Magnetic Resonance (NMR) spectra were recorded on a Bruker Avance II $300 \mathrm{MHz}$ instrument. Electron impact mass spectrometry (EI-MS) was performed with a Kratos Concept magnetic sector electron impact mass spectrometer in high-resolution mode. Elemental analysis was conducted with a Vario Micro Cube elemental analysis tool (Elementar Analysensysteme) in CHNS analysis mode. Magnetic susceptibility measurements were conducted on a Johnson Mattheys Mark I balance. UV/VIS measurements were obtained on a Varian Cary 3e UV-Visible spectrophotometer. IR spectroscopic measurements were performed on an Agilent Cary 630 FTIR instrument equipped with a diamond attenuated-total-reflectance (ATR) unit. EPR spectra were recorded on a Bruker EMXplus X-band EPR spectrometer. Powder X-ray diffractometry (PXRD) of byproduct from the synthesis of $\mathbf{4 C o}$ was carried out on a Rigaku Ultima IV diffractometer [X-ray source $\mathrm{Cu} \mathrm{K} \alpha(\lambda=1.54184 \AA), 40 \mathrm{kV}, 30 \mathrm{~mA}]$ and found to contain metallic $\mathrm{Co}(0)$. 


\subsubsection{Synthesis of $\mathrm{Co}\left[\left(\mathrm{N}^{t} \mathrm{Bu}\right)_{2} \mathrm{SiMe}_{2}\right]_{2}(4 \mathrm{Co})$}

$\mathrm{CoCl}_{2}$ (tmeda) (2.95 g, $\left.12 \mathrm{mmol}\right)$ was suspended in $50 \mathrm{ml}$ of pentane and stirred for 30 minutes. Then, a solution of $\left[\mathrm{N}(\mathrm{Li})^{t} \mathrm{Bu}\right]_{2} \mathrm{SiMe}_{2}(2.57 \mathrm{~g}, 12 \mathrm{mmol})$ in $40 \mathrm{ml}$ of pentane was added via syringe over 10 minutes at room temperature (RT, ca. $21^{\circ} \mathrm{C}$ ), which caused a colour change from the blue $\mathrm{CoCl}_{2}$ (tmeda) suspension to a dark brown-black suspension was observed. The reaction mixture was then stirred at RT overnight (ca. $16 \mathrm{~h}$ ). Besides the formation of $\mathrm{LiCl}$, sticky and finely dispersed particles were deposited on the reaction flask walls. The reaction mixture was filtered to remove the precipitate, solvent and tmeda were then removed from the filtrate under vacuum, and the crude product was further dried under vacuum for $2 \mathrm{~h}$. The crude product can be purified recrystallization and, more preferably, sublimation. Crystallization: due to its silylamide ligand, compound 4Co possesses excellent solubility in commonly used polar and non-polar solvents such as hexanes, pentane, toluene, diethyl ether and THF which makes recrystallization difficult. Yet, crystallization from a minimal amount of pentane proved to be repeatably successful given the solution was stored at $-49^{\circ} \mathrm{C}$ for $96 \mathrm{~h}$, giving brown-black crystals $(1.08 \mathrm{~g}$, $40.4 \%$ based on $\left[\mathrm{N}(\mathrm{Li})^{t} \mathrm{Bu}\right)_{2} \mathrm{SiMe}_{2}$. Sublimation: after drying, the crude product was finely ground in a mortar and pestle, then added to a Schlenk flask fitted with a water-cooled sublimation cold-finger. The product was then sublimed $\left(85^{\circ} \mathrm{C} / 10 \mathrm{mTorr}\right)$ and obtained as a black crystalline solid (1.73 g, 64.8\% based on ligand). ${ }^{1} \mathrm{H}$ NMR $\left(300 \mathrm{MHz}, \mathrm{C}_{6} \mathrm{D}_{6}\right): \delta(\mathrm{ppm})$ $=-0.32\left(s, 12 \mathrm{H},\left(\mathrm{Si}\left(\mathrm{CH}_{3}\right)_{2}\right)\right), 17.77\left(s, 36 \mathrm{H}, \mathrm{N}-\mathrm{C}\left(\mathrm{CH}_{3}\right)_{3}\right) . \mathrm{EA}$ calcd $(\%): \mathrm{C} 52.25, \mathrm{H} 10.52, \mathrm{~N}$ 12.19; found (\%): C 52.34, H 10.60, N 12.11. HRMS (EI) for the molecular ion peak [M]+: Found, $\mathrm{m} / \mathrm{z}=459.2739$, calcd. 459.2750 , dev. $-0.54 \%$. EI-MS $(70 \mathrm{eV}) \mathrm{m} / \mathrm{z}(\%)$ [fragment]: $459.3(46.5 \%)[\mathrm{M}]^{+}, 444.3(80.3 \%)\left[\mathrm{M}-\mathrm{CH}_{3}\right]^{+}, 429.2(36.6 \%)\left[\mathrm{M}-2 \mathrm{CH}_{3}\right]^{+}, 414.2(100 \%)$ $\left[\mathrm{M}-3 x \mathrm{CH}_{3}\right]^{+}, 200.1(20.8 \%)[\mathrm{L}]^{+}, 199.6(67.7 \%)\left[\mathrm{M}-\mathrm{L}-4 \mathrm{x}-\mathrm{CH}_{3}\right]^{+}, 114.1(17.9 \%)[\mathrm{L}-$ $\left.\mathrm{NC}\left(\mathrm{CH}_{3}\right)_{3}-\mathrm{CH}_{3}\right]^{+}$. Where $\mathrm{M}=$ title complex, $\mathrm{L}=$ Diamido ligand. IR in ATR mode: $v / \mathrm{cm}^{-} 1$ = $2958.6(\mathrm{~m}), 2921.3(\mathrm{~m}), 2890.4(\mathrm{~m}), 2856.6(\mathrm{~m}), 1453.3(\mathrm{~m}), 1378.7(\mathrm{w}), 1350.3(\mathrm{~m}), 1241.3$ 
(m), $1225.8(\mathrm{~m}), 1193.6(\mathrm{~s}), 1088.4(\mathrm{~s}), 1040.8(\mathrm{w}), 1015.6(\mathrm{w}), 963.9(\mathrm{w}), 911.6(\mathrm{w}), 825.8(\mathrm{~s})$, $756.5(\mathrm{~s}), 685.4(\mathrm{~s}), 663.1(\mathrm{~s})$. UV/VIS (toluene): $\lambda_{\max }=320.1 \mathrm{~nm}$. Magnetic moment: $\mu_{\mathrm{eff}}=$ $1.8 \pm 0.1 \mu \mathrm{B}$.

The precipitation of metallic $\mathrm{Co}(0)$ from the synthesis was corroborated with PXRD as follows. A $100 \mathrm{mg}$ sample of the byproduct from the above synthesis was washed with 5 portions of $10 \mathrm{ml}$ of THF in a glove box and subsequently dried to remove LiCl. A greyblack powder remained which was carefully filled in a glass capillary. The capillary was sealed with grease, flame-sealed, and subsequently mounted on a rotatory sample stage. PXRD was conducted in Bragg-Brentano geometry in a $2 \theta$ range of $20-60^{\circ}$.

\subsubsection{Single-Crystal X-Ray Diffraction (XRD)}

Crystals of compound 4Co suitable for single-crystal X-ray diffraction (SCXRD) were grown from a toluene solution of sublimed material slowly cooled to $-49^{\circ} \mathrm{C}$. The crystal chosen was attached to the tip of a MicroLoop with Paratone-N oil. Measurements were made on a Bruker D8 VENTURE diffractometer equipped with a PHOTON III CMOS detector using monochromated Mo $\mathrm{K} \alpha$ radiation $(\lambda=0.71073 \AA)$ from an Incoatec microfocus sealed tube at $100 \mathrm{~K}$. The initial orientation and unit cell were indexed using a leastsquares analysis of the reflections collected from a complete $360^{\circ} \varphi$-scan with $1^{\circ}$ per frame. For data collection, a strategy was calculated to maximize data completeness and multiplicity, in a reasonable amount of time, and then implemented using the Bruker Apex 3 software suite.The crystal to detector distance was set to $4 \mathrm{~cm}$. Data collection, unit cell refinement, data processing and multi-scan absorption correction were applied using the APEX3 software package. The structures were solved using SHELXT ${ }^{336}$ and all non-hydrogen atoms were refined anisotropically with SHELXL ${ }^{337}$ using a combination of shelXle ${ }^{338}$ and OLEX2 ${ }^{339}$ graphical user interfaces. Unless otherwise noted, all hydrogen atom positions were idealized and ride on the atom to which they were attached. The final refinement included anisotropic displacement factors on all non-hydrogen atoms. 
CSD entry 1977041 contains the supplementary crystallographic data for complex 4Co. These data can be obtained free of charge from the Cambridge Crystallographic Database Centre via https://summary.ccdc.cam.ac.uk/structure-summary-form.

\subsubsection{Thermogravimetric Analysis (TGA)}

TGA was performed with a TA Instruments Q50 kept in an MBraun Labmaster 130 drybox filled with $\mathrm{N}_{2}$. Pt pans were coated before each experiment with approximately $55 \mathrm{~nm}$ of $\mathrm{Al}_{2} \mathrm{O}_{3}$ by atomic layer deposition (ALD) in a Picosun R-200 reactor using 500 cycles of trimethylaluminum (TMA) and water at $200{ }^{\circ} \mathrm{C}$ with $0.1 \mathrm{~s}$ pulses and $8 \mathrm{~s}$ purges each. This is critical to protect the Pt pans as they become brittle and break easily after hightemperature exposure to $\mathrm{Pb}(0)$ residues from precursor decomposition. The TGA furnace was purged with $99.999 \% \mathrm{~N}_{2}$ at $60 \mathrm{sccm}$ and the temperature was increased at $10{ }^{\circ} \mathrm{C} \mathrm{min}-1$ for the "ramp" experiments, and increased at $40{ }^{\circ} \mathrm{C} \mathrm{min}^{-1}$ to increase temperatures stepwise to hold at isotherms of $60-130^{\circ} \mathrm{C}$ for $7 \mathrm{~min}$ each for the "stepped isothermal" experiments. The exact masses for the $10 \mathrm{mg}, 20 \mathrm{mg}$, and $40 \mathrm{mg}$ loadings shown in Figure 2.10 were $11.61886 \mathrm{mg}, 20.40275 \mathrm{mg}$, and $39.35869 \mathrm{mg}$, respectively.

\subsubsection{Differential Scanning Calorimetry (DSC)}

Differential scanning calorimetry (DSC) was performed with a TA Instruments Q10 instrument. DSC samples with mass loadings of ca. $(0.30 \pm 0.03) \mathrm{mg}$ were sealed in aluminum pans inside a glovebox prior to analysis. DSC experiments on complex 4Co with approximately equal mass loadings were repeated in triplicate to ensure the validity of the recorded data. Unless otherwise stated all samples were heated to $400{ }^{\circ} \mathrm{C}$ at a ramp rate of $10{ }^{\circ} \mathrm{C} \mathrm{min}^{-1}$ with nitrogen $(99.998 \%$ purity) as the purge gas. The onset of decomposition $\left(T_{D}\right)$ was defined as a $5 \%$ increase in exothermic heat flow from the beginning of the exothermic event. TGA and DSC experiments on $\mathrm{CoCl}_{2}$ (tmeda) and [Co(DAD)2] were performed on the same instruments with identical methodology. 


\subsubsection{Figure of Merit $\sigma$}

TGA and DSC data were compared by computing the dimensionlesss figure of merit $\sigma$ assuming decomposition to $\mathrm{Co}(0)$ (Equation 5.1). ${ }^{81}$

$$
\sigma=\left(1-\frac{m_{\mathrm{res}}}{m_{\mathrm{Co}(0)}}\right)\left(T_{D}-T_{V}\right)
$$

where $m_{\text {res }}$ is the residual mass from TGA and $m_{\mathrm{Co}(0)}$ is the mass of cobalt in the complex, usually in \%; and $T_{D}-T_{V}$ is the "thermal range" between the onset of decomposition $T_{D}$ and the 1 Torr vapour pressure temperature $T_{V}$. Negative figure of merit indicates poor long-term thermal stability at temperatures necessary for the precursor to be evaporated for ALD (i.e., $T_{D}<T_{V}$ ) or substantial decomposition to something other than the pure element (e.g., $\mathrm{Co}_{x} \mathrm{~N}_{y}$ ). The obtained figures of merit are summarized in Table 5.1.

\subsubsection{Quartz Crystal Microbalance (QCM) Saturation Experiments}

ALD saturation experiments were conducted in a home-built low-pressure hot-walled ALD reactor equipped with a quartz crystal microbalance (QCM) (COLNATEC, EON-LT) and an integrated thermocouple. Gold-coated QCM crystals (PhillipTech, ca. $6 \mathrm{MHz}, d=$ $14 \mathrm{~mm}$ ) were mounted on a holder that was inserted into the chamber and preheated for $2 \mathrm{~h}$ prior to precursor exposure to allow the temperature and frequency of the crystal to

equilibrate. Experiments were conducted at a temperature of $150^{\circ} \mathrm{C}$. For each run, ca. $1 \mathrm{~g}$ of compound $4 \mathrm{Co}$ was loaded into a stainless steel bubbler and heated to $135^{\circ} \mathrm{C}$. During the exposure tests, nitrogen $(99.998 \%, 50 \mathrm{sccm})$ was flowed through the headspace of the bubbler to deliver the vapour of complex $4 \mathrm{Co}$ to the reactor chamber. In initial runs, the bare QCM crystal surface (i.e., $\mathrm{Au}$ ) was used without modification, but no significant frequency change (i.e., no adsorption) occurred after 5 minutes. In subsequent runs, we coated the QCM crystals in situ with an approximately $11 \mathrm{~nm}$ thin film of $\mathrm{Al}_{2} \mathrm{O}_{3}$ by ALD with trimethylaluminum (TMA) and $\mathrm{H}_{2} \mathrm{O}$ at $150{ }^{\circ} \mathrm{C}$. TMA and $\mathrm{H}_{2} \mathrm{O}$ were fed into the 
reactor system from stainless-steel ampules by simple vapour draw into lines constantly purged with $50 \mathrm{sccm}$ of $\mathrm{N}_{2}$ (99.999\%). 100 cycles of the following pulse sequence were applied: $0.1 \mathrm{~s}$ TMA, $10 \mathrm{~s} \mathrm{~N}_{2}$ purge, $0.1 \mathrm{~s} \mathrm{H}_{2} \mathrm{Os}, 10 \mathrm{~s} \mathrm{~N}_{2}$ purge. After ALD of $\mathrm{Al}_{2} \mathrm{O}_{3}$, the QCM crystal was allowed to equilibrate for 30 minutes before a saturation experiment with 4Co was started. Saturation experiments were then conducted with long exposure times of up to 35 minutes to demonstrate the self-limiting character of adsorption. The mass gain $(\Delta m)$ was obtained using the Sauerbrey equation (Equation 5.2). ${ }^{340}$

$$
\Delta m=-\frac{2 f_{0}^{2}}{A \sqrt{\rho_{q} \mu_{q}}} \Delta f
$$

where $\Delta f$ is the change in frequency, $f_{0}$ is the fundamental resonsant frequency, $A$ is the surface area between the QCM electrodes $\left(x \mathrm{~cm}^{2}\right), \rho_{q}$ is the density of quartz $\left(2.64 \mathrm{~g} \mathrm{~cm}^{-3}\right)$, and $\mu_{q}$ is the shear wave velocity of AT-cut quarts $\left(3.34 \times 10^{-5} \mathrm{~cm} \mathrm{~s}^{-1}\right)$.

\subsubsection{Computational Details}

Initial density functional theory (DFT) calculations for $\mathbf{4 C o}$ and $\mathrm{Co}(\text { nor })_{4}$ were performed with Gaussian 16 Revision C.01 (G16). ${ }^{341}$ DFT was used to optimize the geometries of 4Co in its doublet, quartet, and sextet spin states (i.e., multiplicities of 2, 4, and 6). Initial geometries were taken from the solid-state structures (XRD, vide supra, and, ${ }^{322}$ respectively). Then, we optimized the structures using the open-shell unrestricted PerdewBurke-Ernzerhof (PBE) exchange-correlation functional ${ }^{342}$ (uPBEPBE keyword in G16), Ahlrichs's double- $\zeta$ quality split valence basis set def2-SVP on all atoms, ${ }^{241}$ neutral charge, and ultrafine integral grid. These were then used as starting geometries and optimized with the APFD (only 4Co), ${ }^{343}$ B97-D3(BJ), ${ }^{344}$ PW6B95-D3(BJ) hybrid, ${ }^{297}$ and wB97X-D2 ${ }^{345}$ range-separated hybrid functionals using the same prior other settings. Dispersion forces were included with the empirical corrections according to the Grimme ${ }^{239}$ with BeckeJohnson damping $[\mathrm{D} 3(\mathrm{BJ})],{ }^{240}$ All geometries were confirmed to be minima on the po- 
tential energy surface (PES) by ensuring they had no imaginary frequency modes. Finally, we performed single point energy calculations using Ahlrichs's quadruple- $\zeta$ with polarization basis set def2-QZVPP to approach the basis set limit and minimize any basis set superposition error (BSSE).

EPR $g$-tensor calculations were performed with ORCA v4.2.1 ${ }^{346}$ using open-shell unrestricted DFT for the doublet, quartet, and sextet spin states of 4Co (def2-QZVPP//PW6B95$\mathrm{D} 3(\mathrm{BJ}) /$ def2-SVP) using geometries taken from the previous calculations (vide supra). Since there were no heavy atoms, only the isotropic and dipolar parts of the hyperfine coupling (HFC, aiso and adip keywords) were considered for all nuclei in the $g$-tensor calculations. A benchmark calculation for the doublet state including spin-orbit coupling (aorb keyword) was performed, which gave identical $g$-tensor values as the calculations without it, verifying the trustworthiness of the previous calculations.

The poor performance of DFT to predict the ground spin-state of $\mathbf{4 C o}$ prompted us to explore other functionals and more accurate post-HF methods with ORCA v4.2.1. We explored the effect of the added fraction of Hartree-Fock (HF) exchange in hybrid functionals by employing their corresponding "pure" density functionals that omit it. Thus, complex $4 \mathrm{Co}$ in the doublet and quartet ground state was reoptimized with the def2-SVP basis set and the following "pure" generalized-gradient approximation (GGA) functionals: Perdew-Burke-Ernzerhof exchange and correlation (PBE) ${ }^{342}$ Becke exchange ${ }^{347}$ with Lee-Yang-Parr correlation ${ }^{348}$ (BLYP), and Handy-Cohen optimized exchange ${ }^{349,350}$ (OPTX) with LYP (OLYP), and with PBE correlation (OPBE) ${ }^{351}$ Convergence criteria were tighted to ensure accuracy using the VeryTightSCF and VeryTightOpt keywords with Grid5 and FinalGrid7 integral grid sizes. The resolution of identity for Coulomb integrals and chainof-spheres numerical integration for HF exchange (RIJCOSX keyword) approximation ${ }^{352}$ with a large grid size (GridX7) was used to speed up the SCF step for the hybrid functionals. Numerical frequency calculations (NumFreq keyword) were performed to confirm they were minima on the PES, and to avoid a known bug in ORCA involving some func- 
tionals, RIJCOSX and analytical Hessians (Freq keyword). Optimizations were followed by single-point calculations with a large basis set (def2-QZVPP) for improved accuracy of the electronic energy. The pure functionals all correctly identified the doublet state as the preferred configuration, and the consistently excellent performance of the OPBE functional in the literature and its simple implementation in ORCA encouraged us to trust its results for a qualitative molecular orbital analysis. Unrestricted natural orbitals (UNOs) were generated with the UNO keyword in ORCA.

Less sterically encumbered $\mathrm{N}$-substituted analogues of $4 \mathrm{Co}$ were also investigated with ORCA v4.2.1. The structures were optimized using unrestricted DFT (def2-QZVPP// PW6B95-D3(BJ)/def2-SVP) using the RIJCOSX approximation with the substituents $\mathrm{R}=$ $-\mathrm{CH}_{3},-\mathrm{CH}_{2} \mathrm{CH}_{3}$, and $-\mathrm{CH}\left(\mathrm{CH}_{3}\right)_{2}$. Starting geometries were obtained by modifying the structure of $4 \mathrm{Co}$ with Avogadro v1.4, replacing $\mathrm{CH}_{3}$ moieties with $\mathrm{H}$ on the tert-butyl groups and then optimizing the geometries using the same settings as with 4Co above. The doublet state for the $\mathrm{R}=-\mathrm{CH}_{3}$ analogue could not be optimized to a minimum on the PES (three small negative frequencies (ca. $50 \mathrm{~cm}^{-1}$ ) could not be eliminated with tighter convergence or optimization criteria) and was ignored during subsequent post-HF calculations.

Domain based local pair natural orbital coupled cluster calculations with singles, doubles, and perturbative triples [DLPNO-CCSD(T) keyword] ${ }^{298}$ were performed with ORCA v4.2.1 using an unrestricted Hartree-Fock (UHF) reference wavefunction (VeryTightSCF keyword) and the def2-TZVPP basis set. This method was applied using geometries obtained from DFT (PW6B95-D3(BJ)/def2-SVP) due to their close resemblance with the experimental solid-state structure. Due to poor convergence, UHF wavefunctions were first calculated using a double- $\zeta$ (def2-SVP) basis set, and those orbitals were used as the starting point for the triple- $\zeta$ reference wavefunction calculations (def2-TZVPP). Quasirestricted orbitals (QROs) were used to eliminate spin contamination, ${ }^{353}$ which is the default setting in ORCA. 
Wavefunction analysis was performed with Multiwfn v3.7 $7^{299}$ running on a personal computer using wavefunction data from G16 ( ${ }^{*}$.fchk format) and ORCA v4.2.1 ( ${ }^{*}$.molden.input format) Analysis performed and included here: spin density isosurface of 4Co and Co(nor) $)_{4}$; Mayer bond order (MBO), ${ }^{300}$ Bader's quantum topology of atoms-in-molecules (QTAIM), ${ }^{301,302}$ and Johnson's non-covalent interaction (NCI) analysis; ${ }^{276}$ and generation of orbital cube files. Graphics were drawn with UCSF Chimera ${ }^{150}$ and VMD, ${ }^{354}$ with editing and postprocessing done in Adobe Photoshop 2020. All Gaussian and ORCA calculations were performed on the Cedar cluster maintained by Compute Canada and housed at Simon Fraser University. All structures, energies, orbitals, and wavefunctions are available upon request. 


\section{Chapter 6}

\section{Volatile and Thermally Stable Polymeric Tin Trifluoroacetates}

Reprinted and adapted from the original article published as:

Goran Bačić, ${ }^{c}$ Conor D. Rankine, ${ }^{y}$ Jason D. Masuda, ${ }^{m}$ Derek A. Wann, ${ }^{y}$ and Seán T. Barry. ${ }^{c}$ Volatile and Thermally Stable Polymeric Tin Trifluoroacetates, Inorg. Chem., 2020, 59, 2, 996-1005. Copyright 2020 American Chemical Society.

c Department of Chemistry, Carleton University, 1125 Colonel By Drive, Ottawa, Ontario, K1S 5B6, Canada

$y$ Department of Chemistry, University of York, Heslington, York, YO10 5DD, United Kingdom

$m$ Department of Chemistry, Saint Mary's University, 923 Robie Street, Halifax, Nova Scotia, B3H 3C3, Canada 


\section{Abstract}

Tin trifluoroacetates are effective vapour phase single-source precursors for F-doped $\mathrm{SnO}_{2}$, but their structures have been poorly understood for decades. Here we undertook a comprehensive structural analysis of these compounds in both the solid and gas phases through a combined single-crystal X-ray crystallography (XRD), gas phase electron diffraction (GED), and density functional theory (DFT) investigation. Tin(II) bis(trifluoroacetate) (1) thermally decomposes into a 1:1 mixture of $\mathbf{1}$ and ditin(II)- $\mu$-oxy-bis- $\mu$-tri-fluoroacetate (2) during sublimation, which then polymerize into hexatin(II)-di- $\mu_{3}$-oxy-octakis- $\mu$-trifluoroacetate (3) upon solidification. Reversible depolymerization occurred readily upon heating, making 3 a useful vapour phase precursor itself. Tin(IV) tetrakis(trifluoroacetate) (5) was also found to be polymeric in the solid state, but it evaporated as a monomer over $130{ }^{\circ} \mathrm{C}$ lower than 3 . This counterintuitive improvement in volatility by polymerization was possibly due to the large entropy change during sublimation, which offers a strategic new design feature for vapour phase deposition precursors. 


\subsection{Introduction}

Demand for transparent conductors made entirely of earth-abundant elements like Fdoped $\mathrm{SnO}_{2}$ is rising due to economic and environmental concerns. ${ }^{22,355-357}$ Atomic layer deposition (ALD) and chemical vapour deposition (CVD) are attractive high performance methods for their fabrication, and have become prevalent in industry. ${ }^{41,70}$ However, there are currently no ALD processes to deposit F-doped $\mathrm{SnO}_{2}$, likely because fluorine doping agents are hazardous (e.g., HF) ${ }^{358,359}$ or are difficult to use (e.g., metal fluorides, decomposition of fluorinated ligands). ${ }^{70,360,361}$ We set out to evaluate fluorinated single-source precursors with the hopes of developing a simple ALD process that can be integrated into real-world device architectures.

Trifluoroacetic acid $\left(\mathrm{HO}_{2} \mathrm{CCF}_{3}\right)$ is inexpensive, non-toxic, and environmentally benign, ${ }^{362}$ and tin(II) bis(trifluoroacetate) (1) is known to be one of the best precursors for Fdoped $\mathrm{SnO}_{2}$ by CVD. ${ }^{363-365}$ Highly transparent films with low resistivities were deposited with high growth rates using $\mathbf{1}$ and air at atmospheric pressure above $200{ }^{\circ} \mathrm{C}$. However, compound 1 has only been reported once, and was prepared by condensing blue-black tin(II) oxide with an excess of trifluoroacetic acid and trifluoroacetic anhydride. ${ }^{366}$ Additionally, it has only characterized by microanalysis, FTIR and ${ }^{119} \mathrm{Sn}$ Mossbauer spectroscopy; while structural investigations were hindered by polymerization, thermal decomposition, and oxidation. ${ }^{366,367}$ Despite their simple composition, tin trifluoroacetates have seen only a handful of structural analyses over the last three decades. ${ }^{366-369} \mathrm{We}$ wished to gain a fundamental insight into the structure-property relationships of tin trifluoroacetates to better understand their suitability as vapour phase precursors.

Traditionally, oligomers and polymers were presumed to be non-volatile due to their extreme molecular weight, ${ }^{370}$ and great lengths were usually taken to ensure that precursors are monomeric by design ${ }^{85}$ While some volatile CVD precursors were coincidentally polymeric, ${ }^{371}$ the features that made some polymers volatile has not been described. Here 
we prepare and evaluate tin trifluoroacetates as potential vapour phase precursors under realistic conditions. Our results show that polymerization can be exploited to dramatically and counterintuitively improve volatility, possibly by a large change in entropy during evaporation from a polymer to monomers. The high volatility and thermal stability of these simple fluorinated compounds, especially compared to their hydrogenated analogues, make them promising precursors for vapour deposition of F-doped $\mathrm{SnO}_{2}$.

\subsection{Results and Discussion}

\subsubsection{Tin(II) Trifluoroacetates}
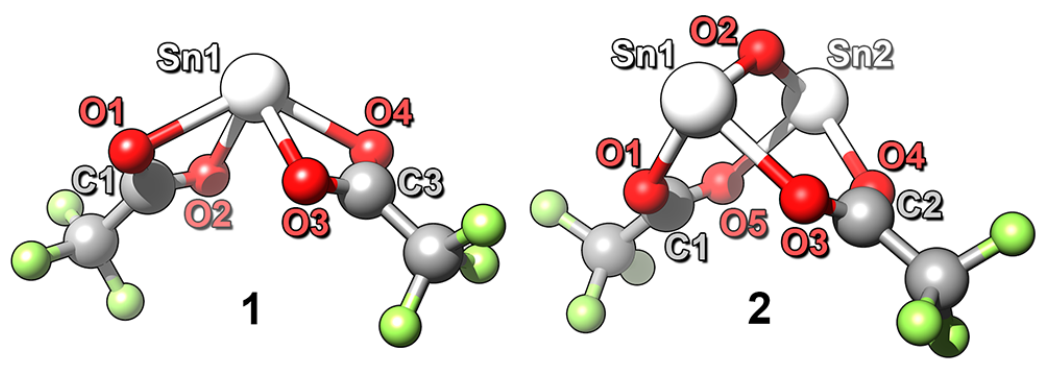

Figure 6.1: Gas phase products of tin(II) bis(trifluoroacetate) (1, left) and ditin(II) $\mu$-oxybis( $\mu$ trifluoroacetate) (2, right).

While trying to make tin(II) bis(trifluoroacetate) (1) by oxidation of $\mathrm{Sn}(0)$ with copper(II) bis(trifluoroacetate) in deoxygenated water (Equation 6.1), ${ }^{372}$ we obtained a colorless powder after prolonged drying under high vacuum. From this crude product we were able to sublime a colorless powder $\left(150{ }^{\circ} \mathrm{C}, 10 \mathrm{mTorr}\right)$ without any non-volatile residue, which itself could be sublimed and recovered quantitatively. The crude yield of this synthesis was moderate (ca. 65\%) assuming conversion to 1 . However, both mass spectrometry and NMR spectroscopy were not consistent with the formulation of $\mathbf{1}$, but rather another unknown species. A combined density functional theory (DFT) and gas phase electron diffraction (GED) approach was used to unambiguously determine the species 
that evolved during sublimation and by extension infer the composition of the unknown solid. Our initial structural guesses were guided by electron impact mass spectrometry (EIMS), and we determined that there was an equal mixture of $\mathbf{1}$ and ditin(II)- $\mu$-oxy-bis- $\mu$ trifluoroacetate (2) in the gas phase by the exceptional agreement between our theoretical and GED data (Figures 6.1 and 6.2).

$$
\begin{aligned}
& \mathrm{Sn}(0)+\mathrm{Cu}\left(\mathrm{O}_{2} \mathrm{C}_{2} \mathrm{~F}_{3}\right)_{2} \longrightarrow \mathrm{Sn}\left(\mathrm{O}_{2} \mathrm{CCF}_{3}\right)_{2}(\mathbf{1})+\mathrm{Cu}(0) \downarrow \\
& 3 \mathrm{Sn}\left(\mathrm{O}_{2} \mathrm{CCF}_{3}\right)_{2} \stackrel{\Delta}{\longrightarrow} \mathbf{1}+\mathrm{Sn}_{2} \mathrm{O}\left(\mathrm{O}_{2} \mathrm{CCF}_{3}\right)_{2}(2)+\mathrm{O}\left(\mathrm{OCCF}_{3}\right)_{2} \uparrow \\
& \mathbf{1}+\mathbf{2} \rightleftharpoons \frac{1}{2} \mathrm{Sn}_{6} \mathrm{O}_{2}\left(\mathrm{O}_{2} \mathrm{CCF}_{3}\right)_{8}(\mathbf{3})
\end{aligned}
$$

The unknown solid was also isolated during the sublimation of $\mathbf{1}$ prepared by the previously reported method, ${ }^{366}$ which was also confirmed by NMR, EIMS, and GED. A single crystal suitable for X-ray crystallography was grown by slowly cooling a doubly sublimed sample of the unknown solid in toluene. Upon structural determination, we confirmed it was in fact the polymeric hexatin(II) di- $\mu_{3}$-oxy-octakis- $\mu$-trifluoroacetate (3, Figure 6.3), which had a stoichiometry that equaled a mixture of $\mathbf{1}$ and 2 (Equation 6.3). Thermal depolymerization of $\mathbf{3}$ into $\mathbf{1}$ and 2 was reversible, and 3 was recovered quantitatively after repeated sublimations. If the material isolated following both synthetic methods before heating under vacuum was in fact $\mathbf{1}$, it is likely that $\mathbf{1}$ decomposes by eliminating trifluoroacetic anhydride during sublimation to form gaseous 1 and 2 (Equation 6.2), which polymerize

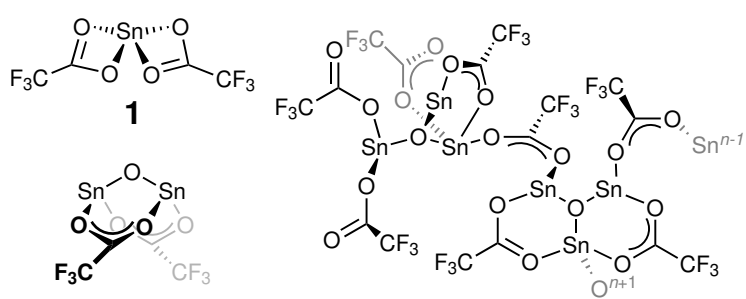

2

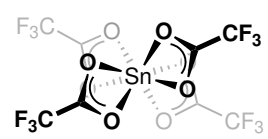

6
3

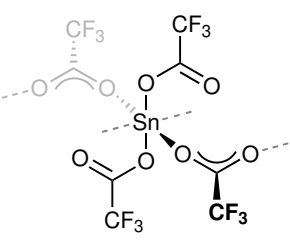

5 into 3 upon solidification (Equation 6.3). The oxidation of metallic tin was easily scalable, and multigram batches ( $>25 \mathrm{~g}$ ) were routinely prepared in good yields. Benign metal 
waste, mild reaction conditions, and simple workup make it a convenient green synthetic protocol $^{373}$ and the most promising route to prepare 3 .
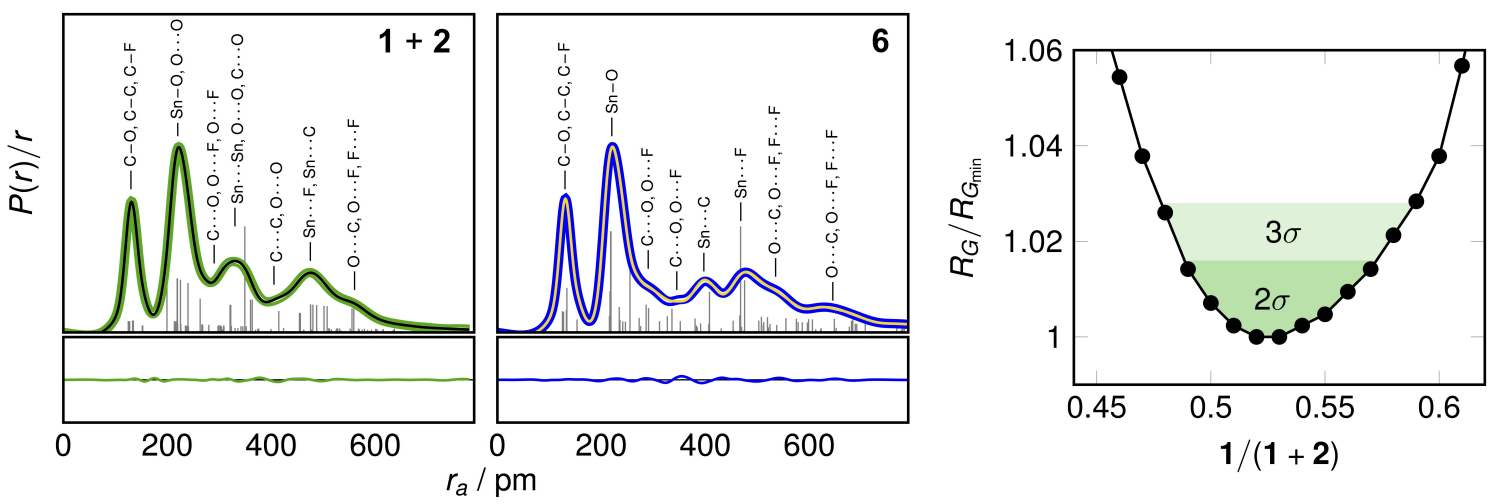

Figure 6.2: Gas-phase electron diffraction (GED) radial distribution curves (RDC, top) and difference curves (bottom) for 1+2 (left) and 6 (middle). Experimental data for 1+2 (left, black with green highlight) were compared to theoretically calculated RDCs for different molar ratios of 1 and 2 (white), and the best fit at 52:48 (95\% confidence) was plotted here (RG $=4.2 \%$ and $\mathrm{RD}=$ $2.9 \%$ ). Similarly, 6 (middle, white with blue highlight) was compared to its DFT optimized structure (gold) and found to be monomeric 6 in the gas phase ( $\mathrm{RG}=5.0 \%$ and $\mathrm{RD}=3.7 \%$ ). (right) Quality of least-squares refinement RG/RGmin of GED data of $\mathbf{3}$ as a function of the mole fraction of $\mathbf{1}$ and 2 . The minimum is between $0.52-0.53$, and 0.500 lies within $2 \sigma$ of the minimum (i.e., within 95\% confidence). This confirmed bulk 3 thermally depolymerizes into an equimolar mixture of $\mathbf{1}$ and 2 upon evaporation.

In the solid state, asymmetric units of 3 were linked by covalent bonds between Sn 5 on one and $\mathrm{O} 18^{*}$ on another $(2.360(3) \AA)$, making each a monomer within an extended polymeric chain (Figure 6.4). These chains packed in a crystal structure with longer $\mathrm{Sn}-\mathrm{O}_{2} \mathrm{CCF}_{3}$ intra- and intermolecular coordination where the $\mathrm{Sn}(\mathrm{II})$ atoms formed distinct and extremely distorted pseudo-square pyramidal geometries (Figure 6.8). We could glean no obvious trend from the coordination geometries. The plastic behaviour of 3 in the solid state, its facile thermal depolymerization into $\mathbf{1}$ and $\mathbf{2}$, and its seemingly random $\mathrm{Sn}-\mathrm{O}_{2} \mathrm{CCF}_{3}$ bond lengths suggested the ligands in $\mathbf{3}$ were labile in the condensed phase. Nevertheless, by comparing the five shortest $\mathrm{Sn}-\mathrm{O}$ distances in 3, we were able to group the $\mathrm{Sn}-\mathrm{O}$ bonds into three types: "weak" above average length bonds, "strong" below average length bonds, and short $\mu_{3}$-oxy bonds. These bond definitions ultimately revealed the fate of the structural elements of $\mathbf{3}$ when evaporating into $\mathbf{1}$ and $\mathbf{2}$ (Figure 6.9). 


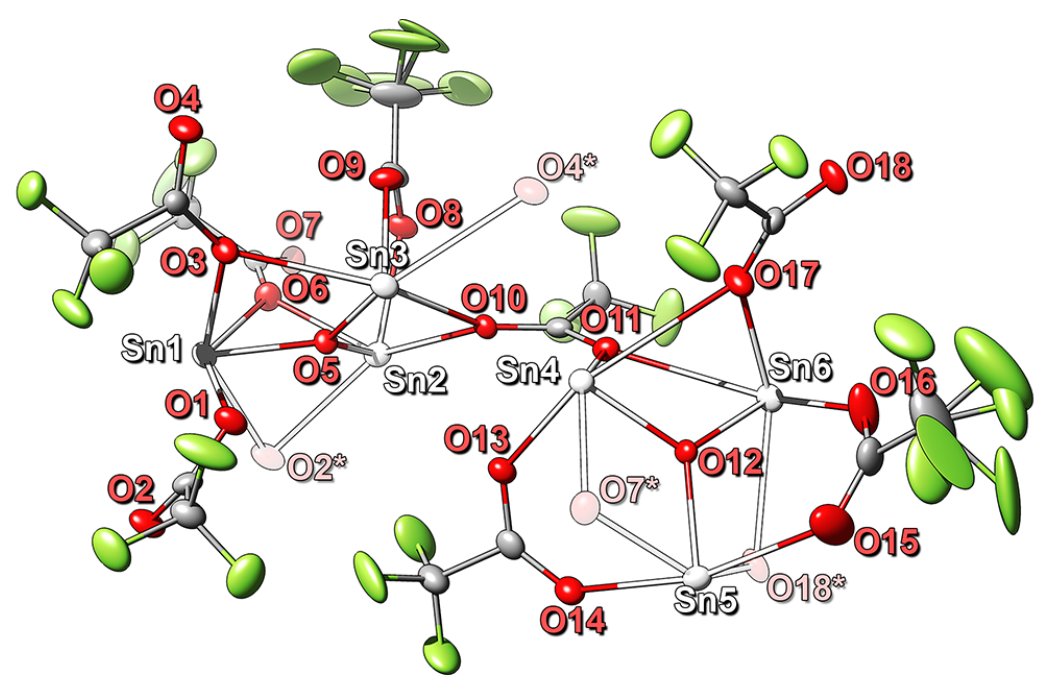

Figure 6.3: Asymmetric monomer of hexatin(II) di- $\mu_{3}$-oxy-octakis( $\mu$-trifluoroacetate) (3). Ellipsoids are set to $50 \%$ probability, disordered $\mathrm{CF}_{3}$ groups are included, and intermolecular contacts are drawn translucent

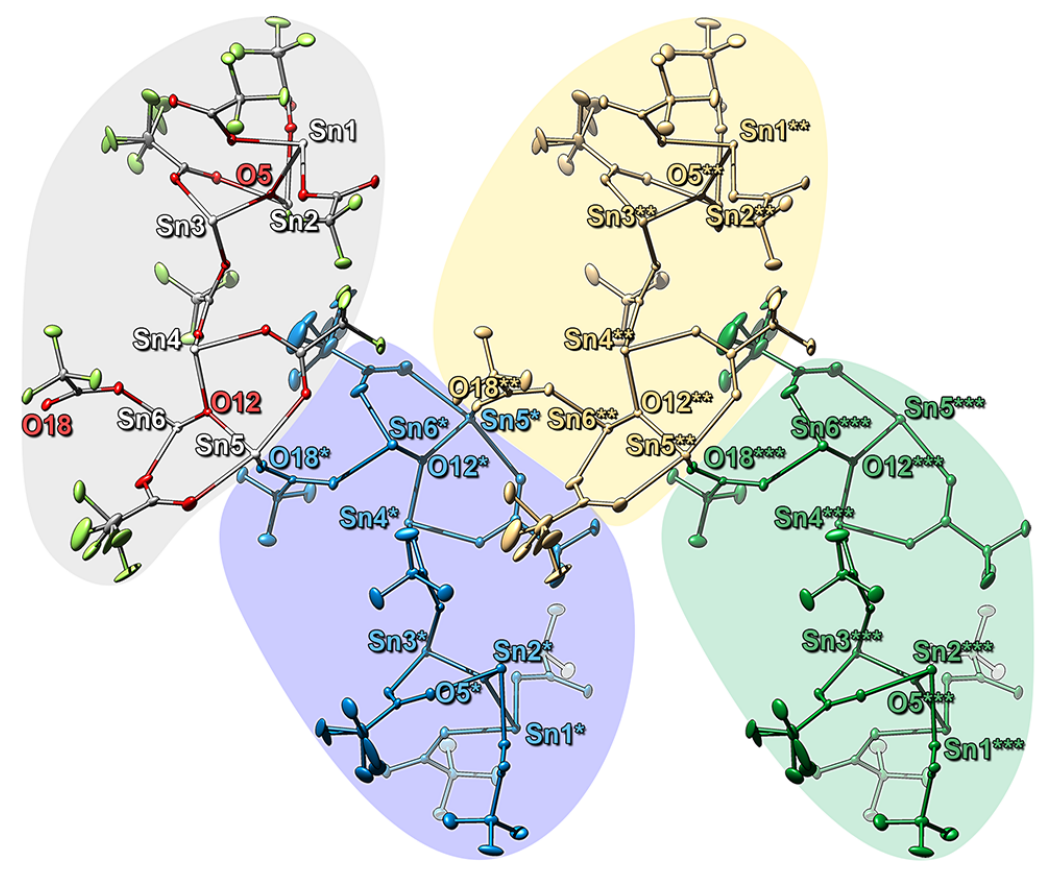

Figure 6.4: Polymeric chain of $(3)_{4}$. The first monomer (leftmost, gray highlight) is shown in Figure 6.1 (colored by element), while the other monomers are uniformly colored and highlighted (blue, yellow, and green from left to right) for clarity. Monomers are covalently bound through Sn5-O18* bonds. 


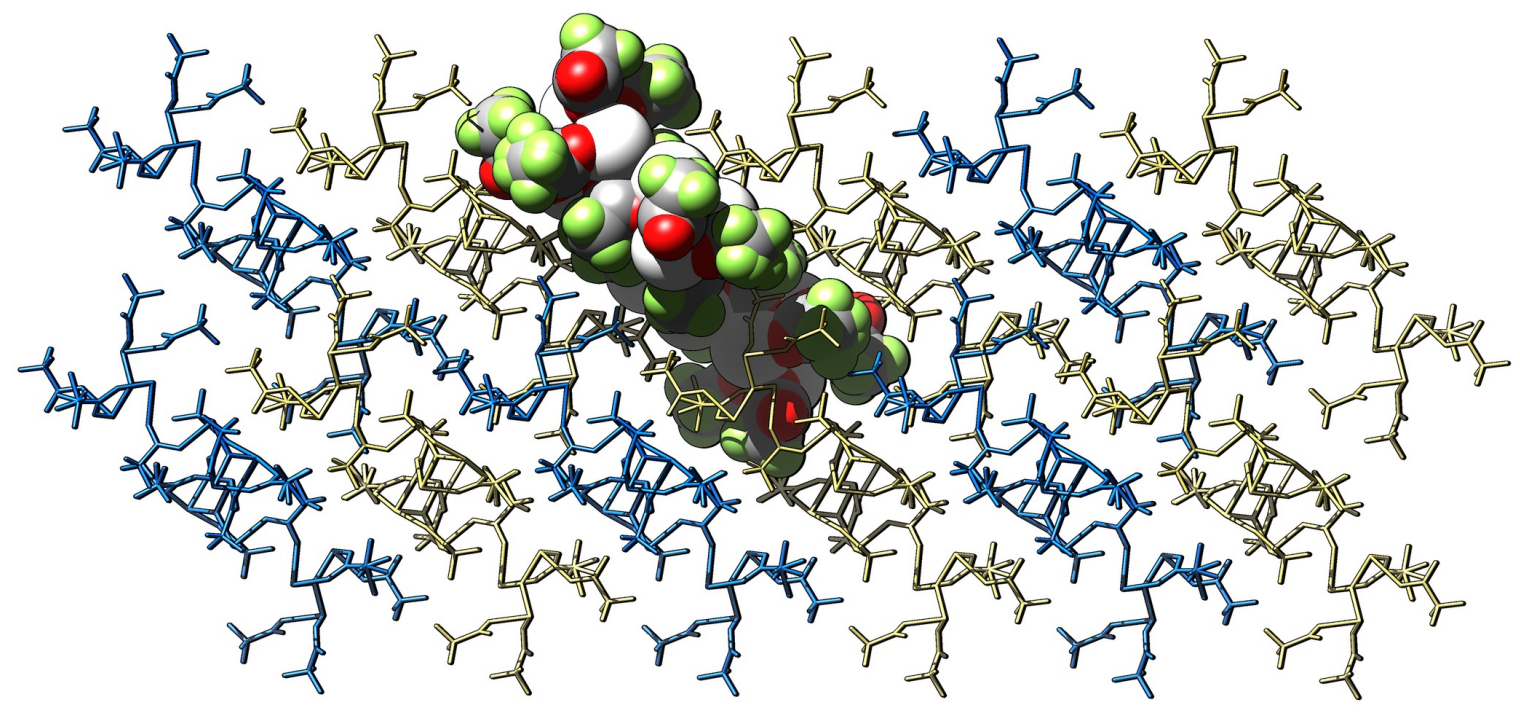

Figure 6.5: Crystal packing of a $3 \times 3 \times 3$ supercell of 3 . Chains bonded via $\mathrm{Sn}_{5}-\mathrm{O}_{18}{ }^{*}$ bonds are depicted as single-colour stick diagrams (blue or gold), except for one that is displayed as a spacefilling diagram with the elements coloured as in Figure 6.1. Fragments of chains surrounding the supercell have been omitted for clarity.

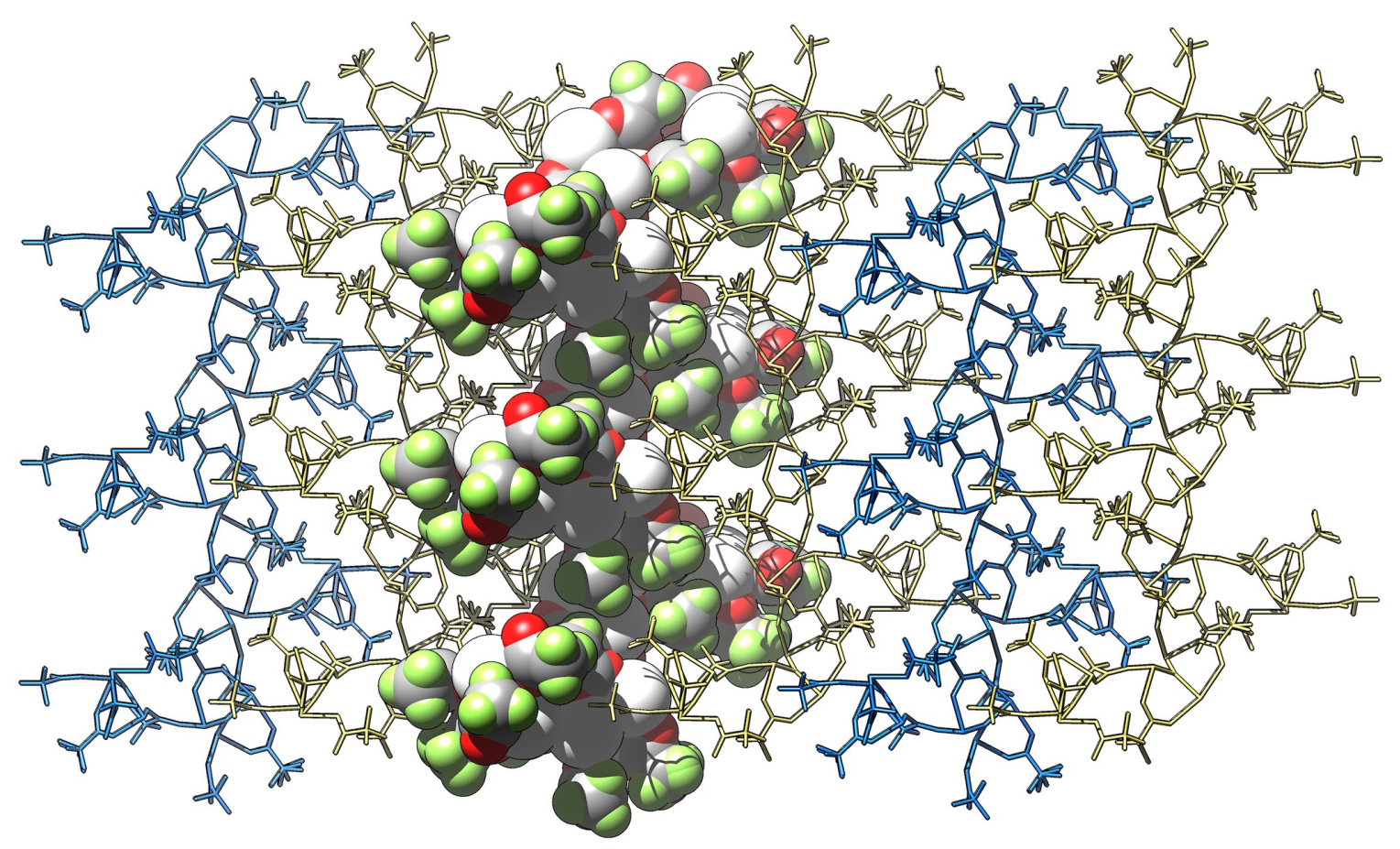

Figure 6.6: Crystal packing of a $3 \times 3 \times 3$ supercell of 3, viewed from the top of Figure 6.5. 


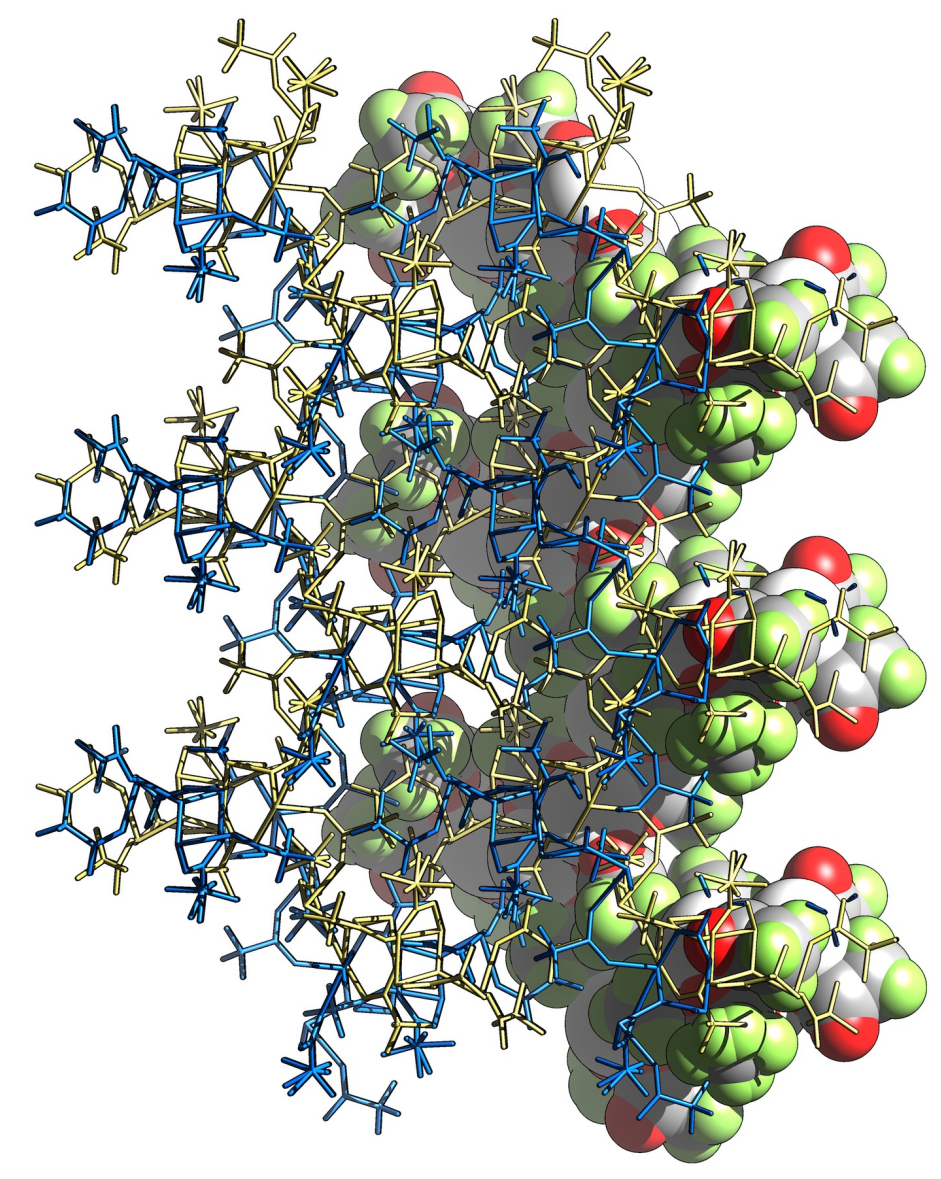

Figure 6.7: Crystal packing of a $3 \times 3 \times 3$ supercell of 3, viewed from the right of Figure 6.5.
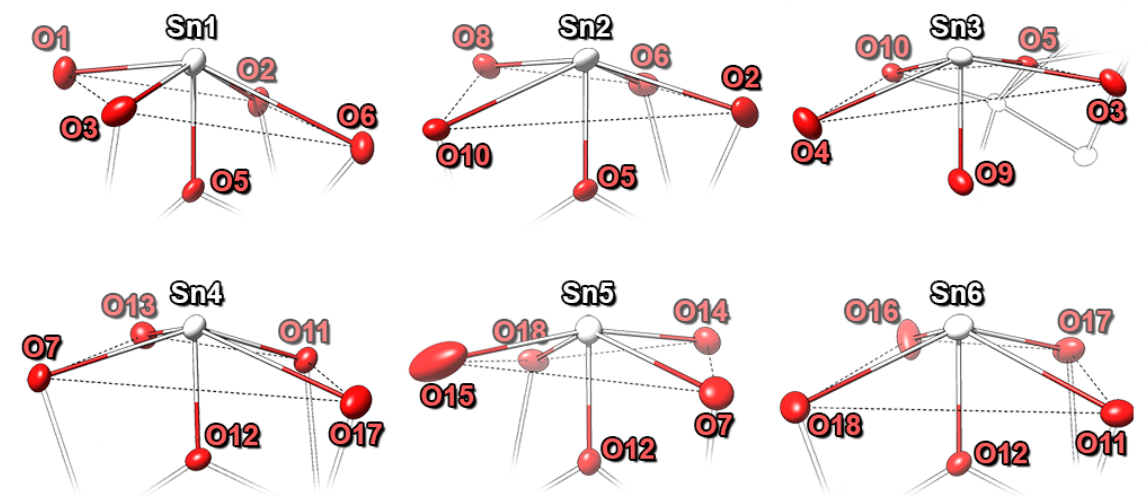

Figure 6.8: Extremely distorted pseudosquare pyramidal geometry around each $\mathrm{Sn}$ atom in 3 . Only the five shortest $\mathrm{Sn}-\mathrm{O}$ interactions are shown. 
Each $\mathrm{Sn}$ atom in 3 was bound to trigonal planar $\mu_{3}$-bridging oxygen atoms with similar bond lengths (ca. 2.0 $\AA$ with a $0.2 \AA$ deviation of $\mu_{3}-\mathrm{O}$ from $\mathrm{Sn}-\mathrm{Sn}-\mathrm{Sn}$ plane). This was reminiscent of the recently prepared "trapped" SnO complex. ${ }^{374}$ Natural bond order (NBO) analysis previously showed the trapped oxygen to have a strongly ionic character with three $\sigma$-bonds from lone pairs on the oxygen to empty orbitals on the three Sn atoms. Despite similarities, it is misleading to describe the $\mu_{3}$-oxygen in $\mathbf{3}$ as representing a "trapped" SnO species: one of the three $\mathrm{Sn}-\mu_{3}-\mathrm{O}$ bonds was shorter than the other two (i.e., Sn3 and Sn6), opposite to what was expected for trapped SnO donating to two other Sn atoms. This suggested that two $\mathrm{Sn}$ atoms in the $\mathrm{Sn}_{3}-\mu_{3}-\mathrm{O}$ group formed Lewis basic covalently bound $\mathrm{Sn}-\mathrm{O}-\mathrm{Sn}$ bridges, and the other accepted $\sigma$-electron density as a Lewis acid. Frontier orbital analysis of $\mathbf{1}$ and $\mathbf{2}$ supported this description, implying $\mathbf{3}$ was the polymeric Lewis pair $(\mathbf{1} \leftarrow 2)_{\infty}$ (Figure 6.9). This was an important distinction since it also predicted that the polymer should break into these Lewis acid/base units upon evaporation, as observed by GED. The orbital energies and distributions in the $\mathbf{1}$ and $\mathbf{2}$ fragments supported their roles as acid and base, respectively. Only the basic $\mathrm{Sn}-\mathrm{O}-\mathrm{Sn}$ bridge in 2 and complementary acidic orbitals in $\mathbf{1}$ had the appropriate symmetry to allow overlap to form Lewis pairs (Figure 6.10).

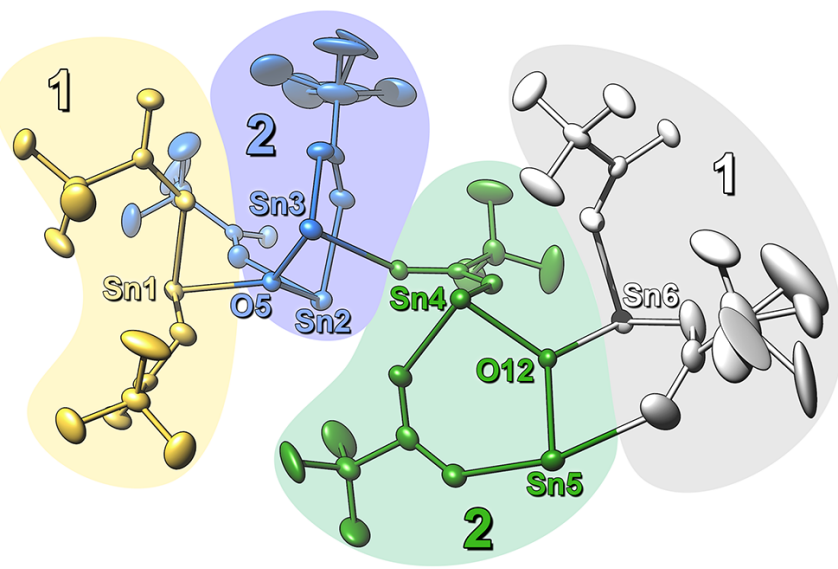

Figure 6.9: Asymmetric unit of 3 showing the fate upon evaporation of Sn 1 and Sn6 as 1 (gold, white; left and right) and $\operatorname{Sn}[2-3]$ and $\operatorname{Sn}[4-5]$ as 2 (blue, green; middle). 
Our difficulty growing crystals of $\mathbf{3}$ was not surprising. Previous reports of attempts to grow single crystals of $\mathbf{1}$ produced species like those we isolated. Compound $\mathbf{3}$ ligated with free trifluoroacetic acid was previously grown by heating $\mathbf{1}$ in a sealed evacuated tube, where protons from hydroxyl groups on the tube's walls were presumably abstracted by trifluoroacetic anhydride to form the acid ligand. ${ }^{367}$ The hydrated cluster compound tetratin(II) monotin(IV) di- $\mu_{3}$-oxy-octakis- $\mu$-trifluoroacetate (4) was previously grown by oxidation in $\mathrm{O}\left(\mathrm{OCCF}_{3}\right)_{2} / \mathrm{HO}_{2} \mathrm{CCF}_{3} ;{ }^{366}$ while we found crystals of anhydrous 4 near the seal of a pressure vessel after sublimation of pure 3 (Figure 6.13), presumably formed by oxidation with air that diffused through a degraded O-ring seal. Both of these previously reported compounds share structural motifs with 2 , highlighting the rich coordination chemistry in this tin-oxy-trifluoroacetate system.

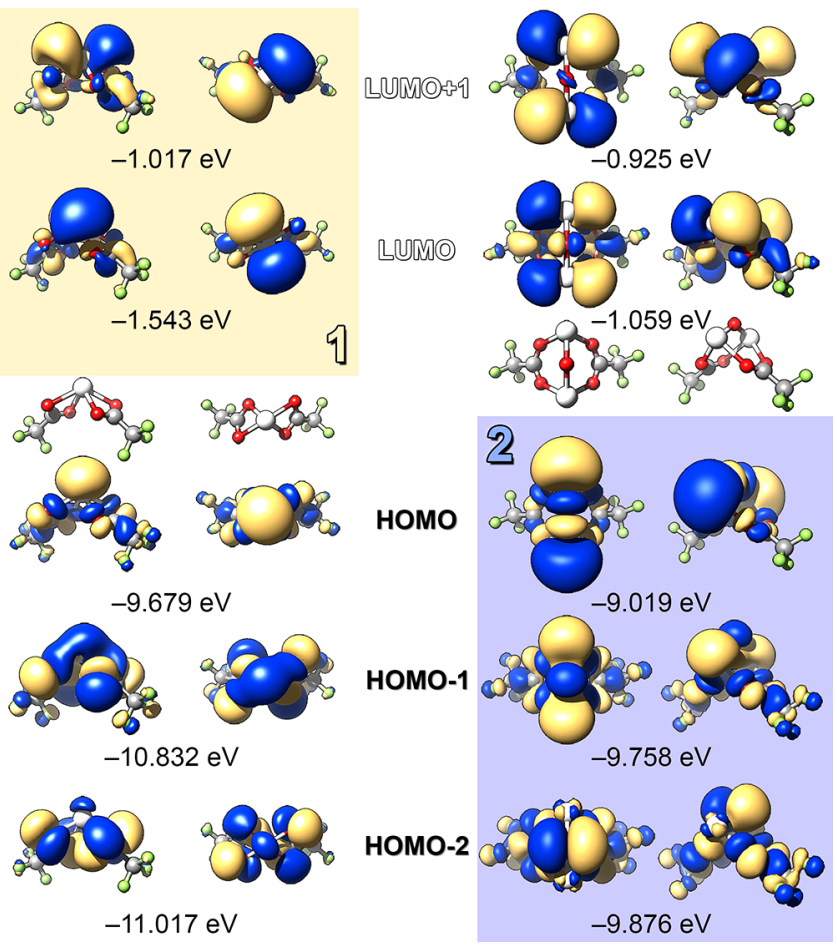

Figure 6.10: Frontier orbitals of $\mathbf{1}$ (left) and $\mathbf{2}$ (right), calculated by DFT (SOGGA11-X/DKH-TZP), viewed from the top (inside) and sides (outside). Lewis acidic and basic orbitals with the correct orbital symmetry overlap are highlighted in gold and blue, respectively 


\subsubsection{Tin(IV) Tetrakis(trifluoroacetate)}

Tin(IV) tetrakis(trifluoroacetate) (5) was also polymeric in the solid state, and yet was surprisingly volatile. A single crystal suitable for X-ray crystallography was grown by sublimation under nitrogen in a gently heated sealed tube $\left(\right.$ ca. $\left.50{ }^{\circ} \mathrm{C}\right)$. The structure of $\mathbf{5}$ consisted of symmetrical,

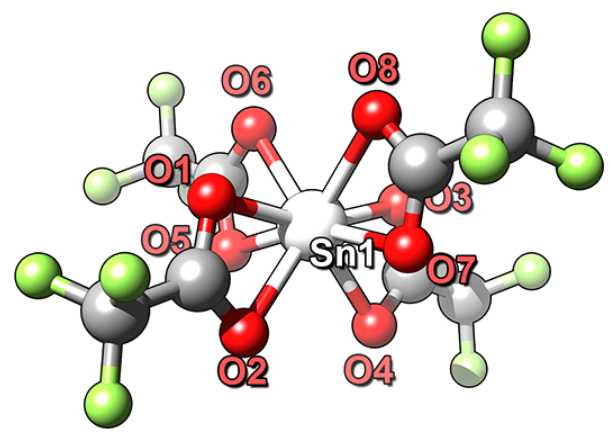
linear, non-interacting polymeric chains of octahedral Sn(IV) with four equatorial $\mu-$

Figure 6.11: Gas-phase structure of tin(IV) tetrakis(trifluoroacetate) (6). bridging and two monodentate axial ligands (Figure 6.12). Unlike 3, compound 5 was very sensitive to air and moisture, and its preparation and handling were more difficult. But its combination of high volatility and reactivity, along with a lack of $\mathrm{Sn}-\mathrm{C}$ bonds gives 5 the potential to be a near-room temperature precursor for $\mathrm{SnO}_{2}$ that may bypass current issues of low growth rate and high carbon contamination from other precursors. ${ }^{375,376}$ GED analysis showed that 5 evolves the expected monomer $\mathbf{6}$ with pseudo-dodecahedral coordination of four $\mathrm{K}_{2}$-trifluoroacetates upon evaporation (Figure 6.11). As with compound $\mathbf{3}$, the bulk composition of $\mathbf{5}$ was supported by the high quality of our refined GED data.

The solid state structures of $\mathbf{3}$ and $\mathbf{5}$ were different from their hydrogenated cousins tin(II) diacetate (7) and tin(IV) tetraacetate (8). Both 3 and 7 are linear polymers via "strong" Sn-O bonds (vide supra), where each Sn atom is tetracoordinate with Sn-OAc bonds in the range 2.17-2.37 $\AA .{ }^{377}$ However, 3 displays interchain coordination via bridging trifluoroacetate ligands while 7 forms tetrameric columns of (7)n chains with their stereochemically active lone pairs oriented inward and acetate ligands outward. This organization was explained as a steric effect, but the possibility of $\mathrm{CH}_{3}-\mathrm{O}$ interactions could 


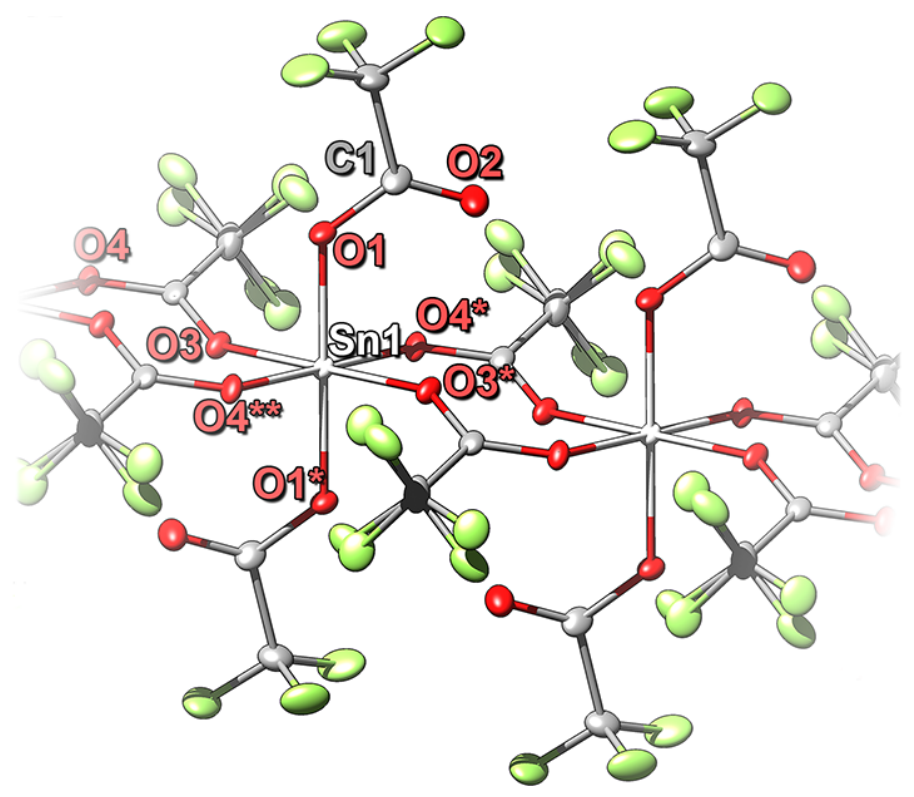

Figure 6.12: Solid state structure of polymeric 5. Disordered $\mathrm{CF}_{3}$ groups are shown, and ellipsoids are displayed at $50 \%$ probability.

not be ruled out. ${ }^{377}$ Compounds 1 and 7 are isostructural in the gas phase with C2 symmetry, showing very similar Sn-O bond lengths [2.186(22) and 2.395(25) vs. 2.192(8) and 2.337(12) A, respectively]. ${ }^{378}$ This is different from the gas-phase structure of 2 , and there is no precedent in the literature of compounds with an unligated $\mathrm{Sn}(\mathrm{II})-\mathrm{O}-\mathrm{Sn}$ (II) bridge, making 2 the first structurally characterized example. Comparison of $\mathbf{6}$, the gas phase monomer of $\mathbf{5}$, with the solid state structure of $\mathbf{8}$ show both are pseudo-dodecahedral with four bidentate ligands and similar Sn-O bond lengths in the range 2.13-2.29 $\AA .{ }^{379}$

\subsubsection{Evaporation Kinetics and Self-Limiting Behaviour}

Thermogravimetric analysis (TGA) of hexatin(II) di- $\mu_{3}$-oxy-octakis- $\mu$-trifluoroacetate (3) and tin(IV) tetrakis(trifluoroacetate) $\mathbf{5}$ showed their excellent thermal stability and volatility compared to tin(II) diacetate (7) and tin(IV) tetraacetate (8) (Figures 6.13 and 6.14). Both $\mathbf{3}$ and $\mathbf{5}$ had single step volatilization with no decomposition, which was ideal behaviour for potential ALD precursors. Decomposition during TGA can be evaluated simply by increasing the initial mass loading, in what is called a "thermal stress" test. ${ }^{85}$ Simply, a 


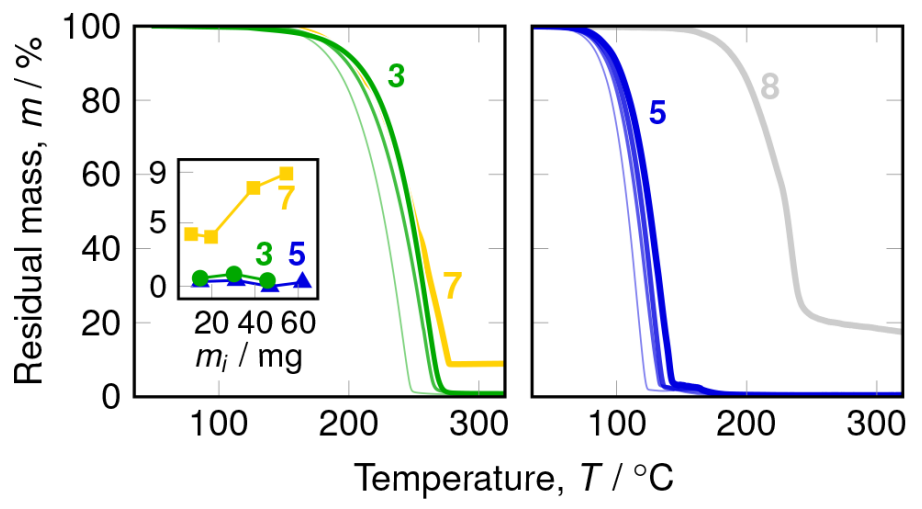

Figure 6.13: Ramped thermogravimetric analysis (TGA) curves of hexatin(II) di- $\mu_{3}$-oxy-octakis$\mu$-trifluoroacetate (3, left, green), tin(II) diacetate (7, left, gold), tin(IV) tetrakis(trifluoroacetate) (5, right, blue) and tin(IV) tetraacetate (8, right, gray). Stress tests (inset) reveal the stability of $\mathbf{3}$ (green circles) and $\mathbf{5}$ (blue triangles) with no increase in residual mass with increasing initial mass loading; and highlight the instability of $\mathbf{7}$ (gold squares) with a positive correlation. 8 was omitted as it decomposed considerably even at low mass loading (ca. $10 \mathrm{mg}$ ), so its comparison becomes trivial.

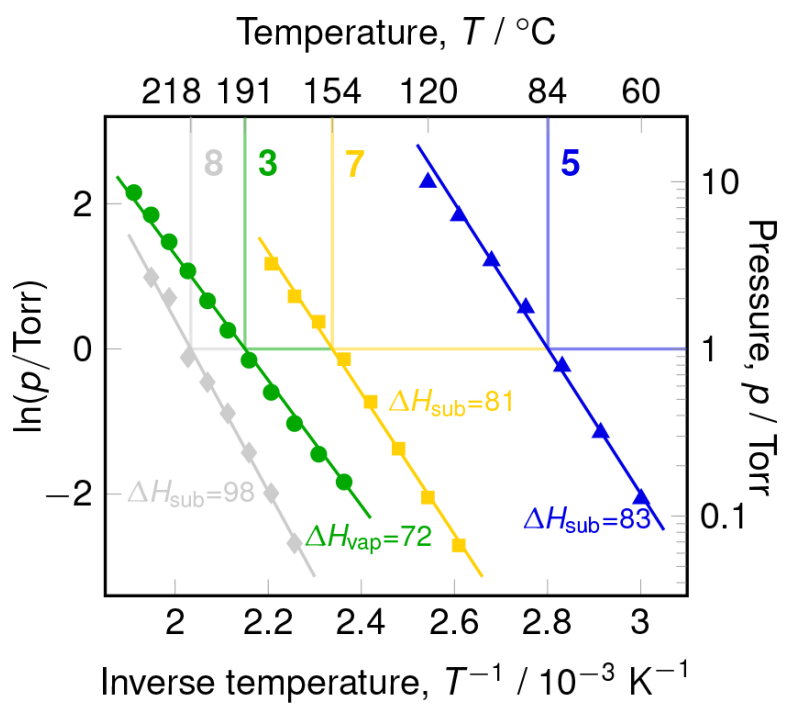

Figure 6.14: Vapour pressure of $\mathbf{3}$ (green circles), $\mathbf{7}$ (gold squares), $\mathbf{2}$ (blue triangles), and $\mathbf{8}$ (gray diamonds) as estimated by thermogravimetric analysis. Enthalpies (in $\mathrm{kJ} / \mathrm{mol}$ ) of evaporation (for 3 ) and sublimation $(2,7$, and 8$)$ were estimated by the Clausius-Clapeyron equation. 
higher loading of analyte requires more time to evaporate, so a higher mass of analyte is exposed to higher temperatures for longer time. Thus, higher temperature decomposition events may occur and be observed during an experiment as an increase in non-volatile residual mass. This stress test can be used to qualitatively evaluate the onset of decomposition and overall stability of a potential precursor. ${ }^{85,182}$ While 7 decomposed to $\mathrm{SnO}$ at $238{ }^{\circ} \mathrm{C},{ }^{380} 3$ shows negligible decomposition up to at least $275{ }^{\circ} \mathrm{C}$ by TGA, at which temperature the analyte had completely evaporated.

Despite its relatively lower volatility, 3 performed exceptionally well under realistic ALD process conditions, surviving over a week of continuous heating at $170{ }^{\circ} \mathrm{C}$ with no change in its thermal properties. We were able to reproduce the previously reported promising thermal behavior of $1:^{363}$ no films were deposited at $400{ }^{\circ} \mathrm{C}$ in a commercial ALD reactor after $2000 \times 8 \mathrm{~s}$ pulses without an oxidizing co-reactant. This strongly suggests 1 and 2 can form a stable monolayer that is critical to ALD growth. The ease of synthesis and handling, high thermal stability, and good volatility make 3 a promising ALD precursor for F-doped $\mathrm{SnO}_{2}$.

The dramatic difference in volatility among this family of compounds could not be explained by their enthalpies of vaporization: the temperature at which they reached 1 Torr of vapour pressure decreased from $8\left(218{ }^{\circ} \mathrm{C}\right), 3\left(191^{\circ} \mathrm{C}\right), 7\left(154^{\circ} \mathrm{C}\right)$, to $5\left(84^{\circ} \mathrm{C}\right)$, while their $\Delta H_{\mathrm{vap}}$ increased in the order $3\left(72 \mathrm{~kJ} \mathrm{~mol}^{-1}\right), 7\left(81 \mathrm{~kJ} \mathrm{~mol}^{-1}\right), \mathbf{5}\left(83 \mathrm{~kJ} \mathrm{~mol}^{-1}\right), 8\left(98 \mathrm{~kJ} \mathrm{~mol}^{-1}\right)$. One would expect 8, a coordinately saturated complex of moderate molecular weight, to be more volatile than the polymeric compounds. But it was the least volatile by a large margin, reaching a pressure of $\mathbf{1}$ Torr at $134{ }^{\circ} \mathrm{C}$ higher than 5. It is also surprising that 3 has the smallest $\Delta H_{\mathrm{vap}}$ even though many $\mathrm{Sn}-\mathrm{O}$ coordinative bonds must be broken to evaporate as $\mathbf{1}$ and 2 .

Of course, the Gibbs free energy of evaporation depends on the change in entropy $\Delta S$ as well as change in enthalpy $\Delta H$, and the change in entropy of a system from a condensed phase to the vapour phase is related to the number of possible microstates $W$ available 
in that phase by the Boltzmann equation. Since vaporization is an endothermic process where $\Delta G \leq 0, \Delta H$ and $\Delta S$ must then both be positive. For highly symmetrical polymers like $5, \Delta S$ is large due to the depolymerization from one $(5)_{n}$ polymer into $n$ gas phase monomers (naively, $W_{g} \approx n W_{c}$, where $W_{c}$ represents the condensed phase, and $W_{g}$ the gas phase), lowering the temperature required to satisfy $\Delta H \leq T \Delta S$. A similar argument can be made for the symmetrical polymer $7^{358,377,378}$ but its lower vapour pressure is likely due to the secondary factor of increased intermolecular interactions between more polarizable $-\mathrm{CH}_{3}$ groups when compared to $-\mathrm{CF}_{3} .{ }^{377}$ The complexity and lability of $\mathbf{3}$ in the condensed phase lessens the difference between $W_{g}$ and $W_{c}$, increasing the temperature required to satisfy the Gibbs inequality, decreasing its volatility. Similarly, the change in entropy upon vaporization of $\mathbf{8}$ is small due to its monomeric nature in the solid state ${ }^{379}$ and gas phase. Overall, this suggests a new strategy in precursor design: volatility can be increased by forming highly symmetrical coordination polymers to exploit their inherently large change in entropy upon evaporation into monomers.

\subsubsection{Supramolecular Organization}

The variability in polymerization we found in 3 suggested that addition of additional Lewis bases could easily disrupt its structure. Exploiting this, we structurally characterized several other compounds and uncovered some patterns of tin trifluoroacetate supramolecular organization: the

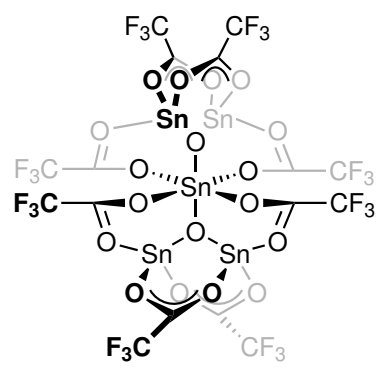

4

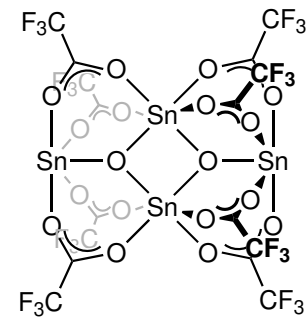

9 cage-like cluster is[tin(II) bis(trifluoroacetate) trimethylphosphine] $\left[\left(1 \leftarrow \mathrm{PMe}_{3}\right)_{6}\right]$; the anhydrous partial oxidation product of $\mathbf{3}$, compound $4 ;{ }^{366}$ and the diethyl ether $\sigma$-adduct $\mathbf{6} \leftarrow\left(\mathrm{OEt}_{2}\right)_{2}$ (Figure 6.13). These are also similar to the previously reported mixed valence cluster ditin(II) ditin(IV) di- $\mu_{3}$-oxy-octakis- $\mu$-trifluoroacetate (9). ${ }^{369} \mathrm{With}$ these pieces we 
can complete the picture of supramolecular organization in the tin-oxy-trifluoroacetate system: $\operatorname{Sn}(\mathrm{II})$ centers adopt distorted square pyramidal five-coordinate geometries. $\mathrm{Sn}(\mathrm{IV})$ centers adopt octahedral geometries which distort upon coordination to $\mathrm{Sn}(\mathrm{II})$ atoms. Homoleptic trifluoroacetates naturally act as Lewis acids, while trifluoroacetate groups bridge rather than chelate Sn centers to fill their coordination spheres, and bond more strongly to $\mathrm{Sn}(\mathrm{IV})$ (bond lengths ca. $2.1 \AA$ ) than the more electron-rich $\mathrm{Sn}$ (II) centers (bond lengths $>2.3 \AA$ ). Trifluoroacetic anhydride is apparently readily eliminated, ${ }^{368,381,382}$ leaving oxy moeties that donate $\sigma$-electron density to form basic $\mathrm{Sn}-\mathrm{O}-\mathrm{Sn}$ bridges and thus $\mu_{3}$-oxy bridged Lewis pairs.

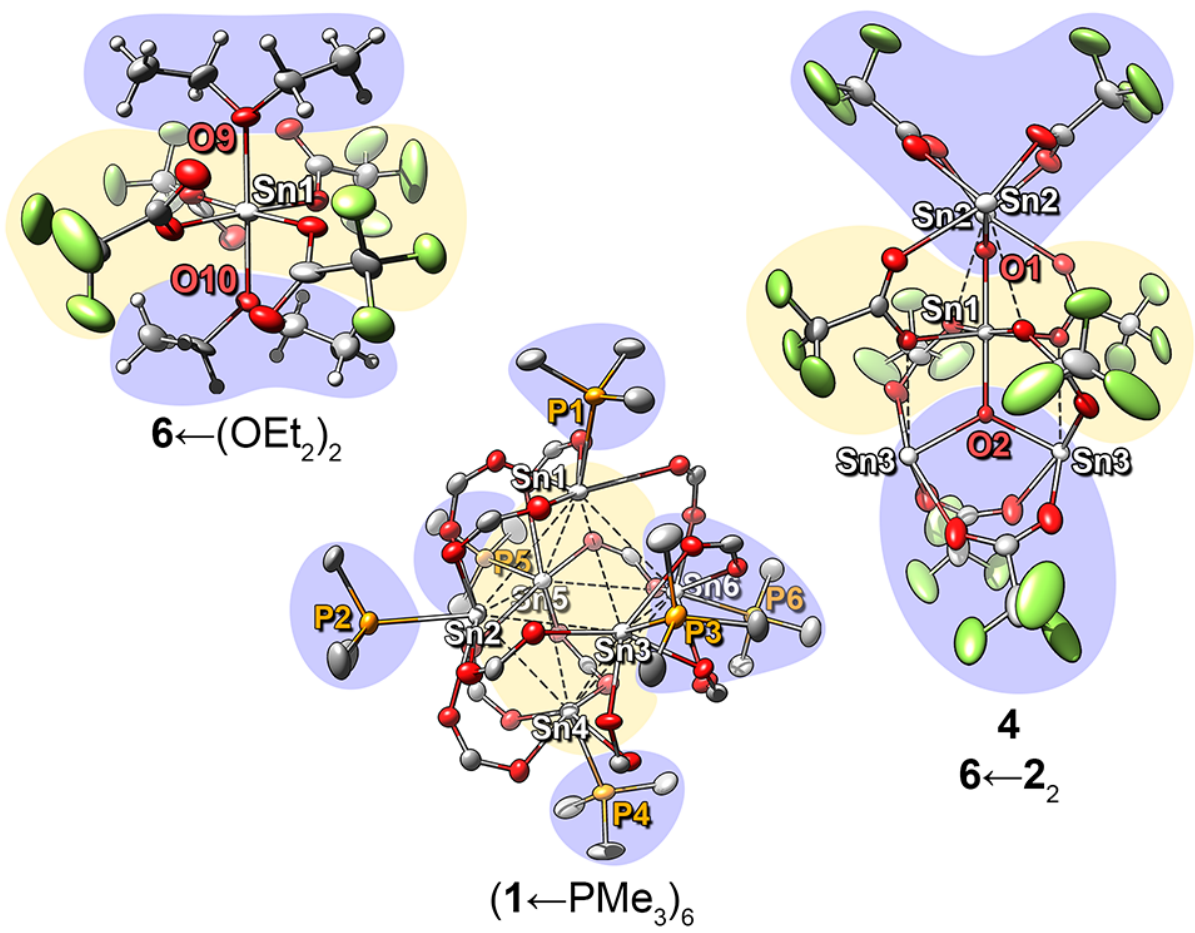

Figure 6.15: Molecular structures $6 \leftarrow\left(\mathrm{OEt}_{2}\right)_{2}$ (left), $\left(\mathbf{1} \leftarrow \mathrm{PMe}_{3}\right)_{6}$ (center), and 4 (right). Lewis basic groups (i.e., $\mathrm{Et}_{2} \mathrm{O}, 2$, and $\mathrm{PMe}_{3}$ ) are highlighted in blue, and Lewis acidic groups (i.e., $\mathbf{1}$ and 6) are highlighted in gold to show how 4 can be described as $6 \leftarrow(2)_{2}$. Ellipsoids displayed at $50 \%$ probability. $\mathrm{CF}_{3}$ groups and hydrogens are omitted from $\left(1 \leftarrow \mathrm{PMe}_{3}\right)_{6}$ for clarity.

Together, 1, 2, 6, and the inferred trifluoroacetic anhydride elimination product of $\mathbf{6}$ "OSn $\left(\mathrm{O}_{2} \mathrm{CCF}_{3}\right)_{2}$ " (10) make up the elementary building blocks of tin-oxy-trifluoroacetate clusters and polymers. Consequently, 3 is more appropriately described as $(\mathbf{1} \leftarrow 2)_{\infty}$, com- 
pound 4 as $\mathbf{6} \leftarrow(2)_{2}$, and compound 9 as $(\mathbf{1} \leftarrow \mathbf{1 0})_{2}$. It is notable that $\mathbf{6}$ and $\mathbf{1 0}$ do not form a stable co-subliming complex like $\mathbf{1}$ and $\mathbf{2}$ do in $\mathbf{3}$. If $\mathbf{9}$ is a typical example and $\mathbf{1 0}$ dimerizes like the central four-membered $\mathrm{Sn}_{2} \mathrm{O}_{2}$ heterocycle, then it is likely that intimate coordination between $\mathbf{6}$ and $\mathbf{1 0}$ is prevented by steric hinderance. The preparation and isolation of free $\mathbf{2}$ and $\mathbf{1 0}$ has not yet been successful, but the combination of these building blocks and other Lewis acids and bases opens a potentially versatile toolbox of thermally robust and amphoteric tin-containing supramolecular complexes.

\subsection{Conclusions}

Contrary to conventional wisdom, our results demonstrate that polymerization does not need to be avoided in precursor design if reversible thermal depolymerization can be achieved. In fact, it may increase the volatility over monomeric analogues. Despite its relatively lower volatility; the low cost, safe handling, and easy preparation of $\mathbf{3}$ make it the more attractive F-doped $\mathrm{SnO}_{2}$ precursor for many applications. Compound 5 has potential as a complementary precursor for specialized application in very low temperature deposition. We are currently exploring processes using $\mathbf{3}$ or $\mathbf{5}$ as precursors to F-doped $\mathrm{SnO}_{2}$ by ALD.

\subsection{Experimental Details}

\subsubsection{Synthetic Details}

\subsubsection{General Synthetic Considerations}

Most of the preparations reported below are oxygen- and moisture-sensitive, so they were performed under an inert atmosphere in a nitrogen-filled ("4.8" 99.998\% purity) MBraun Labmaster 130 dry-box or under ultrapure nitrogen gas ("5.0" 99.999\% purity) using standard Schlenk techniques. Those that can be performed under ambient conditions are noted 
as such. Low vacuum was achieved by a digitally regulated diaphragm pump (Buchi ${ }^{\circledR}$ VacoboxTM, min. ca. 10 Torr), and high vacuum by single-stage rotary vane pumps (Edwards RV5) whose absolute base pressures were measured to be 1-10 mTorr by a Hgfilled McLeod gauge. All solvents (e.g., toluene, hexanes, diethyl ether) were ACS Reagent grade or higher, degassed by an MBraun Solvent Purifier System using nitrogen gas ("4.8" 99.998\% purity), and stored in a dry-box over freshly-regenerated $4 \AA$ molecular sieves (Millipore-Sigma) for at least $24 \mathrm{~h}$ before use. Deuterated solvents were either used from a freshly opened ampoule, or degassed by three freeze-pump-thaw cycles then dried and stored over molecular sieves. Reverse osmosis water was deionized using a MilliQ system until its resistance reached $18 \mathrm{M}$. All purchased chemicals were used as received. Tin(II) oxide ( $\geq 95 \%$ ), copper (II) oxide (powder, $<10 \mu \mathrm{m}, 98 \%$ ), silver(I) oxide (ReagentPlus ${ }^{\circledR}, 99 \%$ ), trifluoroacetic acid (ReagentPlus ${ }^{\circledR}, 99 \%$ ), trifluoroacetic anhydride (ReagentPlus ${ }^{\circledR}, \geq 99 \%$ ), and tin(IV) bromide (99\%) were purchased from Millipore-Sigma. Tin metal (powder, 100 mesh, 99.5\%) was purchased from Strem Chemicals. Tin(II) diacetate (7), ${ }^{380} \operatorname{tin}(\mathrm{IV})$ tetraacetate (8), ${ }^{383}$ silver(I) trifluoroacetate, ${ }^{384}$ and copper(II) bis(2,2,6,6-tetramethylheptane-3,5dionate) $\left[\mathrm{Cu}(\mathrm{tmhd})_{2}\right]^{385}$ were prepared according to literature methods. NMR spectra were collected with a Bruker Avance II $300 \mathrm{MHz}$ spectrometer at room temperature.

\subsubsection{Hexatin(II) di- $\mu_{3}$-oxy-octakis- $\mu$-trifluoroacetate (3)}

Caution! During experiments using trifluoroacetic acid, all ground glass fittings were sealed with PTFE thread. Silicone vacuum grease is very soluble in trifluoroacetic acid: prolonged reflux will lead to frozen joints and leaks, and filtration by inversion through a filter stick will allow trifluoroacetic acid to pass unimpeded through the glass joint, posing a serious safety risk due to acid burns from exposure to the vapour or liquid.

By oxidation of $\mathrm{Sn}(0)$ with $\mathrm{Cu}\left(\mathrm{O}_{2} \mathrm{CCF}_{3}\right)_{2}$ : Copper(II) bis(trifluoroacetate) was prepared in situ according to a modified literature method under ambient conditions. ${ }^{386}$ One equivalent of copper(II) oxide $(9.325 \mathrm{~g}, 116.0 \mathrm{mmol})$ was suspended in $200 \mathrm{~mL}$ water in a $500 \mathrm{~mL}$ 
round bottom flask with vigorous magnetic stirring. Two equivalents of trifluoroacetic acid (26.459 g, $232.1 \mathrm{mmol})$ was added in a continuous stream through an addition funnel. An exotherm occurred and the mixture was stirred with gentle $\left(40{ }^{\circ} \mathrm{C}\right.$, overnight $)$ or no heating (3 days) until it became azure blue and mostly transparent. The mixture was then filtered with suction through a Celite pad and a medium porosity fritted glass filter to remove unreacted $\mathrm{CuO}$ and other insoluble impurities to yield a clear azure blue solution. This was then deoxygenated by sparging for $30 \mathrm{~min}$ with a vigorous nitrogen flow through a fritted glass gas-diffusion tube.

Then, an excess of tin metal powder $(27.548,232.1 \mathrm{mmol})$ was added all at once to the aqueous $\mathrm{Cu}\left(\mathrm{O}_{2} \mathrm{CCF}_{3}\right)_{2}$ solution under a stream of nitrogen gas at ambient temperature. Copper metal was clearly visible after an hour as shiny flakes, which were obscured after a day by re-deposition of excess tin. Completion of the reaction was indicated by a fast settling, dark grey precipitate ( $\mathrm{Cu}$ plated with $\mathrm{Sn})$ and a colourless supernatant liquid, which was separated from the precipitate by filtration through a fritted glass filter stick under nitrogen to yield a colourless solution. Volatiles were removed from the filtrate first by heating under low vacuum (ca. 10 Torr, $60{ }^{\circ} \mathrm{C}, 4 \mathrm{~h}$ ) to yield a pale-yellow syrup that eventually becomes a colourless solid. This was dried with heating under high vacuum (1-10 mTorr, $100{ }^{\circ} \mathrm{C}, 8 \mathrm{~h}$ ) to yield $27.753 \mathrm{~g}$ of a colourless crystalline solid ( $87 \%$ crude yield based on $\mathrm{CuO}$ and $\mathrm{HO}_{2} \mathrm{CCF}_{3}$ used, assuming conversion to $1 / 6$ eq. of 3). Sublimation by vacuum-transfer (1-10 mTorr, $\left.170{ }^{\circ} \mathrm{C}\right)$ into a cooled $\left(-78^{\circ} \mathrm{C}\right)$ receiving flask yielded $3(26.120 \mathrm{~g}, 82 \%$ yield $)$ as a colourless, sticky, amorphous solid that becomes a colourless crystalline solid that can be powdered upon cooling to $-45^{\circ} \mathrm{C}$ overnight. The title compound was deliquescent, and must be stored dry, although dry $\mathrm{O}_{2}$ does not appear to degrade samples. Oxidation occurs slowly and $\mathbf{3}$ can be briefly handled under ambient conditions. Complex $\mathbf{3}$ was very soluble in aprotic coordinating solvents (e.g., THF, ACN, DMSO), and slightly soluble in aromatic hydrocarbons (e.g., toluene). It forms a stable aqueous solutions in the absence of $\mathrm{O}_{2}$. M.p. $127^{\circ} \mathrm{C}$, sublimed. Crystals suitable for X-ray crystallography were grown by 
slow cooling of a toluene solution of $\mathbf{3}$ (previously sublimed twice). HRMS (EI+): Found $m / z=578.7624$, calculated $\mathrm{Sn}_{2}\left(\mathrm{O}_{2} \mathrm{CCF}_{3}\right)_{3}$ 578.7595, dev. $2.69 \mathrm{mmu} ; \mathrm{m} / z=481.7718$, calc. $\mathrm{Sn}_{2} \mathrm{O}\left(\mathrm{O}_{2} \mathrm{CCF}_{3}\right)_{2}$ 481.7694, dev. $2.40 \mathrm{mmu} ; m / z=364.7866$, calc. $\mathrm{Sn}_{2} \mathrm{O}\left(\mathrm{O}_{2} \mathrm{CCF}_{3}\right) 364.7867$, dev. $0.1 \mathrm{mmu} ; m / z=276.8633$, calc. $\mathrm{Sn}\left(\mathrm{O}_{2} \mathrm{CCF}_{3}\right) \mathrm{CO}_{2}$ 276.8771, dev. 13.8mmu. NMR chemical shifts of 3 in $\mathrm{D} 3 \mathrm{CCN}$ : $1 \mathrm{H}\left(\delta_{0}=1.94 \mathrm{ppm}\right)$ : silent; $13 \mathrm{C} 1 \mathrm{H}\left(\delta_{0}=1.32 \mathrm{ppm}\right): 117.03 \mathrm{ppm}$ $\left(q, \mathrm{O}_{2} \mathrm{CCF}_{3},{ }^{1} \mathcal{F}_{\mathrm{C}-\mathrm{F}}=288 \mathrm{~Hz}\right), 162.19 \mathrm{ppm}\left(q, \mathrm{O}_{2} \mathrm{CCF}_{3},{ }^{2} \mathcal{F}_{\mathrm{C}-\mathrm{F}}=38.7 \mathrm{~Hz}\right) ;{ }^{19} \mathrm{~F}$ (external reference, $\mathrm{HO}_{2} \mathrm{CCF}_{3}$ in $\mathrm{D}_{2} \mathrm{O} \delta_{0}=-75.15 \mathrm{ppm}$ ): $-75.61 \mathrm{ppm}(s) .{ }^{119} \mathrm{Sn}$ (external reference, $\mathrm{Sn}\left[\mathrm{N}\left(\mathrm{SiMe}_{3}\right)_{2}\right]_{2}$ in $\mathrm{C}_{6} \mathrm{D}_{6} \delta_{0}=770 \mathrm{ppm}$ ): silent. (Note: the combined effects of dynamic solvent exchange and compounded ${ }^{2} \mathcal{F}_{\mathrm{Sn}-\mathrm{C}},{ }^{2} \mathcal{F}_{119 \mathrm{Sn}-119 \mathrm{Sn},},{ }^{2} \mathcal{F}_{119 \mathrm{Sn}-117 \mathrm{Sn}}{ }^{2} \mathcal{F}_{119 \mathrm{Sn}-\mathrm{C}},{ }^{3} \mathcal{F}_{119 \mathrm{Sn}-\mathrm{C}}$, and ${ }^{4} \mathcal{F}_{119 S n-F}$ coupling likely led to extreme broadening of the signal to the point where it could not be detected.)

By condensation of $\mathrm{SnO}$ with trifluoroacetic acid: performed according to a modified literature procedure. ${ }^{366}$ Blue-black tin(II) oxide $(6.310 \mathrm{~g}, 46.84 \mathrm{mmol})$ was placed in a $250 \mathrm{~mL}$ Schlenk flask and dried under high vacuum (10 mTorr) at room temperature for $3 \mathrm{~h}$. The flask was refilled with nitrogen and fitted with a water-cooled reflux condenser, previously flamed-dried under vacuum, closed with a rubber sepctum. One equivalent of trifluoroacetic anhydride $(9.838 \mathrm{~g}, 46.84 \mathrm{mmol})$ in a large excess of trifluoroacetic acid $(100 \mathrm{~mL}$, $760 \mathrm{mmol}$ ) was deoxygenated by three freeze- pump-thaw cycles, then cannulated onto the $\mathrm{SnO}$ through the condenser. The mixture was heated to reflux with vigorous stirring under nitrogen for eight days, then cooled to room temperature to yield a colourless supernatant liquid and a sticky beige precipitate. This mixture was filtered through a fritted glass filter stick under nitrogen to yield a colourless solution. The filtrate was worked up and purified in a manner identical to the one detailed above to yield a colourless solid (11.676 g, 85\% yield assuming conversion to 3) that had identical thermal and spectroscopic characteristics to the material prepared by redox.

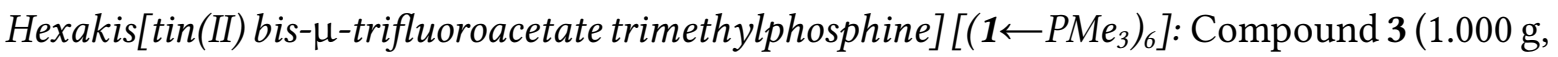
$0.6066 \mathrm{mmol})$ was suspended in toluene $(10 \mathrm{~mL})$ and trimethylphosphine $(0.221 \mathrm{~g}, 2.90 \mathrm{mmol}$, 
in $10 \mathrm{~mL}$ toluene) was added dropwise resulting in a clear colorless solution. $\left(\mathrm{PMe}_{3}\right.$ was added in excess due to our presumption that the starting material was $1, M=344.74 \mathrm{~g} / \mathrm{mol}$, before we discovered it was 3 .) Crystals of $\left(\mathbf{1} \leftarrow \mathrm{PMe}_{3}\right)_{6}$ were grown by slow cooling this solution to $-45^{\circ} \mathrm{C}$ for a week.

Tetratin(II) monotin(IV) di- $\mu_{3}$-oxy-octakis- $\mu$-trifluoroacetate (4): Crystals of 4 were grown by placing a sample of 3 (ca. $100 \mathrm{mg}$ ) in a PTFE-capped O-ring sealed glass pressure vessel in a dry-box and heating in an oven at $200{ }^{\circ} \mathrm{C}$ for $2 \mathrm{~h}$ until the sample had completely evaporated. Heating was stopped, and the oven was allowed to cool slowly. Large sticky crystals (presumably 3) were deposited throughout the vessel but could not be successfully harvested. Smaller cubic crystals of 4 suitable for crystallography were found near the cap of the vessel.

\subsubsection{Tin(IV) tetrakis(trifluoroacetate) (5)}

Tin(IV) tetrakis(trifluoroacetate) (5) was prepared according to a modified literature procedure. ${ }^{387}$ Tetraphenyltin $(4.271 \mathrm{~g}, 10.00 \mathrm{mmol})$ was placed in a $250 \mathrm{~mL}$ Schlenk flask, then degassed and dried under high vacuum at room temperature for one hour. One equivalent of trifluoroacetic anhydride $(2.100 \mathrm{~g}, 10.00 \mathrm{mmol})$ in a large excess of trifluoroacetic acid $(100 \mathrm{~mL})$ was cannulated into the flask. The suspension was stirred until it became a clear green/tan solution (about 1 day), then the volatiles were removed at ambient temperature under high vacuum. The resulting pale yellow/green solid was purified by vacuum transfer $\left(10\right.$ mTorr, $\left.60{ }^{\circ} \mathrm{C}\right)$ to yield a colourless crystalline solid contaminated with a less volatile by-product (observed by TGA) believed to be the product upon elimination of trifluoroacetic anhydride (i.e., "SnO( $\left.\mathrm{O}_{2} \mathrm{CCF}_{3}\right)_{2}$ ", 10). The solid was sublimed again (10 mTorr, $\left.60{ }^{\circ} \mathrm{C}\right)$ to yield $5\left(3.140\right.$ g, $55 \%$ based on $\mathrm{Ph}_{4} \mathrm{Sn}$, m.p. $115{ }^{\circ} \mathrm{C}$, lit. $\left.114-115{ }^{\circ} \mathrm{C} 38\right)$ as a colourless crystalline and very moisture-sensitive solid. Compound $\mathbf{5}$ is very soluble in trifluoroacetic acid, coordinating aprotic solvents, and moderately soluble in aromatic hydrocarbons. Solutions of $\mathbf{5}$ fume when exposed to ambient conditions, but the solid 
can be briefly handled in air without significant hydrolysis. Crystals suitable for X-ray crystallography were grown by sublimation: 5 (250 mg) was placed in a PTFE-capped Oring sealed glass pressure vessel in a glove box and placed on top of an oven in a warm zone at approximately $50{ }^{\circ} \mathrm{C}$. After about 3 weeks, crystals of $\mathbf{5}$ deposited on the cooler end of the vessel. Crystals of the bis(etherate) were grown by slow cooling to $-45{ }^{\circ} \mathrm{C}$ of a solution of $\mathbf{5}$ in 1:1 diethyl ether/pentane; crystals of $\mathbf{6} \longleftarrow\left(\mathrm{OEt}_{2}\right)_{2}$ were deposited after 2 days.

\subsubsection{Thermogravimetric Analysis (TGA)}

TGA was performed on Pt pans with a TA Instruments Q50 housed in an MBraun Labmaster 130 dry-box filled with nitrogen gas ("4.8" 99.998\% purity). Pt pans were cleaned by sequential ultrasonication in dilute nitric acid (ca. $3 \mathrm{~N}$ ), water, then 2-propanol. They were then heated until red hot by a propane torch flame in air to remove remaining impurities. Ramp experiments were performed under a flow of ultrapure nitrogen ("5.0" $99.999 \%$ purity, $60 \mathrm{sccm}$ ) at $10{ }^{\circ} \mathrm{C} \mathrm{min}^{-1}$ to a maximum temperature of $500{ }^{\circ} \mathrm{C}$. Stress-tests were performed under the same conditions sequentially on freshly cleaned pans with incrementally increasing initial mass loadings.

Vapour pressures were estimated using a modified literature method. ${ }^{103}$ Vaporization constants $\alpha \mathrm{m}$ (from the Langmuir equation) were obtained for our instrument by calibrating compounds with known Antoine equation parameters and volatilities similar to the analyte. Benzoic acid $\left(\alpha_{l o T}=2.016 \times 10^{-4}\right)$ was used for high volatility compounds (i.e., 5), and $\mathrm{Cu}(\mathrm{tmhd})_{2}{ }^{388}\left(\alpha_{h i T}=4.512 \times 10^{-5}\right)$ was used for low volatility compounds (i.e., 3, 7, 8). The temperature program was set to jump by increments of $10{ }^{\circ} \mathrm{C}$, then hold that temperature for 7 minutes to allow the evaporating system to reach equilibrium, and then the derivative of mass with respect to temperature $\mathrm{dm} / \mathrm{dT}$ was taken from the linear regions of the isotherm steps for each temperature. These data were used to find the pressure $p$ as a function of $T$, thus $\ln \mathrm{p}$ as a function of $T^{-1}$, which can be modelled with the Clausius- 
Clapeyron equation. The slope of this line is equal to the enthalpy of sublimation (before melting) and evaporation (after melting) divided by the gas constant $\Delta H / R$.

\subsubsection{Atomic Layer Deposition Potential of 3}

The potential for 3 to form a thermally stable self-limiting monolayer under operando ALD conditions was evaluated in a commercial Picosun R-150 hot-wall viscous flow ALD reactor. Powdered samples of $\mathbf{3}$ (up to $10 \mathrm{~g}$ ) were loaded into an open-cup glass-lined stainless steel bubbler in a dry-box, capped with a rubber septum, then loaded into the bubbler heating jacket under a strong flow of ultrapure nirogen ("5.0" 99.999\%, $250 \mathrm{sccm}$ ). The bubbler and lines were evacuated to the base operating pressure of the reactor (ca. $10 \mathrm{hPa}$ ) to remove any water or oxygen that may have become trapped in the bubbler during loading. Then the bubbler and delivery lines were heated to $170{ }^{\circ} \mathrm{C}$ for $1 \mathrm{~h}$ before deposition to allow the precursor to melt and heat evenly. Precursor vapour was delivered into the deposition chamber with a "pulse": two pneumatic ALD valves were opened between the carrier gas inlet, the precursor, and the reactor, and nitrogen carrier gas was flowed over the heated precursor at $200 \mathrm{sccm}$ for $8 \mathrm{~s}$ under active vacuum, after which the valves were closed in reverse order. The lines and reactor chamber were purged with nitrogen carrier gas at $150 \mathrm{sccm}$ for $8 \mathrm{~s}$ to remove any unreacted 3 vapour and by-products of its self-assembly into a molecular layer on the surface.

During evaluation, several substrates were loaded into the deposition chamber: singleside polished "mechanical grade" high-purity hydrogen terminated single-crystalline (100) Si (RCA cleaned, sonicated in $2 \% \mathrm{HF}$ (aq) for $30 \mathrm{~min}$ immediately prior to use), $100 \mathrm{~nm}$ $\mathrm{Al}_{2} \mathrm{O}_{3}$ films (deposited by 900 cycles of ALD using trimethylaluminum and water at $250{ }^{\circ} \mathrm{C}$ on bare $\mathrm{Si}$ ), $100 \mathrm{~nm} \mathrm{SiO} 2$ (thermally grown on $\mathrm{Si}$ at $1200{ }^{\circ} \mathrm{C}$ in $\mathrm{O}_{2}$ ), and soda-lime glass microscope slides (cleaned by sonication for $30 \mathrm{~min}$ each in acetone, 2-propanol, and deionized water). No films could be observed after 2000 pulses of 3 at a reactor temperature of $400{ }^{\circ} \mathrm{C}$ by eye (evident as interference patterns on reflective, and colour changes on trans- 
parent substrates), ellipsometry or scanning electron microscopy (SEM). Preliminary ALD experiments using an oxidizing co-reagent (e.g., air) deposited uniform and transparent films. Above $420{ }^{\circ} \mathrm{C}$, an uncharacterized patchy thin film of mottled blue-grey material was observed on all substrates without co-reagent, indicating CVD growth behavior and the absence of ALD-like growth.

\subsubsection{X-ray Crystallography}

Crystals were attached to the tip of an appropriately sized $\mu \mathrm{m}$ MicroLoop with Paratone$\mathrm{N}$ oil. Measurements were made on a Bruker APEXII CCD equipped diffractometer (30 $\mathrm{mA}, 50 \mathrm{mV})$ using monochromated Mo $\mathrm{K} \alpha$ radiation $(\lambda=0.71073 \AA)$ at $125 \mathrm{~K}$. Bruker APEX2 software was used for the initial orientation and unit cells were indexed using a least-squares analysis of a random set of reflections collected from three series of $0.5^{\circ}$ wide scans, 10 seconds per frame and 12 frames per series that were well distributed in reciprocal space. For data collection, four $\omega$-scan frame series were collected with $0.5^{\circ}$ wide scans, 5 seconds frames and 366 frames per series at varying $\varphi$ angles $\left(\varphi=0^{\circ}, 90^{\circ}, 180^{\circ}\right.$, and $270^{\circ}$ ). The crystal-to-detector distance was set to $6 \mathrm{~cm}$ and a complete sphere of data was collected. Cell refinement and data reduction were performed with the Bruker APEX3 software, which corrects for beam inhomogeneity, possible crystal decay, Lorentz and polarization effects. Data processing and a multi-scan absorption correction was applied using the Bruker APEX3 software package. The structure was solved using intrinsic phasing $^{389}$ and all non-hydrogen atoms were refined anisotropically using SHELXL ${ }^{148}$ using a combination of the shelXle graphical user interface ${ }^{390}$ and OLEX2. ${ }^{391}$ Figures were made using UCSF Chimera ${ }^{150}$ and Adobe Photoshop CC 2018. CCDC 1885252-1885256 contains the supplementary crystallographic data for complexes $\mathbf{3}, \mathbf{5},\left(\mathbf{1} \leftarrow \mathrm{PMe}_{3}\right)_{6}, \mathbf{6} \leftarrow\left(\mathrm{OEt}_{2}\right)_{2}$, and 4, respectively. These data can be obtained free of charge from the Cambridge Crystallographic Database Centre via https://summary.ccdc.cam.ac.uk/structure-summary-form. Hexatin(II) di- $\mu_{3}$-oxy-octakis- $\mu$-trifluoroacetate (3): Two $-\mathrm{CF}_{3}$ groups were found to be 
best modeled using a two-component disorder model, one in a 66:34 ratio (on C8) and the other in a 73:27 (on C14). Details of crystal data, data collection, and structure refinement are listed in Table S1 in the freely-available Supplementary Information of the original paper.

Tin(IV) tetrakis(trifluoroacetate) (5): One of the $-\mathrm{CF}_{3}$ groups was modeled with a twocomponent disorder model in a 53:47 ratio. Details of crystal data, data collection, and structure refinement are listed in Table S4 in the freely-available Supplementary Information of the original paper.

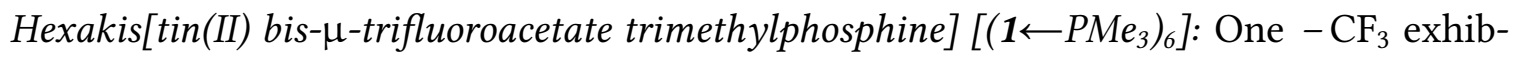
ited three-position disorder (53:33:14). Other $-\mathrm{CF}_{3}$ groups had larger thermal parameters, but attempts to model the disorder did not yield better models so they were left as is. One toluene molecule was found in the difference map and was successfully modelled. An-

other toluene model was found but attempts at modelling failed; the SQUEEZE routine was used and 48 electrons were removed, closely matching the expected 50 electrons for a toluene molecule. Details of crystal data, data collection, and structure refinement are listed in Table S6 in the freely-available Supplementary Information of the original paper. Tin(IV) tetrakis(trifluoroacetate)-trans-bis(diethyl ether) $\left[6 \longleftarrow\left(\mathrm{OEt}_{2}\right)_{2}\right]$ : Details of crystal data, data collection, and structure refinement are listed in Table S9 in the freely-available Supplementary Information of the original paper.

Tetratin(II) monotin(IV) di- $\mu_{3}$-oxy-octakis- $\mu$-trifluoroacetate (4): This compound was found to be a two-component inversion twin and was treated appropriately. Details of crystal data, data collection, and structure refinement are listed in Table S12 in the freely-available Supplementary Information of the original paper.

\subsubsection{Theoretical Calculations}

All theoretical calculations were carried out using the Gaussian09 Rev.D01 ${ }^{392}$ software suite. All geometry optimizations of $\mathrm{Sn}\left(\mathrm{O}_{2} \mathrm{CCF}_{3}\right)_{2}(\mathbf{1})$ and $\left.\mathrm{Sn}_{2} \mathrm{O}_{(} \mathrm{O}_{2} \mathrm{CCF}_{3}\right)_{2}$ (2) were per- 
formed in the $C_{2}$ symmetry point group, and $\mathrm{Sn}\left(\mathrm{O}_{2} \mathrm{CCF}_{3}\right)_{4}(\mathbf{6})$ was performed in the $D_{2 d}$ symmetry point group. The highest-level geometry optimizations accounted for scalar relativistic effects using the 2nd-order Douglas-Kroll-Hess (DKH) Hamiltonian ${ }^{393-396}$ and employed the SOGGA11-X ${ }^{397}$ density functional of Truhlar and Peverati, coupled with the all electron double- $\zeta$ and triple- $\zeta$-quality DZP-DKH and TZP-DKH basis sets of Jorge et al.. ${ }^{398-400}$ The point-nuclei model was used in the DKH treatment.

To establish flexible restraints for use in a SARACEN ${ }^{401-403}$ GED refinement, additional optimizations were carried out using the M06, ${ }^{404} \mathrm{M} 11,{ }^{405}$ and SOGGA11- $\mathrm{X}^{397} \mathrm{den}$ sity functionals coupled with the def2-SVP, def2-TZVP, and def2-QZVP basis sets. ${ }^{241,244}$ The characters of all optimized geometries were verified via vibrational frequency analysis and were confirmed to correspond to minima on the ground-state potential energy surface. Cartesian coordinates of all optimized geometries are found in Tables S18-S39 (for $\mathbf{1}$ and 2) and Tables S48-S58 (for 6) in the freely-available Supplementary Information of the original paper. Theoretical $r_{\mathrm{h} 1}$-type amplitudes of vibration $\left(u_{\mathrm{h} 1}\right)$ and curvilinear distance corrections $\left(k_{\mathrm{h} 1}\right)$ were generated for 1, 2, 6 from force fields computed at the SOGGA11-X/def2-SVP level using the SHRINK software package. ${ }^{406,407}$

\subsubsection{Gas-phase Electron Diffraction (GED)}

GED data for 1, 2, and 6 were acquired using the University of York gas electron diffractometer. ${ }^{408} \mathrm{An}$ accelerating potential of $42.22 \mathrm{kV}$ was applied to produce an electron emission current of $0.66 \mu \mathrm{A}$ and an electron wavelength of ca. $5.85 \mathrm{pm}$. The scattered electrons were recorded via exposures of image plates (Fuji BAS-IP MS 2025) at nozzle-to-imageplate distances of 234.5 and $477.0 \mathrm{~mm}$ for $\mathbf{1}$ and $\mathbf{2}$ and at nozzle-to-image-plate distances of 233.5 and $486.0 \mathrm{~mm}$ for $\mathbf{6}$. Five exposures were recorded at each nozzle-to-image-plate distance. To acquire data for $\mathbf{1}$ and $\mathbf{2}$, the sample and the nozzle were heated to 463 and $473 \mathrm{~K}$, respectively, during exposures at both nozzle-to-image-plate distances. To acquire data for $\mathbf{6}$, the sample and the nozzle were heated to 398 and $403 \mathrm{~K}$, respectively, during 
exposures at both nozzle-to-image-plate distances. A flatbed image plate scanner (Fuji BAS-1800II) was used to digitize the scattering intensities recorded on the image plates.

\subsubsection{Reduction and Refinement}

Digitized diffraction patterns were reduced to molecular intensity curves (MICs) using the data extraction package $x$ tract. ${ }^{409}$ MICs were refined using the ed@ed v3.0 least-squares refinement package, ${ }^{410}$ employing the electron scattering factors of Ross et al. ${ }^{411}$

The least-squares refinement procedures employed parameterized molecular models: one describing both 1 and 2, each within the $C_{2}$ symmetry point group, in terms of 23 refinable parameters comprising 11 distances $\left(p_{1}-p_{11}\right), 5$ angles $\left(p_{12}-p_{14}, p_{19}\right.$, and $\left.p_{23}\right)$ and 7 dihedral angles $\left(p_{15}-p_{18}\right.$ and $\left.p_{20}-p_{22}\right)$ with the option to weight the fraction of each 1 and 2, and one describing 6 within the $D_{2 d}$ symmetry point group in terms of 12 refinable parameters comprising 8 distances $\left(p_{1}-p_{8}\right)$ and 4 angles $\left(p_{9}-p_{12}\right)$. The FORTRAN90 code for the parametrized molecular models is available free-of-charge at DOI: 10.15124/5cb0c982-b280-47e9-9d46-08e675dfe0af.

The least-squares refinement procedures used the SARACEN approach and yielded internuclear distances of the $r_{\mathrm{h} 1}$ type; $r_{\mathrm{h} 1}$-type internuclear distances are related to the vibrationally-averaged $r_{a}$-type distances determined directly via the GED experiment by the relationship $r_{\mathrm{a} 3} \approx r_{a}+u_{\mathrm{h} 1}^{2} / r_{a}-k_{\mathrm{h} 1}$. SARACEN restraints were centered on values obtained from geometry optimizations at the SOGGA11-X/TZP-DKH level. For the refinement of $\mathbf{1}$ and $\mathbf{2}$, and for the refinement of $\mathbf{6}$, restraints were applied to 9 of the 23, and 7 of the 12, parameters, respectively, with the remaining parameters being allowed to refine free from restraint. 7 parameters remained unrefined after finalizing the refinement of $\mathbf{1}$ and 2; no parameters remained unrefined after finalizing the refinement of $\mathbf{6}$. Estimates of the uncertainties associated with the SARACEN restraint values were derived from sequential geometry optimizations using the M06, M11, and SOGGA11-X density functionals coupled with the def2-SVP, def2-TZVP, and def2-QZVP basis sets. 


\section{Chapter 7}

\section{Conclusions \& Future Directions}

Designing volatility, thermal stability, and chemical reactivity into molecules for use as precursors in atomic layer deposition (ALD) is not a problem with a single solution. Thermal stability and chemical stability, on one hand, are features that can be designed in with clear chemical rationality for main group elements and transition metals alike. Sterically bulky amido ligands with modifiable frameworks have allowed the isolation and demonstration of a diversity of molecules suitable as precursors to ALD, and in two cases have been developed into ALD processes with unique advantages. Ultralow-temperature ALD of PbS using diamido plumbylenes lead(II) bis[bis(trimethylsilyl)amide] (0Pb) and lead(II) rac- $N, N^{\prime}$-di-tert-butylbutane-2,3-diamide $(\mathbf{1 P b})$ with $\mathrm{H}_{2} \mathrm{~S}$ now allows for the protection of the popular and high-performance $\mathrm{CH}_{3} \mathrm{NH}_{3} \mathrm{PbI}_{3}$ (MAPI) perovskite from ambient conditions with a functional $p$-type material. Thermolysis of the precursors above $155^{\circ} \mathrm{C}$ and an unusual reaction between $\mathrm{H}_{2} \mathrm{~S}$ and $\mathbf{1 P b}$ limit their use to these low temperatures, however, which prompted another phase of precursor design. Following our previous success of blocking known decomposition pathways, we then found that geminal diaminosilanes act as excellent ligands for $\mathrm{Pb}(\mathrm{II})$ and can even stabilize $\mathrm{Co}(\mathrm{IV})$ enough to make a potential ALD precursor. From a different angle, we found that the inexpensive and commerciallyavailable trifluoroacetate forms thermally stable and volatile precursors with Sn(II) and $\mathrm{Sn}(\mathrm{IV})$, potentially enabling ALD of F-doped $\mathrm{SnO}_{x}$ (FTO) over a wide temperature range. 
In general, we were able to successfully design new and useful precursors for $\mathrm{Pb}(\mathrm{II})$, $\mathrm{Co}(\mathrm{IV}), \mathrm{Sn}(\mathrm{II})$ and $\mathrm{Sn}(\mathrm{IV})$ centers with a standard synthetic methodology.

On the other hand, this Dissertation has demonstrated that volatility does not yet have a clear chemical basis that can be used to rationally design it into precursors. For example, we showed some molecular features that one would seemingly need to avoid may in fact dramatically improve the volatility of molecules. The most striking examples were the polymeric $\operatorname{Sn}(\mathrm{II})$ and $\mathrm{Sn}(\mathrm{IV})$ trifluoroacetates, where the 1 Torr vapour pressure temperature was lowered by over $130{ }^{\circ} \mathrm{C}$ for the $\mathrm{Sn}(\mathrm{IV})$ derivative compared to its monomeric acetate analogue, thus opening the door to ultralow-temperature deposition of useful transparent conducting oxides. Quantum chemical analysis of a bulky heavy $\mathrm{Pb}$ (II) dimer with good volatility helped us highlight the molecular electrostatic potential (MEP) and its effect on volatility. In particular, the MEP also gave us a more nuanced picture of how decreasing polarity of a precursor increases its volatility, a previously trivial but unhelpfully general principle of precursor design. Thus, we found that the dimerization of 1,3-diaza-2-plumba-4-silacyclobutane monomers effectively neutralized their polar surface area and it should be expected that other less polar dimers with higher dissociation energies would be more volatile.

Unfortunately, due to the overlap between the global COVID-19 pandemic and the work performed in this thesis, many of the most interesting predictions could not be fully tested experimentally. We did not have the time to develop an ALD process with bis[lead(II) $N, N^{\prime}$-di-tert-butyl-1,1-dimethylsilanediamide] [(4Pb $\left.)_{2}\right]$ despite its excellent precursor properties, nor could we complete the synthetic work needed to verify our claims about the relative volatility of its derivatives. We also could not demonstrate ALD processes with the $\mathrm{Sn}(\mathrm{II})$ or $\mathrm{Sn}(\mathrm{IV})$ trifluoroacetates, nor with the Co(IV) bis(diamidosilane). Our preliminary results, however, suggest that $(\mathbf{4 P b})_{2}$ should be an effective precursor to $\mathrm{PbS}$ when used with $\mathrm{H}_{2} \mathrm{~S}$, that $\mathrm{Sn}(\mathrm{II})$ and $\mathrm{Sn}(\mathrm{IV})$ trifluoroacetates can deposit high-quality transparent conducting films for optoelectronics, and that unusual or even novel materi- 
als may be accessible with our $\mathrm{Co}(\mathrm{IV})$ precursor. These dearths provide the obvious first steps for future work on these precursors.

Beyond the obvious future work, there are some specific studies that must be completed to enable the use of MAPI, PbS, or FTO in third-generation photovoltaics (3GPVs). Firstly, the few-cycle ALD behaviour of the PbS processes using $\mathbf{0 P b}, \mathbf{1 P b}$ and $(\mathbf{4 P b})_{2}$ should be studied closely to quantify exactly how precisely we may tune the bandgap of $\mathrm{PbS}$ by quantum confinement and ALD. The deposition on different technologicallyrelevant nanowire arrays must also be explored, with particular attention given to the kinetic effects (i.e., change in film characteristics due to a different local environment within the confines of the NWA) on the ALD growth along the nanowires' length. Suitable heterojunction materials for dielectric barriers, and hole or electron conduction, deposited by ALD or other methods, must also be evaluated for use with ALD PbS. By extension, quantification of the heterojunctions' optoelectronic properties must be studied so that a device may be realized. Once the devices are possible, each process must be adapted to high-throughput high-volume manufacturing so that they may have industrial relevance. This will require precursors with sufficient volatility at atmospheric pressure to be used for spatial ALD, which has yet to be demonstrated for Pb-containing materials. We hope that the work presented in this Thesis may act as the starting point toward these future 3GPV devices. 


\section{References}

[1] Williams, R. Becquerel Photovoltaic Effect in Binary Compounds. The fournal of Chemical Physics 1960, 32, 1505-1514.

[2] Chapin, D. M.; Fuller, C. S.; Pearson, G. L. A New Silicon p-n Junction Photocell for Converting Solar Radiation into Electrical Power. Journal of Applied Physics 1954, $25,676-677$.

[3] Green, M. A. Third generation photovoltaics: Ultra-high conversion efficiency at low cost. Progress in Photovoltaics: Research and Applications 2001, 9, 123-135.

[4] Conibeer, G. Third-generation photovoltaics. Materials Today 2007, 10, 42-50.

[5] Green, M. A.; Dunlop, E. D.; Hohl-Ebinger, J.; Yoshita, M.; Kopidakis, N.; HoBaillie, A. W. Solar cell efficiency tables (Version 55). Progress in Photovoltaics: Research and Applications 2019, 28, 3-15.

[6] Britt, J.; Ferekides, C. Thin-film CdS/CdTe solar cell with $15.8 \%$ efficiency. Applied Physics Letters 1993, 62, 2851-2852.

[7] Repins, I.; Contreras, M. A.; Egaas, B.; DeHart, C.; Scharf, J.; Perkins, C. L.; To, B.; Noufi, R. 19.9\%-efficient $\mathrm{ZnO} / \mathrm{CdS} / \mathrm{CuInGaSe} 2$ solar cell with $81 \cdot 2 \%$ fill factor. Progress in Photovoltaics: Research and Applications 2008, 16, 235-239.

[8] Yablonovitch, E.; Miller, O. D.; Kurtz, S. R. The opto-electronic physics that broke the efficiency limit in solar cells. 2012 38th IEEE Photovoltaic Specialists Conference. 2012.

[9] Miller, O. D.; Yablonovitch, E.; Kurtz, S. R. Strong Internal and External Luminescence as Solar Cells Approach the Shockley-Queisser Limit. IEEE Journal of Photovoltaics 2012, 2, 303-311.

[10] O'Regan, B.; Grätzel, M. A low-cost, high-efficiency solar cell based on dyesensitized colloidal TiO2 films. Nature 1991, 353, 737-740.

[11] Inganäs, O. Organic Photovoltaics over Three Decades. Advanced Materials 2018, 30, 1800388.

[12] Ansari, M. I. H.; Qurashi, A.; Nazeeruddin, M. K. Frontiers, opportunities, and challenges in perovskite solar cells: A critical review. Fournal of Photochemistry and Photobiology C: Photochemistry Reviews 2018, 35, 1-24. 
[13] Nozik, A. J.; Beard, M. C.; Luther, J. M.; Law, M.; Ellingson, R. J.; Johnson, J. C. Semiconductor Quantum Dots and Quantum Dot Arrays and Applications of Multiple Exciton Generation to Third-Generation Photovoltaic Solar Cells. Chemical Reviews 2010, 110, 6873-6890.

[14] Hillhouse, H. W.; Beard, M. C. Solar cells from colloidal nanocrystals: Fundamentals, materials, devices, and economics. Current Opinion in Colloid \& Interface Science 2009, 14, 245-259.

[15] Ellingson, R. J.; Beard, M. C.; Johnson, J. C.; Yu, P.; Micic, O. I.; Nozik, A. J.; Shabaev, A.; Efros, A. L. Highly efficient multiple exciton generation in colloidal $\mathrm{PbSe}$ and $\mathrm{PbS}$ quantum dots. Nano Lett. 2005, 5, 865-871.

[16] Sukhovatkin, V.; Hinds, S.; Brzozowski, L.; Sargent, E. H. Colloidal quantum-dot photodetectors exploiting multiexciton generation. Science 2009, 324, 1542-1544.

[17] Nozik, A. J.; Miller, J. Introduction to Solar Photon Conversion. Chemical Reviews 2010, 110, 6443-6445.

[18] Alova, G. A global analysis of the progress and failure of electric utilities to adapt their portfolios of power-generation assets to the energy transition. Nature Energy 2020, 5, 920-927.

[19] Tsao, J.; Lewis, N.; Crabtree, G. Solar FAQs. Available at https://www.sandia.gov/ jytsao/Solar\%20FAQs.pdf (2006/4/20), 2006.

[20] Cobelo-García, A.; Filella, M.; Croot, P.; Frazzoli, C.; Laing, G. D.; OspinaAlvarez, N.; Rauch, S.; Salaun, P.; Schäfer, J.; Zimmermann, S. COST action TD1407: network on technology-critical elements (NOTICE)-from environmental processes to human health threats. Environmental Science and Pollution Research 2015, 22, 15188-15194.

[21] Hurd, A. J.; Kelley, R. L.; Eggert, R. G.; Lee, M.-H. Energy-critical elements for sustainable development. MRS Bulletin 2012, 37, 405-410.

[22] Kumar, A.; Zhou, C. The Race to Replace Tin-Doped Indium Oxide: Which Material Will Win? ACS Nano 2010, 4, 11.

[23] Beyer, W.; Hüpkes, J.; Stiebig, H. Transparent conducting oxide films for thin film silicon photovoltaics. Thin Solid Films 2007, 516, 147-154.

[24] Way, A.; Luke, J.; Evans, A. D.; Li, Z.; Kim, J.-S.; Durrant, J. R.; Lee, H. K. H.; Tsoi, W. C. Fluorine doped tin oxide as an alternative of indium tin oxide for bottom electrode of semi-transparent organic photovoltaic devices. AIP Advances 2019, 9, 085220 .

[25] Martínez, M.; Herrero, J.; Gutiérrez, M. Deposition of transparent and conductive Al-doped $\mathrm{ZnO}$ thin films for photovoltaic solar cells. Solar Energy Materials and Solar Cells 1997, 45, 75-86. 
[26] Jagadamma, L. K.; Al-Senani, M.; El-Labban, A.; Gereige, I.; Ndjawa, G. O. N.; Faria, J. C. D.; Kim, T.; Zhao, K.; Cruciani, F.; Anjum, D. H.; McLachlan, M. A.; Beaujuge, P. M.; Amassian, A. Polymer Solar Cells with Efficiency $>10 \%$ Enabled via a Facile Solution-Processed Al-Doped ZnO Electron Transporting Layer. Advanced Energy Materials 2015, 5, 1500204.

[27] Dong, J.; Zhao, Y.; Shi, J.; Wei, H.; Xiao, J.; Xu, X.; Luo, J.; Xu, J.; Li, D.; Luo, Y.; Meng, Q. Impressive enhancement in the cell performance of $\mathrm{ZnO}$ nanorod-based perovskite solar cells with Al-doped $\mathrm{ZnO}$ interfacial modification. Chem. Commun. 2014, 50, 13381-13384.

[28] Kinsey, N.; DeVault, C.; Kim, J.; Ferrera, M.; Shalaev, V. M.; Boltasseva, A. Epsilonnear-zero Al-doped $\mathrm{ZnO}$ for ultrafast switching at telecom wavelengths. Optica 2015, 2, 616.

[29] A decade of perovskite photovoltaics. Nature Energy 2019, 4, 1-1.

[30] Perovskites take steps to industrialization. Nature Energy 2020, 5, 1-1.

[31] Khenkin, M. V. et al. Consensus statement for stability assessment and reporting for perovskite photovoltaics based on ISOS procedures. Nature Energy 2020, 5, 35-49.

[32] Sutherland, B. R.; Hoogland, S.; Adachi, M. M.; Kanjanaboos, P.; Wong, C. T. O.; McDowell, J. J.; Xu, J.; Voznyy, O.; Ning, Z.; Houtepen, A. J.; Sargent, E. H. Perovskite Thin Films via Atomic Layer Deposition. Adv. Mater. 2015, 27, 53.

[33] Popov, G.; Mattinen, M.; Kemell, M. L.; Ritala, M.; Leskelä, M. Scalable Route to the Fabrication of $\mathrm{CH} 3 \mathrm{NH} 3 \mathrm{PbI} 3$ Perovskite Thin Films by Electrodeposition and Vapor Conversion. ACS Omega 2016, 1, 1296-1306.

[34] Popov, G.; Mattinen, M.; Hatanpää, T.; Vehkamäki, M.; Kemell, M.; Mizohata, K.; Räisänen, J.; Ritala, M.; Leskelä, M. Atomic Layer Deposition of PbI2 Thin Films. Chemistry of Materials 2019, 31, 1101-1109.

[35] Dasgupta, N. P.; Lee, W.; Prinz, F. B. Atomic layer deposition of lead sulfide thin films for quantum confinement. Chem. Mater. 2009, 21, 3973-3978.

[36] Zogg, H. Photovoltaic lead-chalcogenide on silicon infrared sensor arrays. Optical Engineering 1994, 33, 1440.

[37] Yuan, M.; Liu, M.; Sargent, E. H. Colloidal quantum dot solids for solution-processed solar cells. Nature Energy 2016, 1.

[38] Kovalenko, M. V. Opportunities and challenges for quantum dot photovoltaics. $\mathrm{Na}$ ture Nanotechnology 2015, 10, 994-997.

[39] Cao, W.; Zhang, Z.; Patterson, R.; Lin, Y.; Wen, X.; Veetil, B. P.; Zhang, P.; Zhang, Q.; Shrestha, S.; Conibeer, G.; Huang, S. Quantification of hot carrier thermalization in $\mathrm{PbS}$ colloidal quantum dots by power and temperature dependent photoluminescence spectroscopy. RSC Advances 2016, 6, 90846-90855. 
[40] Suntola, T. Atomic layer epitaxy. Thin Solid Films 1992, 216, 84-89.

[41] George, S. M. Atomic Layer Deposition: An Overview. Chemical Reviews 2010, 110, 111-131.

[42] Johnson, R. W.; Hultqvist, A.; Bent, S. F. A brief review of atomic layer deposition: from fundamentals to applications. Materials Today 2014, 17, 236-246.

[43] Poodt, P.; Lankhorst, A.; Roozeboom, F.; Spee, K.; Maas, D.; Vermeer, A. High-Speed Spatial Atomic-Layer Deposition of Aluminum Oxide Layers for Solar Cell Passivation. Advanced Materials 2010, 22, 3564-3567.

[44] Muñoz-Rojas, D.; MacManus-Driscoll, J. Spatial atmospheric atomic layer deposition: a new laboratory and industrial tool for low-cost photovoltaics. Mater. Horiz. 2014, 1, 314-320.

[45] Wu, Y.; Döhler, D.; Barr, M.; Oks, E.; Wolf, M.; Santinacci, L.; Bachmann, J. Atomic Layer Deposition from Dissolved Precursors. Nano Letters 2015, 15, 6379-6385.

[46] Koch, V. M.; Barr, M. K. S.; Büttner, P.; Mínguez-Bacho, I.; Döhler, D.; Winzer, B.; Reinhardt, E.; Segets, D.; Bachmann, J. A solution-based ALD route towards $(\mathrm{CH} 3 \mathrm{NH} 3)(\mathrm{PbI} 3)$ perovskite via lead sulfide films. Journal of Materials Chemistry A 2019, 7, 25112-25119.

[47] Monnier, B. P. L.; Wells, F.; Talebkeikhah, F.; Luterbacher, J. S. Atomic Layer Deposition on Dispersed Materials in Liquid Phase by Stoichiometrically Limited Injections. Advanced Materials 2019, 31, 1904276.

[48] Ithurria, S.; Talapin, D. V. Colloidal Atomic Layer Deposition (c-ALD) using SelfLimiting Reactions at Nanocrystal Surface Coupled to Phase Transfer between Polar and Nonpolar Media. Fournal of the American Chemical Society 2012, 134, 1858518590.

[49] Hazarika, A.; Fedin, I.; Hong, L.; Guo, J.; Srivastava, V.; Cho, W.; Coropceanu, I.; Portner, J.; Diroll, B. T.; Philbin, J. P.; Rabani, E.; Klie, R.; Talapin, D. V. Colloidal Atomic Layer Deposition with Stationary Reactant Phases Enables Precise Synthesis of "Digital" II-VI Nano-heterostructures with Exquisite Control of Confinement and Strain. Journal of the American Chemical Society 2019, 141, 13487-13496.

[50] Puurunen, R. L. (Invited) Learnings from an Open Science Effort: Virtual Project on the History of ALD. ECS Transactions 2018, 86, 3-17.

[51] Ahvenniemi, E. et al. Review Article: Recommended reading list of early publications on atomic layer deposition-Outcome of the "Virtual Project on the History of ALD”. Journal of Vacuum Science \& Technology A: Vacuum, Surfaces, and Films 2017, 35, 010801. 
[52] Malygin, A. A.; Drozd, V. E.; Malkov, A. A.; Smirnov, V. M. From V. B. Aleskovskii's "Framework" Hypothesis to the Method of Molecular Layering/Atomic Layer Deposition. Chemical Vapor Deposition 2015, 21, 216-240.

[53] Puurunen, R. L. A Short History of Atomic Layer Deposition: Tuomo Suntola's Atomic Layer Epitaxy. Chemical Vapor Deposition 2014, 20, 332-344.

[54] Cremers, V.; Puurunen, R. L.; Dendooven, J. Conformality in atomic layer deposition: Current status overview of analysis and modelling. Applied Physics Reviews 2019, 6, 021302 .

[55] Gordon, R.; Hausmann, D.; Kim, E.; Shepard, J. A Kinetic Model for Step Coverage by Atomic Layer Deposition in Narrow Holes or Trenches. Chemical Vapor Deposition 2003, 9, 73-78.

[56] Bae, C.; Kim, H.; Kim, E.; Park, H. G.; Shin, H. Atomic-Layer Deposition into 2versus 3-Dimensionally Ordered Nanoporous Media: Pore Size or Connectivity? Chemistry of Materials 2018, 30, 4748-4754.

[57] Lubitz, M.; Medina, P. A.; Antic, A.; Rosin, J. T.; Fahlman, B. D. Cost-Effective Systems for Atomic Layer Deposition. Fournal of Chemical Education 2014, 91, 10221027.

[58] de Paula, C.; Richey, N. E.; Zeng, L.; Bent, S. F. Mechanistic Study of Nucleation Enhancement in Atomic Layer Deposition by Pretreatment with Small Organometallic Molecules. Chemistry of Materials 2019, 32, 315-325.

[59] Baker, J. G.; Schneider, J. R.; Raiford, J. A.; de Paula, C.; Bent, S. F. Nucleation Effects in the Atomic Layer Deposition of Nickel-Aluminum Oxide Thin Films. Chemistry of Materials 2020, 32, 1925-1936.

[60] Ritala, M.; Niinistö, J. Industrial Applications of Atomic Layer Deposition. ECS Transactions 2019, 25, 641-652.

[61] Mistry, K. et al. A 45nm Logic Technology with High-k Metal Gate Transistors, Strained Silicon, $9 \mathrm{Cu}$ Interconnect Layers, 193nm Dry Patterning, and 100\% Pbfree Packaging. 2007 IEEE International Electron Devices Meeting. 2007.

[62] Bohr, M.; Chau, R.; Ghani, T.; Mistry, K. The High-k Solution. IEEE Spectrum 2007, $44,29-35$.

[63] Mackus, A. J. M.; Schneider, J. R.; MacIsaac, C.; Baker, J. G.; Bent, S. F. Synthesis of Doped, Ternary, and Quaternary Materials by Atomic Layer Deposition: A Review. Chemistry of Materials 2018, 31, 1142-1183.

[64] Imai, S.; Iizuka, T.; Sugiura, O.; Matsumura, M. Atomic layer epitaxy of Si using atomic H. Thin Solid Films 1993, 225, 168-172. 
[65] Sugahara, S.; Kitamura, T.; Imai, S.; Matsumura, M. Atomic layer epitaxy of germanium. Applied Surface Science 1994, 82-83, 380-386.

[66] Matsuyama, M.; Sugahara, S.; Ikeda, K.; Uchida, Y.; Matsumura, M. Hetero AtomicLayer Epitaxy of Ge on Si(100). Fapanese fournal of Applied Physics 2000, 39, 25362540 .

[67] Ritala, M. Atomic Layer Deposition of Oxide Thin Films with Metal Alkoxides as Oxygen Sources. Science 2000, 288, 319-321.

[68] Kessels, W. Database of ALD processes. 2019; https://www.atomiclimits.com/ alddatabase/.

[69] Puurunen, R. L. Surface chemistry of atomic layer deposition: A case study for the trimethylaluminum/water process. Journal of Applied Physics 2005, 97, 121301.

[70] Miikkulainen, V.; Leskelä, M.; Ritala, M.; Puurunen, R. L. Crystallinity of inorganic films grown by atomic layer deposition: Overview and general trends. F. Appl. Phys. 2013, 113, 2.

[71] Schmidt, J.; Merkle, A.; Brendel, R.; Hoex, B.; van de Sanden, M. C. M.; Kessels, W. M. M. Surface passivation of high-efficiency silicon solar cells by atomic-layerdeposited Al2O3. Progress in Photovoltaics: Research and Applications 2008, 16, 461466.

[72] O'Donnell, B. The weekend read: Atomic layer deposition storms market for PERC. Available at https://www.pv-magazine.com/2019/06/29/ the-weekend-read-atomic-layer-deposition-storms-market-for-perc/ (2019/06/29), 2019 .

[73] Bachmann, J. Atomic layer deposition in energy conversion applications; John Wiley \& Sons, 2017.

[74] van Delft, J. A.; Garcia-Alonso, D.; Kessels, W. M. M. Atomic layer deposition for photovoltaics: applications and prospects for solar cell manufacturing. Semiconductor Science and Technology 2012, 27, 074002.

[75] Niu, W.; Li, X.; Karuturi, S. K.; Fam, D. W.; Fan, H.; Shrestha, S.; Wong, L. H.; Tok, A. I. Y. Applications of atomic layer deposition in solar cells. Nanotechnology 2015, 26, 064001.

[76] Seo, S.; Jeong, S.; Park, H.; Shin, H.; Park, N.-G. Atomic layer deposition for efficient and stable perovskite solar cells. Chemical Communications 2019, 55, 2403-2416.

[77] Zardetto, V.; Williams, B. L.; Perrotta, A.; Giacomo, F. D.; Verheijen, M. A.; Andriessen, R.; Kessels, W. M. M.; Creatore, M. Atomic layer deposition for perovskite solar cells: research status, opportunities and challenges. Sustainable Energy \& Fuels 2017, 1, 30-55. 
[78] Bakke, J. R.; Pickrahn, K. L.; Brennan, T. P.; Bent, S. F. Nanoengineering and interfacial engineering of photovoltaics by atomic layer deposition. Nanoscale 2011, 3, 3482 .

[79] Meng, X.; Wang, X.; Geng, D.; Ozgit-Akgun, C.; Schneider, N.; Elam, J. W. Atomic layer deposition for nanomaterial synthesis and functionalization in energy technology. Materials Horizons 2017, 4, 133-154.

[80] Hossain, M. A.; Khoo, K. T.; Cui, X.; Poduval, G. K.; Zhang, T.; Li, X.; Li, W. M.; Hoex, B. Atomic layer deposition enabling higher efficiency solar cells: A review. Nano Materials Science 2020, 2, 204-226.

[81] Griffiths, M. B. E.; Dubrawski, Z. S.; Bačić, G.; Japahuge, A.; Masuda, J. D.; Zeng, T.; Barry, S. T. Controlling the Thermal Stability and Volatility of Organogold(I) Compounds for Vapor Deposition with Complementary Ligand Design. European fournal of Inorganic Chemistry 2019, 2019, 4927-4938.

[82] Griffiths, M. B. E.; Pallister, P. J.; Mandia, D. J.; Barry, S. T. Atomic Layer Deposition of Gold Metal. Chemistry of Materials 2015, 28, 44-46.

[83] Mäkelä, M.; Hatanpää, T.; Mizohata, K.; Räisänen, J.; Ritala, M.; Leskelä, M. Thermal Atomic Layer Deposition of Continuous and Highly Conducting Gold Thin Films. Chemistry of Materials 2017, 29, 6130-6136.

[84] Coyle, J. P.; Pallister, P. J.; Kurek, A.; Sirianni, E. R.; Yap, G. P. A.; Barry, S. T. Copper Iminopyrrolidinates: A Study of Thermal and Surface Chemistry. Inorganic Chemistry 2013, 52, 910-917.

[85] Coyle, J. P.; Sirianni, E. R.; Korobkov, I.; Yap, G. P. A.; Dey, G.; Barry, S. T. Study of Monomeric Copper Complexes Supported by N-Heterocyclic and Acyclic Diamino Carbenes. Organometallics 2017, 36, 2800-2810.

[86] Elliott, S. D. Improving ALD growth rate via ligand basicity: Quantum chemical calculations on lanthanum precursors. Surface and Coatings Technology 2007, 201, 9076-9081.

[87] Kazadojev, I.; Otway, D. J.; Elliott, S. D. Modeling of Precursors for Atomic Layer Deposition of Magnesium and Calcium Oxide. Chemical Vapor Deposition 2013, 19, 117-124.

[88] Dey, G.; Wrench, J. S.; Hagen, D. J.; Keeney, L.; Elliott, S. D. Quantum chemical and solution phase evaluation of metallocenes as reducing agents for the prospective atomic layer deposition of copper. Dalton Transactions 2015, 44, 10188-10199.

[89] Fang, G.; Xu, L.; Cao, Y.; Li, A. Theoretical design and computational screening of precursors for atomic layer deposition. Coordination Chemistry Reviews 2016, 322, 94-103. 
[90] Elliott, S. D.; Dey, G.; Maimaiti, Y. Classification of processes for the atomic layer deposition of metals based on mechanistic information from density functional theory calculations. The fournal of Chemical Physics 2017, 146, 052822.

[91] Filatova, E. A.; Hausmann, D.; Elliott, S. D. Investigating routes toward atomic layer deposition of silicon carbide: Ab initio screening of potential silicon and carbon precursors. Journal of Vacuum Science \& Technology A: Vacuum, Surfaces, and Films 2017, 35, 01B103.

[92] Crutchley, R. J. CVD and ALD precursor design and application. Coordination Chemistry Reviews 2013, 257, 3153.

[93] Johnson, A. L.; Parish, J. D. Organometallic Chemistry; Royal Society of Chemistry, 2018; pp 1-53.

[94] Persad, A. H.; Ward, C. A. Expressions for the Evaporation and Condensation Coefficients in the Hertz-Knudsen Relation. Chemical Reviews 2016, 116, 7727-7767.

[95] Zudin, Y. B. Non-equilibrium Evaporation and Condensation Processes; Springer International Publishing, 2019.

[96] Vogt, P. S.; Liapine, R.; Kirchner, B.; Dyson, A. J.; Huber, H.; Marcelli, G.; Sadus, R. J. Molecular simulation of the vapour-liquid phase coexistence of neon and argon using ab initio potentials. Physical Chemistry Chemical Physics 2001, 3, 1297-1302.

[97] Musolino, N.; Trout, B. L. Insight into the molecular mechanism of water evaporation via the finite temperature string method. The fournal of Chemical Physics 2013, $138,134707$.

[98] Nagata, Y.; Usui, K.; Bonn, M. Molecular Mechanism of Water Evaporation. Physical Review Letters 2015, 115.

[99] Zhakhovsky, V. V.; Kryukov, A. P.; Levashov, V. Y.; Shishkova, I. N.; Anisimov, S. I. Mass and heat transfer between evaporation and condensation surfaces: Atomistic simulation and solution of Boltzmann kinetic equation. Proceedings of the National Academy of Sciences 2018, 116, 18209-18217.

[100] Xiao, B.; Stixrude, L. Critical vaporization of MgSiO3. Proceedings of the National Academy of Sciences 2018, 115, 5371-5376.

[101] Yakubovich, A. V.; Son, W.-J.; Kwon, O.; Choi, H.; Choi, B.; Kim, S. Accurate Vapor Pressure Prediction for Large Organic Molecules: Application to Materials Utilized in Organic Light-Emitting Diodes. Fournal of Chemical Theory and Computation 2020, 16, 5845-5851.

[102] Price, D. M. Vapor pressure determination by thermogravimetry. Thermochimica Acta 2001, 367-368, 253 - 262, Material Chaaracterization by Thermal Analytical Methods. 
[103] Kunte, G. V.; Shivashankar, S. A.; Umarji, A. M. Thermogravimetric evaluation of the suitability of precursors for MOCVD. Measurement Science and Technology 2008, 19, 025704.

[104] Vyazovkin, S.; Burnham, A. K.; Favergeon, L.; Koga, N.; Moukhina, E.; PérezMaqueda, L. A.; Sbirrazzuoli, N. ICTAC Kinetics Committee recommendations for analysis of multi-step kinetics. Thermochimica Acta 2020, 689, 178597.

[105] Dollimore, D. A breath of fresh air. Thermochimica Acta 1999, 340-341, 19 - 29.

[106] Coats, A. W.; Redfern, J. Kinetic parameters from thermogravimetric data. Nature 1964, 201, 68-69.

[107] Wilburn, F. The determination of kinetic parameters from DTG curves - fact or fiction? Thermochimica Acta 1999, 340-341, 77 - 87.

[108] Kissinger, H. E. Reaction Kinetics in Differential Thermal Analysis. Analytical Chemistry 1957, 29, 1702-1706.

[109] Hoffmann-Eifert, S.; Watanabe, T. In Atomic Layer Deposition for Semiconductors; Hwang, C. S., Ed.; Springer US: Boston, MA, 2014; pp 149-171.

[110] Ono, L. K.; Juarez-Perez, E. J.; Qi, Y. Progress on Perovskite Materials and Solar Cells with Mixed Cations and Halide Anions. ACS Appl. Mater. Interfaces 2017, 9, 30197-30246.

[111] Miller, E. M.; Kroupa, D. M.; Zhang, J.; Schulz, P.; Marshall, A. R.; Kahn, A.; Lany, S.; Luther, J. M.; Beard, M. C.; Perkins, C. L.; van de Lagemaat, J. Revisiting the Valence and Conduction Band Size Dependence of PbS Quantum Dot Thin Films. ACS Nano 2016, 10, 3302-3311.

[112] Saran, R.; Curry, R. J. Lead sulphide nanocrystal photodetector technologies. Nature Photonics 2016, 10, 81.

[113] Krisyuk, V. V.; Turgambaeva, A. E.; Igumenov, I. K. Volatile Lead $\beta$-Diketonates as CVD Precursors. Chem. Vap. Deposition 1998, 4, 43-46.

[114] Ramesh, R.; Aggarwal, S.; Auciello, O. Science and technology of ferroelectric films and heterostructures for non-volatile ferroelectric memories. Mater. Sci. Eng., $R$ 2001, 32, 191-236.

[115] Asay, M.; Jones, C.; Driess, M. N-Heterocyclic carbene analogues with low-valent group 13 and group 14 elements: syntheses, structures, and reactivities of a new generation of multitalented ligands. Chem. Rev 2011, 111, 354-396.

[116] Blom, B.; Gallego, D.; Driess, M. N-heterocyclic silylene complexes in catalysis: new frontiers in an emerging field. Inorg. Chem. Front. 2014, 1, 134-148. 
[117] Benedek, Z.; Szilvási, T. Theoretical assessment of low-valent germanium compounds as transition metal ligands: Can they be better than phosphines or NHCs? Organometallics 2017, 36, 1591-1600.

[118] Vepřek, S.; Prokop, J.; Glatz, F.; Merica, R.; Klingan, F.; Herrmann, W. Organometallic chemical vapor deposition of germanium from a cyclic germylene, 1, 3-Di-tertbutyl-1, 3, 2-diazagermolidin-2-ylidine. Chem. Mater. 1996, 8, 825-831.

[119] Kim, S. B.; Sinsermsuksakul, P.; Pike, R. D.; Gordon, R. G. Synthesis of NHeterocyclic Stannylene (Sn (II)) and Germylene (Ge (II)) and a Sn (II) Amidinate and Their Application as Precursors for Atomic Layer Deposition. Chem. Mater. 2014, 26, 3065-3073.

[120] Gordon, R. G.; Hock, A. S.; Heo, J.; Sinsermsuksakul, P. Cyclic Metal Amides and Vapor Deposition Using Them. patentus 8,796,483, 2014.

[121] Gans-Eichler, T.; Gudat, D.; Nieger, M. Tin Analogues of "Arduengo Carbenes": Synthesis of 1,3,2 $\lambda^{2}$-diazastannoles and transfer of Sn atoms between a 1,3,2 $\lambda^{2}$ diazastannole and a diazadiene. Angew. Chem., Int. Ed. 2002, 41, 1888-1891.

[122] Hahn, F. E.; Heitmann, D.; Pape, T. Synthesis and characterization of stable NHeterocyclic plumbylenes. Eur. F. Inorg. Chem. 2008, 2008, 1039-1041.

[123] Charmant, J. P.; Haddow, M. F.; Hahn, F. E.; Heitmann, D.; Fröhlich, R.; Mansell, S. M.; Russell, C. A.; Wass, D. F. Syntheses and molecular structures of some saturated N-heterocyclic plumbylenes. Dalton Trans. 2008, 6055-6059.

[124] Artus, G. CCDC 123898. CSD Communication 1996,

[125] Coyle, J. P.; Gordon, P. G.; Wells, A. P.; Mandia, D. J.; Sirianni, E. R.; Yap, G. P.; Barry, S. T. Thermally robust gold and silver iminopyrrolidinates for chemical vapor deposition of metal films. Chem. Mater. 2013, 25, 4566-4573.

[126] Horchler, K.; Stader, C.; Wrackmeyer, B. Monomeric 1,4,2,3,5 $\lambda^{2}$ diazadisilastannolidines and plumbolidines. Inorg. Chim. Acta 1986, 117, L39-L40.

[127] Wrackmeyer, B.; Stader, C.; Horchler, K.; Zhou, H.; Schlosser, D. Dimeric cyclic bis(amino) stannylenes and plumbylenes-a multinuclear magnetic resonance study. Inorg. Chim. Acta 1990, 176, 205-214.

[128] Stader, C.; Wrackmeyer, B. Anwendung der 15N-NMR-Spektroskopie zur Charakterisierung reaktiver, koordinativ ungesättigter Zinn-, Bleiund PhosphorStickstoff-Verbindungen/Application of 15N-NMR Spectroscopy to the Characterization of Reactive, Coordinatively Unsaturated Tin-, Lead-and PhosphorusNitrogen Compounds. Zeitschrift für Naturforschung B 1987, 42, 1515-1519.

[129] Li, W.; Hill, N. J.; Tomasik, A. C.; Bikzhanova, G.; West, R. A new monomeric saturated N-heterocyclic silylene as a racemic mixture. Organometallics 2006, 25, 38023805 . 
[130] Haaf, M.; Schmedake, T. A.; West, R. Stable silylenes. Acc. Chem. Res. 2000, 33, 704714.

[131] Arduengo III, A. J.; Bock, H.; Chen, H.; Denk, M.; Dixon, D. A.; Green, J. C.; Herrmann, W. A.; Jones, N. L.; Wagner, M.; West, R. Photoelectron spectroscopy of a carbene/silylene/germylene series. F. Am. Chem. Soc. 1994, 116, 6641-6649.

[132] Blom, B.; Said, A.; Szilvási, T.; Menezes, P. W.; Tan, G.; Baumgartner, J.; Driess, M. Alkaline-earth-metal-induced liberation of rare allotropes of elemental silicon and germanium from $N$-heterocyclic metallylenes. Inorg. Chem. 2015, 54, 8840-8848.

[133] Gans-Eichler, T.; Gudat, D.; Nättinen, K.; Nieger, M. The Transfer of Tin and Germanium Atoms from N-Heterocyclic Stannylenes and Germylenes to Diazadienes. Chem. Eur. F. 2006, 12, 1162-1173.

[134] Herrmann, W. A.; Denk, M.; Behm, J.; Scherer, W.; Klingan, F.-R.; Bock, H.; Solouki, B.; Wagner, M. Stable cyclic germanediyls ("cyclogermylenes"): Synthesis, structure, metal complexes, and thermolyses. Angew. Chem., Int. Ed. 1992, 31, 1485-1488.

[135] Guzei, I. A.; Timokhin, V. I.; West, R. [Bis (trimethylsilyl) amido- $\kappa$ N] \{tert-butyl [(E)2 -(tert-butylimino) ethyl] amido- $\left.\kappa 2 \mathrm{~N}, \mathrm{~N}^{\prime}\right\}$ tin (II), a key intermediate in the synthesis of 1, 3-di-tert-butyl-2, 3-dihydro-1H-1, 3, 2-diazastannole. Acta Crystallogr. C 2006, 62, m90-m92.

[136] Martelli, G.; Morri, S.; Savoia, D. Synthesis of Chiral C2-Symmetric 1, 2-Diamines by the Addition of Organolithium Reagents to N, N'-Bis [(S)-1-phenylethyl] ethanediimine. Tetrahedron 2000, 56, 8367-8374.

[137] Schmidt, A.-K. C.; Stark, C. B. The Glycol Cleavage in Natural Product Synthesis: Reagent Classics and Recent Advances. Synthesis 2014, 46, 3283-3308.

[138] Criegee, R.; Kraft, L.; Rank, B. Die Glykolspaltung, ihr Mechanismus und ihre Anwendung auf chemische Probleme. Justus Liebigs Ann Chem 1933, 507, 159-197.

[139] Fjeldberg, T.; Hope, H.; Lappert, M. F.; Power, P. P.; Thorne, A. J. Molecular structures of the main group 4 metal (II) bis(trimethylsilyl)-amides $\mathrm{M}\left[\mathrm{N}\left(\mathrm{SiMe}_{3}\right)_{2}\right]_{2}$ in the crystal (X-ray) and vapour (gas-phase electron diffraction). F. Chem. Soc., Chem. Commun. 1983, 639-641.

[140] Heitmann, D.; Pape, T.; Hepp, A.; Mück-Lichtenfeld, C.; Grimme, S.; Hahn, F. E. Palladium and platinum complexes of a benzannulated n-heterocyclic plumbylene with an unusual bonding mode. F. Am. Chem. Soc. 2011, 133, 11118-11120.

[141] Wrackmeyer, B. Application of 207Pb NMR parameters; Annual Reports on NMR Spectroscopy; Academic Press, 2002; Vol. 47; pp 1 - 37. 
[142] Coyle, J. P.; Kurek, A.; Pallister, P. J.; Sirianni, E. R.; Yap, G. P.; Barry, S. T. Preventing thermolysis: precursor design for volatile copper compounds. Chem. Commun. 2012, 48, 10440-10442.

[143] Mann, S.; Carillon, S.; Breyne, O.; Marquet, A. Total synthesis of amiclenomycin, an inhibitor of biotin biosynthesis. Chem. Eur. F. 2002, 8, 439-450.

[144] Gardiner, M. G.; Raston, C. L. Carbolithiations of N, N'-Di-tert-butyl-1,4-diazabuta1,3-diene. Inorg. Chem. 1995, 34, 4206-4212.

[145] Amonoo-Neizer, E.; Shaw, R.; Skovlin, D.; Smith, B.; Rosenthal, J. W.; Jolly, W. L. Lithium bis(trimethylsilyl) amide and tris(trimethylsilyl) amine. Inorg. Synth. 1966, $8,19-22$.

[146] Harris, D. H.; Lappert, M. F. Monomeric, volatile bivalent amides of group IV B elements, $\mathrm{M}\left(\mathrm{NR}_{2}{ }^{1}\right)_{2}$ and $\mathrm{M}\left(\mathrm{NR}^{1} \mathrm{R}^{2}\right)_{2}\left(\mathrm{M}=\mathrm{Ge}\right.$, Sn, or $\left.\mathrm{Pb} ; \mathrm{R}^{1}=\mathrm{Me}_{3} \mathrm{Si}, \mathrm{R}^{2}=\mathrm{Me}_{3} \mathrm{C}\right) . \mathcal{F}$. Chem. Soc., Chem. Commun. 1974, 895-896.

[147] Lappert, M. F.; Slade, M. J.; Singh, A.; Atwood, J. L.; Rogers, R. D.; Shakir, R. Structure and reactivity of sterically hindered lithium amides and their diethyl etherates: crystal and molecular structures of $\left[\mathrm{Li}\left\{\mathrm{N}\left(\mathrm{SiMe}_{3}\right)_{2}\right\}\left(\mathrm{OEt}_{2}\right)\right]_{2}$ and tetrakis $(2,2,6$, 6tetramethylpiperidinatolithium). f. Am. Chem. Soc. 1983, 105, 302-304.

[148] Sheldrick, G. M. Crystal structure refinement with SHELXL. Acta Crystallographica Section C: Structural Chemistry 2015, 71, 3-8.

[149] Spek, A. L. Structure validation in chemical crystallography. Acta Crystallographica Section D: Biological Crystallography 2009, 65, 148-155.

[150] Pettersen, E. F.; Goddard, T. D.; Huang, C. C.; Couch, G. S.; Greenblatt, D. M.; Meng, E. C.; Ferrin, T. E. UCSF Chimera-a Visualization System for Exploratory Research and Analysis. F. Comput. Chem. 2004, 25, 1605.

[151] Bloem, J.; Kröger, F. A. A Relation between Hardness and Stoichiometry in Lead Sulphide Single Crystals. Nature 1955, 175, 861.

[152] Wise, F. W. Lead Salt Quantum Dots: The Limit of Strong Quantum Confinement. Acc. Chem. Res. 2000, 33, 773.

[153] Moon, D. G.; Rehan, S.; Yeon, D. H.; Lee, S. M.; Park, S. J.; Ahn, S. J.; Cho, Y. S. A Review on Binary Metal Sulfide Heterojunction Solar Cells. Sol. Energy Mater. Sol. Cells 2019, 200, 109963.

[154] Yeon, D. H.; Mohanty, B. C.; Lee, C. Y.; Lee, S. M.; Cho, Y. S. High-Efficiency Double Absorber PbS/CdS Heterojunction Solar Cells by Enhanced Charge Collection Using a ZnO Nanorod Array. ACS Omega 2017, 2, 4894. 
[155] Safrani, T.; Kumar, T. A.; Klebanov, M.; Arad-Vosk, N.; Beach, R.; Sa'Ar, A.; Abdulhalim, I.; Sarusi, G.; Golan, Y. Chemically Deposited PbS Thin Film PhotoConducting Layers for Optically Addressed Spatial Light Modulators. F. Mater. Chem. C 2014, 2, 9132.

[156] Lee, S. M.; Jang, W.; Mohanty, B. C.; Yoo, J.; Jang, J. W.; Kim, D. B.; Yi, Y.; Soon, A.; Cho, Y. S. Experimental Demonstration of in Situ Stress-Driven Optical Modulations in Flexible Semiconducting Thin Films with Enhanced Photodetecting Capability. Chem. Mater. 2018, 30, 7776.

[157] Carrillo-Castillo, A.; Salas-Villasenor, A.; Mejia, I.; Aguirre-Tostado, S.; Gnade, B. E.; Quevedo-López, M. A. P-Type Thin Films Transistors with Solution-Deposited Lead Sulfide Films as Semiconductor. Thin Solid Films 2012, 520, 3107.

[158] Navale, S. T.; Bandgar, D. K.; Chougule, M. A.; Patil, V. B. Facile Method of Preparation of PbS Films for NO2 Detection. RSC Adv. 2015, 5, 6518.

[159] Beatriceveena, T. V.; Prabhu, E.; Sree Rama Murthy, A.; Jayaraman, V.; Gnanasekar, K. I. Highly Selective PbS Thin Film Based Ammonia Sensor for Inert Ambient: In-Situ Hall and Photoelectron Studies. Appl. Surf. Sci. 2018, 456, 430.

[160] Nykänen, E.; Laine-Ylijoki, J.; Soininen, P.; Niinistö, L.; Leskelä, M.; HubertPfalzgraf, L. G. Growth of PbS Thin Films from Novel Precursors by Atomic Layer Epitaxy. F. Mater. Chem. 1994, 4, 1409.

[161] Dasgupta, N. P.; Jung, H. J.; Trejo, O.; McDowell, M. T.; Hryciw, A.; Brongersma, M.; Sinclair, R.; Prinz, F. B. Atomic Layer Deposition of Lead Sulfide Quantum Dots on Nanowire Surfaces. Nano Letters 2011, 11, 934-940.

[162] Jena, A. K.; Kulkarni, A.; Miyasaka, T. Halide Perovskite Photovoltaics: Background, Status, and Future Prospects. Chem. Rev. 2019, 119, 3036.

[163] Chen, Q. et al. All-Inorganic Perovskite Nanocrystal Scintillators. Nature 2018, 561, 88.

[164] Dong, Y.; Zou, Y.; Song, J.; Song, X.; Zeng, H. Recent Progress of Metal Halide Perovskite Photodetectors. F. Mater. Chem. C 2017, 5, 11369.

[165] Chen, J.; Zhou, S.; Jin, S.; Li, H.; Zhai, T. Crystal Organometal Halide Perovskites with Promising Optoelectronic Applications. F. Mater. Chem. C 2016, 4, 11.

[166] Sim, K.; Jun, T.; Bang, J.; Kamioka, H.; Kim, J.; Hiramatsu, H.; Hosono, H. Performance Boosting Strategy for Perovskite Light-Emitting Diodes. Appl. Phys. Rev. 2019, 6, 031402.

[167] Yu, W. et al. Single Crystal Hybrid Perovskite Field-Effect Transistors. Nat. Commun. 2018, 9, 9. 
[168] Kang, K.; Ahn, H.; Song, Y.; Lee, W.; Kim, J.; Kim, Y.; Yoo, D.; Lee, T. Perovskite Memory Devices: High-Performance Solution-Processed Organo-Metal Halide Perovskite Unipolar Resistive Memory Devices in a Cross-Bar Array Structure. Adv. Mater. 2019, 31, 1970149.

[169] Xu, Z.; Liu, Z.; Huang, Y.; Zheng, G.; Chen, Q.; Zhou, H. To Probe the Performance of Perovskite Memory Devices: Defects Property and Hysteresis. F. Mater. Chem. C 2017, 5, 5810 .

[170] Koushik, D.; Verhees, W. J. H.; Kuang, Y.; Veenstra, S.; Zhang, D.; Verheijen, M. A.; Creatore, M.; Schropp, R. E. I. High-Efficiency Humidity-Stable Planar Perovskite Solar Cells Based on Atomic Layer Architecture. Energy Environ. Sci. 2017, 10, 91.

[171] Zardetto, V.; Williams, B. L.; Perrotta, A.; Di Giacomo, F.; Verheijen, M. A.; Andriessen, R.; Kessels, W. M. M.; Creatore, M. Atomic Layer Deposition for Perovskite Solar Cells: Research Status, Opportunities and Challenges. Sustain. Energy Fuels 2017, 1,30 .

[172] Brinkmann, K. O.; Gahlmann, T.; Riedl, T. Atomic Layer Deposition of Functional Layers in Planar Perovskite Solar Cells. Sol. RRL 2020, 4, 1900332.

[173] Raiford, J. A.; Oyakhire, S. T.; Bent, S. F. Applications of Atomic Layer Deposition and Chemical Vapor Deposition for Perovskite Solar Cells. Energy Environ. Sci. 2020, 13, 1997.

[174] Yu, X.; Yan, H.; Peng, Q. Improve the Stability of Hybrid Halide Perovskite via Atomic Layer Deposition on Activated Phenyl-C61 Butyric Acid Methyl Ester. ACS Appl. Mater. Interfaces 2018, 10, 28948.

[175] Lee, Y.; Lee, S.; Seo, G.; Paek, S.; Cho, K. T.; Huckaba, A. J.; Calizzi, M.; Choi, D.; Park, J.-S.; Lee, D.; Lee, H. J.; Asiri, A. M.; Nazeeruddin, M. K. Efficient Planar Perovskite Solar Cells Using Passivated Tin Oxide as an Electron Transport Layer. Adv. Sci. 2018, 5, 1800130.

[176] Yu, X.; Yan, H.; Peng, Q. Improve the Stability of Hybrid Halide Perovskite via Atomic Layer Deposition on Activated Phenyl-C61 Butyric Acid Methyl Ester. ACS Applied Materials \& Interfaces 2018, 10, 28948-28954.

[177] Lee, Y.; Lee, S.; Seo, G.; Paek, S.; Cho, K. T.; Huckaba, A. J.; Calizzi, M.; won Choi, D.; Park, J.-S.; Lee, D.; Lee, H. J.; Asiri, A. M.; Nazeeruddin, M. K. Efficient Planar Perovskite Solar Cells Using Passivated Tin Oxide as an Electron Transport Layer. Advanced Science 2018, 5, 1800130.

[178] Zheng, X.; Lei, H.; Yang, G.; Ke, W.; Chen, Z.; Chen, C.; Ma, J.; Guo, Q.; Yao, F.; Zhang, Q.; Xu, H.; Fang, G. Enhancing Efficiency and Stability of Perovskite Solar Cells via a High Mobility P-Type PbS Buffer Layer. Nano Energy 2017, 38, 1. 
[179] Xie, L.; Zhang, T.; Zhao, Y. Stabilizing the MAPbI3 Perovksite via the In-Situ Formed Lead Sulfide Layer for Efficient and Robust Solar Cells. F. Energy Chem. 2020, 47, 62.

[180] Sutherland, B. R.; Hoogland, S.; Adachi, M. M.; Wong, C. T. O.; Sargent, E. H. Conformal Organohalide Perovskites Enable Lasing on Spherical Resonators. ACS Nano 2014, 8, 10947.

[181] Yun, S. J.; Kim, Y. S.; Park, S.-H. K. Fabrication of CaS:Pb blue phosphor by incorporating dimeric $\mathrm{Pb}(2+)$ luminescent centers. Applied Physics Letters 2001, 78, 721-723.

[182] Bačić, G.; Zanders, D.; Mallick, B.; Devi, A.; Barry, S. T. Designing Stability into Thermally Reactive Plumbylenes. Inorganic Chemistry 2018, 57, 8218-8226, PMID: 29943579.

[183] Crowe, L. L.; Tolbert, L. M. Silica Passivation Efficiency Monitored by a SurfaceBound Fluorescent Dye. Langmuir 2008, 24, 8541.

[184] Mattinen, M.; King, P. J.; Khriachtchev, L.; Meinander, K.; Gibbon, J. T.; Dhanak, V. R.; Räisänen, J.; Ritala, M.; Leskelä, M. Low-Temperature Wafer-Scale Deposition of Continuous 2D SnS2 Films. Small 2018, 14, 1800547.

[185] Mattinen, M.; Popov, G.; Vehkamäki, M.; King, P. J.; Mizohata, K.; Jalkanen, P.; Räisänen, J.; Leskelä, M.; Ritala, M. Atomic Layer Deposition of Emerging 2D Semiconductors, HfS2 and ZrS2, for Optoelectronics. Chem. Mater. 2019, 31, 5713.

[186] Huang, Y.; Liu, L.; Zhao, W.; Chen, Y. Preparation and Characterization of Molybdenum Disulfide Films Obtained by One-Step Atomic Layer Deposition Method. Thin Solid Films 2017, 624, 101.

[187] Hämäläinen, J.; Mattinen, M.; Mizohata, K.; Meinander, K.; Vehkamäki, M.; Räisänen, J.; Ritala, M.; Leskelä, M. Atomic Layer Deposition of Rhenium Disulfide. $A d v$. Mater. 2018, 30, 1703622.

[188] Tripathi, T. S.; Lahtinen, J.; Karppinen, M. Atomic Layer Deposition of Conducting CuS Thin Films from Elemental Sulfur. Adv. Mater. Interfaces 2018, 5, 1701366.

[189] Linstrom, P. NIST Chemistry WebBook, NIST Standard Reference Database 69. 1997; http://webbook.nist.gov/chemistry/.

[190] Wei, Q.; Mukaida, M.; Naitoh, Y.; Ishida, T. Morphological Change and Mobility Enhancement in PEDOT:PSS by Adding Co-Solvents. Adv. Mater. 2013, 25, 2831.

[191] Snaith, H. J.; Grätzel, M. Enhanced Charge Mobility in a Molecular Hole Transporter via Addition of Redox Inactive Ionic Dopant: Implication to Dye-Sensitized Solar Cells. Appl. Phys. Lett. 2006, 89, 262114. 
[192] Chia-Ching, W.; Cheng-Fu, Y. Investigation of the Properties of Nanostructured LiDoped NiO Films Using the Modified Spray Pyrolysis Method. Nanoscale Res. Lett. 2013, 8, 33.

[193] Orton, J. W.; Powell, M. J. The Hall Effect in Polycrystalline and Powdered Semiconductors. Rep. Prog. Phys. 1980, 43, 1263.

[194] Reid, O. G.; Yang, M.; Kopidakis, N.; Zhu, K.; Rumbles, G. Grain-Size-Limited Mobility in Methylammonium Lead Iodide Perovskite Thin Films. ACS Energy Lett. 2016, 1,561 .

[195] Kothiyal, G.; Ghosh, B. On Conductivity in Lead Chalcogenides. Prog. Cryst. Growth Charact. Mater. 1990, 20, 313.

[196] Allgaier, R. S.; Scanlon, W. W. Mobility of Electrons and Holes in PbS, PbSe, and PbTe between Room Temperature and 4.2 K. Phys. Rev. 1958, 111, 1029.

[197] Oh, S. J.; Berry, N. E.; Choi, J.-H.; Gaulding, E. A.; Paik, T.; Hong, S.-H.; Murray, C. B.; Kagan, C. R. Stoichiometric Control of Lead Chalcogenide Nanocrystal Solids to Enhance Their Electronic and Optoelectronic Device Performance. ACS Nano 2013, 7, 2413.

[198] Ertekin, E.; Srinivasan, V.; Ravichandran, J.; Rossen, P. B.; Siemons, W.; Majumdar, A.; Ramesh, R.; Grossman, J. C. Interplay between Intrinsic Defects, Doping, and Free Carrier Concentration in SrTiO3 Thin Films. Phys. Rev. B: Condens. Matter Mater. Phys. 2012, 85, 195460.

[199] Riedl, H. R.; Schoolar, R. B. Photosensitivity in Epitaxial PbS Films. F. Appl. Phys. 1968, 39, 5086.

[200] Cardona, M.; Greenaway, D. L. Optical Properties and Band Structure of Group IVVI and Group V Materials. Phys. Rev. 1964, 133, A1685.

[201] Yeon, D. H.; Lee, S. M.; Jo, Y. H.; Moon, J.; Cho, Y. S. Origin of the Enhanced Photovoltaic Characteristics of PbS Thin Film Solar Cells Processed at near Room Temperature. F. Mater. Chem. A 2014, 2, 20112.

[202] Mohanty, B. C.; Bector, K.; Laha, R. Elucidating Doping Driven Microstructure Evolution and Optical Properties of Lead Sulfide Thin Films Grown from a Chemical Bath. Appl. Surf. Sci. 2018, 435, 444.

[203] Hone, F. G.; Dejene, F. B. Six Complexing Agents and Their Effects on Optical, Structural, Morphological and Photoluminescence Properties of Lead Sulphide Thin Films Prepared by Chemical Route. f. Lumin. 2018, 201, 321.

[204] Cheraghizade, M.; Jamali-Sheini, F.; Yousefi, R. Optical, Electrical, and Photovoltaic Properties of PbS Thin Films by Anionic and Cationic Dopants. Appl. Phys. A: Mater. Sci. Process. 2017, 123, 1. 
[205] Veena, E.; Bangera, K. V.; Shivakumar, G. K. Effect of Annealing on the Properties of Spray-Pyrolysed Lead Sulphide Thin Films for Solar Cell Application. Appl. Phys. A: Mater. Sci. Process. 2017, 123, 366.

[206] Abbas, M. M.; Shehab, A. A.-M.; Hassan, N.-A.; Al-Samuraee, A.-K. Effect of Temperature and Deposition Time on the Optical Properties of Chemically Deposited Nanostructure PbS Thin Films. Thin Solid Films 2011, 519, 4917.

[207] Azadi Motlagh, Z.; Azim Araghi, M. E. Effect of Annealing Temperature on Optical and Electrical Properties of Lead Sulfide Thin Films. Mater. Sci. Semicond. Process. 2015, 40, 701.

[208] Thangavel, S.; Ganesan, S.; Saravanan, K. Annealing Effect on Cadmium in Situ Doping of Chemical Bath Deposited PbS Thin Films. Thin Solid Films 2012, 520, 5206.

[209] González-Lúa, R.; Escorcia-García, J.; Pérez-Martínez, D.; Nair, M. T. S.; Campos, J.; Nair, P. K. Stable Performance of Chemically Deposited Antimony Sulfide-Lead Sulfide Thin Film Solar Cells under Concentrated Sunlight. ECS 7. Solid State Sci. Technol. 2015, 4, Q9.

[210] Kaci, S.; Keffous, A.; Hakoum, S.; Trari, M.; Mansri, O.; Menari, H. Preparation of Nanostructured PbS Thin Films as Sensing Element for NO2 Gas. Appl. Surf. Sci. 2014, 305, 740 .

[211] Sadovnikov, S. I.; Gusev, A. I. Structure and Properties of PbS Films. F. Alloys Compd. 2013, 573, 65 .

[212] Kotadiya, N. B.; Kothari, A. J.; Tiwari, D.; Chaudhuri, T. K. Photoconducting Nanocrystalline Lead Sulphide Thin Films Obtained by Chemical Bath Deposition. Appl. Phys. A: Mater. Sci. Process. 2012, 108, 819.

[213] Göde, F.; Güneri, E.; Emen, F. M.; Emir Kafadar, V.; Ünlü, S. Synthesis, Structural, Optical, Electrical and Thermoluminescence Properties of Chemically Deposited PbS Thin Films. F. Lumin. 2014, 147, 41.

[214] Silva Filho, J. M. C.; Ermakov, V. A.; Marques, F. C. Perovskite Thin Film Synthesised from Sputtered Lead Sulphide. Sci. Rep. 2018, 8, 1563.

[215] Gibson, A. F. The Absorption Spectra of Solid Lead Sulphide, Selenide and Telluride. Proc. Phys. Soc., London, Sect. B 1950, 63, 756.

[216] Gibson, A. F. The Absorption Spectra of Single Crystals of Lead Sulphide, Selenide and Telluride. Proc. Phys. Soc., London, Sect. B 1952, 65, 378.

[217] Scanlon, W. Solid State Physics; Elsevier, 1959; pp 83-137.

[218] Suram, S. K.; Newhouse, P. F.; Gregoire, J. M. High Throughput Light Absorber Discovery, Part 1: An Algorithm for Automated Tauc Analysis. ACS Comb. Sci. 2016, $18,673$. 
[219] Albanesi, E. A.; Peltzer y Blanca, E. L.; Petukhov, A. G. Calculated Optical Spectra of IV-VI Semiconductors PbS, PbSe and PbTe. Comput. Mater. Sci. 2005, 32, 85.

[220] Ivanov, I. L.; Steparuk, A. S.; Bolyachkina, M. S.; Tsvetkov, D. S.; Safronov, A. P.; Zuev, A. Y. Thermodynamics of Formation of Hybrid Perovskite-Type Methylammonium Lead Halides. F. Chem. Thermodyn. 2018, 116, 253.

[221] Ciccioli, A.; Latini, A. Thermodynamics and the Intrinsic Stability of Lead Halide Perovskites CH3NH3PbX3. F. Phys. Chem. Lett. 2018, 9, 3756.

[222] Hultqvist, A.; Aitola, K.; Sveinbjörnsson, K.; Saki, Z.; Larsson, F.; Törndahl, T.; Johansson, E.; Boschloo, G.; Edoff, M. Atomic Layer Deposition of Electron Selective $\mathrm{SnOx}$ and $\mathrm{ZnO}$ Films on Mixed Halide Perovskite: Compatibility and Performance. ACS Appl. Mater. Interfaces 2017, 9, 29707.

[223] Palmstrom, A. F.; Raiford, J. A.; Prasanna, R.; Bush, K. A.; Sponseller, M.; Cheacharoen, R.; Minichetti, M. C.; Bergsman, D. S.; Leijtens, T.; Wang, H.-P.; Bulović, V.; McGehee, M. D.; Bent, S. F. Interfacial Effects of Tin Oxide Atomic Layer Deposition in Metal Halide Perovskite Photovoltaics. Adv. Energy Mater. 2018, 8, 1800591.

[224] Wang, H.-B.; Ma, D.-Y.; Ma, F.; Xu, K.-W. Impact of Ultrathin Al2O3 Interlayer on Thermal Stability and Leakage Current Properties of TiO2/Al2O3 Stacking Dielectrics. F. Vac. Sci. Technol., B: Nanotechnol. Microelectron.: Mater., Process., Meas., Phenom. 2012, 30, 040601.

[225] Cant, D. J. H.; Syres, K. L.; Lunt, P. J. B.; Radtke, H.; Treacy, J.; Thomas, P. J.; Lewis, E. A.; Haigh, S. J.; O’Brien, P.; Schulte, K.; Bondino, F.; Magnano, E.; Flavell, W. R. Surface Properties of Nanocrystalline PbS Films Deposited at the Water-Oil Interface: A Study of Atmospheric Aging. Langmuir 2015, 31, 1445.

[226] Wilson, C. A.; Grubbs, R. K.; George, S. M. Nucleation and Growth during Al2O3 Atomic Layer Deposition on Polymers. Chem. Mater. 2005, 17, 5625.

[227] Hirvikorpi, T.; Vähä-Nissi, M.; Mustonen, T.; Iiskola, E.; Karppinen, M. Atomic Layer Deposited Aluminum Oxide Barrier Coatings for Packaging Materials. Thin Solid Films 2010, 518, 2654.

[228] Tsai, F. T.; Chao, C. K.; Jhong, K. J.; Chang, R. C. Characterization of Oxide Barrier Layers Prepared by Atomic Layer Deposition. Adv. Mech. Eng. 2017, 9, 1.

[229] Yoon, K. H.; Kim, H.; Koo Lee, Y.-E.; Shrestha, N. K.; Sung, M. M. UV-Enhanced Atomic Layer Deposition of Al2O3 Thin Films at Low Temperature for GasDiffusion Barriers. RSC Adv. 2017, 7, 5601.

[230] Klumbies, H.; Schmidt, P.; Hähnel, M.; Singh, A.; Schroeder, U.; Richter, C.; Mikolajick, T.; Hoßbach, C.; Albert, M.; Bartha, J. W.; Leo, K.; Müller-Meskamp, L. Thickness Dependent Barrier Performance of Permeation Barriers Made from Atomic Layer Deposited Alumina for Organic Devices. Org. Electron. 2015, 17, 138. 
[231] Gynane, M. J. S.; Harris, D. H.; Lappert, M. F.; Power, P. P.; Rivière, P.; RivièreBaudet, M. Subvalent Group 4B Metal Alkyls and Amides. Part 5. The Synthesis and Physical Properties of Thermally Stable Amides of Germanium(II), Tin(II), and Lead(II). F. Chem. Soc., Dalton Trans. 1977, 2004.

[232] Dasgupta, N. P.; Mack, J. F.; Langston, M. C.; Bousetta, A.; Prinz, F. B. Design of an Atomic Layer Deposition Reactor for Hydrogen Sulfide Compatibility. Rev. Sci. Instrum. 2010, 81, 044102.

[233] Nafees, M.; Ikram, M.; Ali, S. Thermal Stability of Lead Sulfide and Lead Oxide Nano-Crystalline Materials. Appl. Nanosci. 2017, 7, 399.

[234] Sadovnikov, S. I.; Kozhevnikova, N. S.; Rempel, A. A. Oxidation of Nanocrystalline Lead Sulfide in Air. Russ. F. Inorg. Chem. 2011, 56, 1864.

[235] Yasaka, M. X-Ray Thin-Film Measurement Techniques V. Rigaku f. 2010, 26, 4.

[236] Langford, J. I.; Wilson, A. J. C. Scherrer after Sixty Years: A Survey and Some New Results in the Determination of Crystallite Size. F. Appl. Crystallogr. 1978, 11, 102.

[237] Neese, F. Software Update: The ORCA Program System, Version 4.0. Wiley Interdiscip. Rev.: Comput. Mol. Sci. 2018, 8, 8.

[238] Najibi, A.; Goerigk, L. The Nonlocal Kernel in van Der Waals Density Functionals as an Additive Correction: An Extensive Analysis with Special Emphasis on the B97M-V and wB97M-V Approaches. f. Chem. Theory Comput. 2018, 14, 5725.

[239] Grimme, S.; Antony, J.; Ehrlich, S.; Krieg, H. A Consistent and Accurate Ab Initio Parametrization of Density Functional Dispersion Correction (DFT-D) for the 94 Elements H-Pu. J. Chem. Phys. 2010, 132, 154104.

[240] Grimme, S.; Ehrlich, S.; Goerigk, L. Effect of the Damping Function in Dispersion Corrected Density Functional Theory. F. Comput. Chem. 2011, 32, 1456.

[241] Weigend, F.; Ahlrichs, R. Balanced basis sets of split valence, triple zeta valence and quadruple zeta valence quality for $\mathrm{H}$ to Rn: Design and assessment of accuracy. Physical Chemistry Chemical Physics 2005, 7, 3297.

[242] Metz, B.; Stoll, H.; Dolg, M. Small-Core Multiconfiguration-Dirac-Hartree-FockAdjusted Pseudopotentials for Post- d Main Group Elements: Application to $\mathrm{PbH}$ and PbO. F. Chem. Phys. 2000, 113, 2563.

[243] Neese, F.; Wennmohs, F.; Hansen, A.; Becker, U. Efficient, Approximate and Parallel Hartree-Fock and Hybrid DFT Calculations. A 'Chain-of-Spheres' Algorithm for the Hartree-Fock Exchange. Chem. Phys. 2009, 356, 98.

[244] Weigend, F. Accurate Coulomb-fitting basis sets for H to Rn. Physical Chemistry Chemical Physics 2006, 8, 1057. 
[245] Leskelä, M.; Niinistö, L.; Niemela, P.; Nykänen, E.; Soininen, P.; Tiitta, M.; Vähäkangas, J. Preparation of lead sulfide thin films by the atomic layer epitaxy process. Vacuum 1990, 41, 1457-1459.

[246] Lee, W.; Dasgupta, N. P.; Trejo, O.; Lee, J.-R.; Hwang, J.; Usui, T.; Prinz, F. B. AreaSelective Atomic Layer Deposition of Lead Sulfide: Nanoscale Patterning and DFT Simulations. Langmuir 2010, 26, 6845-6852.

[247] Watanabe, T.; Hoffmann-Eifert, S.; Mi, S.; Jia, C.; Waser, R.; Hwang, C. S. Growth of ternary $\mathrm{PbTiOx}$ films in a combination of binary oxide atomic layer depositions. fournal of Applied Physics 2007, 101, 014114.

[248] Harjuoja, J.; Putkonen, M.; Niinistö, L. Exploiting volatile lead compounds as precursors for the atomic layer deposition of lead dioxide thin films. Thin Solid Films 2006, 497, 77-82.

[249] Zhang, K.; Pillai, A. D. R.; Bollenbach, K.; Nminibapiel, D.; Cao, W.; Baumgart, H.; Scherer, T.; Chakravadhanula, V. S. K.; Kübel, C.; Kochergin, V. Atomic Layer Deposition of Nanolaminate Structures of Alternating PbTe and PbSe Thermoelectric Films. ECS Journal of Solid State Science and Technology 2014, 3, P207-P212.

[250] Lee, H. J.; Kim, G. H.; Lee, K.; Hwang, C. S. Initial Growth Behavior of a Lead Oxide Thin Film on Ir Substrates by Atomic Layer Deposition. Electrochemical and SolidState Letters 2007, 10, G89.

[251] Hwang, G. W.; Lee, H. J.; Lee, K.; Hwang, C. S. Atomic Layer Deposition and Electrical Properties of PbTiO[sub 3] Thin Films Using Metallorganic Precursors and $\mathrm{H}$ [sub 2]O. Journal of The Electrochemical Society 2007, 154, G69.

[252] Lee, H. J.; Park, M. H.; Min, Y.-S.; Clavel, G.; Pinna, N.; Hwang, C. S. Unusual Growth Behavior of Atomic Layer Deposited PbTiO3 Thin Films Using Water and Ozone As Oxygen Sources and Their Combination. The fournal of Physical Chemistry C 2010, $114,12736-12741$.

[253] OTANI, Y.; UCHIYAMA, K.; OKAMURA, S.; SHIOSAKI, T. LOW TEMPERATURE DEPOSITION OF Pb(Zr,Ti)O3 THIN FILMS BY LIQUID DELIVERY MOCVD USING A COCKTAIL SOURCE WITH Pb(DMAMP)2, Zr(MMP)4 AND Ti(MMP)4. Integrated Ferroelectrics 2006, 81, 261-270.

[254] Strnad, N. A.; Hanrahan, B. M.; Potrepka, D. M.; Pulskamp, J. S.; Phaneuf, R. J.; Polcawich, R. G. Growth of thin film ferroelectric PZT, PHT, and antiferroelectric PHO from atomic layer deposition precursors. Fournal of the American Ceramic Society $n / a$.

[255] Sbrockey, N. M.; Tompa, G. S.; Lavelle, R.; Trumbull, K. A.; Fanton, M. A.; Snyder, D. W.; Polcawich, R. G.; Potrepka, D. M. Atomic layer deposition of PbTiO3 and PbZrxTi1-xO3 films using metal alkyl and alkylamide precursors. Fournal of Vacuum Science \& Technology A 2018, 36, 031509. 
[256] Popov, G. et al. Atomic Layer Deposition of PbS Thin Films at Low Temperatures. Chemistry of Materials 2020, 32, 8216-8228.

[257] Li, Z.; Barry, S. T.; Gordon, R. G. Synthesis and Characterization of Copper(I) Amidinates as Precursors for Atomic Layer Deposition (ALD) of Copper Metal. Inorganic Chemistry 2005, 44, 1728-1735, PMID: 15762699.

[258] Hausmann, D. M.; Kim, E.; Becker, J.; Gordon, R. G. Atomic Layer Deposition of Hafnium and Zirconium Oxides Using Metal Amide Precursors. Chemistry of Materials 2002, 14, 4350-4358.

[259] Gordon, R. G.; Lim, B. S. Atomic layer deposition using metal amidinates. 2009; US Patent 7,557,229.

[260] Veith, M.; Grosser, M. Cyclische Diazastannylene, XVI [1]. Ringe und Käfige mit $\mathrm{Ge}(\mathrm{II}), \mathrm{Sn}(\mathrm{II})$ und $\mathrm{Pb}(\mathrm{II})$ / Cyclic Diazastannylenes, XVI [1]. Rings and Cages with $\mathrm{Ge}(\mathrm{II}), \mathrm{Sn}(\mathrm{II})$ and $\mathrm{Pb}(\mathrm{II})$. Zeitschrift für Naturforschung B 01 Nov. 1982, 37, 1375 1381.

[261] Veith, M.; Olbrich, M.; Shihua, W.; Huch, V. Tin-carbon bond formation by simple addition of cyclic dienes to a bis(amino)stannylene: a mechanistic study. F. Chem. Soc., Dalton Trans. 1996, 161-165.

[262] Veith, M.; Hans, J.; Stahl, L.; May, P.; Huch, V.; Sebald, A. Alkoxigermanate(II), -stannate(II) und -plumbate(II) zweiwertiger Metallionen, I [1] / Alkoxigermanates(II), -stannates(II) and -plumbates(II) of Two-Valent Metal Ions, I [1]. Zeitschrift für Naturforschung B 1991, 46, 403-424.

[263] Papiernik, R.; Hubert-Pfalzgrl, L. G.; Veith, M.; Huch, V. Complexes of Early Transition Metal Alkoxides: Molecular Structure of Ti(OiPr)4[OPb4(tBuN)3]. Chemische Berichte 1997, 130, 1361-1363.

[264] Veith, M.; Grosser, M.; Huch, V. Cyclische Diazastannylene. XIX. Zur Reaktion eines Bis(amino)germylens, -stannylens und -plumbylens mit Phosphortrichlorid und 2, 3-Dimethyl-1, 3-butadien. Zeitschrift für anorganische und allgemeine Chemie 1984, 513, 89-102.

[265] Schranz, I.; Lief, G. R.; Midstokke, S. J.; Stahl, L. Inorganic Heterocyclic Bis(phosphines): Syntheses and Structures of a 1,2Bis(diazasilaphosphetidino)ethane and Its Nickel, Molybdenum, and Rhodium Complexes. Inorganic Chemistry 2002, 41, 6919-6927, PMID: 12470091.

[266] Wrackmeyer, B.; Pedall, A.; Weidinger, J. N-Silylaminotin Trichlorides. Synthesis and Characterisation by Multinuclear Magnetic Resonance Spectroscopy. Zeitschrift für Naturforschung B 01 Oct. 2001, 56, 1009 - 1014.

[267] Veith, M.; Frères, J.; König, P.; Schütt, O.; Huch, V.; Blin, J. Nanoscaled Sn and Pb Particles Aligned in Al2O3 Tubes Obtained from Molecular Precursors. European fournal of Inorganic Chemistry 2005, 2005, 3699-3710. 
[268] Kim, S.-J.; Lee, Y.-J.; Kim, S. H.; Ko, J.; Cho, S.; Kang, S. O. Syntheses, Structural Characterizations, and Metathesis Studies of New Dimeric Group 14 Metal Complexes Derived from Silacycloalkyl Diamide. Organometallics 2002, 21, 5358-5365.

[269] Yang, D.; Guo, J.; Wu, H.; Ding, Y.; Zheng, W. Synthesis and structural characterization of two-coordinate low-valent 14-group metal complexes bearing bulky bis(amido)silane ligands. Dalton Transactions 2012, 41, 2187.

[270] Stader, C.; Wrackmeyer, B. Natural abundance 15N nuclear magnetic resonance of monomeric cyclic and noncyclic tin(II) and lead(II) amides. Fournal of Magnetic Resonance (1969) 1987, 72, $544-547$.

[271] Wrackmeyer, B.; Stader, C.; Horchler, K. Multinuclear magnetic resonance studies of cyclic monomeric bis(amino)plumbylenes and bis(amino)stannylenes and a new type of correlation between $119 \mathrm{Sn}$ and $207 \mathrm{~Pb}$ chemical shifts. Fournal of Magnetic Resonance (1969) 1989, 83, 601 - 607.

[272] Andreocci, M. V.; Cauletti, C.; Stranges, S.; Wrackmeyer, B.; Stader, C. UV Photoelectron Spectra and Pseudopotential "ab initio" Calculations of Some 4-Membered Cyclic Amides of Group XIV Elements. Zeitschrift für Naturforschung B 01 Jan. 1991, 46, $39-46$.

[273] Smith, M. B. The monomer-dimer equilibria of liquid aliminum alkyls. Fournal of Organometallic Chemistry 1972, 46, 31-49.

[274] Ehm, C.; Cipullo, R.; Budzelaar, P. H. M.; Busico, V. Role(s) of TMA in polymerization. Dalton Transactions 2016, 45, 6847-6855.

[275] Adomaitis, R. A. Estimating the thermochemical properties of trimethylaluminum for thin-film processing applications. Journal of Vacuum Science \& Technology A 2018, 36, 050602.

[276] Johnson, E. R.; Keinan, S.; Mori-Sańchez, P.; Contreras-Garciá, J.; Cohen, A. J.; Yang, W. Revealing Noncovalent Interactions. Journal of the American Chemical Society 2010, 132, 6498-6506.

[277] Banert, K.; Heck, M.; Ihle, A.; Kronawitt, J.; Pester, T.; Shoker, T. Steric Hindrance Underestimated: It is a Long, Long Way to Tri-tert-alkylamines. The fournal of Organic Chemistry 2018, 83, 5138-5148.

[278] Banert, K.; Seifert, J. Steric hindrance classified: treatment of isothiocyanatoallene with secondary amines bearing bulky substituents to generate 2-aminothiazoles. Organic Chemistry Frontiers 2019, 6, 3517-3522.

[279] Boysen, N.; Hasselmann, T.; Karle, S.; Rogalla, D.; Theirich, D.; Winter, M.; Riedl, T.; Devi, A. An N-Heterocyclic Carbene Based Silver Precursor for Plasma-Enhanced Spatial Atomic Layer Deposition of Silver Thin Films at Atmospheric Pressure. Angewandte Chemie International Edition 2018, 57, 16224-16227. 
[280] Johnson, H. G. Reaction of Aliphatic Amines with Formaldehyde and Nitroparaffins. II. Secondary Amines. Fournal of the American Chemical Society 1946, 68, 12-14.

[281] Noble, A.; Anderson, J. C. Nitro-Mannich Reaction. Chemical Reviews 2013, 113, 2887-2939.

[282] Murray, J. S.; Politzer, P. Statistical analysis of the molecular surface electrostatic potential: an approach to describing noncovalent interactions in condensed phases. fournal of Molecular Structure: THEOCHEM 1998, 425, 107-114.

[283] Politzer, P.; Murray, J. S. The fundamental nature and role of the electrostatic potential in atoms and molecules. Theoretical Chemistry Accounts: Theory, Computation, and Modeling (Theoretica Chimica Acta) 2002, 108, 134-142.

[284] Clark, T. QSAR and QSPR based solely on surface properties? Journal of Molecular Graphics and Modelling 2004, 22, 519-525.

[285] Naray-Szabo, G.; Ferenczy, G. G. Molecular Electrostatics. Chemical Reviews 1995, 95, 829-847.

[286] Murray, J. S.; Lane, P.; Brinck, T.; Paulsen, K.; Grice, M. E.; Politzer, P. Relationships of critical constants and boiling points to computed molecular surface properties. The Journal of Physical Chemistry 1993, 97, 9369-9373.

[287] Wang, B. et al. Synthesis and reactivity of a mononuclear non-haem cobalt(IV)-oxo complex. Nature Communications 2017, 8 .

[288] O'Neill, M. L.; Bowen, H. R.; Derecskei-Kovacs, A.; Cuthill, K. S.; Han, B.; Xiao, M. Impact of Aminosilane Precursor Structure on Silicon Oxides by Atomic Layer Deposition. Interface magazine 2011, 20, 33-37.

[289] Passarelli, V.; Benetollo, F.; Zanella, P.; Carta, G.; Rossetto, G. Synthesis and characterisation of novel zirconium(iv) derivatives containing the bis-amido ligand SiMe2(NRR/)2. Dalton Transactions 2003, 1411-1418.

[290] Horton, A. D.; de With, J. Controlled Alkene and Alkyne Insertion Reactivity of a Cationic Zirconium Complex Stabilized by an Open Diamide Ligand. Organometallics 1997, 16, 5424-5436.

[291] Kuhn, N.; Kratz, T. Synthesis of Imidazol-2-ylidenes by Reduction of Imidazole2(3H)-thiones. Synthesis 1993, 1993, 561-562.

[292] Zhang, Y.; Yang, W. Comment on "Generalized Gradient Approximation Made Simple”. Physical Review Letters 1998, 80, 890-890.

[293] van Lenthe, E.; Baerends, E. J.; Snijders, J. G. Relativistic total energy using regular approximations. The fournal of Chemical Physics 1994, 101, 9783-9792.

[294] van Lenthe, E.; Baerends, E. J.; Snijders, J. G. Relativistic regular two-component Hamiltonians. The fournal of Chemical Physics 1993, 99, 4597-4610. 
[295] van Lenthe, E.; Ehlers, A.; Baerends, E.-J. Geometry optimizations in the zero order regular approximation for relativistic effects. The fournal of Chemical Physics 1999, 110, 8943-8953.

[296] Pantazis, D. A.; Neese, F. All-electron scalar relativistic basis sets for the 6p elements. Theoretical Chemistry Accounts 2012, 131.

[297] Zhao, Y.; Truhlar, D. G. Design of Density Functionals That Are Broadly Accurate for Thermochemistry, Thermochemical Kinetics, and Nonbonded Interactions. The Journal of Physical Chemistry A 2005, 109, 5656-5667.

[298] Riplinger, C.; Sandhoefer, B.; Hansen, A.; Neese, F. Natural triple excitations in local coupled cluster calculations with pair natural orbitals. The fournal of Chemical Physics 2013, 139, 134101.

[299] Lu, T.; Chen, F. Multiwfn: A multifunctional wavefunction analyzer. fournal of Computational Chemistry 2011, 33, 580-592.

[300] Mayer, I. Bond order and valence indices: A personal account. fournal of Computational Chemistry 2006, 28, 204-221.

[301] Bader, R. F. W.; Beddall, P. M. Virial Field Relationship for Molecular Charge Distributions and the Spatial Partitioning of Molecular Properties. The fournal of Chemical Physics 1972, 56, 3320-3329.

[302] Bader, R. F. W. Everyman's Derivation of the Theory of Atoms in Molecules. The Journal of Physical Chemistry A 2007, 111, 7966-7972.

[303] Lee, P.-S.; Fujita, T.; Yoshikai, N. Cobalt-Catalyzed, Room-Temperature Addition of Aromatic Imines to Alkynes via Directed $\mathrm{C}-\mathrm{H}$ Bond Activation. Fournal of the American Chemical Society 2011, 133, 17283-17295.

[304] Gao, K.; Yoshikai, N. Cobalt-Phenanthroline Catalysts for the ortho Alkylation of Aromatic Imines under Mild Reaction Conditions. Angewandte Chemie International Edition 2011, 50, 6888-6892.

[305] Moselage, M.; Sauermann, N.; Richter, S. C.; Ackermann, L. C-H Alkenylations with Alkenyl Acetates, Phosphates, Carbonates, and Carbamates by Cobalt Catalysis at $23^{\circ} \mathrm{C}$. Angewandte Chemie International Edition 2015, 54, 6352-6355.

[306] Song, W.; Ackermann, L. Cobalt-Catalyzed Direct Arylation and Benzylation by $\mathrm{C}-\mathrm{H} / \mathrm{C}-\mathrm{O}$ Cleavage with Sulfamates, Carbamates, and Phosphates. Angewandte Chemie International Edition 2012, 51, 8251-8254.

[307] Obligacion, J. V.; Semproni, S. P.; Chirik, P. J. Cobalt-Catalyzed C-H Borylation. Journal of the American Chemical Society 2014, 136, 4133-4136.

[308] Gao, K.; Yoshikai, N. Cobalt-Catalyzed Ortho Alkylation of Aromatic Imines with Primary and Secondary Alkyl Halides. Fournal of the American Chemical Society 2013, 135, 9279-9282. 
[309] Bellow, J. A.; Stoian, S. A.; van Tol, J.; Ozarowski, A.; Lord, R. L.; Groysman, S. Synthesis and Characterization of a Stable High-Valent Cobalt Carbene Complex. Journal of the American Chemical Society 2016, 138, 5531-5534.

[310] Karppinen, M.; Asako, I.; Motohashi, T.; Yamauchi, H. Oxidation State of Cobalt in the $\mathrm{NaxCoO} 2-\delta \cdot y H 2 \mathrm{O}$ Superconductor. Chemistry of Materials 2004, 16, 1693-1696.

[311] Motohashi, T.; Ono, T.; Sugimoto, Y.; Masubuchi, Y.; Kikkawa, S.; Kanno, R.; Karppinen, M.; Yamauchi, H. Electronic phase diagram of the layered cobalt oxide systemLixCoO2(0.0 $\leq x \leq 1.0)$. Physical Review B 2009, 80 .

[312] Li, H.; Gao, Y.; Shao, Y.; Su, Y.; Wang, X. Vapor-Phase Atomic Layer Deposition of Co9S8 and Its Application for Supercapacitors. Nano Letters 2015, 15, 6689-6695.

[313] Kim, J.; Iivonen, T.; Hämäläinen, J.; Kemell, M.; Meinander, K.; Mizohata, K.; Wang, L.; Räisänen, J.; Beranek, R.; Leskelä, M.; Devi, A. Low-Temperature Atomic Layer Deposition of Cobalt Oxide as an Effective Catalyst for Photoelectrochemical Water-Splitting Devices. Chemistry of Materials 2017, 29, 5796-5805.

[314] Kim, D.; Song, J.-G.; Yang, H.; Lee, H.; Park, J.; Kim, H. Textile-based highperformance hydrogen evolution of low-temperature atomic layer deposition of cobalt sulfide. Nanoscale 2019, 11, 844-850.

[315] Higgins, D. C.; Hassan, F. M.; Seo, M. H.; Choi, J. Y.; Hoque, M. A.; Lee, D. U.; Chen, Z. Shape-controlled octahedral cobalt disulfide nanoparticles supported on nitrogen and sulfur-doped graphene/carbon nanotube composites for oxygen reduction in acidic electrolyte. Fournal of Materials Chemistry A 2015, 3, 6340-6350.

[316] Kaloyeros, A. E.; Pan, Y.; Goff, J.; Arkles, B. Editors' Choice-Review-Cobalt Thin Films: Trends in Processing Technologies and Emerging Applications. ECS fournal of Solid State Science and Technology 2019, 8, P119-P152.

[317] Collins, T. J.; Powell, R. D.; Slebodnick, C.; Uffelman, E. S. Stable highly oxidizing cobalt complexes of macrocyclic ligands. Fournal of the American Chemical Society 1991, 113, 8419-8425.

[318] Topich, J.; Halpern, J. Organobis(dioximato)cobalt(IV) complexes: electron paramagnetic resonance spectra and electronic structures. Inorganic Chemistry 1979, 18, 1339-1343.

[319] Will, S.; Lex, J.; Vogel, E.; Adamian, V. A.; Caemelbecke, E. V.; Kadish, K. M. Synthesis, Characterization, and Electrochemistry of $\sigma$-Bonded Cobalt Corroles in High Oxidation States. Inorganic Chemistry 1996, 35, 5577-5583.

[320] Zhang, L.; Liu, Y.; Deng, L. Three-Coordinate Cobalt(IV) and Cobalt(V) Imido Complexes with N-Heterocyclic Carbene Ligation: Synthesis, Structure, and Their Distinct Reactivity in $\mathrm{C}-\mathrm{H}$ Bond Amination. Journal of the American Chemical Society 2014, 136, 15525-15528. 
[321] Lewis, R. A.; George, S. P.; Chapovetsky, A.; Wu, G.; Figueroa, J. S.; Hayton, T. W. Synthesis of a cobalt(iv) ketimide with a squashed tetrahedral geometry. Chemical Communications 2013, 49, 2888.

[322] Byrne, E. K.; Richeson, D. S.; Theopold, K. H. Tetrakis(1-norbornyl)cobalt, a low spin tetrahedral complex of a first row transition metal. Fournal of the Chemical Society, Chemical Communications 1986, 1491.

[323] Bower, B. K.; Tennent, H. G. Transition metal bicyclo[2.2.1]hept-1-yls. fournal of the American Chemical Society 1972, 94, 2512-2514.

[324] Veith, M. Cyclische Stickstoffderivat des vier- und zweiwertigen Zinns. Angewandte Chemie 1975, 87, 287-288.

[325] Lappert, M.; Protchenko, A.; Power, P. P.; Seeber, A. Metal amide chemistry; John Wiley \& Sons, 2008.

[326] Yang, L.; Powell, D. R.; Houser, R. P. Structural variation in copper(i) complexes with pyridylmethylamide ligands: structural analysis with a new four-coordinate geometry index, $\tau 4$. Dalton Trans. 2007, 955-964.

[327] Brown, S. D.; Betley, T. A.; Peters, J. C. A Low-Spin d5Iron Imide: Nitrene Capture by Low-Coordinate Iron(I) Provides the 4-Coordinate Fe(III) Complex [PhB(CH2PPh2)3]Fe:N-p-tolyl. Journal of the American Chemical Society 2003, 125 , 322-323.

[328] Swart, M.; Gruden, M. Spinning around in Transition-Metal Chemistry. Accounts of Chemical Research 2016, 49, 2690-2697.

[329] Dasgupta, N. P.; Meng, X.; Elam, J. W.; Martinson, A. B. F. Atomic Layer Deposition of Metal Sulfide Materials. Accounts of Chemical Research 2015, 48, 341-348.

[330] Väyrynen, K.; Hatanpää, T.; Mattinen, M.; Heikkilä, M.; Mizohata, K.; Meinander, K.; Räisänen, J.; Ritala, M.; Leskelä, M. Diamine Adduct of Cobalt(II) Chloride as a Precursor for Atomic Layer Deposition of Stoichiometric Cobalt(II) Oxide and Reduction Thereof to Cobalt Metal Thin Films. Chemistry of Materials 2018, 30, 34993507.

[331] Kerrigan, M. M.; Klesko, J. P.; Winter, C. H. Low Temperature, Selective Atomic Layer Deposition of Cobalt Metal Films Using Bis(1, 4-di-tert-butyl-1, 3diazadienyl)cobalt and Alkylamine Precursors. Chemistry of Materials 2017, 29, 7458-7466.

[332] Land, M. A.; Robertson, K. N.; Barry, S. T. Ligand-Assisted Volatilization and Thermal Stability of Bis(imido)dichloromolybdenum(VI) $([(\mathrm{t}-\mathrm{BuN}=) 2 \mathrm{MoCl} 2] 2)$ and Its Adducts. Organometallics 2019, 39, 916-927.

[333] Leskelä, M.; Ritala, M. Atomic layer deposition (ALD): from precursors to thin film structures. Thin Solid Films 2002, 409, 138-146. 
[334] Sacconi, L.; Bertini, I.; Mani, F. Metal complexes of N, N, N', N'-tetramethyldiamines. I. Nickel(II) and cobalt(II) complexes. Inorganic Chemistry 1967, 6, 262-267.

[335] Knisley, T. J.; Saly, M. J.; Heeg, M. J.; Roberts, J. L.; Winter, C. H. Volatility and High Thermal Stability in Mid- to Late-First-Row Transition-Metal Diazadienyl Complexes. Organometallics 2011, 30, 5010-5017.

[336] Sheldrick, G. M. SHELXT- Integrated space-group and crystal-structure determination. Acta Crystallographica Section A Foundations and Advances 2015, 71, 3-8.

[337] Sheldrick, G. M. Crystal structure refinement withSHELXL. Acta Crystallographica Section C Structural Chemistry 2015, 71, 3-8.

[338] Hübschle, C. B.; Sheldrick, G. M.; Dittrich, B. ShelXle: a Qt graphical user interface forSHELXL. Fournal of Applied Crystallography 2011, 44, 1281-1284.

[339] Dolomanov, O. V.; Bourhis, L. J.; Gildea, R. J.; Howard, J. A. K.; Puschmann, H. OLEX2: a complete structure solution, refinement and analysis program. fournal of Applied Crystallography 2009, 42, 339-341.

[340] Sauerbrey, G. Verwendung von Schwingquarzen zur Wügung dünner Schichten und zur Mikrowägung. Zeitschrift für Physik 1959, 155, 206-222.

[341] Frisch, M. J. et al. Gaussian 16 Revision C.01. 2016; Gaussian Inc. Wallingford CT.

[342] Perdew, J. P.; Burke, K.; Ernzerhof, M. Generalized Gradient Approximation Made Simple. Physical Review Letters 1996, 77, 3865-3868.

[343] Austin, A.; Petersson, G. A.; Frisch, M. J.; Dobek, F. J.; Scalmani, G.; Throssell, K. A Density Functional with Spherical Atom Dispersion Terms. Fournal of Chemical Theory and Computation 2012, 8, 4989-5007.

[344] Becke, A. D. Density-functional thermochemistry. V. Systematic optimization of exchange-correlation functionals. The fournal of Chemical Physics 1997, 107, 85548560 .

[345] Chai, J.-D.; Head-Gordon, M. Long-range corrected hybrid density functionals with damped atom-atom dispersion corrections. Physical Chemistry Chemical Physics 2008, 10, 6615 .

[346] Neese, F. Software update: the ORCA program system, version 4.0. WIREs Computational Molecular Science 2017, 8.

[347] Becke, A. D. Density-functional exchange-energy approximation with correct asymptotic behavior. Physical Review A 1988, 38, 3098-3100.

[348] Lee, C.; Yang, W.; Parr, R. G. Development of the Colle-Salvetti correlation-energy formula into a functional of the electron density. Physical Review B 1988, 37, 785789. 
[349] HANDY, N. C.; COHEN, A. J. Left-right correlation energy. Molecular Physics 2001, 99, 403-412.

[350] Hoe, W.-M.; Cohen, A. J.; Handy, N. C. Assessment of a new local exchange functional OPTX. Chemical Physics Letters 2001, 341, 319-328.

[351] Swart, M.; Groenhof, A. R.; Ehlers, A. W.; Lammertsma, K. Validation of ExchangeCorrelation Functionals for Spin States of Iron Complexes. The fournal of Physical Chemistry A 2004, 108, 5479-5483.

[352] Neese, F.; Wennmohs, F.; Hansen, A.; Becker, U. Efficient, approximate and parallel Hartree-Fock and hybrid DFT calculations. A 'chain-of-spheres' algorithm for the Hartree-Fock exchange. Chemical Physics 2009, 356, 98-109.

[353] Neese, F. Importance of Direct Spin-Spin Coupling and Spin-Flip Excitations for the Zero-Field Splittings of Transition Metal Complexes: A Case Study. Fournal of the American Chemical Society 2006, 128, 10213-10222.

[354] Humphrey, W.; Dalke, A.; Schulten, K. VMD: Visual molecular dynamics. fournal of Molecular Graphics 1996, 14, 33-38.

[355] Ellmer, K. Past Achievements and Future Challenges in the Development of Optically Transparent Electrodes. Nat. Photonics 2012, 6, 809.

[356] Gordon, R. G. Criteria for Choosing Transparent Conductors. MRS Bull. 2000, 25, 52.

[357] Andersson, A.; Johansson, N.; Bröms, P.; Yu, N.; Lupo, D.; Salaneck, W. R. Fluorine Tin Oxide as an Alternative to Indium Tin Oxide in Polymer LEDs. Adv. Mater. 1998, 10, 859 .

[358] Choi, Y.-J.; Park, H.-H. A Simple Approach to the Fabrication of Fluorine-Doped Zinc Oxide Thin Films by Atomic Layer Deposition at Low Temperatures and an Investigation into the Growth Mode. F. Mater. Chem. C 2014, 2, 98.

[359] Lee, Y.; Sun, H.; Young, M. J.; George, S. M. Atomic Layer Deposition of Metal Fluorides Using HF-Pyridine as the Fluorine Precursor. Chem. Mater. 2016, 28, 2022.

[360] Pilvi, T.; Puukilainen, E.; Kreissig, U.; Leskelä, M.; Ritala, M. Atomic Layer Deposition of MgF2 Thin Films Using TaF5 as a Novel Fluorine Source. Chem. Mater. 2008, $20,5023$.

[361] Putkonen, M.; Szeghalmi, A.; Pippel, E.; Knez, M. Atomic Layer Deposition of Metal Fluorides through Oxide Chemistry. F. Mater. Chem. 2011, 21, 14461.

[362] Boutonnet, J. C.; Bingham, P.; Calamari, D.; Rooij, C. d.; Franklin, J.; Kawano, T.; Libre, J.-M.; McCul-loch, A.; Malinverno, G.; Odom, J. M. Environmental Risk Assessment of Trifluoroacetic Acid. Hum. Ecol. Risk Assess. 1999, 5, 59. 
[363] Maruyama, T.; Tabata, K. Fluorine-Doped Tin Dioxide Thin Films Prepared by Chemical Vapor Deposition. F. Appl. Phys. 1990, 68, 4282.

[364] Korotkov, R. Y.; Ricou, P.; Farran, A. J. E. Preferred Orientations in Polycrystalline SnO2 Films Grown by Atmospheric Pressure Chemical Vapor Deposition. Thin Solid Films 2006, 502, 79.

[365] Belanger, D.; Dodelet, J. P.; Lombos, B. A.; Dickson, J. I. Thickness Dependence of Transport Properties of Doped Polycrystalline Tin Oxide Films. F. Electrochem. Soc. 1985, 132, 1398.

[366] Birchall, T.; Faggiani, R.; Lock, C. J. L.; Manivannan, V. Preparation and Partial Oxidation of Tin (II) Trifluoroacetate: Spectroscopic Properties and X-Ray Crystal Structure of Di- $\mu_{3}$-Oxo-Octakis- $\mu$-Trifluoroacetato-Tetratin (II) Tin (IV). F. Chem. Soc., Dalton Trans. 1987, 1675.

[367] Batchelor, R. J.; Birchall, T.; Johnson, J. P. The Crystal Structure of $\mathrm{Sn}_{6} \mathrm{O}_{2}\left(\mathrm{CF}_{3} \mathrm{CO}_{2}\right)_{8} \cdot \mathrm{CF}_{3} \mathrm{CO}_{2}$ H. Can. J. Chem. 1987, 65, 2187.

[368] Birchall, T.; Johnson, J. P. Crystal Structure of Di- $\mu_{3}$-Oxo-Octakis- $\mu-$ (Trifluoroacetato)-Ditin (II) Ditin (IV)-Benzene (1/1). J. Chem. Soc., Dalton Trans. 1981, 69.

[369] Zabula, A. V.; Filatov, A. S.; Petrukhina, M. A. Coordinatively Unsaturated Polynuclear Mixed-Valent SnII-SnIV and CuII-SnIV Oxo-Centered Carboxylates. F. Cluster Sci. 2010, 21, 361.

[370] Daly, S. R.; Kim, D. Y.; Girolami, G. S. Lanthanide N,N-Dimethylaminodiboranates as a New Class of Highly Volatile Chemical Vapor Deposition Precursors. Inorg. Chem. 2012, 51, 7050 .

[371] Cheng, Y.; Emge, T. J.; Brennan, J. G. Polymeric Cd(Se-2-NC5H4)2 and Square Planar $\mathrm{Hg}(\mathrm{Se}-2-\mathrm{NC} 5 \mathrm{H} 4) 2$ : Volatile CVD Precursors to II-VI Semiconductors. Inorg. Chem. 1994, 33, 3711.

[372] Donaldson, J. D.; Moser, W. 789. Pure Tin (II) Sulphate. F. Chem. Soc. 1960, 4000.

[373] Anastas, P. T.; Zimmerman, J. B. Peer Reviewed: Design Through the 12 Principles of Green Engineering. Environ. Sci. Technol. 2003, 37, 94A.

[374] Gao, Y.; Yang, Y.; Zheng, W.; Su, Y.; Zhang, X.; Roesky, H. W. Germanium and Tin Monoxides Trapped by Oxophilic Germylene and Stannylene Ligands. Inorg. Chem. 2017, 56, 10220.

[375] Weimer, M. S.; Hu, B.; Kraft, S. J.; Gordon, R. G.; Segre, C. U.; Hock, A. S. Synthetic and Spectroscopic Study of the Mechanism of Atomic Layer Deposition of Tin Dioxide. Organometallics 2016, 35, 1202. 
[376] Mackus, A. J. M.; MacIsaac, C.; Kim, W.-H.; Bent, S. F. Incomplete Elimination of Precursor Ligands during Atomic Layer Deposition of Zinc-Oxide, Tin-Oxide, and Zinc-Tin-Oxide. F. Chem. Phys. 2017, 146, 052802.

[377] Stafeeva, V. S.; Mitiaev, A. S.; Abakumov, A. M.; Tsirlin, A. A.; Makarevich, A. M.; Antipov, E. V. Crystal Structure and Chemical Bonding in Tin (II) Acetate. Polyhedron 2007, 26, 5365.

[378] Smart, B. A.; Griffiths, L. E.; Pulham, C. R.; Robertson, H. E.; Mitzel, N. W.; Rankin, D. W. H. Molecular Structure of Tin (II) Acetate as Determined in the Gas Phase by Electron Diffraction and Ab Initio Calculations. F. Chem. Soc., Dalton Trans. 1997, 1565.

[379] Alcock, N. W.; Tracy, V. L. Acetates and Acetato Complexes. IV. The Crystal and Molecular Structure of Tin Tetraacetate. Acta Crystallogr., Sect. B: Struct. Crystallogr. Cryst. Chem. 1979, 35, 80.

[380] Donaldson, J. D.; Moser, W.; Simpson, W. B. 1147. Tin (II) Acetates. F. Chem. Soc. 1964, 5942.

[381] Sartori, P.; Weidenbruch, M. Trifluoroacetates of Silicon, Germanium, and Tin. Angew. Chem., Int. Ed. Engl. 1965, 4, 1079.

[382] Sartori, P.; Weidenbruch, M. Über Die Darstellung Und Eigenschaften von Perfluoracyloxy-Verbindungen Der Vierten Gruppe Des Periodensystems. Chem. Ber. 1967, 100, 2049.

[383] Sawyer, A. K.; Frey, C. A Simple Synthesis of Tin(IV) Acetate from Tetraphenyltin. Synth. React. Inorg. Met.-Org. Chem. 1983, 13, 259.

[384] Janssen, D. E.; Wilson, C. V. 4-Iodoveratrole. Org. Synth. 1963, 36, 46.

[385] Rees, W. S.; Carris, M. W.; Girolami, G. S.; You, Y. Bis(2,2,6,6-Tetramethyl-3,5Heptanedionato) Copper. Inorg. Synth. 2007, 286.

[386] Cotton, F. A.; Dikarev, E. V.; Petrukhina, M. A. Syntheses and Crystal Structures of "Unligated" Copper(I) and Copper(II) Trifluoroacetates. Inorg. Chem. 2000, 39, 6072.

[387] Birchall, T.; Johnson, J. P. Preparation and Characterization of Mixed-OxidationState Tin Carboxylates and Related Tin (IV) Carboxylates. Inorg. Chem. 1982, 21, 3724 .

[388] Colominas, C.; Lau, K. H.; Hildenbrand, D. L.; Crouch-Baker, S.; Sanjurjo, A. Vapor Pressures of the Copper and Yttrium $\beta$-Diketonate MOCVD Precursors. Fournal of Chemical \& Engineering Data 2001, 46, 446-450.

[389] Sheldrick, G. M. SHELXT-Integrated Space-Group and Crystal-Structure Determination. Acta Crystallogr., Sect. A: Found. Adv. 2015, 71, 3. 
[390] Hübschle, C. B.; Sheldrick, G. M.; Dittrich, B. ShelXle: A Qt Graphical User Interface for SHELXL. f. Appl. Crystallogr. 2011, 44, 1281.

[391] Dolomanov, O. V.; Bourhis, L. J.; Gildea, R. J.; Howard, J. A. K.; Puschmann, H. OLEX2: A Complete Structure Solution, Refinement and Analysis Program. 7. Appl. Crystallogr. 2009, 42, 339.

[392] Frisch, M. J. et al. Gaussian09 Revision D.01. Gaussian Inc. Wallingford CT 2009.

[393] Douglas, M.; Kroll, N. M. Quantum Electrodynamical Corrections to the Fine Structure of Helium. Ann. Phys. 1974, 82, 89.

[394] Hess, B. A. Applicability of the No-Pair Equation with Free-Particle Projection Operators to Atomic and Molecular Structure Calculations. Phys. Rev. A: At., Mol., Opt. Phys. 1985, 32, 756.

[395] Hess, B. A. Relativistic Electronic-Structure Calculations Employing a TwoComponent No-Pair Formalism with External-Field Projection Operators. Phys. Rev. A: At., Mol., Opt. Phys. 1986, 33, 3742.

[396] Jansen, G.; Hess, B. A. Revision of the Douglas-Kroll Transformation. Phys. Rev. A: At., Mol., Opt. Phys. 1989, 39, 6016.

[397] Peverati, R.; Truhlar, D. G. Communication: A Global Hybrid Generalized Gradient Approximation to the Exchange-Correlation Functional That Satisfies the SecondOrder Density-Gradient Constraint and Has Broad Applicability in Chemistry. $\mathcal{F}$. Chem. Phys. 2011, 135, 191102.

[398] Jorge, F. E.; Canal Neto, A.; Camiletti, G. G.; Machado, S. F. Contracted Gaussian Basis Sets for Douglas -Kroll -Hess Calculations: Estimating Scalar Relativistic Effects of Some Atomic and Molecular Properties. F. Chem. Phys. 2009, 130, 064108.

[399] Barros, C. L.; de Oliveira, P. J. P.; Jorge, F. E.; Canal Neto, A.; Campos, M. Gaussian Basis Set of Double Zeta Quality for Atoms Rb through Xe: Application in NonRelativistic and Relativistic Calculations of Atomic and Molecular Properties. Mol. Phys. 2010, 108, 1965.

[400] Campos, C. T.; Jorge, F. E. Triple Zeta Quality Basis Sets for Atoms Rb through Xe: Application in $\operatorname{CCSD}(\mathrm{T})$ Atomic and Molecular Property Calculations. Mol. Phys. 2013, 111, 167.

[401] Mitzel, N. W.; Smart, B. A.; Blake, A. J.; Robertson, H. E.; Rankin, D. W. H. Conformational Analysis of 1, 4-Disilabutane and 1,5-Disilapentane by Combined Application of Gas-Phase Electron Diffraction And ab Initio Calculations and the Crystal Structure of 1,5-Disilapentane at Low Temperatures. F. Phys. Chem. 1996, 100, 9339.

[402] Blake, A. J.; Brain, P. T.; McNab, H.; Miller, J.; Morrison, C. A.; Parsons, S.; Rankin, D. W. H.; Robertson, H. E.; Smart, B. A. Structure Analysis Restrained By ab Initio Calculations: The Molecular Structure of 2,5-Dichloropyrimidine in Gaseous and Crystalline Phases. F. Phys. Chem. 1996, 100, 12280. 
[403] Mitzel, N. W.; Rankin, D. W. H. SARACEN - Molecular Structures from Theory and Experiment: The Best of Both Worlds. Dalton Trans. 2003, 3650.

[404] Zhao, Y.; Truhlar, D. G. The M06 Suite of Density Functionals for Main Group Thermochemistry, Thermochemical Kinetics, Noncovalent Interactions, Excited States, and Transition Elements: Two New Functionals and Systematic Testing of Four M06-Class Functionals and 12 Other Function. Theor. Chem. Acc. 2008, 120, 215.

[405] Peverati, R.; Truhlar, D. G. Improving the Accuracy of Hybrid Meta-GGA Density Functionals by Range Separation. J. Phys. Chem. Lett. 2011, 2, 2810.

[406] Sipachev, V. A. Calculation of Shrinkage Corrections in Harmonic Approximation. f. Mol. Struct.: THEOCHEM 1985, 121, 143.

[407] Sipachev, V. A. Local Centrifugal Distortions Caused by Internal Motions of Molecules. F. Mol. Struct. 2001, 567-568, 67.

[408] Rankine, C. D.; Nunes, J. P. F.; Feixas, T. W. B. L.; Young, S.; Wann, D. A. Structure of 4-(Dimethylamino)Benzonitrile Using Gas Electron Diffraction: A New Lease of Life for the Only Gas Electron Diffractometer in the U.K. F. Phys. Chem. A 2018, $122,5656$.

[409] Nunes, J. Software Package: xtract. 2017.

[410] Hinchley, S. L.; Robertson, H. E.; Borisenko, K. B.; Turner, A. R.; Johnston, B. F.; Rankin, D. W. H.; Ahmadian, M.; Jones, J. N.; Cowley, A. H. The Molecular Structure of Tetra-tert-Butyldiphosphine: An Extremely Distorted, Sterically Crowded Molecule. Dalton Trans. 2004, 2469.

[411] Prince, E., Ed. International Tables for Crystallography; International Union of Crystallography, 2006. 